LA-14324-T

Thesis

Approved for public release;

distribution is unlimited.

Numerical and Experimental Investigations

of a Rotating Heat Pipe 
Funding provided by the Department of Energy, Office of Energy Efficiency and Renewable Energy

This thesis was accepted by the Department of Mechanical Engineering, University of New Mexico, Albuquerque, New Mexico, in partial fulfillment of the requirements for the degree of Doctor of Philosophy. The text and illustrations are the independent work of the author, and only the front matter has been edited by the IRM-CAS Writing and Editing staff to conform with Department of Energy and Los Alamos National Laboratory publication policies.

Los Alamos National Laboratory, an affirmative action/ equal opportunity employer, is operated by Los Alamos National Security, LLC, for the National Nuclear Security Administration of the U.S. Department of Energy under contract DE-AC52-06NA25396.

This report was prepared as an account of work sponsored by an agency of the U.S. Government. Neither Los Alamos National Security, LLC, the U.S. Government nor any agency thereof, nor any of their employees make any warranty, express or implied, or assume any legal liability or responsibility for the accuracy, completeness, or usefulness of any information, apparatus, product, or process disclosed, or represent that its use would not infringe privately owned rights. Reference herein to any specific commercial product, process, or service by trade name, trademark, manufacturer, or otherwise does not necessarily constitute or imply its endorsement, recommendation, or favoring by Los Alamos National Security, LLC, the U.S. Government, or any agency thereof. The views and opinions of authors expressed herein do not necessarily state or reflect those of Los Alamos National Security, LLC, the U.S. Government, or any agency thereof. Los Alamos National Laboratory strongly supports academic freedom and a researcher's right to publish; as an institution, however, the Laboratory does not endorse the viewpoint of a publication or guarantee its technical correctness. 
LA-14324-T

Thesis

Issued: March 2007

Numerical and Experimental Investigations

of a Rotating Heat Pipe

Todd A. Jankowski 


\section{NUMERICAL AND EXPERIMENTAL INVESTIGATIONS OF}

A ROTATING HEAT PIPE

\section{BY}

\section{TODD A. JANKOWSKI}

B. S., Mechanical Engineering, Marquette University, 1999

M. S., Mechanical Engineering, Marquette University, 2001

\section{DISSERTATION}

Submitted in Partial Fulfillment of the

Requirements for the Degree of

Doctor of Philosophy

Engineering

The University of New Mexico

Albuquerque, New Mexico

May, 2007 


\section{ACKNOWLEDGMENTS}

I gratefully acknowledge the help and guidance of my academic advisor, Prof. Arsalan Razani. He offered his direction, support, and encouragement throughout the completion of this work. I also thank the other members of my dissertation committee, Prof. Evangelos A. Coutsias, Prof. James R. Leith, Dr. F. Coyne Prenger, and Prof. C. Randall Truman. The comments and suggestions given during their reviews of the dissertation are greatly appreciated.

This work would not have been possible without the contributions of all those involved at the Los Alamos National Laboratory. Thank you to Mr. Jim Stewart and Mr. Dallas Hill for their help with the design and construction of the experimental apparatus. I thank Dr. Eric Schmierer and Dr. Joe Waynert for spending much of their time discussing various aspects of the project. I owe a great deal to my current mentor at Los Alamos, Dr. Coyne Prenger. He taught me everything that I claim to know, and showed me that I have much more to learn, about heat pipes.

Finally, I wish to acknowledge the DOE Office of Energy Efficiency and Renewable Energy, and support from Dr. Dean Peterson of the Superconductivity Technology Center at Los Alamos, for funding this project. 
NUMERICAL AND EXPERIMENTAL INVESTIGATIONS OF A ROTATING HEAT PIPE

\title{
BY
}

TODD A. JANKOWSKI

\author{
ABSTRACT OF DISSERTATION \\ Submitted in Partial Fulfillment of the \\ Requirements for the Degree of \\ Doctor of Philosophy \\ Engineering \\ The University of New Mexico \\ Albuquerque, New Mexico
}

May, 2007 


\title{
NUMERICAL AND EXPERIMENTAL INVESTIGATIONS OF A ROTATING \\ HEAT PIPE
}

By

\section{Todd A. Jankowski}

B. S., Mechanical Engineering, Marquette University, 1999

M. S., Mechanical Engineering, Marquette University, 2001

Ph. D., Engineering, The University of New Mexico, 2007

\begin{abstract}
Rotating and revolving heat pipes have been used in a variety of applications including heat pipe heat exchangers, cooling of rotating electrical machines, and heat removal in high speed cutting operations. The use of heat pipes in rotating environments has prompted many analytical, numerical, and experimental investigations of the heat transfer characteristics of these devices. Past investigations, however, have been restricted to the study of straight heat pipes.

In this work, a curved rotating heat pipe is studied numerically and experimentally. In certain types of rotating machines, heat generating components, which must be cooled during normal operation, are located at some radial distance from the axis of rotation. The bent heat pipe studied here is shown to have advantages when compared to the conventional straight heat pipes in these off-axis cooling scenarios.

The heat pipe studied here is built so that both the condenser and evaporator sections are parallel to the axis of rotation. The condenser section is concentric with the axis of
\end{abstract}


rotation while the evaporator section can be placed in contact with off-axis heat sources in the rotating machine. The geometry is achieved by incorporating an S-shaped curve between the on-axis rotating condenser section and the off-axis revolving evaporator section. Furthermore, the heat pipe uses an annular gap wick structure. Incorporating an annular gap wick structure into the heat pipe allows for operation in a non-rotating environment.

A numerical model of this rotating heat pipe is developed. The analysis is based on a two-dimensional finite-difference model of the liquid flow coupled to a one-dimensional model of the vapor flow. Although the numerical model incorporates many significant aspects of the fluid flow, the flow in the actual heat pipe is expected to be threedimensional. The rotating heat pipe with the S-shaped curve is also studied experimentally to determine how well the numerical model captures the key aspects of the fluid flow and heat transfer. 


\section{TABLE OF CONTENTS}

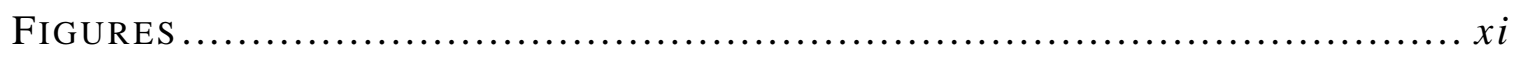

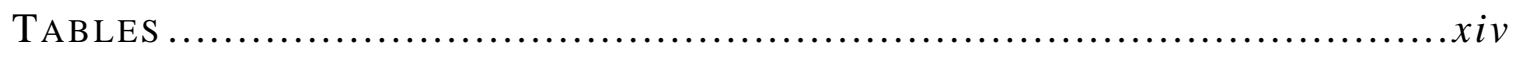

NOMENCLATURE.........................................................

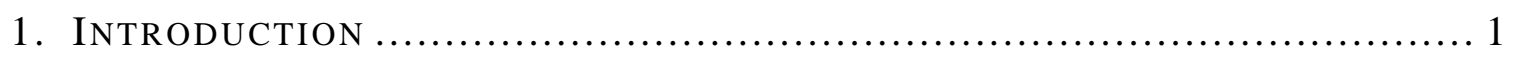

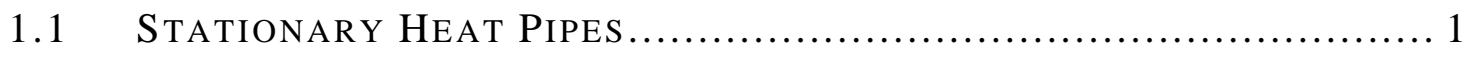

1.2 Rotating and Revolving Heat Pipes $\ldots \ldots \ldots \ldots \ldots \ldots \ldots \ldots \ldots \ldots \ldots . \ldots . \ldots \ldots$

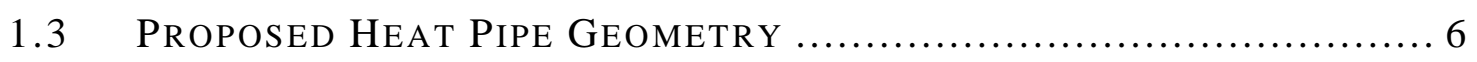

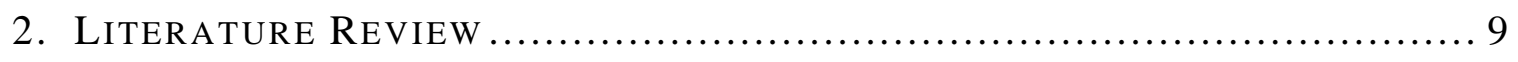

2.1 Modeling of Stationary Heat Pipes ............................. 9

2.2 Modeling of Rotating and Revolving Heat Pipes.............. 11

2.2.1 Liquid Flow Modeling in Rotating Heat Pipes............. 11

2.2.2 Complete Models of Rotating Heat Pipes ................... 13

2.2.3 Revolving and Radially Rotating Heat Pipes............. 14

2.3 Experiments With Rotating and Revolving Heat Pipes ..... 15

2.3.1 Liquid Flow Regimes in Rotating Heat Pipes .............. 15

2.3.2 High Speed Rotating Heat Pipes ............................. 17

2.3.3 Radially Rotating Heat Pipes.............................. 19

2.3.4 Revolving Heat Pipes ................................... 20

2.4 Applications of Rotating and Revolving Heat Pipes......... 21

3. Rotating Heat Pipe Model ......................................... 23

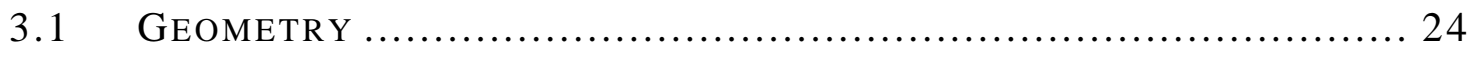

3.2 Assumptions ................................................... 25 
3.3 Mathematical Model and Numerical Analysis ................ 25

3.3.1 Governing Equations and Boundary Conditions ......... 25

3.3.2 CoORdinate Transformations And Dimensionless

PARAMETERS ............................................................ 28

3.3.3 Natural Convection in the Evaporator.................... 33

3.3.4 VAPOR Flow MOdel ...................................... 35

3.3.5 Numerical SOLUtion ........................................... 38

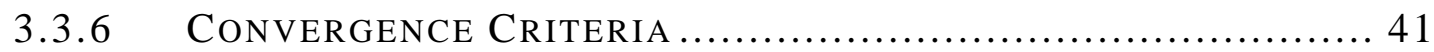

3.4 VERIFICATION ANd VAlidation................................. 42

3.4.1 Numerical Verification ................................ 43

3.4 .2 VALidATION .................................................... 46

3.5 Results for the Proposed Heat Pipe .......................... 49

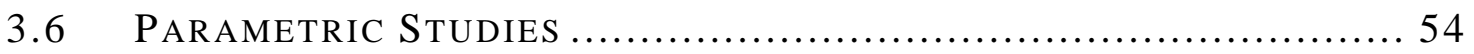

3.6.1 Condenser Heat Transfer ................................. 54

3.6.2 Fixed Geometry and Fluid Charge ........................ 57

3.6.3 The Effect of Fluid Charge.............................. 58

3.6.4 InCREASING THE LENGTH OF THE EVAPORATOR ................ 60

3.6.5 InCREASing the Distance from the Axis of Rotation .... 61

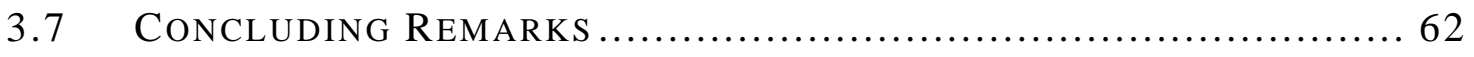

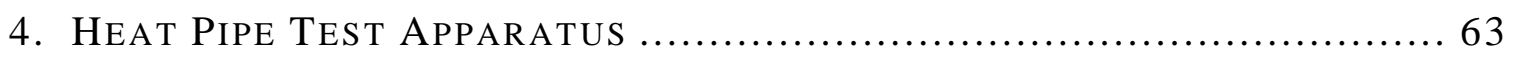

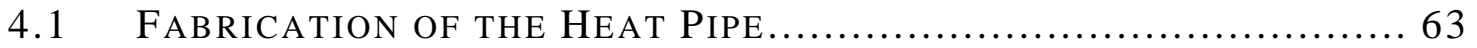

4.2 Rotating Test Apparatus ...................................... 66

4.3 Supporting Analyses............................................... 71 


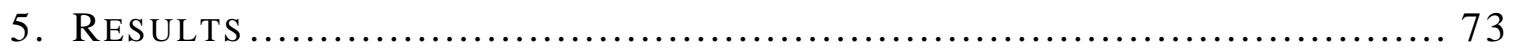

5.1 Stationary Bench-TOP TESTS ....................................... 73

5.2 Tests in the Rotating Apparatus .................................. 75

5.2.1 REPORTED UnCERTAINTIES ..................................... 75

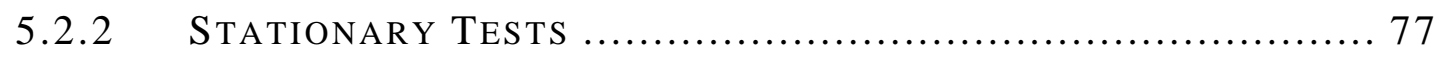

5.2.3 LOW SPEED Dry-OUt TeStS ...................................... 77

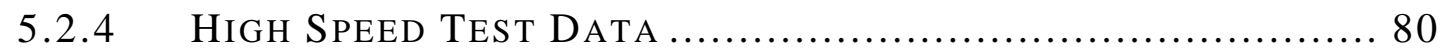

5.3 Comparisons to the Rotating Heat Pipe Model ................. 83

5.3.1 Mixed Convection and Turbulence in the Liquid ......... 85

5.3.2 Three-Dimensional Pool Flow .................................. 86

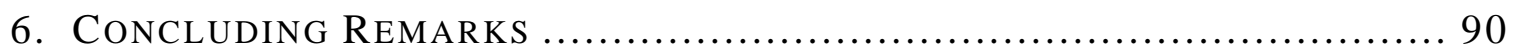

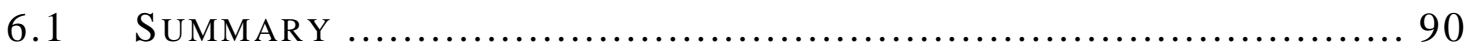

6.2 Suggestions for FutUre Work ................................... 93

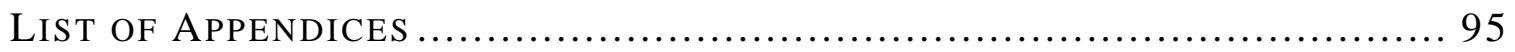

APPENDIX A: SIMPLER .................................................... 96

Appendix B: Heat Transfer In An Evaporating Liquid Layer ........102

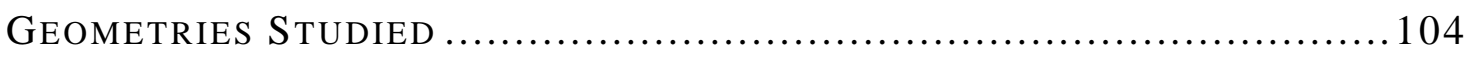

Mathematical Model And Numerical Analysis .........................104

Governing Equations And Boundary Conditions .....................104

Coordinate Transformations and Dimensionless Parameters.106

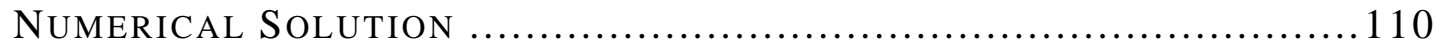

Convergence Criteria, Observed Convergence Rates, and Error

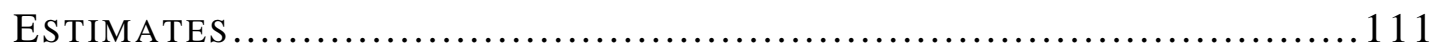




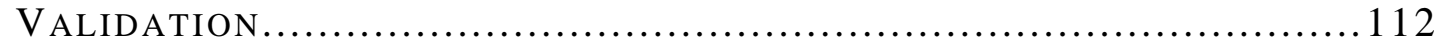

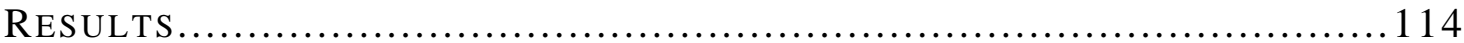

Streamlines, Isotherms, and Surface Deformation................114

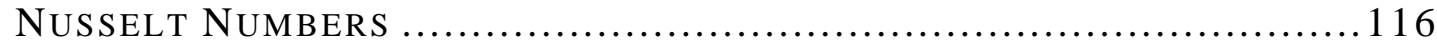

Appendix C: Experimental Uncertainty Analysis ........................ 121

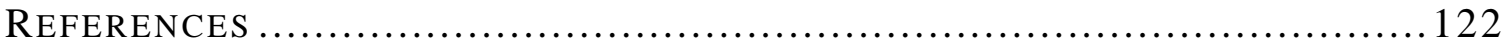




\section{FIGURES}

Figure 1.1. A cut-away view of a typical heat pipe. ....................................................2

Figure 1.2. Some wick structures typically used in heat pipes. ......................................2

Figure 1.3. Pressure vs. length for a horizontal non-rotating heat pipe. ..........................3

Figure 1.4. The cylindrical rotating heat pipe.........................................................5

Figure 1.5. The proposed heat pipe geometry..........................................................

Figure 1.6. Cross-sectional view of the rotating heat pipe. ........................................

Figure 3.1. An illustration of the liquid layer and parameters used in the model.............24

Figure 3.2. Pressure distributions from present model and HTPIPE for a stationary heat

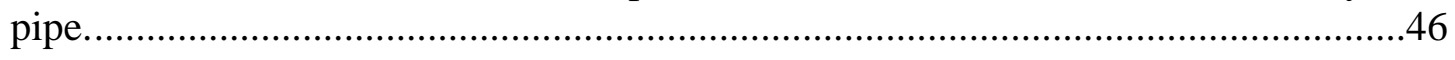

Figure 3.3. Model results for an axially rotating heat pipe compared to the results of the

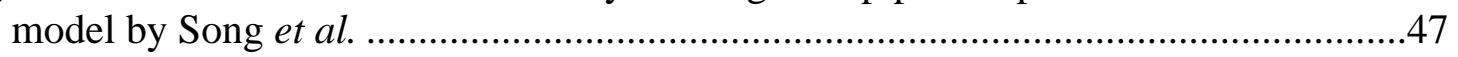

Figure 3.4. Liquid layer thickness in the tapered rotating condenser section..................48

Figure 3.5. Temperature difference across the condenser film required to transfer heat in the rotating tapered condenser section..............................................................48

Figure 3.6. The rotating heat pipe modeled here. All dimensions are in centimeters. ....49

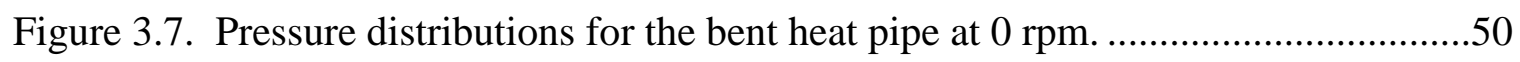

Figure 3.8. Curvature supported by the wick for the bent heat pipe at $0 \mathrm{rpm}$.................51

Figure 3.9. Pressure distributions for the bent heat pipe at $45 \mathrm{rpm}$...............................52

Figure 3.10. Curvature supported by the wick for the bent heat pipe at $45 \mathrm{rpm}$..............52

Figure 3.11. Pressure distributions for the bent heat pipe at $200 \mathrm{rpm}$...........................53

Figure 3.12. Curvature supported by the wick for the bent heat pipe at $200 \mathrm{rpm}$............53

Figure 3.13. Liquid layer thickness for the bent heat pipe at $200 \mathrm{rpm} . . . \ldots \ldots \ldots \ldots \ldots \ldots \ldots . . . . . . .54$

Figure 3.14. Condenser film temperature difference for the baseline heat pipe..............55

Figure 3.15. Temperature difference across the condenser film for various annular gap

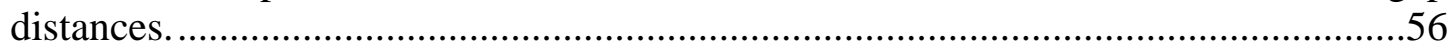


Figure 3.16. Reynolds and Péclet numbers in the liquid film for the baseline rotating heat pipe.

Figure 3.17. Temperature difference across the evaporator film for various heat loads

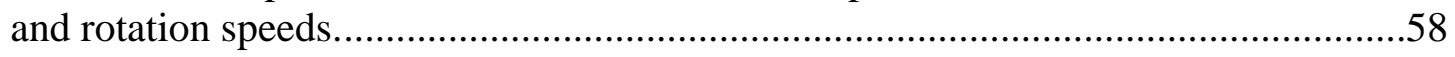

Figure 3.18. Evaporator film temperature difference as the fill mass is varied................59

Figure 3.19. The effect of evaporator length on temperature difference across the evaporator film for a constant liquid layer thickness. 60

Figure 3.20. Temperature difference over the adiabatic section as the distance from the off-axis evaporator to the on-axis condenser is increased.

Figure 4.1. The bias wrapped wick. 64

Figure 4.2. The rotating test apparatus. (a) Top and (b) side views are given................67

Figure 4.3. G-10 support structure and stainless steel shield. .68

Figure 4.4. Data from calibration tests of the thermocouple measurements in (a) icewater and (b) boiling water. .70

Figure 4.5. The test apparatus during assembly. In (a) a view of the completed assembly in the frame is shown. Figures (b), (c), and (d) show the condenser end assembly. A close-up of the large bearing with the condenser sections extending out is in (b), the condensers with the copper cover blocks are shown in (c), and in (d) the water jacket has been mounted at the condenser end. Figure (e) shows the evaporator sections and G-10 supports and (f) shows the assembly with the stainless steel shield. .71

Figure 5.1. The bench-top test stand with the straight heat pipe. The overall set-up is shown in (a), and the copper chill-block on the condenser is shown in (b).................74

Figure 5.2. Example data file for the stationary test with the straight heat pipe with the condenser set to $20 \mathrm{C}$. .75

Figure 5.3. Bench-top test results (a) before bending and (b) after bending. .76

Figure 5.4. Estimated heat loss through the G-10 supports and insulation for the heat pipe installed in the rotating apparatus. .78

Figure 5.5. Stationary tests with the heat pipes installed in the rotating test apparatus....78

Figure 5.6. Low-speed dry-out tests.

Figure 5.7. Example data file for a rotating test with a $150 \mathrm{~W}$ heat load and a condenser temperature of $25 \mathrm{C}$. 
Figure 5.8. Measured temperature difference across the evaporator liquid film for the rotating tests. All uncertainties are the same as the $38 \mathrm{~W}$ case.....

Figure 5.9. Conductance across the liquid film in the evaporator section of the rotating heat pipe. The $74 \mathrm{~W}$ case gave the largest (dashed) error bar and the $198 \mathrm{~W}$ case gave the smallest (solid) error bar when calculating the conductance.

Figure 5.10. Nusselt number for heat transfer across the evaporator film in the rotating heat pipe.

Figure 5.11. Comparing experimental results to the model using the convection correlation of Jankowski et al. .

Figure 5.12. Comparing experimental results to the model using the convection correlation of Körner.

Figure 5.13. Comparing heat transfer coefficients in the evaporator. The dashed error bars are for the $74 \mathrm{~W}$ data and the $198 \mathrm{~W}$ error bars are solid. .88

Figure A.1. The finite-difference stencil used for integrating the equations. .96

Figure B.1. Geometries studied. The fixed upper plate case (Rayleigh-Bénard) is shown in (a), while (b) is an illustration of the free-surface case. .105

Figure B.2. Streamlines and isotherms for natural convection in a 4 x 1 system for the fixed upper plate case [isotherms in (a) and streamlines in (b)] and for the case with free-surface evaporation [isotherms in (c) and streamlines in (d)]. Both cases simulate convection in water with $R a=10^{4}$. .115

Figure B.3. Surface profile for the free-surface evaporation case. Results are for convection in water with $R a=10^{4}$. .116

Figure B.4. Comparing convection in a $4 \times 1$ box for the fixed upper surface, freesurface, and free-surface with evaporation cases. In (a) the working fluid is water, and in (b) the fluid is methanol. .117

Figure B.5. Comparing the free-surface case with evaporation to the free-surface case without evaporation with a Jakob number of 0.2 in (a) water and (b) methanol.......119

Figure B.6. Calculated Nusselt number compared to the correlation in Eq. (B.32)......120 


\section{TABLES}

Table 3.1. Dimensionless parameters in the numerical model. .34

Table 3.2. Results of the grid convergence study for (a) the non-rotating heat pipe, (b) the cylindrical rotating heat pipe, and (c) the tapered condenser section.

Table B.1. Grid convergence studies for (a) the Rayleigh-Bénard problem and (b) the free-surface problem with evaporation. Both are carried out in a $4 \times 1$ box............113

Table B.2. Comparison between a benchmark solution for natural convection in a square side-heated cavity and the present numerical solution. .114 


\section{NOMENCLATURE}

English:

$a \quad$ velocity profile correction factor

$A_{c}$ surface area of condenser

$A_{e}$ surface area of evaporator

$C^{\phi}$ pseudo-diffusion term

$\bar{C}^{\bar{\phi}}$ dimensionless pseudo-diffusion term

Ca Capillary number, $\rho \mathrm{v}_{\text {char }}^{2} L / \sigma$

$C_{p}$ specific heat

$d$ distance from axis of rotation

$\bar{d}$ dimensionless distance from axis of rotation, $d / R_{0}$

$d_{a}$ thickness of rotating annulus

$f \quad$ Darcy friction factor

Fr Froude number, $\omega^{2} d / g$

$g$ downward acceleration

$\bar{g}$ dimensionless acceleration, $g L / \mathrm{v}_{\text {char }}^{2}$

$H$ curvature of free-surface

$h_{e}$ heat transfer coefficient in the evaporator section

$h_{f g}$ latent heat

$H_{\max }$ maximum curvature of free-surface

Gr Grashof number, $R a / P r$
Ja Jakob number, $C_{p}\left(T_{w}-T_{s}\right) / h_{f g}$

$k \quad$ thermal conductivity

$L \quad$ length in horizontal direction

$L_{a}$ length of adiabatic section

$L_{c}$ length of condenser section

$L_{e}$ length of evaporator section

$L_{t o t}$ total length of heat pipe

$\dot{m}$ mass flow rate

$\bar{m}$ dimensionless flow rate, $\dot{m} /\left(\rho \mathrm{v}_{c h} R_{0}^{2}\right)$

$n$ exponent in $\mathrm{Nu} \sim R a^{n}$ relationship

n unit vector normal to free-surface

$\mathbf{n}_{\mathbf{w}}$ unit vector normal to heat pipe wall

$\mathrm{Nu}$ Nusselt number

Oh Ohnesorge number, $\mu / \sqrt{\rho L \sigma}$

p pressure

$P$ modified pressure, $p+\rho g y$

Pe Péclet number, $\rho C_{p} \mathrm{v}_{\text {char }} L / k$

Pr Prandtl number, $\mu C_{p} / k$

Q heat transfer rate

$q^{\prime \prime}$ heat flux

$\vec{q}^{\prime \prime}$ dimensionless heat flux 
$r$ radial coordinate

$R$ radius of heat pipe container

$R^{\prime}, R^{\prime \prime}$ derivatives with respect to $x$

$\bar{R} \quad$ dimensionless radius, $R / R_{0}$

$\bar{R}^{\prime}, \bar{R}^{\prime \prime}$ derivatives with respect to $\chi$

$R_{0}$ radius at condenser end-cap

$r_{\text {eff }}$ effective pore radius of wick

$r_{m} \quad$ mean radius of rotating annulus

$R_{n}$ residual of normal stress condition

$\mathrm{Ra}$ Rayleigh number, $\rho^{2} C_{p} \delta^{3} \beta g\left(T_{w}-T_{s}\right) /(\mu k)$ or $\rho^{2} C_{p} \delta^{3} \beta \omega^{2} d\left(T_{w}-T_{s}\right) /(\mu k)$

Re Reynolds number, $\rho \mathrm{v}_{\text {char }} L / \mu$

$\mathrm{Re}_{r}$ radial Reynolds number

$S^{\phi}$ source terms

$\bar{S}^{\bar{\phi}}$ dimensionless source terms

$T$ temperature

$T_{c h}$ characteristic temperature

$T_{s} \quad$ saturation temperature

u dimensionless velocity, $\mathbf{v} / \mathbf{v}_{c h}$

v velocity

$V \quad$ volume of liquid in heat pipe

$\mathrm{V}_{c h}$ characteristic velocity $v_{f g}$ volume change during vaporization

$x$ horizontal/axial coordinate

y vertical coordinate

z dimensionless horizontal coordinate

Greek:

$\alpha$ angle between axis of rotation and raxis

$\beta$ volume expansion coefficient

$\bar{\beta}$ dimensionless volume expansion coefficient, $\beta\left(T_{w}-T_{s}\right)$

$\gamma \quad$ apparent order of numerical method

$\Gamma \quad$ diffusion coefficient

$\bar{\Gamma}$ dimensionless diffusion coefficient

$\delta \quad$ liquid-layer thickness

$\delta^{\prime}, \delta^{\prime \prime}$ derivatives with respect to $x$

$\bar{\delta}$ dimensionless layer thickness

$\bar{\delta}^{\prime}, \bar{\delta}^{\prime \prime}$ derivatives with respect to $\chi$

$\delta_{0} \quad$ layer thickness at condenser end-cap

$\varepsilon($ ) uncertainty in measured or calculated value

$\eta$ dimensionless vertical coordinate

$\theta$ circumferential coordinate

$\kappa$ relaxation factor

$\Lambda$ dimensionless temperature

$\mu \quad$ viscosity 
$\xi$ dimensionless radial coordinate

$\Pi$ dimensionless pressure

$\rho$ density

$\sigma$ surface tension

$\tau$ shear stress

$\phi \quad$ general dependent variable

$\bar{\phi}$ dimensionless general dependent variable

$\chi$ dimensionless axial coordinate

$\Psi$ general result in uncertainty propagation analysis

$\omega$ rotation speed

$\Omega$ dimensionless rotation speed
Subscripts:

$F$ forced convection

$N$ natural convection

$r$ r-direction

$v \quad$ vapor

$x \quad \mathrm{X}$-direction

y y-direction

Z $\quad$ Z-direction

$\eta \quad \eta$-direction

$\theta \quad \theta$-direction

$\chi \quad \chi$-direction 


\section{INTRODUCTION}

This chapter serves as an introduction to the theory and operating principles of heat pipes. The introduction is presented in three parts. First, heat pipes operating in a stationary or non-rotating mode are introduced and explained, followed by an introduction to rotating and revolving heat pipes. A rotating heat pipe that is being proposed for use in off-axis cooling of rotating machinery is then presented.

\subsection{Stationary HEAT PiPES}

Heat pipes are devices that rely on the evaporation, condensation, and circulation of a working fluid to transfer heat between a heat source and a heat sink. A typical heat pipe is illustrated in Fig. 1.1. The heat pipe shown is comprised of a cylindrical tube that is sealed at both ends. The inner wall of the cylindrical tube is lined with a porous wick structure. Some typical wick structures are illustrated in Fig. 1.2. The heat pipe is filled with a working fluid. The amount of fluid added to the heat pipe, or the fluid charge, is determined so that the working fluid will remain a saturated liquid-vapor mixture over the entire operating temperature range of the heat pipe. In a typical heat pipe, the liquid phase occupies the wick structure, and when the evaporator end of the heat pipe is heated, a portion of the liquid phase vaporizes at the liquid-vapor interface. A difference in pressure, in the vapor phase, created by a temperature difference between the evaporator and condenser ends of the heat pipe is used to drive the vapor flow through the adiabatic section and into the condenser. In the condenser section, which is in contact with an external heat sink, a portion of the vapor condenses at the liquid-vapor interface as heat is rejected to the heat sink. A pressure difference between the condenser and evaporator sections, in the liquid phase, is then used to drive the liquid flow from the condenser 


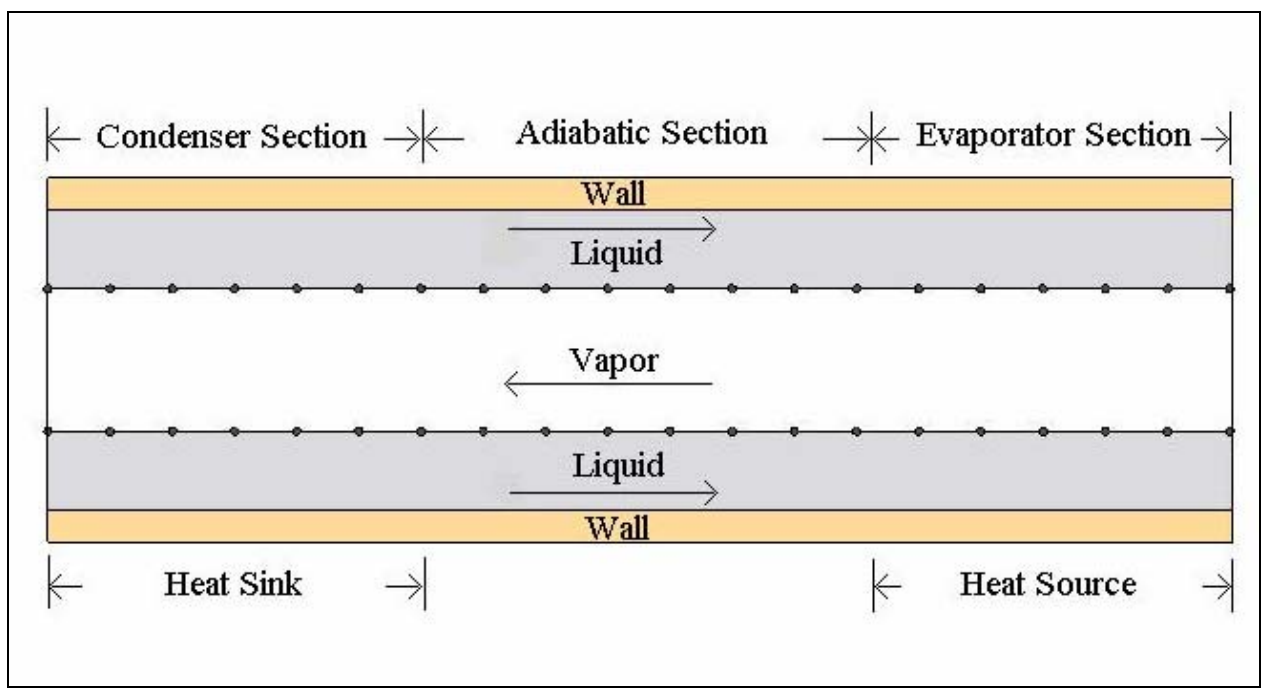

Figure 1.1. A cut-away view of a typical heat pipe.

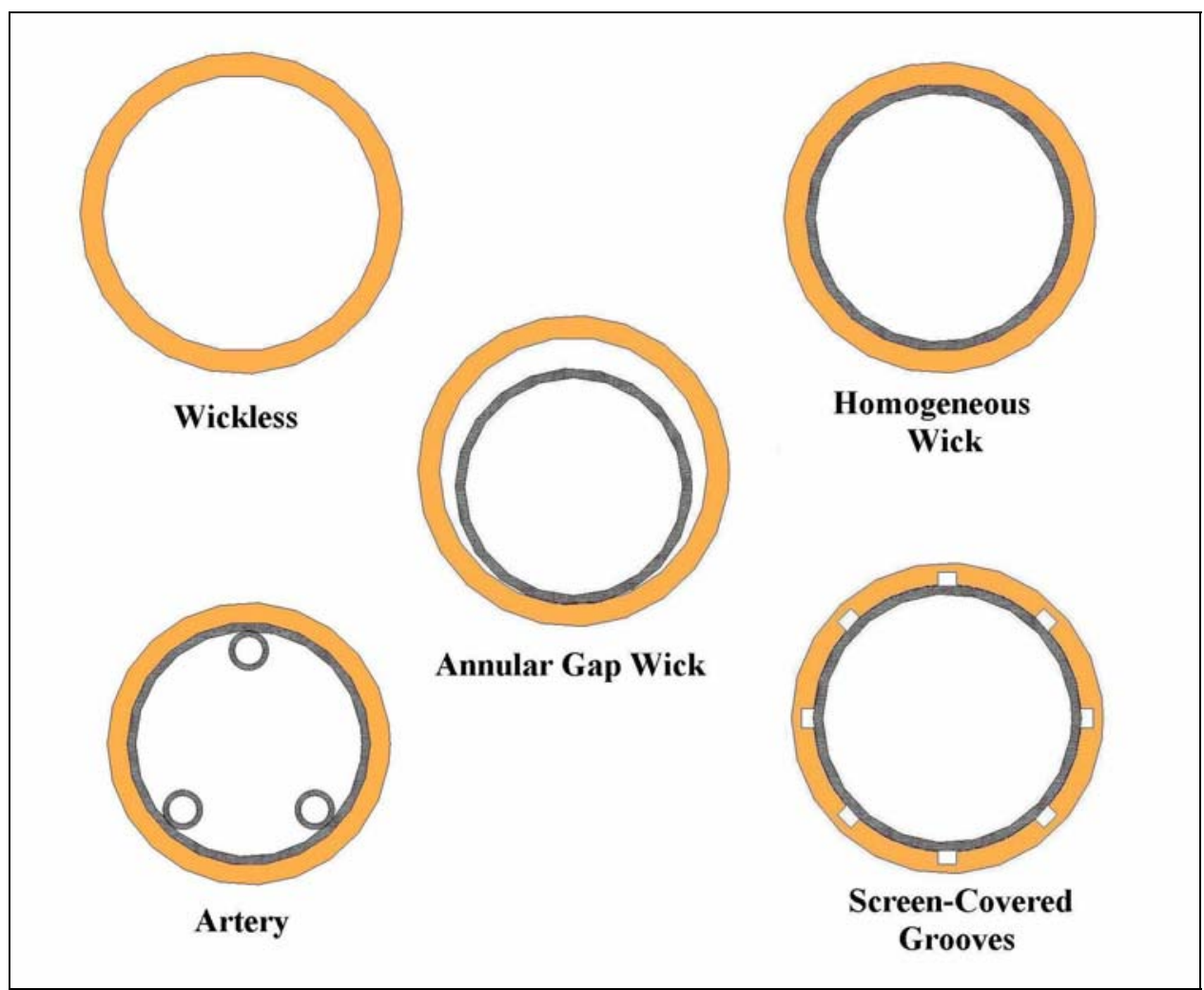

Figure 1.2. Some wick structures typically used in heat pipes.

toward the evaporator.

A typical pressure versus length curve, for a horizontal non-rotating heat pipe, is shown in Fig. 1.3. The figure shows that a wet-point (a point where the pressure of the liquid is equal to the pressure of the vapor) is typically located somewhere in the 
condenser section of the heat pipe, and that the maximum pressure difference between the liquid and vapor phases must supported at the end of the evaporator section. Surface tension in the porous wick is used to support this pressure difference at the liquid-vapor interface. The pressure difference required of the wick, as well as the shape of the pressure curves shown in Fig. 1.3, will be influenced by the presence of body forces (gravity) acting on the working fluid. This observation suggests that the performance of a heat pipe is influenced by physical orientation.

The four most common limitations on the maximum possible heat transfer rate encountered in the operation of heat pipes are the capillary limit, boiling limit, entrainment limit, and sonic limit.

The capillary limit can be understood by considering Fig. 1.3. As the heat transfer rate is increased, resulting in increased mass flow rates in the liquid and vapor phases, the pressure drops in both the liquid and vapor phases increase. Increasing the pressure drops in the heat pipe causes the maximum pressure difference between the liquid and

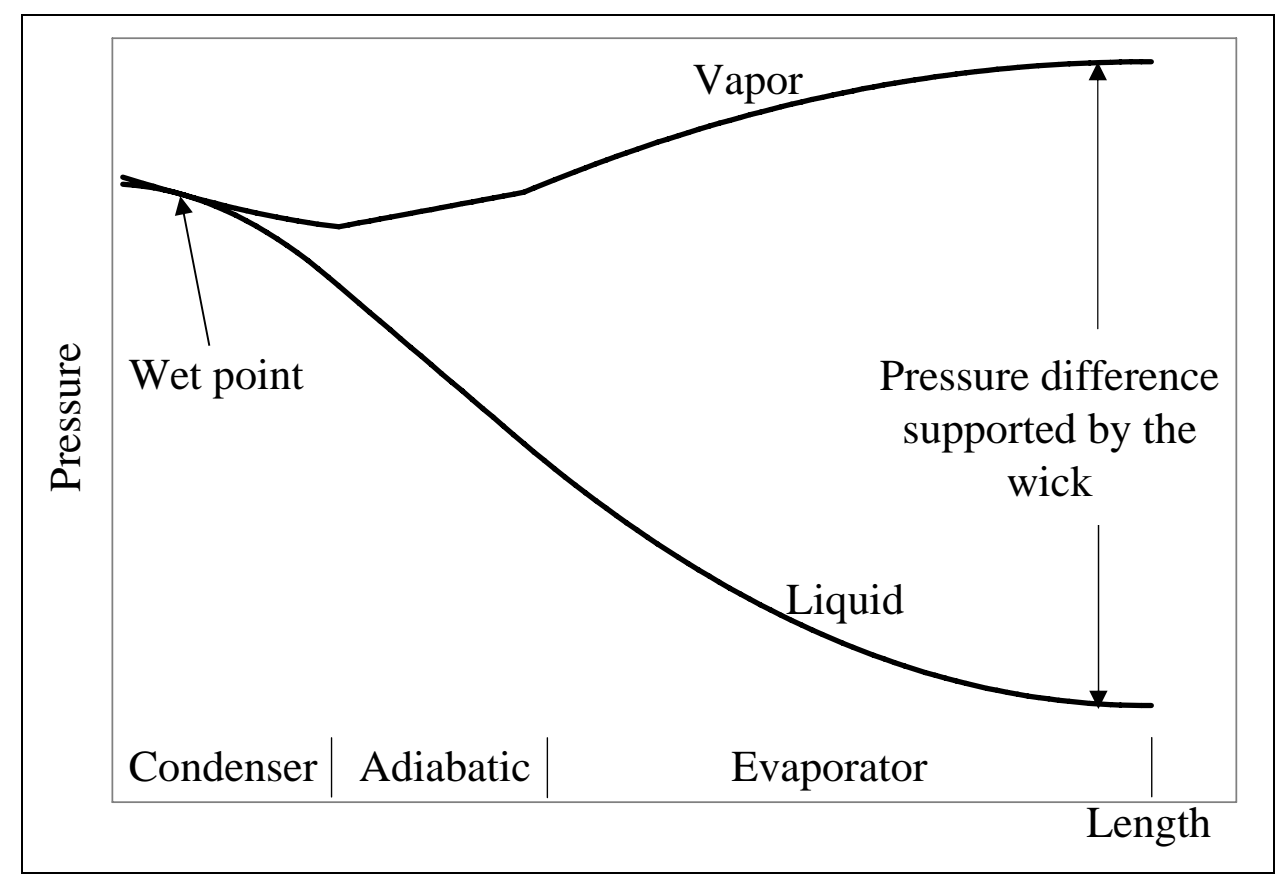

Figure 1.3. Pressure vs. length for a horizontal non-rotating heat pipe. 
vapor phases to increase (typically reaching a maximum at the end of the evaporator). If this maximum pressure difference exceeds the maximum capillary pressure of the wick, the driving force for liquid flow will be insufficient, and a so-called dry-out of the evaporator will occur.

During normal operation of a heat pipe, the primary mechanism for heat transfer across the liquid layer in the evaporator section is by conduction. As the heat flux to the evaporator section is increased, nucleate boiling can be induced in the wick structure. The vapor bubbles produced at the inner wall of the heat pipe can become trapped in the wick structure, again causing a dry-out. Postponing the onset of the boiling limit can be achieved by increasing the effective thermal conductivity of the wick structure or reducing the size of nucleation sites on the inner wall of the heat pipe.

The entrainment limit is a result of the interaction between the counter-current liquid and vapor flows. The relative velocity of the liquid and vapor phases increases as the heat transfer rate is increased. The resulting shear stress at the liquid-vapor interface can cause the liquid to be swept into the condenser section by the vapor flow. The entrainment of liquid into the vapor flow prevents liquid from reaching the evaporator section, causing a dry-out.

The sonic limit of a heat pipe is reached when the heat transfer rate is high enough to cause the velocity of the vapor flow to reach the speed of sound. Sonic flow in the constant area vapor space results in a mass flow, and consequently heat transfer, limitation. Unlike the other limitations, this is a stable condition and does not result in an evaporator dry-out. 


\subsection{Rotating And Revolving Heat Pipes}

In many applications, the need arises for use of a heat pipe in a rotating environment. The most common applications of rotating and revolving heat pipes involve wickless heat pipes (sometimes referred to as thermosyphons) that use the centrifugal force for liquid return to the evaporator. The rotating heat pipe, as shown in Fig. 1.4, is a heat pipe rotating about its own longitudinal axis. Due to the centrifugal force acting on the working fluid, a variable thickness liquid film is formed along the internal wall of the heat pipe, and the vapor occupies the central core. For the cylindrical rotating heat pipe, the liquid layer is thickest in the condenser section and becomes gradually thinner as the liquid moves toward the evaporator. This variable thickness film, and the resulting hydrostatic head caused by the rotation, generates a pressure difference in the liquid that pumps liquid from the condenser to the evaporator.

The heat addition to and heat rejection from the rotating heat pipe are accommodated internally by a phase change of the working fluid at the liquid-vapor interface. Depending on the working fluid, significant thermal resistances in the rotating heat pipe can be attributed to heat transfer through the liquid layer lining the inner wall of the

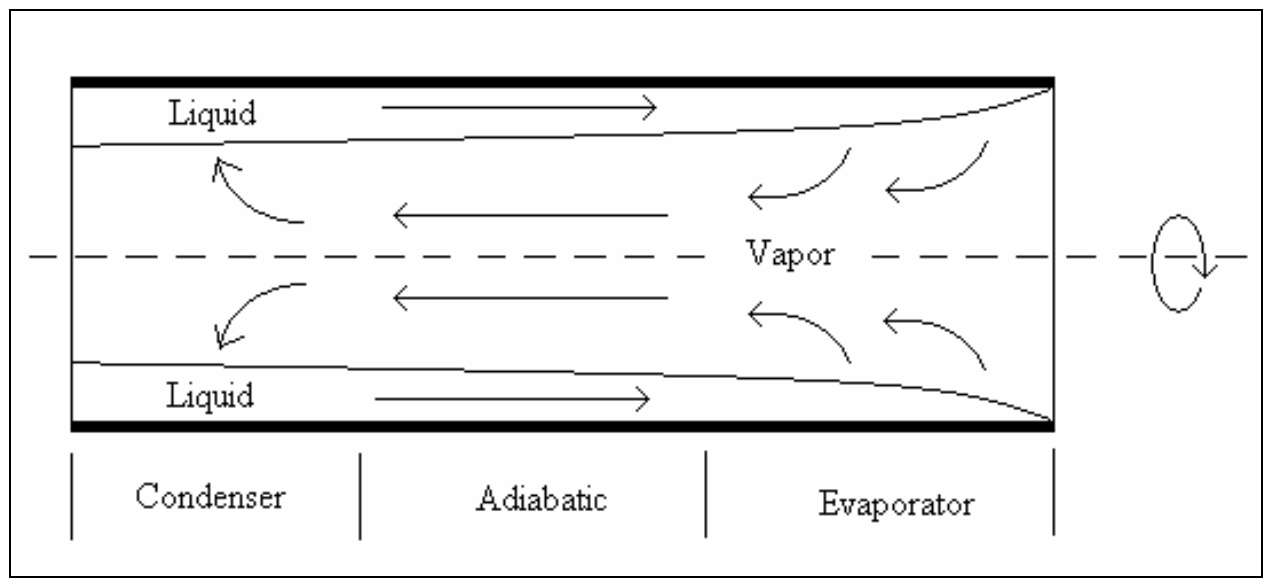

Figure 1.4. The cylindrical rotating heat pipe. 
evaporator and condenser sections. Characterizing and predicting the performance of rotating heat pipes requires a determination of these thermal resistances.

Many rotating heat pipes have used condensers that are tapered from small diameter at the condenser end-cap to larger diameter at the entrance to the adiabatic section. Tapered designs allow a component of the centrifugal force to act directly in the liquid flow direction. When compared to the cylindrical geometry, tapered heat pipes typically operate with thinner liquid films in the condenser, which reduces the thermal resistance.

In applications where heat sources are located at some distance from the axis of rotation, designs have been suggested using radially rotating and revolving heat pipes. In the radially rotating heat pipe, the longitudinal axis of the heat pipe is perpendicular to the axis of rotation, with the evaporator section farthest away from the axis and the condenser section close to the axis. This orientation allows for the centrifugal force to be used for liquid return to the evaporator. The longitudinal axis of the revolving heat pipe is parallel to, but displaced from, the axis of rotation, so that the evaporator can be placed in direct contact with off-axis heat sources.

\subsection{Proposed Heat Pipe Geometry}

A unique rotating heat pipe that combines many of the features of rotating, radially rotating, and revolving heat pipes is studied here. The proposed heat pipe geometry is shown in Fig. 1.5. The heat pipe consists of an on-axis rotating condenser section, an Sshaped transition section, and an off-axis revolving evaporator section.

A cross-sectional view of the heat pipe is shown in Fig 1.6. The heat pipe utilizes an annular gap composite wick structure. The presence of the annular gap wick structure 


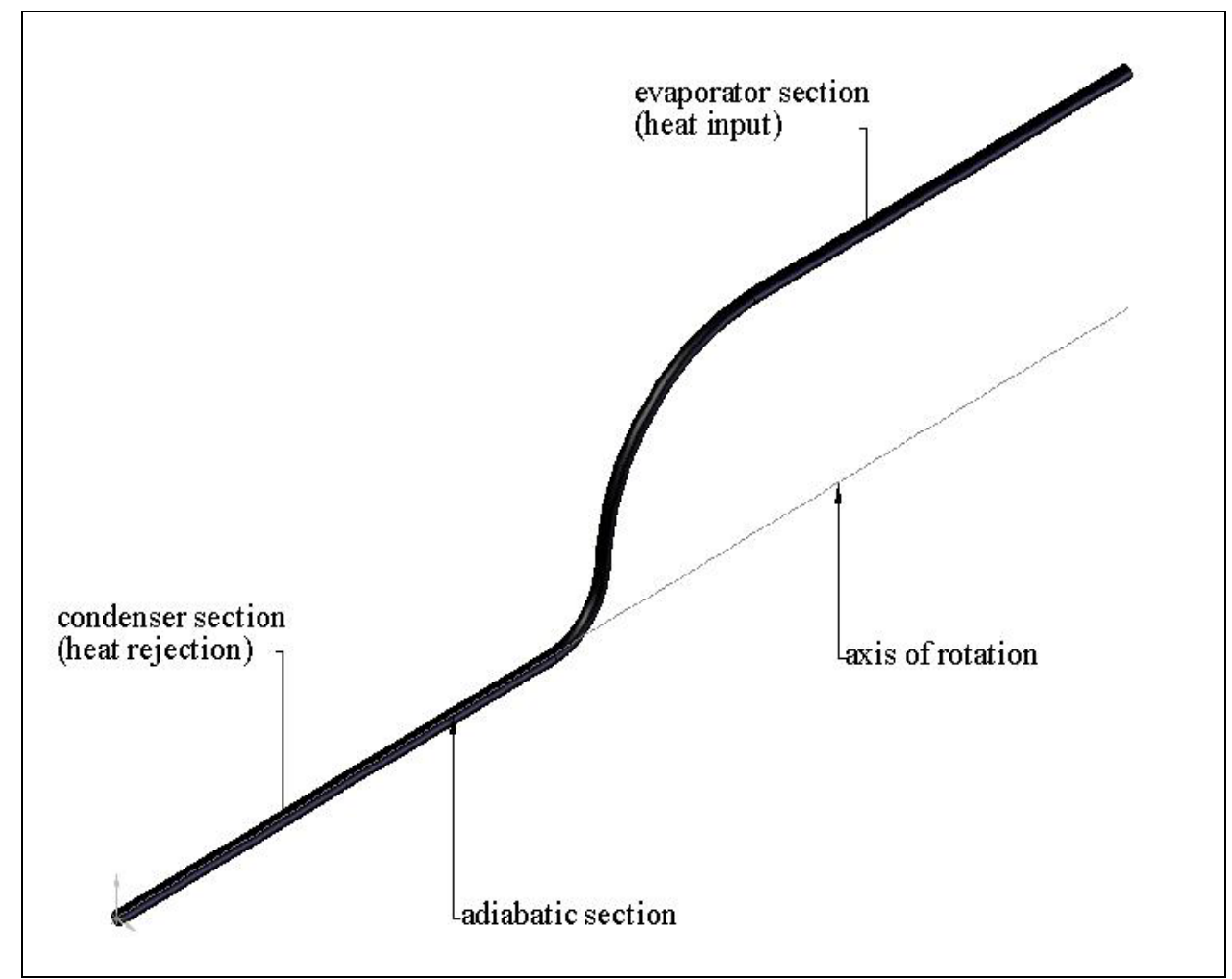

Figure 1.5. The proposed heat pipe geometry.

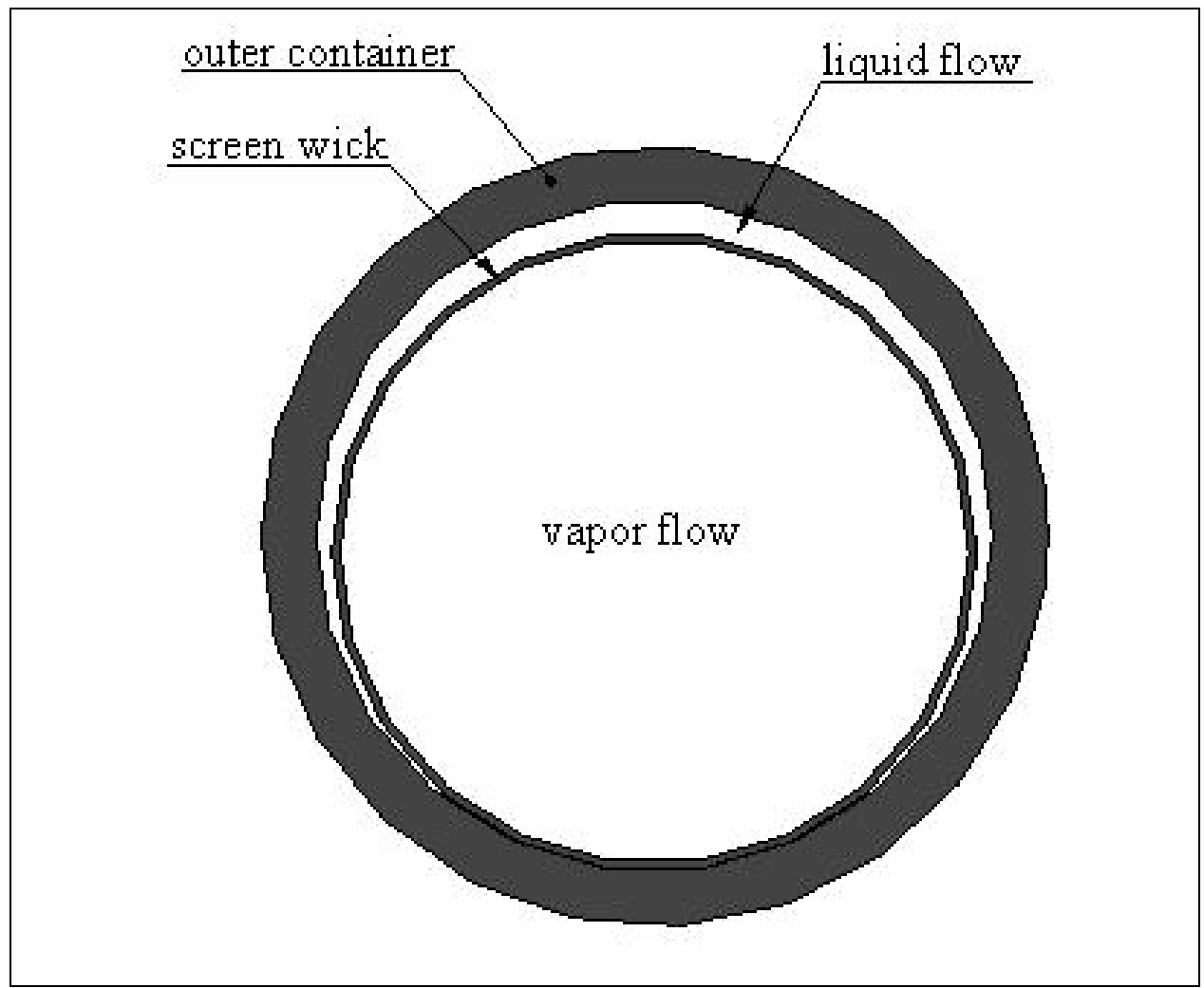

Figure 1.6. Cross-sectional view of the rotating heat pipe. 
allows the heat pipe to be used in stationary operation (with no rotation). The unique geometry of the heat pipe allows the evaporator section to be in contact with heat sources that are located at some distance from the axis of rotation, while the condenser section can be easily coupled to an on-axis heat sink. The proposed heat pipe geometry could find applications in cooling various types of rotating machinery. The most obvious applications are in cooling the windings in the rotor of an electric generator or an electric motor.

Using the proposed heat pipe in applications where off-axis heat sources must be cooled offers many advantages when compared to the use of conventional rotating and revolving heat pipes. For example, in the case of superconducting rotating machines operating at cryogenic temperatures, external heat in-leak, which occurs at the periphery of the machine, is a significant portion of the total heat load that must be removed from the machine to ensure proper operation (Nerowski et al. 2004; Urbahn et al. 2004). While the evaporator section of a revolving heat pipe could easily be placed in contact with the off-axis heated components of a rotating machine, coupling the eccentrically rotating condenser section to a stationary on-axis heat sink can be difficult. The on-axis condenser section of a rotating heat pipe, on the other hand, could more easily be coupled to a heat sink. Coupling the evaporator section of the rotating heat pipe to the off-axis heat sources, however, often requires longer conduction heat transfer paths though solid components of the machine. This conductive cooling can introduce undesirable temperature gradients, as well as raise the operating temperature of the machine's components well above the temperature of the heat sink. From these arguments, the advantages of the heat pipe shown in Fig. 1.5 are realized. 


\section{Literature Review}

A review of articles in the open literature related to the rotating heat pipe proposed in the previous chapter will be presented here. In subsequent chapters, a numerical model of the rotating heat pipe will be developed, and an experimental apparatus for testing a prototype heat pipe will be discussed. To provide a sufficient background for a discussion of the numerical and experimental investigations of the proposed heat pipe, the articles reviewed in this chapter are concerned with modeling of both stationary and rotating heat pipes, experimental studies of rotating and revolving heat pipes, and applications of rotating and revolving heat pipes.

\subsection{Modeling of Stationary Heat Pipes}

Since the invention of the heat pipe in 1963 (Grover et al. 1964), numerous mathematical models of heat pipe operation have been developed. These investigations range in complexity from simple state-to-state thermodynamic analyses (Khalkhali et al. 1999; Richter and Gottschlich 1994; Zuo and Faghri 1997) to transient, multidimensional computational models of the fluid flow and heat transfer in the liquid and vapor phases (Cao and Chang 1997; Cao and Faghri 1991; Cao and Faghri 1993; Tournier and El-Genk 1994; Tournier and El-Genk 1996; Tournier and El-Genk 2003).

Cotter was the first to present a complete analysis of the heat pipe (Cotter 1965). Cotter's analysis focused on a steady state pressure balance in the heat pipe. The pressure drops in the liquid and vapor phases of the working fluid were calculated using one-dimensional, laminar, incompressible flow formulations, and were expressed as a function of the heat transfer rate. The sum of the liquid and vapor phase pressure drops was then set equal to the maximum capillary pressure difference that can be supported by 
the wick. This equality yields the heat transfer rate that produces a capillary limit. The vapor flow analysis by Cotter was restricted to cases of either small or large evaporation and condensation rates (or equivalently heat fluxes) in the evaporator and condenser sections of the heat pipe. A more detailed vapor flow analysis was performed by Busse (Busse 1967), which is valid provided that the vapor flow is steady, laminar, and incompressible. The pressure balance approach for a calculation of the capillary limit, along with empirical and analytical expressions for the entrainment limit (Prenger 1983; Tien and Chung 1979), have been used to develop the steady state heat pipe analysis code HTPIPE (Woloshun et al. 1988). HTPIPE was the first comprehensive heat pipe design and analysis code to be developed. HTPIPE allows for either a calculation of the capillary, entrainment, sonic, and boiling limits, or a prediction of the pressure versus length curves for the liquid and vapor phases. The vapor flow analysis in HTPIPE is an extension of Busse's analysis, allowing for the prediction of turbulent flow in the condenser and adiabatic sections, as well as compressible flow in the adiabatic section.

Although HTPIPE and the associated references used in the code development are valuable tools for the design and analysis of heat pipes operating at steady state, many heat pipe designers have found that more detailed analyses of the fluid flow and heat transfer in the heat pipe are required. Some particular applications requiring more detailed analyses are the prediction of heat pipe performance under the influence of transients (Jang et al. 1991), heat pipes with multiple heat sources and sinks (Faghri et al. 1991a; Faghri et al. 1991b), heat pipes with non-uniform heat distributions and unconventional shapes (Cao and Faghri 1991; Cao and Faghri 1993), and start-up of liquid metal heat pipes from a frozen state (Issacci et al. 1991; Tournier and El-Genk 
1994; Tournier and El-Genk 1996; Tournier and El-Genk 2003). Most of these heat pipe models employ either two or three-dimensional finite-difference or finite-element solutions of the equations governing the fluid flow and heat transfer. One notable contribution to the field of heat pipe modeling is the Heat Pipe Transient Analysis Model (HPTAM), which has become a valuable tool when working with liquid metal heat pipes, where start-up from the frozen state is an important design consideration (Tournier and El-Genk 1994; Tournier and El-Genk 1996; Tournier and El-Genk 2003).

\subsection{Modeling of Rotating and Revolving Heat Pipes}

\subsubsection{Liquid Flow Modeling in Rotating Heat Pipes}

Rotating, wickless heat pipes were first proposed in 1969 by Gray (Gray 1969). Investigations of these wickless heat pipes have found that the liquid flow can be classified into different flow patterns depending on the magnitude of the centrifugal force relative to gravity (Peterson and Wu 1993). For low rotation speeds (where gravity dominates), the liquid will pool at the bottom of the heat pipe, leading to local variations in wall temperature and heat transfer coefficient around the circumference of the condenser and evaporator sections (Krivosheev et al. 1979). As the rotation speed is increased, a stable uniform annulus is formed around the circumference of the heat pipe. Theoretical studies have been used to determine the point where transition from pool flow to annular flow occurs (Baker et al. 2001; Lin and Groll 1996). These studies show that the transition from pool flow to annular flow depends on the relative magnitude of the centrifugal force to gravity (the Froude number) and the percentage of the heat pipe volume filled with liquid. For most heat pipes, annular flow can be maintained when the centrifugal force is twenty times greater than gravity. 
Although some models of the condensation process in heat pipes operating in the pool flow regime have been developed (Khmelev and Shevel 1991), a majority of the predictive tools developed have focused on the heat pipes operating in the annular flow regime. For heat pipes operating in the annular flow regime, accurate predictions of the condensation process in the condenser section are needed. A determination of the liquid film thickness is needed because the thermal resistance in the heat pipe will increase as thicker liquid films are developed in the condenser section. Additionally, the condensation process can present a heat transfer limitation. As discussed in the previous section, a component of the centrifugal force is typically used to drive the liquid out of the condenser. If this driving force is insufficient to drive as much mass out of the condenser as is condensing at the liquid-vapor interface, the rotating heat pipe can fail due to a condensation limit (Faghri 1995).

Daniels and Al-Jumaily applied a Nusselt-type analysis to the rotating liquid film in the condenser section of a rotating, tapered heat pipe (Daniels and Al-Jumaily 1975). The analysis assumed steady, laminar film flow with a linear temperature variation over the thin liquid film. The interfacial shear stress and mass flux were considered in the analysis, however, the axial pressure gradient in the vapor phase was ignored. The Nusselt condensation analysis uses scaling arguments to reduce the momentum equations to a form that can be solved easily. Acceleration in the liquid flow direction is ignored, while both convection and diffusion are ignored in the cross-stream direction. Using their analysis, Daniels and Al-Jumaily developed expressions for the liquid layer thickness and the heat transfer coefficient in the condenser section. The condensation analysis was extended by Marto to include the effect of pressure drop in the vapor phase 
(Marto 1976). This thin-film analysis has been used to study internally finned rotating heat pipes (Lin and Faghri 1999; Salinas and Marto 1991) and radially rotating heat pipes (Cao and Chang 1997).

The film condensation analysis has also been used to calculate the film thickness in the adiabatic and evaporator sections of axially rotating heat pipes (Li et al. 1993; Song et al. 2003). These studies found that accurate predictions of the overall thermal resistance could only be made if the model determined how the liquid charge, under the influence of the centrifugal forces, is redistributed in the heat pipe. In addition, the model must consider how the heat transfer is affected by over-filling the heat pipe with liquid. Finally, Song et al. found that heat transfer augmentation by natural convection in the liquid film of the evaporator section (a liquid film heated from 'below') influences the overall thermal resistance and must be included in the model (Song et al. 2003).

\subsubsection{Complete Models of Rotating Heat Pipes}

In the analyses of the liquid film, the shear stress at the liquid-vapor interface was estimated using the friction factor for fully-developed vapor flow in a smooth tube. A two-dimensional finite-difference model of the vapor flow by Faghri et al., however, shows that the vapor flow is significantly affected by rotation of the heat pipe (Faghri et al. 1993). At high rotation rates, Faghri et al. showed that flow reversal near the centerline of the heat pipe was possible, and that the friction factor for the vapor flow in the rotating heat pipe was higher than the friction factor for flows in non-rotating tubes.

Complete two-dimensional analyses of the liquid and vapor flows were performed by Harley and Faghri for a wickless rotating heat pipe with an ideal fluid loading (Harley and Faghri 1995), and by Ismail and Miranda (Ismail and Miranda 1997) and Machado 
and Miranda (Machado and Miranda 2003) for a rotating heat pipe with a homogenous wick. The analysis by Harley and Faghri coupled the two-dimensional finite-difference vapor flow model to the Nusselt-type analysis for the liquid layer. The liquid layer analysis was restricted to an ideal fluid loading (a fluid charge that gives zero film thickness at the evaporator and condenser end-caps). Ismail and Miranda and Machado and Miranda used a two-dimensional finite-difference model to analyze both the vapor flow and the liquid flowing in the wick. These analyses show that in a heat pipe with a homogeneous wick the liquid flow is not influenced by axial rotation.

A complete computational model of a rotating heat pipe, based on the full NavierStokes and energy equations applied to the liquid and vapor phases, was formulated by Baker et al. (Baker et al. 1999). The proposed model treated the liquid and vapor in the rotating heat pipe as a single-domain problem, and the Volume of Fluid method was employed to determine the location of the liquid-vapor interface. Although the governing equations and boundary conditions for an axially rotating wickless heat pipe were formulated and the model was described in detail, results of the model simulations were not given.

\subsubsection{Revolving and Radially Rotating Heat Pipes}

With the exception of the works by Curtila and Chataing (Curtila and Chataing 1984) and Castle et al. (Castle et al. 2000; Castle et al. 2001), attempts at characterizing the performance of revolving heat pipes have been restricted to experimental studies. Curtila and Chataing analyzed a revolving smooth-walled heat pipe with a thermal resistance network, however, the results of the model were not compared to their experimental data (Curtila and Chataing 1984). Castle et al. determined the capillary limit in a revolving 
heat pipe with helical grooves on the inner wall of the pipe by calculating the pressure drop in the liquid flowing in each groove (Castle et al. 2000; Castle et al. 2001). Heat transfer in the liquid, however, was not modeled for the helically grooved heat pipe.

Radially rotating heat pipes have been modeled mainly through an investigation of the pressure and temperature variations in the vapor phase. Ling and Cao (Ling and Cao 2000) and Ling et al. (Ling et al. 2001) have used one-dimensional models to estimate the variation in saturation temperature with length in a radially rotating heat pipe.

\subsection{Experiments With Rotating and Revolving Heat Pipes}

\subsubsection{Liquid Flow Regimes in Rotating Heat Pipes}

The flow regimes in low-speed rotating heat pipes have been extensively characterized in experimental studies. A number of clear heat pipes have been built and tested, allowing for visual observation of the transition from pool flow (or stratified flow) to annular flow (or rimming) (Baker et al. 2001; Katsuta et al. 1984; Lin and Groll 1996; Nakayama et al. 1984; Takahashi et al. 1995a). The heat transfer coefficients in the evaporator and condenser sections during stratified flow and in annular flow have also been measured (Jian et al. 1987; Jian et al. 1990; Krivosheev et al. 1979; Reddy et al. 1987; Reddy et al. 1990). The visualization and heat transfer studies conclude that the liquid flow and corresponding heat transfer can be categorized into four distinct regimes (Peterson and Wu 1993):

1. Pool flow: At low rotation speed ( $<10 \mathrm{~g}$ centrifugal acceleration) gravity dominates and a liquid pool forms in the bottom of the heat pipe. A small portion of liquid is dragged out of the pool by the moving wall and forms a thin film on the upper portion of the pipe. Heat transfer coefficients in the 
evaporator and condenser sections are at their highest level in the pool flow regime.

2. Partial annular flow: As the rotational speed is increased to moderate speeds (10 g - $20 \mathrm{~g}$ ) the liquid film on the upper portion of the pipe becomes thicker and the size of the liquid pool decreases. Heat transfer coefficients in the partial annular flow regime are comparable in size to the pool flow regime.

3. Annular flow: As the rotational speed is increased, a critical rotation speed is reached (typically $\sim 20 \mathrm{~g}$ ) where the liquid pool disappears and the liquid forms a stable annulus with uniform thickness around the circumference of the heat pipe. When the critical rotation speed is reached, the heat transfer coefficients in the condenser and evaporator sections decrease to a low level (typically an order of magnitude lower than the heat transfer coefficients in pool flow).

4. Hysteresis: Once stable annular flow has been established, the rotation speed can be reduced to a level well below the critical rotation speed while still maintaining annular flow. As the rotation speed is decreased below the critical speed, a destruction speed is eventually reached (typically $\sim 10 \mathrm{~g}$ ) where the structure reverts back to pool flow.

The heat transfer studies show that heat transfer coefficients are highest when a large liquid pool is formed at the bottom of the heat pipe. Most investigators indicate that in the pool flow regime, a majority of the heat transfer through the liquid takes place in the thin liquid layer lining the upper portion of the pipe and that the pool contributes little to the overall thermal resistance (Reddy et al. 1987). Once annular flow is established, the 
thicker liquid layer on the upper portion of the pipe reduces the evaporator and condenser heat transfer coefficients.

\subsubsection{High Speed Rotating Heat Pipes}

For higher rotation speeds, with the heat pipe operating in the annular flow regime, Daniels and Al-Jumaily investigated a $325 \mathrm{~mm}$ long, $30 \mathrm{~mm}$ diameter rotating heat pipe with a 2 degree internal taper from the condenser end to the evaporator end (Daniels and Al-Jumaily 1975). The heat pipe was rotated up to $1200 \mathrm{rpm}$ and was tested with heat loads up to $2000 \mathrm{~W}$. The heat pipe was tested with Arcton 113, Arcton 21, and water working fluids. The agreement between experimental results and the Nusselt analysis of the condensation process was achieved when an optimal fluid charge was used. The experimental investigation showed that the results were sensitive to the fluid charge. With an insufficient amount of fluid in the heat pipe, the working fluid would not circulate, and the heat pipe required a large temperature difference between the condenser and evaporator sections to transfer heat. With an overfilled heat pipe, the liquid layer thickness in the evaporator and condenser sections was thicker than the optimal, increasing the thermal resistance across the liquid layer.

A test rig and heat pipe for use while rotating at speeds up to 40,000 rpm are described in the papers by Streby et al. (Streby et al. 1996) and Ponnappan and He (Ponnappan and He 1998). Heat pipes with a $2.54 \mathrm{~cm}$ internal diameter, a 1 degree taper in the condenser section, and a total length of $25 \mathrm{~cm}$ were tested. Water and methanol working fluids were used in the heat pipes. Tests were performed with total power inputs from 250 to $1300 \mathrm{~W}$, at rotational speeds ranging from 5000 to 30,000 rpm. Although the heat pipes operated successfully and met all of the performance requirements of the 
investigators, the agreement between the test results and the Nusselt-type condensation analysis was poor. The conclusion was made that the scaling arguments used in the Nusselt analysis are not applicable under the influence of the centrifugal acceleration levels observed in the tests (reported acceleration levels were from 1000 to $9000 \mathrm{~g}$ ).

In experiments by Song et al., water filled heat pipes with $2.54 \mathrm{~cm}$ outer diameters and $40 \mathrm{~cm}$ total lengths, both with and without an internal taper on the condenser section, were rotated up to $4000 \mathrm{rpm}$ (Song et al. 2004). The data from the experiments of Ponnappan and He and Song et al. were analyzed with a modified film condensation analysis (Song et al. 2003; Song et al. 2004). Modifications made to the condensation analysis were (1) the accounting for excess fluid loading (above the optimal) in the heat pipe and (2) the inclusion of natural convection in the liquid film in the evaporator section. The ability of the model to predict the performance of the rotating heat pipe was assessed by comparing how well the model predicted the average temperature difference between the evaporator and condenser section with changes in heat load, rotation speed, and fluid loading. Reasonable agreement between test data and model predictions was achieved for the high speed axially rotating heat pipes, with the model under predicting the temperature difference (by as much as $15 \%$ ) for the tapered heat pipes and over predicting the temperature difference (by as much as $40 \%$ ) for the cylindrical heat pipes. More importantly, by accounting for excess fluid loading and natural convection in the evaporator film, the modified analysis was able capture the general trends of decreasing thermal resistance with increases in rotation speed and decreases in fluid loading. These were trends that could not be explained using models that did not include these modifications (Li et al. 1993). 
The use of an internal taper in a rotating or revolving heat pipe can increase fabrication costs, and in the case of very long heat pipes, the machining operation to form the tapered wall can be prohibitively expensive. For these reasons, alternate methods of increasing the flow rate of liquid in the rotating heat pipe have been investigated. Successful methods of augmenting the heat transfer performance of rotating and revolving heat pipes include inserting a long-pitch spring into the heat pipe container (Lee and Kim 2001; Shimizu and Yamazaki 1987) and the use of tubes with helical grooves on the inner wall (Castle et al. 2001). These studies showed, both through modeling and experimental studies, that the helically-grooved wick geometry and the coil-inserted wick structure produce a pumping effect on the liquid flow that increases with rotation speed.

\subsubsection{Radially Rotating Heat Pipes}

Ling et al. tested sodium filled miniature heat pipes in a radially rotating position. The heat pipes tested were $85 \mathrm{~mm}$ long with diameters of 1.5 and $2 \mathrm{~mm}$. The rotation rate was varied from 1800 to $3600 \mathrm{rpm}$. The experimental investigation confirmed the results obtained from their modeling study. Both the model and experiments suggested that the temperature variations along the length of the heat pipe were affected by the centrifugal force (or the rotation rate) and the friction force on the vapor (or the heat pipe

diameter). A larger temperature difference between the evaporator and condenser sections was predicted and observed for heat pipes with a smaller diameter and for heat pipes rotating at higher speeds.

Waowaew et al. also tested radially rotating heat pipes and developed a correlation to predict the performance (Waowaew et al. 2003). The heat pipes tested had inner 
diameters of 11, 26, and $50.4 \mathrm{~mm}$ and were filled with water, ethanol, and R123. The length of the heat pipes varied from 5 to 40 times the diameter. A correlation was developed relating the axial heat flux to the aspect ratio, rotational acceleration and working fluid properties. Temperature variations along the length of the heat pipes, however, were not reported.

\subsubsection{Revolving Heat Pipes}

Flow visualization and heat transfer studies have also been performed on revolving heat pipes. Gi and Maezawa (Gi and Maezawa 1990) conducted visualization studies with a $37 \mathrm{~mm}$ diameter, $500 \mathrm{~mm}$ long glass tube mounted parallel to the axis of rotation with a distance of $200 \mathrm{~mm}$ from the axis of rotation to the centerline of the tube. The flow pattern in the tube was observed for rotation speeds from 0 to $350 \mathrm{rpm}$ and further characterized in terms of a dimensionless acceleration (Froude number, $F r=\omega^{2} d / g$ ). The visual studies show that when the centrifugal acceleration is less than gravity ( $F r<1$ ), the liquid forms a pool on the lower portion of the revolving tube. For $F r \sim 1$, the liquid experiences a zero g condition at the top of the revolution. The visualization studies show that when the centrifugal acceleration is comparable in size to gravity, the liquid splashes in the tube. As the rotation speed is increased beyond $F r=1$, the liquid forms a pool against the wall farthest from the axis of rotation.

Heat transfer studies with revolving heat pipes indicate that the heat transfer coefficients in the evaporator and condenser reach a maximum near $F r=1$ (due to the splashing of the liquid), then fall to a nearly constant level at higher rotation speeds (Curtila and Chataing 1984; Gi and Maezawa 1990; Niekawa et al. 1981; Pokorny et al. 1984). Furthermore, studies have shown that using multiple grooves on the inner surface 
of the revolving heat pipe significantly increases the heat transfer coefficients compared to smooth-walled revolving heat pipes (Castle et al. 2000; Castle et al. 2001; Klasing et al. 1999; Niekawa et al. 1981).

\subsection{Applications of Rotating and Revolving Heat Pipes}

Rotating and revolving heat pipes have been used as part of the thermal control system in a wide variety of rotating equipment. The feasibility of using rotating, radially rotating, and revolving heat pipes in cooling various components of electrical machines such as electric motors and generators has been studied (Bailey and Thronton 1978; Giessler et al. 1987; Oslejsek and Polasek 1976). Ponnappan and Leland retrofit the shaft of a high speed permanent magnet alternator with a hollow shaft which operated as a rotating heat pipe (Ponnappan and Leland 1998). For identical operating conditions, the alternator equipped with the rotating heat pipe operated at significantly lower temperatures than the conventional alternator. Thoren proposed using stationary and rotating, revolving, and radially rotating heat pipes to cool various components in electric motors (Thoren 1984). The study showed that heat pipes could be effective in both opentype and totally enclosed induction motors.

Niekawa et al. tested a revolving heat pipe for use in a rotary heat pipe heat exchanger (Niekawa et al. 1981). The heat exchanger was being developed to improve the efficiency of waste heat recovery from exhaust gases in industrial applications. In this application rotating heat pipe heat exchangers were shown to offer the advantages of variable capacity (by varying the rotation speed of the revolving heat pipes) and easy access for cleaning and de-fouling the heat transfer surfaces. 
Rotating heat pipes have been used in the horizontal and vertical position to cool the bearings and seals of a fan in a lift bell furnace (Pokorny et al. 1984). Rotating heat pipes have also been proposed to remove the heat produced in high speed cutting operations. Judd et al. used a rotating heat pipe in cooling the spindle bearings in a milling machine (Judd et al. 1994), while Jen et al. investigated the possibility of using a heat pipe to cool the tip of a drill bit (Jen et al. 2002).

Radially rotating heat pipes have been used successfully in turbine blade cooling applications (Langston and Faghri 1995; Ling and Cao 2000; Ling et al. 2001) and to cool disk brakes in automotive applications (Maezawa et al. 1984).

As mentioned in the introduction, the rotating heat pipe that incorporates the Sshaped curve has many beneficial features when compared to the straight heat pipes studied in the past. In particular, the proposed heat pipe would be an attractive option in applications of rotating machinery where heat sources are located at some distance from the axis of rotation and coupling the revolving condenser section to the heat sink is difficult. 


\section{Rotating Heat Pipe Model}

A two-dimensional finite-difference model of the liquid flow in the rotating heat pipe is outlined in this section. For the S-shaped heat pipe, a simple model of the liquid flow (Jankowski et al. 2004), based on the boundary layer equations applied to the liquid layer, shows that at low rotation rates $(<100 \mathrm{rpm})$ the liquid fills the annulus and the wick is used to pump liquid from the condenser toward the evaporator. At higher rotation rates, however, the simplified model shows that the liquid recedes from the wick and forms a variable thickness film in contact with the inner wall of the heat pipe. As suggested by the simplified boundary layer analysis, the finite-difference model of the liquid flow must have the capability to predict the location of multiple wet-points and to determine the transition from the wicked to non-wicked operating regimes.

The goal here is to develop, verify, and validate a design code that can be used to investigate the factors affecting the performance of the rotating heat pipe. The liquid flow model developed here is based closely on the work of Song et al. (Song et al. 2003), however, whereas the convective terms and the Coriolis forces are ignored in previous investigations of the liquid flow in axially rotating heat pipes, these terms are retained throughout the formulation here. Furthermore, while previous models of rotating heat pipes (Daniels and Al-Jumaily 1975; Li et al. 1993; Song et al. 2003) have ignored temperature and pressure variations in the vapor phase, the present investigation couples the liquid flow to a one-dimensional vapor flow model. The inclusion of these terms represents an extension of the previous models. These extensions allow the model to be used for heat pipes with more complex shapes and in situations where these forces affect the overall thermal resistance of the heat pipe. 


\subsection{GEOMETRY}

The geometry is represented in the mathematical model using the notation shown in Fig. 3.1. The liquid is viewed as a thin film of thickness $\delta$ in contact with the inner wall of the heat pipe. The coordinate axes are located at the end of the condenser section, with $r$ directed radially outward, the circumferential direction is labeled $\theta$, and with the axial coordinate $x$ directed toward the adiabatic and evaporator sections. With the inner radius of the heat pipe located at $r=R$, the liquid-vapor interface is positioned at $r=R-\delta$. At a given axial location, the angle between the axis of rotation and the $r$ axis is labeled $\alpha$, and the distance from the axis of rotation to the centerline of the heat pipe is defined by $d$. At $x=0$ the inner radius of the heat pipe is defined as $R_{0}$, and the liquid layer thickness is $\delta_{0}$. In general, the inner radius of the heat pipe, the liquid layer thickness, the angle with the axis of rotation, and the distance from the axis of rotation

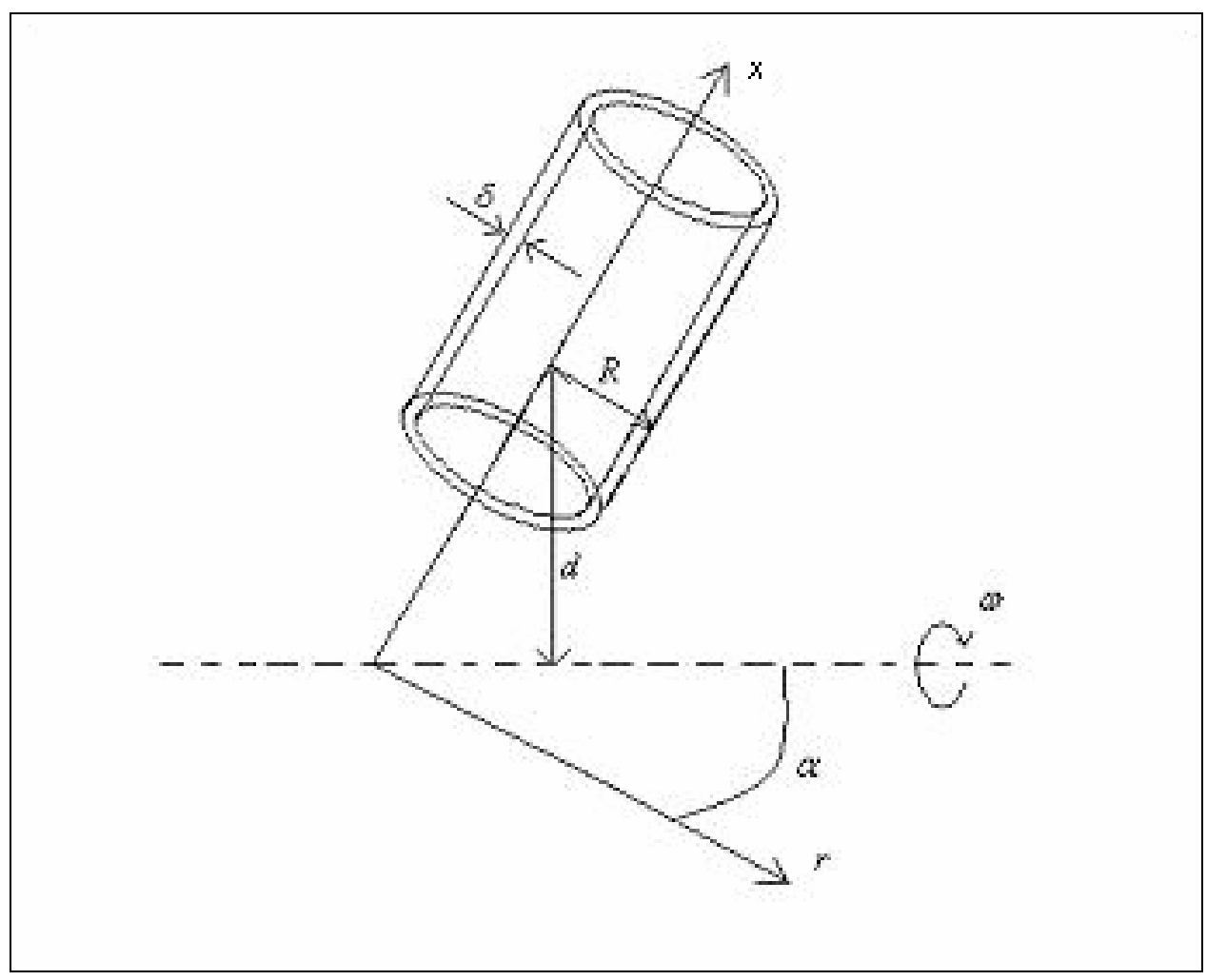

Figure 3.1. An illustration of the liquid layer and parameters used in the model. 
are functions of the axial coordinate, or $R=R(x), \delta=\delta(x), \alpha=\alpha(x)$, and $d=d(x)$. The length of the condenser, adiabatic, and evaporator sections are defined as $L_{c}, L_{a}$, and $L_{e}$, respectively, and the total length of the heat pipe is $L_{\text {tot }}=L_{c}+L_{a}+L_{e}$.

\subsection{Assumptions}

The liquid flow is treated as steady, laminar, and two-dimensional. Throughout the modeling, symmetry about the longitudinal axis is assumed $(\partial / \partial \theta=0)$. Furthermore, the liquid is modeled as Newtonian, with constant viscosity, thermal conductivity, and specific heat. Uniform heat addition per unit length into the evaporator section and uniform heat rejection per unit length from the condenser section are assumed. To develop a fast and economical rotating heat pipe design code, the diffusion terms in the stream-wise direction (the $x$-direction) are ignored. This assumption allows for a spacemarching procedure to be used, and is valid in the absence of reverse flow in the liquid (Patankar and Spalding 1972). This procedure does not, however, allow for the natural convection in the evaporator to be modeled explicitly. For this reason, the diffusion term in the energy equation is multiplied by a Nusselt number to account for heat transfer augmentation due to the presence of natural convection.

\subsection{Mathematical Model and Numerical Analysis}

\subsubsection{Governing Equations ANd Boundary Conditions}

For the problem depicted in Fig. 3.1, with the assumptions outlined above, the governing equations are (Bird et al. 2002)

$$
\begin{aligned}
& \text { continuity: } \frac{1}{r} \frac{\partial\left(r \mathrm{v}_{r}\right)}{\partial r}+\frac{\partial \mathrm{v}_{x}}{\partial x}=0 \\
& \text { momentum and energy: } \frac{1}{r} \frac{\partial\left(r \mathrm{v}_{r} \phi\right)}{\partial r}+\frac{\partial\left(\mathrm{v}_{x} \phi\right)}{\partial x}=\frac{\Gamma}{r} \frac{\partial}{\partial r}\left(r \frac{\partial \phi}{\partial r}\right)+S^{\phi}+\nabla p^{\phi},
\end{aligned}
$$


where for

$$
\begin{array}{r}
\text { r-momentum: } \phi=\mathrm{v}_{r}, S^{\phi}=\frac{\mathrm{v}_{\theta}^{2}}{r}-\frac{\mu}{\rho} \frac{\mathrm{v}_{r}}{r^{2}}+2 \omega \mathrm{v}_{\theta} \sin \alpha+\omega^{2}(d+r) \sin \alpha \\
\nabla p^{\phi}=-\frac{1}{\rho} \frac{\partial p}{\partial r}, \text { and } \Gamma=\frac{\mu}{\rho}
\end{array}
$$

$\theta$-momentum: $\phi=\mathrm{v}_{\theta}, S^{\phi}=-\frac{\mathrm{v}_{r} \mathrm{v}_{\theta}}{r}-\frac{\mu}{\rho} \frac{\mathrm{v}_{\theta}}{r^{2}}-2 \omega\left(\mathrm{v}_{r} \sin \alpha+\mathrm{v}_{x} \cos \alpha\right)$,

$$
\begin{aligned}
& \qquad p^{\phi}=0 \text {, and } \Gamma=\frac{\mu}{\rho} \\
& \text { x-momentum: } \phi=\mathrm{v}_{x}, S^{\phi}=\omega^{2} d \cos \alpha+2 \omega \mathrm{v}_{\theta} \cos \alpha, \nabla p^{\phi}=-\frac{1}{\rho} \frac{\partial p}{\partial x} \text {, and } \Gamma=\frac{\mu}{\rho} \\
& \text { energy: } \quad \phi=T, S^{\phi}=0, \nabla p^{\phi}=0 \text {, and } \Gamma=\frac{k N u}{\rho C_{p}}
\end{aligned}
$$

The boundary conditions include the no-slip condition, where the velocities $\left(\mathrm{v}_{r}, \mathrm{v}_{\theta}\right.$, and $\left.\mathrm{v}_{x}\right)$ are set to zero at the solid surfaces $\left(r=R, x=0\right.$, and $\left.x=L_{\text {tot }}\right)$. The heat fluxes to the wall of the evaporator and condenser sections

$$
q^{\prime \prime}=-k N u\left(\nabla T \cdot \mathbf{n}_{\mathbf{w}}\right)_{r=R},
$$

are assumed constant, where $N u$ is the Nusselt number in the liquid, and $\mathbf{n}_{\mathbf{w}}=\left(\mathbf{e}_{r}-R^{\prime} \mathbf{e}_{x}\right) / \sqrt{1+R^{\prime 2}}$ is a unit vector normal to the heat pipe wall with primes representing differentiation with respect to $x$. In each section of the heat pipe, the heat fluxes can be related to the power throughput as

$$
q^{\prime \prime}= \begin{cases}Q / A_{c} & \text { condenser section } \\ 0 & \text { adiabatic section } \\ -Q / A_{e} & \text { evaporator section }\end{cases}
$$


where $A_{c}$ and $A_{e}$ are the surface areas of the condenser and evaporator sections, respectively. Additionally, the end-caps are modeled as insulated,

$$
\partial T /\left.\partial x\right|_{x=0}=\partial T /\left.\partial x\right|_{x=L_{\text {tot }}}=0
$$

The boundary conditions at the liquid-vapor interface $(r=R-\delta)$ include a temperature specification, an energy balance, and a normal stress jump condition written as (Slattery 1999)

$$
\begin{gathered}
T=T_{s} \\
\rho h_{f g}(\mathbf{v} \cdot \mathbf{n})=-k N u(\nabla T \cdot \mathbf{n}) \\
-2 H \sigma\left(n_{r}^{2}+n_{x}^{2}\right)=\rho\left(1-\rho / \rho_{v}\right)(\mathbf{v} \cdot \mathbf{n})^{2}\left(n_{r}^{2}+n_{x}^{2}\right)+\tau_{r r} n_{r}^{2} \\
+2\left(\tau_{r x}-\tau_{r x, v}\right) n_{r} n_{x}+\tau_{x x} n_{x}^{2}+\left(p-p_{v}\right)
\end{gathered}
$$

When the liquid fills the annular space and the wick is full (referred to as wicked operation), the no-slip conditions at the wick $(r=R-\delta)$ are also imposed: $\mathrm{v}_{\theta}=\mathrm{v}_{x}=0$. However, when the wick is empty and the liquid has a free-surface at $r=R-\delta$ (referred to as non-wicked operation), the tangential stress balances,

$$
\begin{gathered}
\tau_{r \theta} n_{r}+\tau_{x \theta} n_{x}=0, \\
\tau_{r r} n_{r} n_{x}+\left(\tau_{r x}-\tau_{r x, v}\right)\left(n_{z}^{2}-n_{r}^{2}\right)-\tau_{x x} n_{r} n_{x}=0,
\end{gathered}
$$

are imposed at the free-surface along with Eqs. (3.10), (3.11), and (3.12), where the unit normal vector at the liquid-vapor interface is given by

$$
\mathbf{n}=n_{r} \mathbf{e}_{r}+n_{x} \mathbf{e}_{x}=\left[\mathbf{e}_{r}+\left(\delta^{\prime}-R^{\prime}\right) \mathbf{e}_{x}\right] / \sqrt{1+\left(\delta^{\prime}-R^{\prime}\right)^{2}}
$$

and the mean curvature of the interface is 


$$
H=\frac{1}{2(\overline{\bar{R}} \bar{\delta})}\left[(\bar{R}-\bar{\delta})\left(\bar{R}^{\prime \prime}-\bar{\delta}^{\prime \prime}\right)-\left(\bar{R}^{\prime}-\bar{\delta}^{\prime}\right)^{2}-1\right]\left[1+\left(\bar{R}^{\prime}-\bar{\delta}^{\prime}\right)^{2}\right]^{-3 / 2}
$$

In these free-surface conditions, the stress components in the liquid are expressed as $\tau_{x x}=-2 \mu\left(\partial \mathrm{v}_{x} / \partial x\right), \tau_{r r}=-2 \mu\left(\partial \mathrm{v}_{r} / \partial r\right), \tau_{r \theta}=-\mu\left(\partial \mathrm{v}_{\theta} / \partial r-\mathrm{v}_{\theta} / r\right), \tau_{\theta x}=-\mu\left(\partial \mathrm{v}_{\theta} / \partial x\right)$ and $\tau_{r x}=-\mu\left(\partial \mathrm{v}_{r} / \partial x+\partial \mathrm{v}_{x} / \partial r\right)$. The shear stress in the vapor is written as $\tau_{r x, v}=f \rho_{v}\left\langle\mathrm{v}_{v}\right\rangle^{2} / 8$, where $\left\langle\mathrm{v}_{v}\right\rangle$ is the average vapor velocity in the axial direction, and $f$ is the Darcy friction factor (Fox and McDonald 1992).

\subsubsection{Coordinate Transformations and Dimensionless Parameters}

The coordinates are transformed by defining the independent variables as

$$
\xi=\left(r / R_{0}-\bar{R}+\bar{\delta}\right) / \bar{\delta} \text { and } \chi=x / R_{0}
$$

where $\bar{\delta}=\bar{\delta}(\chi)=\delta / R_{0}$ and $\bar{R}=\bar{R}(\chi)=R / R_{0}$

With this transformation, the liquid-vapor interface is located at $\xi=0$ and the wall of the heat pipe corresponds to $\xi=1$, regardless of the liquid layer thickness $\bar{\delta}(\chi)$ or the shape of the heat pipe wall $\bar{R}(\chi)$. The transformation does, however, introduce $\bar{\delta}, \bar{R}$, and their derivatives with respect to $\chi$ into the governing equations and boundary conditions.

Velocity, temperature, and rotation speed are made dimensionless by

$$
\mathbf{u}=\mathbf{v} / \mathrm{v}_{c h}, \Lambda=T / T_{c h} \text {, and } \Omega=\omega R_{0} / \mathrm{v}_{c h} \text {, }
$$

where the characteristic velocity and temperature are $\mathrm{v}_{c h}=Q /\left(\rho h_{f g} R_{0}^{2}\right)$ and $T_{c h}=Q /\left(k R_{0}\right)$. Using these expressions, the governing equations become 
continuity: $\quad \frac{1}{\bar{\delta}(\bar{R}+\xi \bar{\delta}-\bar{\delta})} \frac{\partial}{\partial \xi}\left[(\bar{R}+\xi \bar{\delta}-\bar{\delta}) u_{r}\right]+\frac{\partial u_{x}}{\partial \chi}$

$$
-\frac{\left[\bar{\delta}^{\prime}(1-\xi)-\bar{R}^{\prime}\right]}{\bar{\delta}} \frac{\partial u_{x}}{\partial \xi}=0
$$

momentum and energy: $\frac{1}{\bar{\delta}(\bar{R}+\xi \bar{\delta}-\bar{\delta})} \frac{\partial}{\partial \xi}\left[(\bar{R}+\xi \bar{\delta}-\bar{\delta}) u_{r} \phi-\bar{\Gamma} \frac{(\bar{R}+\xi \bar{\delta}-\bar{\delta})}{\bar{\delta}} \frac{\partial \phi}{\partial \xi}\right]$

$$
+\frac{\partial}{\partial \chi}\left(u_{x} \phi\right)+\frac{\left[\bar{\delta}^{\prime}(1-\xi)-\bar{R}^{\prime}\right]}{\bar{\delta}} \frac{\partial}{\partial \xi}\left(u_{x} \phi\right)=\bar{S}^{\bar{\phi}}+\nabla \Pi^{\bar{\phi}}
$$

where for

r-momentum: $\bar{\phi}=u_{r}, \bar{S}^{\bar{\phi}}=\frac{u_{\theta}^{2}}{(\bar{R}+\xi \bar{\delta}-\bar{\delta})}-\frac{1}{\operatorname{Re}} \frac{u_{r}}{(\bar{R}+\xi \bar{\delta}-\bar{\delta})}+2 \Omega u_{\theta} \sin \alpha$

$$
+\Omega^{2}(\bar{d}+\bar{R}+\xi \bar{\delta}-\bar{\delta}) \sin \alpha, \nabla \Pi^{\bar{\phi}}=-\frac{1}{\bar{\delta}} \frac{\partial \Pi}{\partial \xi} \text {, and } \bar{\Gamma}=\frac{1}{\operatorname{Re}},
$$

$\theta$-momentum: $\bar{\phi}=u_{\theta}, \bar{S}^{\bar{\phi}}=-\frac{u_{r} u_{\theta}}{(\bar{R}+\xi \bar{\delta}-\bar{\delta})}-\frac{1}{\operatorname{Re}} \frac{u_{r}}{(\bar{R}+\xi \bar{\delta}-\bar{\delta})^{2}}$

$$
-2 \Omega\left(u_{r} \sin \alpha+u_{x} \cos \alpha\right), \nabla \Pi^{\bar{\phi}}=0 \text {, and } \bar{\Gamma}=\frac{1}{\mathrm{Re}},(3.2
$$

x-momentum: $\quad \bar{\phi}=u_{x}, \bar{S}^{\bar{\phi}}=\Omega^{2} \bar{d} \cos \alpha+2 \Omega u_{\theta} \cos \alpha$,

$$
\nabla \Pi^{\bar{\phi}}=-\frac{\partial \Pi}{\partial \chi}-\frac{\left[\bar{\delta}^{\prime}(1-\xi)-\bar{R}^{\prime}\right]}{\bar{\delta}} \frac{\partial \Pi}{\partial \xi} \text {, and } \bar{\Gamma}=\frac{1}{\mathrm{Re}},
$$

energy: $\quad \bar{\phi}=\Lambda, \bar{S}^{\bar{\phi}}=0, \nabla \Pi^{\bar{\phi}}=0$, and $\bar{\Gamma}=\frac{N u}{P e}$.

The following dimensionless parameters appear in Eqs. (3.19)-(3.24): the dimensionless pressure $\Pi=p /\left(\rho \mathrm{v}_{c h}^{2}\right)=p / p_{c h}$, the Reynolds number $\operatorname{Re}=\rho \mathrm{v}_{c h} R_{0} / \mu$, and the Péclet number $P e=\rho C_{p} \mathrm{v}_{c h} R_{0} / k$. 
To simplify integration of the governing equations, $x$ and $\xi$-direction velocities ( $u_{x}$ and $u_{\xi}$, respectively) are retained, and the momentum equations are expressed in the $x$ and $\xi$-directions (Faghri et al. 1984). The coordinate transformation in Eq. (3.17) shows that

$$
u_{r}=u_{\xi}-\left[\overline{\delta^{\prime}}(1-\xi)-\bar{R}^{\prime}\right] u_{x}
$$

and $\quad[\xi-$ momentum $]=[r-$ momentum $]+\left[\bar{\delta}^{\prime}(1-\xi)-\bar{R}^{\prime}\right][x-$ momentum $]$.

Finally, with Eqs. (3.25) and (3.26) the governing equations become

continuity:

$$
\begin{aligned}
\frac{1}{\bar{\delta}(\bar{R}+\xi \bar{\delta}-\bar{\delta})} \frac{\partial}{\partial \xi}\left[(\bar{R}+\xi \bar{\delta}-\bar{\delta}) u_{\xi}\right] \\
+\frac{1}{\bar{\delta}(\bar{R}+\xi \bar{\delta}-\bar{\delta})} \frac{\partial}{\partial \chi}\left[\bar{\delta}(\bar{R}+\xi \bar{\delta}-\bar{\delta}) u_{x}\right]=0,
\end{aligned}
$$

momentum and energy: $\frac{1}{\bar{\delta}(\bar{R}+\xi \bar{\delta}-\bar{\delta})} \frac{\partial}{\partial \xi}\left[(\bar{R}+\xi \bar{\delta}-\bar{\delta}) u_{\xi} \phi-\bar{\Gamma} \frac{(\bar{R}+\xi \bar{\delta}-\bar{\delta})}{\bar{\delta}} \frac{\partial \phi}{\partial \xi}\right]$

$$
+\frac{1}{\bar{\delta}(\bar{R}+\xi \bar{\delta}-\bar{\delta})} \frac{\partial}{\partial \chi}\left[\bar{\delta}(\bar{R}+\xi \bar{\delta}-\bar{\delta}) u_{\chi} \phi\right]=\bar{S}^{\bar{\phi}}+\nabla \Pi^{\bar{\phi}}+C^{\bar{\phi}}
$$

where for

$$
\begin{gathered}
\xi \text {-momentum: } \quad \bar{\phi}=u_{\xi}, \bar{S}^{\bar{\phi}}=\frac{u_{\theta}^{2}}{(\bar{R}+\xi \bar{\delta}-\bar{\delta})}-\frac{1}{\operatorname{Re}} \frac{u_{\xi}}{(\bar{R}+\xi \bar{\delta}-\bar{\delta})^{2}} \\
+\frac{1}{\operatorname{Re}} \frac{\left[\bar{\delta}^{\prime}(1-\xi)-\bar{R}^{\prime}\right] u_{x}}{(\bar{R}+\xi \bar{\delta}-\bar{\delta})^{2}}+\left(2 \Omega u_{\theta}+\Omega^{2} \bar{d}\right)\left\{\sin \alpha+\left[\bar{\delta}^{\prime}(1-\xi)-\bar{R}^{\prime}\right] \cos \alpha\right\} \\
+\Omega^{2}(\bar{R}+\xi \bar{\delta}-\bar{\delta}) \sin \alpha, \\
\nabla \Pi^{\bar{\phi}}=-\frac{1}{\bar{\delta}}\left\{1-\left[\bar{\delta}^{\prime}(1-\xi)-\bar{R}^{\prime}\right]^{2}\right\} \frac{\partial \Pi}{\partial \xi}+\left[\bar{\delta}^{\prime}(1-\xi)-\bar{R}^{\prime}\right] \frac{\partial \Pi}{\partial \chi}, \text { and } \bar{\Gamma}=\frac{1}{\operatorname{Re}},
\end{gathered}
$$




$$
\begin{aligned}
& \theta \text {-momentum: } \bar{\phi}=u_{\theta}, \bar{S}^{\bar{\phi}}=-\frac{u_{\xi} u_{\theta}}{(\bar{R}+\xi \bar{\delta}-\bar{\delta})}+\frac{\left[\bar{\delta}^{\prime}(1-\xi)-\bar{R}^{\prime}\right] u_{x} u_{\theta}}{(\bar{R}+\xi \bar{\delta}-\bar{\delta})} \\
& -\frac{1}{\operatorname{Re}} \frac{u_{\theta}}{(\bar{R}+\xi \bar{\delta}-\bar{\delta})^{2}}-2 \Omega u_{\xi} \sin \alpha+2 \Omega\left[\bar{\delta}^{\prime}(1-\xi)-\bar{R}^{\prime}\right] u_{x} \sin \alpha-2 \Omega u_{x} \cos \alpha \\
& \text { x-momentum: } \quad \nabla \Pi^{\bar{\phi}}=0 \text {, and } \bar{\Gamma}=\frac{1}{\operatorname{Re}}, \\
& \qquad \bar{\phi}=u_{x}, \bar{S}^{\bar{\phi}}=\Omega^{2} \bar{d} \cos \alpha+2 \Omega u_{\theta} \cos \alpha \\
& \quad \nabla \Pi^{\bar{\phi}}=-\frac{\partial \Pi}{\partial \chi}-\frac{\left[\bar{\delta}^{\prime}(1-\xi)-\bar{R}^{\prime}\right]}{\bar{\delta}} \frac{\partial \Pi}{\partial \xi} \text {, and } \bar{\Gamma}=\frac{1}{\operatorname{Re}}, \\
& \bar{\phi}=\Lambda, \bar{S}^{\bar{\phi}}=0, \nabla \Pi^{\bar{\phi}}=0 \text {, and } \bar{\Gamma}=\frac{1}{P e} \text {. }
\end{aligned}
$$

By using Eqs. (3.25) and (3.26), the following term appears as a source term in the $\xi$ momentum equation:

$$
C^{u_{\xi}}=u_{x}^{2}\left[\bar{\delta}^{\prime \prime}(1-\xi)-\bar{R}^{\prime \prime}\right]-\frac{\bar{\delta}^{\prime}}{\bar{\delta}} u_{\xi} u_{x}+\frac{2 \bar{\delta}^{\prime}}{\bar{\delta}^{2} \operatorname{Re}} \frac{\partial u_{x}}{\partial \xi}+\frac{\bar{\delta}^{\prime}}{\bar{\delta} \operatorname{Re}} \frac{u_{x}}{(\bar{R}+\xi \bar{\delta}-\bar{\delta})},
$$

and for the $\theta$-momentum, x-momentum, and energy equations $C^{\bar{\phi}}=0$.

The dimensionless boundary conditions are the no-slip conditions $u_{x}=u_{\xi}=u_{\theta}=0$ at the wall of the heat pipe $(\xi=1)$ and at the end caps ( $\chi=0$ and $\left.\chi=L_{\text {tot }} / R_{0}\right)$. The heat flux to the wall of the heat pipe, in dimensionless form, is given by

$$
\bar{q}^{\prime \prime}=\frac{N u}{\sqrt{1+\bar{R}^{\prime 2}}}\left[-\frac{\left(1+\bar{R}^{\prime 2}\right)}{\bar{\delta}} \frac{\partial \Lambda}{\partial \xi}+\bar{R}^{\prime} \frac{\partial \Lambda}{\partial \chi}\right]_{\xi=1},
$$


where the dimensionless heat flux is defined as $\vec{q}^{\prime \prime}=q^{\prime \prime} R_{0}^{2} / Q$. The insulated conditions at the end caps are expressed as $\partial \Lambda /\left.\partial \chi\right|_{\chi=0}=\partial \Lambda /\left.\partial \chi\right|_{\chi=L_{\text {tot }} / R_{0}}=0$. The conditions at $\xi=0$ become (Sim et al. 2004)

$$
\begin{gathered}
\Lambda=T_{s} / T_{c h} \\
u_{\xi}=-N u\left\{\frac{\left[1+\left(\overline{\delta^{\prime}}-\bar{R}^{\prime}\right)^{2}\right]}{\bar{\delta}} \frac{\partial \Lambda}{\partial \xi}+\left(\overline{\delta^{\prime}}-\bar{R}^{\prime}\right) \frac{\partial \Lambda}{\partial \chi}\right\} \\
A_{\xi \xi} \frac{\partial u_{\xi}}{\partial \xi}+A_{\xi \chi} \frac{\partial u_{\xi}}{\partial \chi}=B_{n} .
\end{gathered}
$$

For wicked operation the no-slip conditions are $u_{\theta}=u_{x}=0$ at $\xi=0$, and for non-wicked operation the tangential stress balances are

$$
\begin{gathered}
A_{\theta \xi} \frac{\partial u_{\theta}}{\partial \xi}+A_{\theta \chi} \frac{\partial u_{\theta}}{\partial \chi}=B_{\theta} \\
B_{\xi \xi} \frac{\partial u_{\xi}}{\partial \xi}+B_{\xi \chi} \frac{\partial u_{\xi}}{\partial \chi}+B_{\chi \xi} \frac{\partial u_{\chi}}{\partial \xi}+B_{\chi \chi} \frac{\partial u_{\chi}}{\partial \chi}=B_{t}
\end{gathered}
$$

The coefficients in the normal stress jump condition of Eq. (3.37) are:

$$
\begin{aligned}
& A_{\xi \xi}=2\left[1+\left(\overline{\delta^{\prime}}-\bar{R}^{\prime}\right)^{2}\right] /(\bar{\delta} \mathrm{Re}), A_{\xi \chi}=2\left(\overline{\delta^{\prime}}-\bar{R}^{\prime}\right) / \mathrm{Re}, \text { and } \\
& B_{n}=\left(1-\rho / \rho_{v}\right)\left[1+\left(\overline{\delta^{\prime}}-\bar{R}^{\prime}\right)^{2}\right] u_{\xi}^{2}+2 \frac{\left(\overline{\delta^{\prime}}-\bar{R}^{\prime}\right)\left(\bar{\delta}^{\prime \prime}-\bar{R}^{\prime \prime}\right)}{\operatorname{Re}} u_{x}-f \frac{\left(\overline{\delta^{\prime}}-\bar{R}^{\prime}\right)}{4} \frac{\rho_{v}}{\rho}\left\langle u_{v}\right\rangle^{2} \\
& +\left[1+\left(\bar{\delta}^{\prime}-\bar{R}^{\prime}\right)^{2}\right]\left(\Pi-\Pi_{v}\right)+\frac{2 H}{C a}\left[1+\left(\overline{\delta^{\prime}}-\bar{R}^{\prime}\right)^{2}\right]^{2},
\end{aligned}
$$

where the Capillary number is defined as $C a=\rho \mathrm{v}_{\text {char }}^{2} R_{0} / \sigma$. The coefficients in the tangential stress balance of Eq. (3.38) are given as 


$$
A_{\theta \xi}=\frac{1}{\bar{\delta}}\left[1+\left(\bar{\delta}^{\prime}-\bar{R}^{\prime}\right)\right], A_{\theta \chi}=1 \text {, and } B_{\theta}=\frac{u_{\theta}}{(\bar{R}-\bar{\delta})},
$$

and the coefficients in Eq. (3.39) are given as

$$
\begin{gathered}
B_{\xi \xi}=\frac{\left(\bar{\delta}^{\prime}-\bar{R}^{\prime}\right)}{\bar{\delta} \operatorname{Re}}, B_{\xi \chi}=\frac{\left[\left(\bar{\delta}^{\prime}-\bar{R}^{\prime}\right)^{2}-1\right]}{\left[1+\left(\bar{R}^{\prime}-\bar{\delta}^{\prime}\right)^{2}\right] \operatorname{Re}}, B_{\chi \xi}=-\frac{\left[1+\left(\overline{\delta^{\prime}}-\bar{R}^{\prime}\right)^{2}\right]}{\bar{\delta} \operatorname{Re}}, \\
B_{\chi \chi}=-\frac{\left(\bar{\delta}^{\prime}-\bar{R}^{\prime}\right)}{\operatorname{Re}}, \text { and } B_{t}=\frac{\left[\left(\bar{\delta}^{\prime}-\bar{R}^{\prime}\right)^{2}-1\right]}{\left[1+\left(\bar{\delta}^{\prime}-\bar{R}^{\prime}\right)^{2}\right]} \frac{\rho_{v}}{\rho} \frac{f}{8}\left\langle u_{v}\right\rangle^{2} .
\end{gathered}
$$

In this set of non-dimensional equations and boundary conditions, a number of dimensionless parameters appear. The definitions of these parameters are summarized in Table 3.1. The variables in the left-hand column of Table 3.1 were first defined and the dimensionless groups in the right-hand column are a result of applying those definitions to the governing equations and boundary conditions. The dimensionless velocity and temperature are defined in terms of a general characteristic velocity and temperature, $\mathrm{v}_{c h}$ and $T_{c h}$. These characteristic values are retained throughout the formulation and appear in the resulting dimensionless groups. Formulating the model in this way allows the equations and boundary conditions to be easily modified if different characteristic scales are required. For the heat pipe with constant heat flux boundary conditions, the characteristic velocity and temperature, $\mathrm{v}_{c h}=Q /\left(\rho h_{f g} R_{0}^{2}\right)$ and $T_{c h}=Q /\left(k R_{0}\right)$, are chosen in terms of the heat load carried by the heat pipe.

\subsubsection{NAtural Convection in the Evaporator}

To complete the model, a Nusselt number correlation is needed to evaluate the diffusion term in the energy equation. For the stationary heat pipe with the annular gap 
Table 3.1. Dimensionless parameters in the numerical model.

\begin{tabular}{|l|ll|}
\hline Dimensionless Variables & \multicolumn{2}{l|}{ Dimensionless Groups } \\
\hline Dependent Variables: & Reynolds & $\mathrm{Re}=\rho \mathrm{v}_{c h} R_{0} / \mu$ \\
Velocity $\quad \mathbf{u}=\mathbf{v} / \mathrm{v}_{c h}$ & Péclet & $P e=\rho C_{p} \mathrm{v}_{c h} R_{0} / k$ \\
Temperature $\quad \Lambda=T / T_{c h}$ & Capillary & $C a=\rho \mathrm{v}_{c h}^{2} R_{0} / \sigma$ \\
Independent Variables: & Rotation & $\Omega=\omega R_{0} / \mathrm{v}_{c h}$ \\
Axial Coordinate $\quad \chi=x / R_{0}$ & Flow Rate & $\bar{m}=\dot{m} /\left(\rho \mathrm{v}_{c h} R_{0}^{2}\right)$ \\
Radial Coordinate $\quad \xi=(r-R+\delta) / \delta$ & Pressure & $\Pi=p /\left(\rho \mathrm{v}_{c h}^{2}\right)$ \\
\hline
\end{tabular}

wick, heat transfer across the liquid film is treated as pure conduction by letting $N u=1$. Song et al. have shown that for an axially rotating heat pipe, natural convection in the liquid film of the evaporator section has a significant effect on the overall thermal resistance of the heat pipe (Song et al. 2003; Song et al. 2004). Song et al. use the empirical correlation developed by Körner for natural convection in an evaporating liquid film lining a rotating boiler, $N u=0.133 R a^{0.375}$, where the Rayleigh number is based on the layer thickness (Körner 1970). After reproducing the results of Song et al., using the code developed here, it was observed that Rayleigh numbers were typically $R a<10^{4}$ in the evaporator sections of the heat pipes studied. The experimental work performed by Körner, however, was restricted to the fully turbulent regime $\left(10^{7}<R a<10^{10}\right)$. For this reason, steady laminar natural convection in an evaporating liquid film, restricted to the low ( $R a<10^{4}$ ) Rayleigh number regime, was modeled by Jankowski et al. (Jankowski et al. 2006a) (see also Appendix B). In the low Rayleigh number regime, the correlation of Körner predicts considerably higher ( $80 \%$ at $R a=10^{3}$ to $40 \%$ at $R a=10^{4}$ ) heat transfer coefficients than that of Jankowski et al. The heat pipe design code developed here includes both the correlation developed by Körner (given above) and that of Jankowski et al., 


$$
N u=1+2.2(1-1600 / R a)^{*}+\left[(R a / 5830)^{1 / 3}-1\right]^{*},
$$

where the notion []$^{*}$ indicates that if a negative quantity is calculated in the bracketed term, the bracketed term is taken as zero.

\subsubsection{VAPOR FLOW MOdEL}

The liquid flow is coupled to the vapor flow through the specification of the interface boundary conditions. In the boundary conditions at the liquid-vapor interface, the average axial velocity of the vapor, the friction factor, the pressure of the vapor at the interface, and the temperature of the vapor at the interface must be specified. A onedimensional vapor flow model, based on the flow formulations used in the steady state heat pipe analysis code HTPIPE (Woloshun et al. 1988), is used here.

For the one-dimensional vapor flow, the average axial velocity of the vapor can be related to the mass flow rate at a given axial location by

$$
\left\langle\mathrm{v}_{v}\right\rangle=-\frac{\dot{m}}{\rho_{v} \pi(R-\delta)^{2}} .
$$

The pressure drop in the evaporator, adiabatic section, and condenser are approximated by one-dimensional formulations. Following Busse (Busse 1967), the vapor flow in the evaporator section is modeled as laminar and incompressible. Busse's expression for the pressure drop includes a viscous term and a convective term. Here, a body force term is also included to account for the centrifugal force acting on the vapor. In terms of dimensional variables, the pressure drop in the evaporator section is then 


$$
\begin{aligned}
\left.\frac{\partial p_{v}}{\partial x}\right|_{R-\delta}=\frac{\mathrm{d} p_{v}}{\mathrm{~d} x}= & -\frac{8 \mu_{v}\left\langle\mathrm{v}_{v}\right\rangle}{(R-\delta)^{2}}(1+0.667 a) \\
& -\frac{4}{3} \rho_{v} \frac{\mathrm{d}}{\mathrm{d} x}\left[\overline{\mathrm{v}}_{v}^{2}\left(1-a / 6+2 a^{2} / 45\right)\right]+\rho_{v} \omega^{2} d \cos \alpha, \\
& a=\frac{15}{22}\left[5+\frac{18}{\mathrm{Re}_{r}}-\sqrt{\left(5+\frac{18}{\mathrm{Re}_{r}}\right)^{2}-\frac{44}{5}}\right] .
\end{aligned}
$$

The radial Reynolds number in the evaporator is defined as

$$
\operatorname{Re}_{r}=\frac{Q}{2 \pi h_{f g} L_{e} \mu_{v}} .
$$

The vapor flow in the adiabatic section is treated as a one-dimensional, fully developed, incompressible flow. Depending on the flow velocity, the flow is treated as either laminar or turbulent. The pressure drop in the vapor phase for the adiabatic section is given by

$$
\left.\frac{\partial p_{v}}{\partial x}\right|_{R-\delta}=\frac{\mathrm{d} p_{v}}{\mathrm{~d} x}=\frac{\rho_{v} f\left\langle\mathrm{v}_{v}\right\rangle^{2}}{4(R-\delta)}+\rho_{v} \omega^{2} d \cos \alpha,
$$

where the friction factor is (Fox and McDonald 1992)

$$
f=\left\{\begin{array}{ll}
64 / \mathrm{Re}_{v} & \mathrm{Re}_{v}<2300 \\
0.25\left[\log _{10}\left(\frac{5.74}{\operatorname{Re}_{v}^{0.9}}\right)\right]^{-2} & \mathrm{Re}_{v} \geq 2300
\end{array},\right.
$$

and the axial flow Reynolds number is

$$
\operatorname{Re}_{v}=\frac{2 \rho_{v}\left\langle\mathrm{v}_{v}\right\rangle(R-\delta)}{\mu_{v}} .
$$

The vapor flow in the condenser section is treated as an incompressible flow that can be either laminar or turbulent. The pressure drop term in Eq. (3.48) is modified by a 
pressure recovery (or inertial/convective) term suggested by Kemme (Woloshun et al. 1988). The pressure drop in the condenser is calculated from

$$
\left.\frac{\partial p_{v}}{\partial x}\right|_{R-\delta}=\frac{\mathrm{d} p_{v}}{\mathrm{~d} x}=\frac{\mathrm{d} p_{c}}{\mathrm{~d} x}+\frac{\rho_{v} f\left\langle\mathrm{v}_{\mathrm{v}}\right\rangle^{2}}{4(R-\delta)}+\rho_{v} \omega^{2} d \cos \alpha,
$$

where

$$
\frac{\mathrm{d} p_{c}}{\mathrm{~d} x}=\frac{\mathrm{Re}_{r}+2}{\left(2 L_{e}+4 L_{a}-1.23 \operatorname{Re}_{r} L_{c}\right)} \frac{Q^{2}}{\pi^{2} h_{f g}^{2} \rho_{v}(R-\delta)^{4}},
$$

and the radial Reynolds number in the condenser is given by

$$
\operatorname{Re}_{r}=-\frac{Q}{2 \pi h_{f g} L_{c} \mu_{v}} .
$$

The axial coordinate, pressure, and vapor velocity are made dimensionless using the expressions in Eq. (3.18). In terms of dimensionless variables, the expressions for the vapor phase pressure drop in each section of the heat pipe are given by

$$
\begin{aligned}
& \text { evaporator section: } \begin{aligned}
& \frac{\mathrm{d} \Pi_{v}}{\mathrm{~d} \chi}=-\frac{8 \mu_{v}\left\langle u_{v}\right\rangle}{\mu \operatorname{Re}(\bar{R}-\bar{\delta})^{2}}(1+0.667 a) \\
&-\frac{4}{3} \frac{\rho_{v}}{\rho} \frac{\mathrm{d}}{\mathrm{d} \chi}\left[\left\langle u_{v}\right\rangle^{2}\left(1-a / 6+2 a^{2} / 45\right)\right]+\frac{\rho_{v}}{\rho} \Omega^{2} \frac{d}{R_{0}} \cos \alpha \\
& \text { adiabatic section: } \quad \frac{\mathrm{d} \Pi_{v}}{\mathrm{~d} \chi}=f \frac{\rho_{v}}{\rho} \frac{\left\langle u_{v}\right\rangle^{2}}{4(\bar{R}-\bar{\delta})}+\frac{\rho_{v}}{\rho} \Omega^{2} \frac{d}{R_{0}} \cos \alpha
\end{aligned}
\end{aligned}
$$

condenser section: $\frac{\mathrm{d} \Pi_{v}}{\mathrm{~d} \chi}=\frac{\operatorname{Re}_{r}+2}{\left(2 L_{e}+4 L_{a}-1.23 \operatorname{Re}_{r} L_{c}\right) / R_{0}} \frac{\rho_{l}}{\rho} \frac{1}{\pi^{2}(\bar{R}-\bar{\delta})^{4}}$

$$
+f \frac{\rho_{v}}{\rho} \frac{\left\langle u_{v}\right\rangle^{2}}{4(\bar{R}-\bar{\delta})^{5}}+\frac{\rho_{v}}{\rho} \Omega^{2} \frac{d}{R_{0}} \cos \alpha,
$$


Once the pressure of the vapor has been calculated, the saturation temperature at the liquid-vapor interface is determined using the Clapeyron equation. In terms of dimensionless variables, the saturation temperature is given by

$$
\frac{T_{s}}{T_{c h}}(\chi+\Delta \chi)=\frac{T_{s}}{T_{c h}}(\chi) \exp \left(\frac{v_{f g} p_{c h}}{h_{f g}} \frac{\mathrm{d} \Pi_{v}}{\mathrm{~d} \chi} \Delta \chi\right) .
$$

\subsubsection{Numerical Solution}

Equations (3.27)-(3.32) subject to the dimensionless boundary conditions are solved numerically. The 'parabolic flow' assumption is made here, which allows for a spacemarching procedure to be used in the solution of the governing equations. Accordingly, the solution begins at the condenser end-cap and a series of one-dimensional problems are solved while marching in the $\chi$-direction toward the evaporator end-cap. The parabolic flow assumption implies that (a) there is no reverse flow in the predominant flow direction (in this case the $\chi$-direction), (b) diffusion of momentum and energy in the main flow direction are negligible, and (c) that the downstream pressure field has little influence on the upstream flow (Patankar and Spalding 1972). Throughout the formulation, the diffusion terms in the predominant flow direction have been ignored as required by assumption (b) listed above. To use the space-marching procedure, the pressure gradient in the predominant flow direction, $\partial \Pi / \partial \chi$, must be approximated by a pressure gradient averaged over the cross-section in the $\xi-\theta$ plane, $\partial \bar{\Pi} / \partial \chi$. This averaged pressure gradient is determined in the solution procedure so that a global mass balance is satisfied at each $\chi$-location. In terms of dimensionless variables, the mass flow rate at each $\chi$-location is given by 


$$
\frac{\bar{m}}{2 \pi \bar{\delta}}=\int_{\xi=0}^{\xi=1}(\bar{R}+\xi \bar{\delta}-\bar{\delta}) u_{x} \mathrm{~d} \xi,
$$

where the dimensionless mass flow rate is $\bar{m}=\dot{m} /\left(\rho \mathrm{v}_{c h} R_{0}^{2}\right)$. The mass flow rate can be evaluated by considering a global mass balance applied to a volume element of length $\Delta \chi$. Liquid is entering the volume element with a flow rate of $\bar{m}_{i-1}$ at location $\chi_{i-1}$ and leaving at $\chi_{i}$ with a flow rate of $\bar{m}_{i}$. The difference between these flow rates is given by the flow rate crossing the free-surface of the liquid (by evaporation or condensation). In terms of dimensionless variables, the global mass balance is

$$
\begin{aligned}
\bar{m}_{i}=\bar{m}_{i-1}+\int_{\chi}^{\chi+\Delta \chi}(\bar{R}-\bar{\delta})(\mathbf{u} \bullet \mathbf{n}) \mathrm{d} \chi & \\
& =\bar{m}_{i-1}+2 \pi \int_{\chi}^{\chi+\Delta \chi}(\bar{R}-\bar{\delta})\left[1-\left(\bar{\delta}^{\prime}-\bar{R}^{\prime}\right)^{2}\right]^{-1 / 2} u_{\xi} \mathrm{d} \chi .
\end{aligned}
$$

Equation (3.59) is used to evaluate the left-hand side of Eq. (3.58) at each $\chi$-location, and the discrete form of Eq. (3.58) is used to obtain an approximation for $\partial \bar{\Pi} / \partial \chi$. Details of this procedure can be found in the literature (Patankar and Spalding 1972; Prantap and Spalding 1976) and are summarized in Appendix A.

The equations, subject to the parabolic flow assumption, are discretized on a staggered grid using a control volume method. Throughout the simulations, uniform grids in both the $\chi$ and $\xi$-directions are used. False boundary nodes located at distances $\Delta \xi / 2$ beyond the horizontal boundaries of the solution domain are used to specify the values of the boundary conditions at the wall of the heat pipe and at the liquid-vapor interface. Throughout the discretization, pressure, temperature, $u_{x}$ and $u_{\theta}$ are stored at the main grid nodes, while $u_{\xi}$ is stored on the horizontal face of the control volume 
surrounding each main grid node. In the cross-stream direction (the $\xi$-direction), second-order centered-differences are used to approximate the diffusion terms, and the QUICK method (Leonard 1979) is used for the convective terms. First-order backward differences are used to approximate the convective terms in the $\chi$-direction. Derivatives appearing in the boundary conditions at the liquid-vapor interface are discretized using second-order finite-differences, and the boundary condition at the insulated condenser end-cap is implemented using a first-order forward difference formula. This implementation of the parabolic space-marching procedure results in a mixed-order discretization. By using a second-order discretization in the radial direction and a firstorder discretization in the axial direction, the overall order is expected to be between first and second order.

At each $\chi$-location, the discrete equations are solved using the SIMPLER method (Patankar 1980). At the liquid-vapor interface, the temperature specification [Eq. (3.35)] is applied as a boundary condition to the energy equation and the kinematic condition [Eq. (3.36)] is applied to the $\eta$-momentum equation. In wicked operation, the no-slip conditions are applied to the $\theta$ and $x$-momentum equations, and in non-wicked operation the discrete forms of the shear stress balances [Eqs. (3.38) and (3.39)] are applied to the $\theta$ and $x$-momentum equations, respectively.

In non-wicked operation, the normal stress jump condition is used to determine the liquid layer thickness. At each $\chi$-location, the layer thickness is determined iteratively. A guessed value of the layer thickness is first assumed (the value of the layer thickness from the previous $\chi$-location), and the governing equations are solved without imposing 
the normal stress balance of Eq. (3.37). After obtaining a solution, the residual of Eq. (3.37) is calculated,

$$
R_{n}=A_{\xi \xi} \frac{\partial u_{\xi}}{\partial \xi}+A_{\xi \chi} \frac{\partial u_{\xi}}{\partial \chi}-B_{n}
$$

If $\left|R_{n}\right|$ is greater than a specified tolerance, the layer thickness is updated with $\bar{\delta}=\bar{\delta}-\kappa R_{n}$, where $\kappa$ is a user-specified relaxation factor (Pacheco and Peck 2000).

In wicked operation, the liquid layer thickness is known (and is equal to the annular gap distance), so the normal stress jump condition [Eq. (3.37)] is used to determine the curvature of the liquid-vapor interface, $H$. If the curvature of the liquid-vapor interface is larger than the maximum curvature that can be supported by the porous wick structure, a dry-out of the heat pipe will occur.

The rotating heat pipe model has the ability to model a heat pipe with a given liquid charge. The liquid layer thickness at a particular $\chi$-location in the heat pipe is first guessed and further determined by a shooting method so that the mass of liquid in the heat pipe is equal to the fluid charge. The liquid mass is given by

$$
\rho V=2 \pi \rho R_{0}^{3} \int_{\xi=0}^{\xi=1} \int_{\chi=0}^{\chi=L_{\text {tot }} / R_{0}} \bar{\delta}(\bar{R}+\xi \bar{\delta}-\bar{\delta}) \mathrm{d} \chi \mathrm{d} \xi
$$

\subsubsection{Convergence CRiteria}

In the SIMPLER method, iterative convergence of the continuity, momentum, and energy equations is assumed after the residuals of each equation have been reduced three orders of magnitude from the residuals calculated with the initial guessed solution. The solution from the previous $\chi$-location is used as a guess at each location. The layer thickness at each $\chi$-location is updated until $R_{n} p_{c h}<10^{-6} \mathrm{~Pa}$, where $R_{n} p_{c h}$ is a 
dimensional residual of the normal stress condition with units of pressure. Similarly, the guessed layer thickness is updated until the mass of liquid in the heat pipe, as calculated by Eq. (3.61), is within $10^{-6} \mathrm{~kg}$ of the fluid charge.

\subsection{VERIFICATION AND VALIDATION}

To gain confidence in the results of the heat pipe code developed here, three separate verification and validation cases are explored. First, a straight non-rotating heat pipe with an annular gap wick structure is modeled and the results are compared to the steady state heat pipe analysis code HTPIPE (Woloshun et al. 1988). A cylindrical rotating heat pipe without a wick structure is then modeled, and the results are compared to the published results of Song et al. (Song et al. 2004). Finally, the liquid layer thickness in the condenser section of a rotating tapered heat pipe is calculated and the results of the present analysis are compared to those reported by Daniels and Al-Jumaily (Daniels and Al-Jumaily 1975). These validation cases are used to show that the model is able to simulate heat pipes with or without a wick structure. The comparisons will also show that the liquid distribution is predicted correctly for rotating heat pipes with or without a component of the centrifugal force acting directly in the liquid flow direction.

For the verification and validation studies, the stationary heat pipe with the annular gap wick and the rotating cylindrical heat pipe are straight heat pipes filled with saturated water at $373 \mathrm{~K}$. Both heat pipes have a constant inside radius of $0.97 \mathrm{~cm}$, and the lengths of the evaporator, adiabatic, and condenser sections are $L_{e}=12.1 \mathrm{~cm}, L_{a}=18.4 \mathrm{~cm}$, and $L_{c}=10.2 \mathrm{~cm}$. The dimensions of the heat pipe containers considered here are the same as those used in the numerical and experimental studies of the axially rotating heat pipe (without a wick) by Song et al. (Song et al. 2003; Song et al. 2004). For the stationary 
heat pipe with an annular gap wick, the thickness of the annulus is set to $\delta=0.029 \mathrm{~cm}$, and the maximum curvature that can be supported by the wick is $H_{\max }=2.26 \times 10^{6} \mathrm{~cm}^{-1}$. This maximum curvature corresponds to an effective pore radius of $r_{\text {eff }}=1 / H_{\max }=44 \mu m$, which is a typical value for a $200 \times 200$ mesh screen wick.

The rotating tapered condenser section has the same dimensions used in the analytical and experimental investigations by Daniels and Al-Jumaily (Daniels and Al-Jumaily 1975). The length of the condenser section is set to $L_{c}=15.2 \mathrm{~cm}$, the inner wall radius at the condenser end-cap is set to $R_{0}=1.5 \mathrm{~cm}$, and the condenser wall has a taper of $2^{\circ}$ from the end-cap to intersection with the adiabatic section. The heat pipe is filled with Freon 113.

\subsubsection{Numerical Verification}

For each of these reference cases, grid convergence studies are carried out, and the Grid Convergence Index (GCI) is used to measure the error in the numerical solution (Roache 1998). In the GCI method, a parameter of interest is calculated using grids of various size. The GCI, which represents an error band that can be applied to these calculated values, is then evaluated.

For the heat pipe with the annular gap wick structure, pressure distributions in the liquid and vapor phases will be compared to the results of HTPIPE. For this reason, the total pressure drop in the liquid phase is used as a parameter of interest. The total pressure drop is defined as $\Delta p=p(x=0, r=R-\delta)-p\left(x=L_{\text {tot }}, r=R-\delta\right)$. Similarly, for the rotating cylindrical heat pipe, the average temperature difference between the evaporator and condenser is chosen as a parameter of interest, since this temperature difference will be compared to the results of Song et al. The parameter of interest for the 
rotating tapered condenser section is the average temperature difference across the liquid film. The temperature difference across the condenser film will be compared to the results of Daniels and Al-Jumaily in the validation studies.

Throughout the grid convergence studies, the apparent order of the numerical method, $\gamma$, is evaluated, and a safety factor of 1.25 is applied to the GCI. Roache suggests that evaluating the GCI in this manner gives $95 \%$ certainty that the GCI will be larger than the actual relative error (Roache 2003).

Results of the grid convergence studies are shown in Table 3.2. For the stationary wicked heat pipe, the grid convergence studies were carried out for the heat pipe transferring a $1500 \mathrm{~W}$ heat load. The total pressure drop was calculated with $(\chi \mathrm{x} \xi)$ grid sizes of $102 \times 45,102 \times 60$, and $102 \times 80$ to assess the effect of varying the grid size in the radial direction. The results show that with grid refinement in the radial direction, the code is converging to a solution as a second order method, $\gamma \sim 2$. This behavior

Table 3.2. Results of the grid convergence study for (a) the non-rotating heat pipe, (b) the cylindrical rotating heat pipe, and (c) the tapered condenser section. (a) Stationary Heat Pipe with Annular Gap Wick

\begin{tabular}{|c|c|c|c|c|c|c|c|}
\hline \multicolumn{4}{|c|}{ Convergence in Radial Direction } & \multicolumn{4}{|c|}{ Convergence in Both Directions } \\
\hline & \multicolumn{3}{|c|}{ grid size (axial) x (radial) nodes } & & \multicolumn{3}{|c|}{ grid size (axial) x (radial) nodes } \\
\hline & $102 \times 45$ & $102 \times 60$ & $102 \times 80$ & & $78 \times 45$ & $86 \times 60$ & $102 \times 80$ \\
\hline$\overline{\Delta \mathrm{p}(\mathrm{Pa})}$ & $\overline{425.6}$ & 433.1 & 438.7 & $\overline{\Delta \mathrm{p}(\mathrm{Pa})}$ & 429.2 & 432.8 & 438.7 \\
\hline \multicolumn{4}{|c|}{$\begin{array}{l}(\mathbf{1 0 2 \times 4 5 )})>(\mathbf{1 0 2 \times 8 0}) \text { apparent order, } \gamma=\mathbf{2 . 0} \\
(\mathbf{1 0 2 \times 8 0 )} \text { GCI }=\mathbf{4 . 8} \% \\
(102 \times 60) \text { GCI }=6.4 \%\end{array}$} & \multicolumn{4}{|c|}{$\begin{array}{l}(\mathbf{7 8 \times 4 5})->(\mathbf{1 0 2} \times 80) \text { apparent } \text { order, } \gamma=\mathbf{1 . 3} \\
(\mathbf{1 0 2 \times 8 0 )} \mathbf{G C I}=\mathbf{4 . 9} \% \\
(86 \times 60) \text { GCI }=6.6 \%\end{array}$} \\
\hline
\end{tabular}

\begin{tabular}{|c|c|c|c|c|c|c|c|}
\hline \multicolumn{4}{|c|}{ (b) Rotating Cylindrical Heat Pipe } & \multicolumn{4}{|c|}{ (c) Rotating Tapered Condenser Section } \\
\hline & \multicolumn{3}{|c|}{ grid size (axial) x (radial) nodes } & & \multicolumn{3}{|c|}{ grid size (axial) x (radial) nodes } \\
\hline & $52 \times 5$ & $77 \times 10$ & $102 \times 22$ & & $25 \times 5$ & $37 \times 10$ & $50 \times 22$ \\
\hline$\Delta \mathrm{T}(\mathrm{K})$ & 46.1 & 39.7 & 37.5 & $\Delta \mathrm{T}(\mathrm{K})$ & 27.8 & 27.5 & 27.6 \\
\hline \multicolumn{4}{|c|}{$\begin{array}{l}(77 \times 10)->(102 \times 22) \text { apparent order, } \gamma=1.9 \\
(102 \times 22) \text { GCI }=4.3 \% \\
(77 \times 10) \text { GCI }=11.0 \%\end{array}$} & \multicolumn{4}{|c|}{$\begin{array}{l}(37 \times 10)->(50 \times 22) \text { apparent order, } \gamma=\mathbf{2 . 0} \\
(50 \times 22) \text { GCI }=\mathbf{0 . 2} \% \\
(37 \times 10) \text { GCI }=0.7 \%\end{array}$} \\
\hline
\end{tabular}


would be expected since a second-order discretization (in the radial direction) has been used throughout. Additionally, the GCI suggests that the error in calculating the pressure difference in the liquid is less than $5 \%$ with the $102 \times 80$ grid. This procedure was repeated to assess the effect of varying the grid size in the both the axial and radial directions by using grids of $78 \times 45,86 \times 60$, and $102 \times 80$. The results show the expected reduction in the apparent order of the method resulting from the use of a firstorder discretization in the axial direction. Additionally, the calculated GCI for the $102 \mathrm{x}$ 80 grid agrees with the GCI calculated for the convergence study in the radial direction only.

The axially rotating heat pipe was simulated for a rotation speed of $4000 \mathrm{rpm}$ and a heat transfer rate of $400 \mathrm{~W}$. The fluid fill was set to $6.3 \mathrm{~g}$ of water. The liquid layer thickness at the condenser end cap, $\bar{\delta}_{0}$, was determined in the shooting method so that the liquid mass, calculated with Eq. (3.61), was equal to the fluid fill. The grid resolutions used in calculating the GCI were $52 \times 5,77 \times 10$, and $102 \times 22$, and the average temperature difference ( $\Delta T$ ) between the wall of the evaporator and condenser sections was used as the parameter of interest. Table 3.2 shows that the expected error for the 102 $\mathrm{x} 22$ grid is less than $5 \%$.

The tapered condenser section was modeled with a uniform heat flux at the outer wall. For the grid convergence study, the condenser was rotating at $1200 \mathrm{rpm}$ and with a $400 \mathrm{~W}$ heat rejection rate. Grid resolutions of 25 x 5, 37 x 10, and 50 × 22 were used for comparing the average temperature difference across the condenser film. Table 3.2 shows that less than $1 \%$ error can be expected with the $50 \times 22$ grid. 


\subsubsection{VALIDATION}

The pressure distributions in the liquid and vapor for the stationary wicked heat pipe with a $1500 \mathrm{~W}$ heat load are compared to the results of HTPIPE in Fig. 3.2. The results indicate that the present analysis is accurately predicting the liquid and vapor phase pressure drops when the liquid fills the wick.

In Fig. 3.3 comparisons are made between the model results of Song et al. and the present analysis for the rotating wickless heat pipe filled with $6.3 \mathrm{~g}$ of water at three different rotation speeds (2000, 3000, and $4000 \mathrm{rpm}$ ) and for three different heat loads (200, 300, and $400 \mathrm{~W}$ ) with the error bars representing the $5 \%$ GCI. The results shown in Fig. 3.3 were generated by the code developed here using the Nusselt number correlation of Jankowski et al., Eq. (3.43), in the evaporator section. The model results agree with those of Song et al., except at the lowest rotation speed (2000 rpm), where the present analysis is predicting higher temperature differences than the previous model.

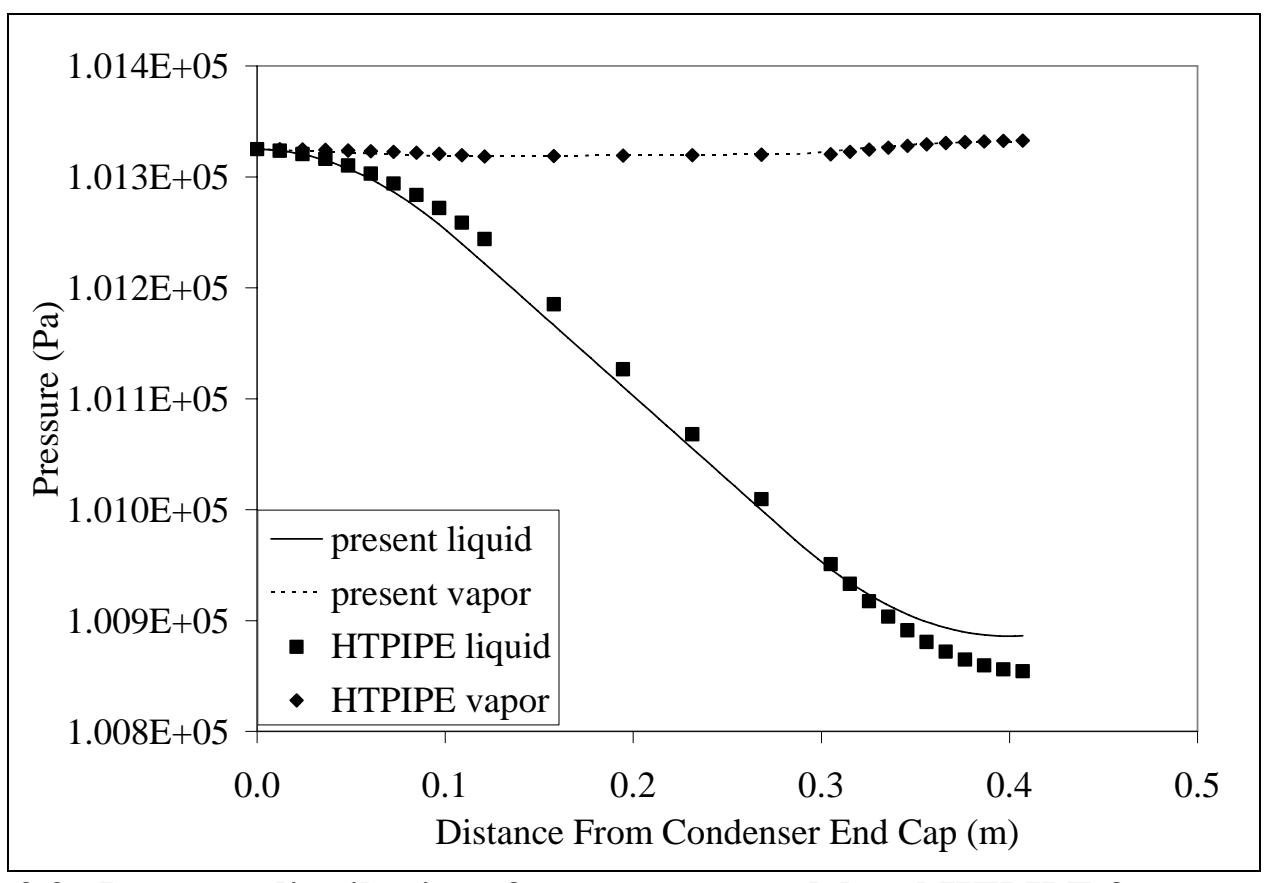

Figure 3.2. Pressure distributions from present model and HTPIPE for a stationary heat pipe. 


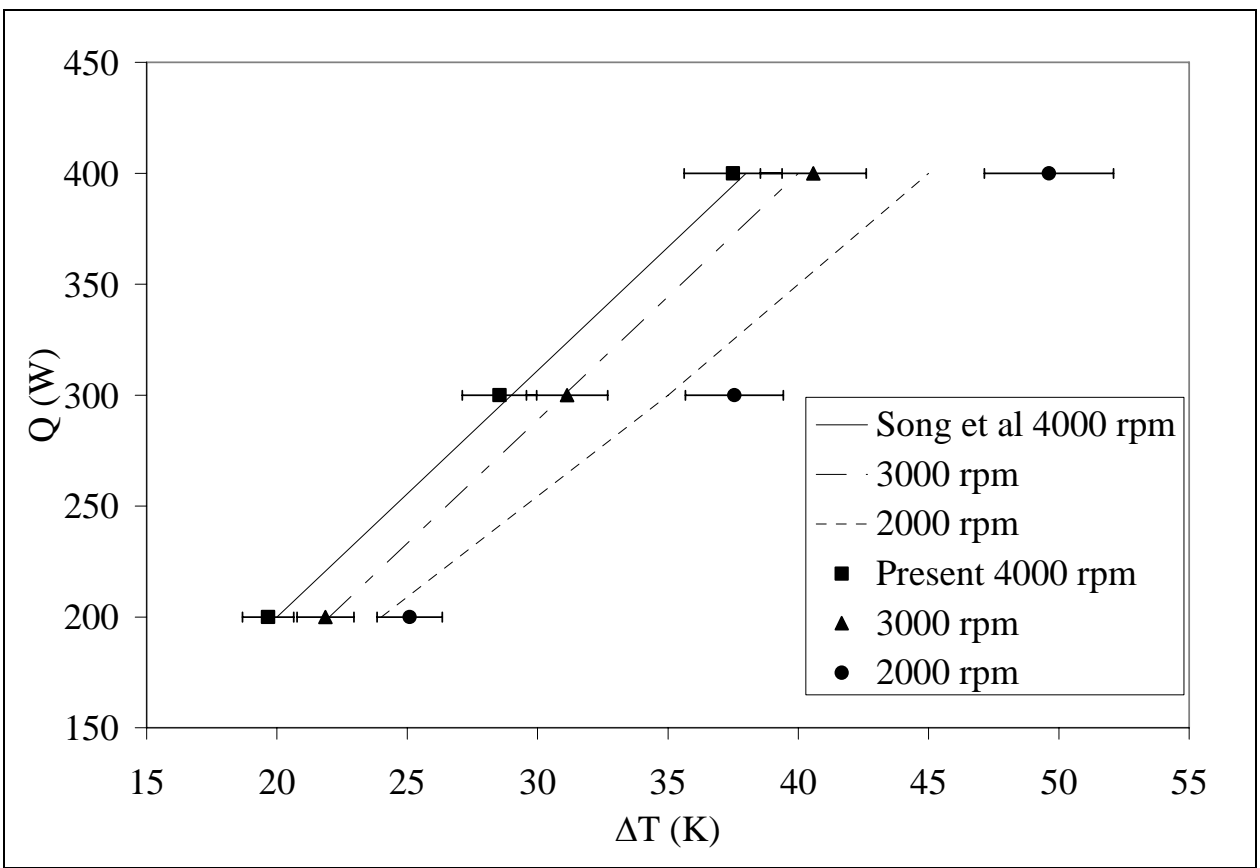

\section{Figure 3.3. Model results for an axially rotating heat pipe compared to the results of the model by Song et al.}

For the calculations at $2000 \mathrm{rpm}$, the Rayleigh number in the liquid film in the evaporator was lower than the critical value of 1600, representing the transition from pure conduction to the onset of convection in Eq. (3.43). For the $2000 \mathrm{rpm}$ cases, the correlation in Eq. (3.43) is producing $N u=1$, whereas the correlation of Körner, which is used in the model of Song et al., gives a Nusselt number near 2. Other than this explainable discrepancy at low speed, the present model does agree with the previous model of the rotating heat pipe within the bounds of the GCI.

In Fig. 3.4, the liquid layer thickness in the rotating tapered condenser section is shown. The calculated layer thickness from the present model and the work of Daniels and Al-Jumaily are compared for a heat load of $400 \mathrm{~W}$ and a rotation speed of $1200 \mathrm{rpm}$. The present analysis predicts a layer thickness that is $5 \%$ thinner than the analysis of Daniels and Al-Jumaily. The average temperature difference across the liquid film in the condenser as a function of heat load is shown in Fig. 3.5. The present model reproduces 
the general trends of increasing temperature difference with increasing heat load or decreasing rotation speed predicted in the analysis of Daniels and Al-Jumaily. The temperature differences predicted by the two analyses agree to within $20 \%$.

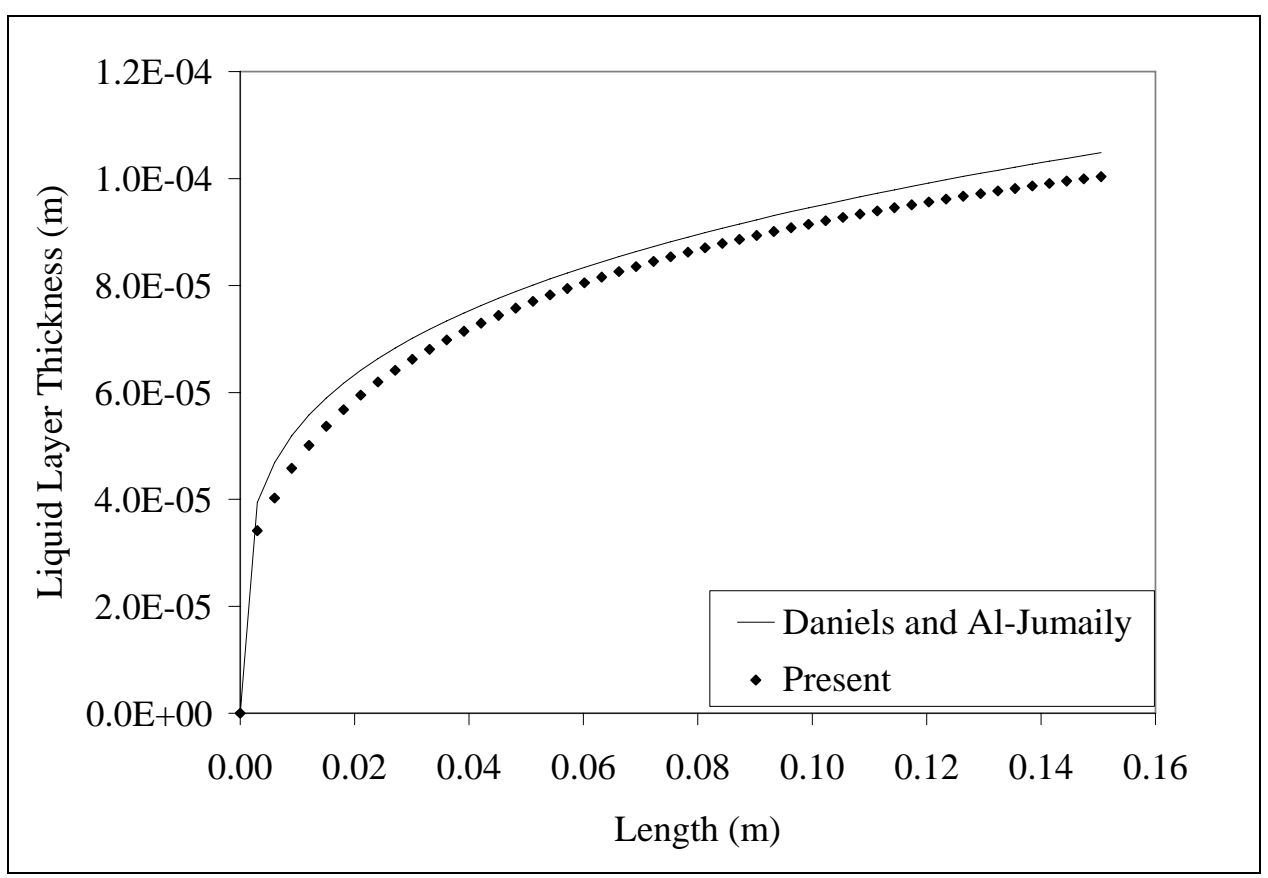

Figure 3.4. Liquid layer thickness in the tapered rotating condenser section.

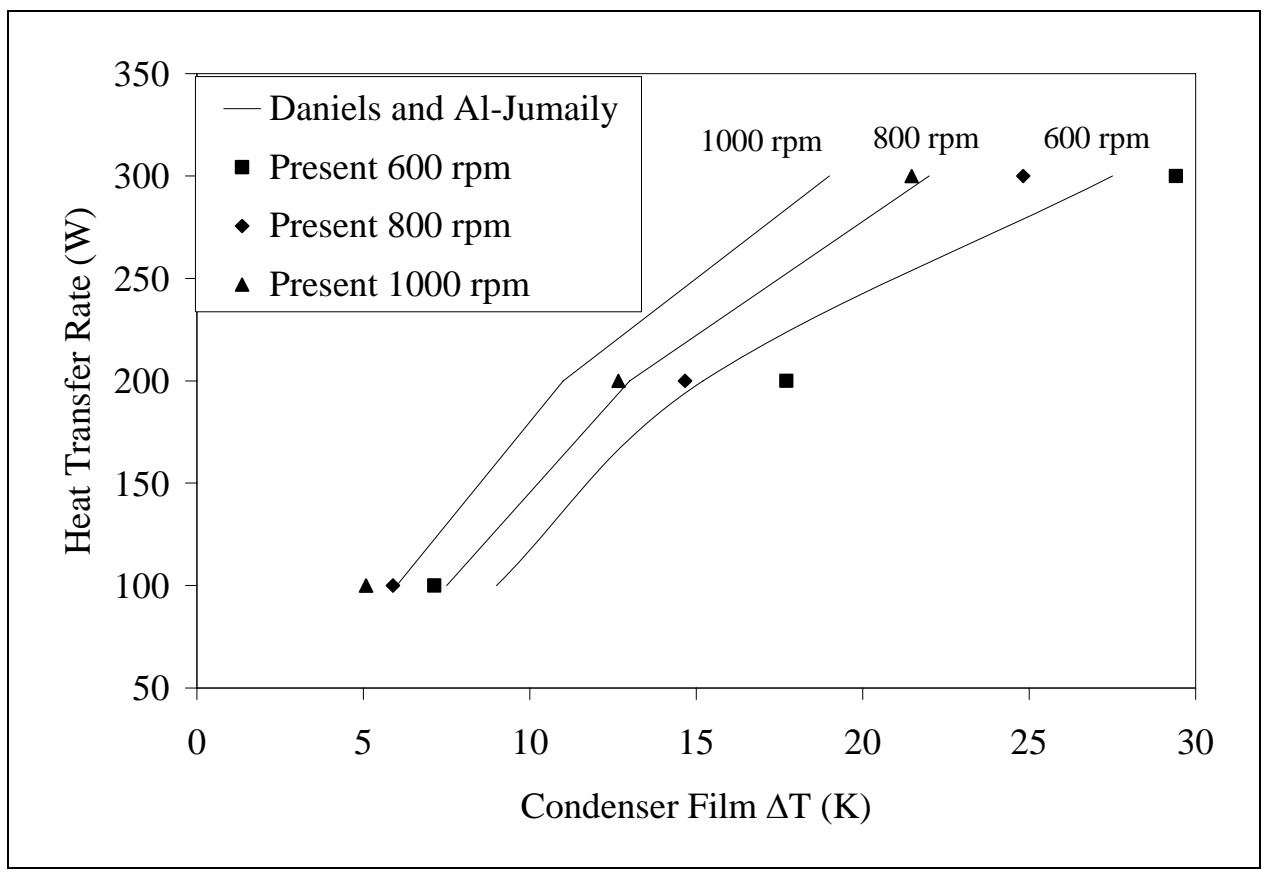

Figure 3.5. Temperature difference across the condenser film required to transfer heat in the rotating tapered condenser section. 
The marginal agreement observed in Fig. 3.5 may be attributed to the use of a constant heat flux boundary condition at the surface of the condenser in the present analysis, whereas Daniels and Al-Jumaily applied a constant surface temperature boundary condition. Additionally, in the analysis by Daniels and Al-Jumaily, the average radius of the tapered condenser section is used to evaluate the centrifugal force term in the momentum balance, whereas the present analysis uses the local tube radius. Previous studies have also shown that, as with the results in Fig. 3.4, using the average radius of the tapered condenser section as opposed to the local radius will over-predict the liquid layer thickness in the tapered condenser section (Li et al. 1993).

\subsection{Results for the Proposed Heat Pipe}

To illustrate the behavior of the proposed heat pipe, the model developed in this chapter is applied to a rotating heat pipe incorporating an S-shaped curve. The dimensions of the heat pipe modeled here are shown in Fig. 3.6. The heat pipe is made from a tube with a constant inside radius of $R=0.5 \mathrm{~cm}$, and the length of the evaporator, adiabatic, and condenser sections are $L_{e}=45.7 \mathrm{~cm}, L_{a}=63.5 \mathrm{~cm}$, and $L_{c}=30.5 \mathrm{~cm}$. The

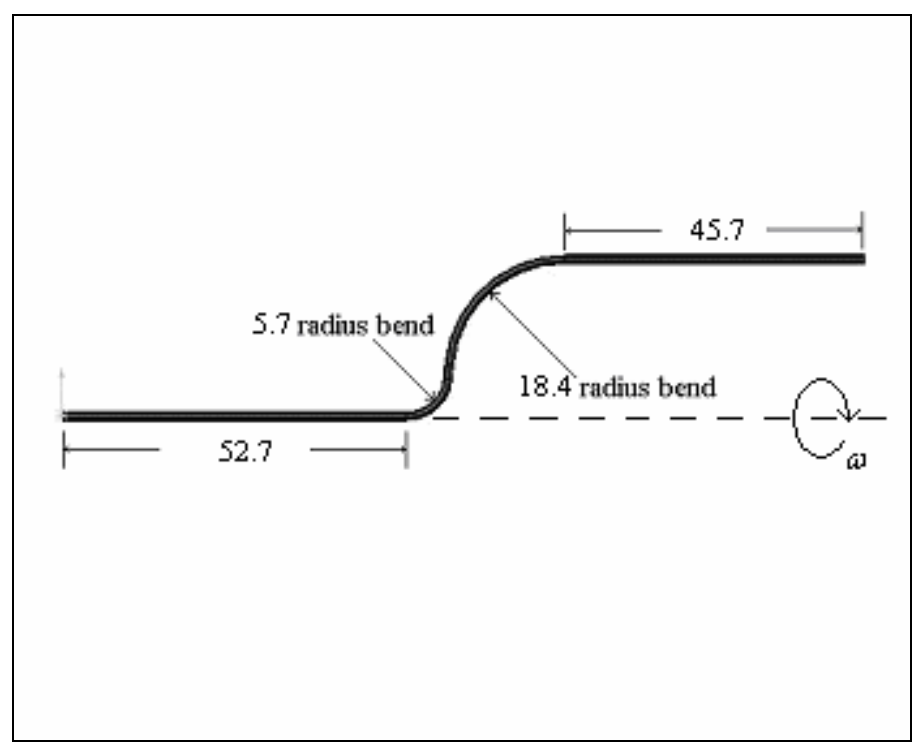

Figure 3.6. The rotating heat pipe modeled here. All dimensions are in centimeters. 
annular gap distance is set to $\delta=0.025 \mathrm{~cm}$, and the maximum curvature at the surface of the wick is $H_{\max }=2.26 \times 10^{6} \mathrm{~cm}^{-1}$. The S-shaped curve makes up part of the adiabatic section, with the maximum distance from the axis of rotation to the off-axis evaporator section of $d=24.1 \mathrm{~cm}$. The heat pipe is filled with methanol and fluid properties are evaluated at $333 \mathrm{~K}$.

Results are shown in Fig. 3.7 for the stationary (non-rotating) horizontal heat pipe with a heat load of $100 \mathrm{~W}$. The familiar pressure distribution in the stationary heat pipe is observed, with a wet-point near the condenser end-cap and the maximum pressure difference supported by the wick at the end of the evaporator. In Fig. 3.8, the curvature of the liquid-vapor interface at the surface of the wick is shown. The figure shows that, at the evaporator end, the wick is operating at $85 \%$ of the maximum capillary pressure that can be supported.

In Figs. 3.9 and 3.10, the pressure distribution and curvature at the liquid-vapor

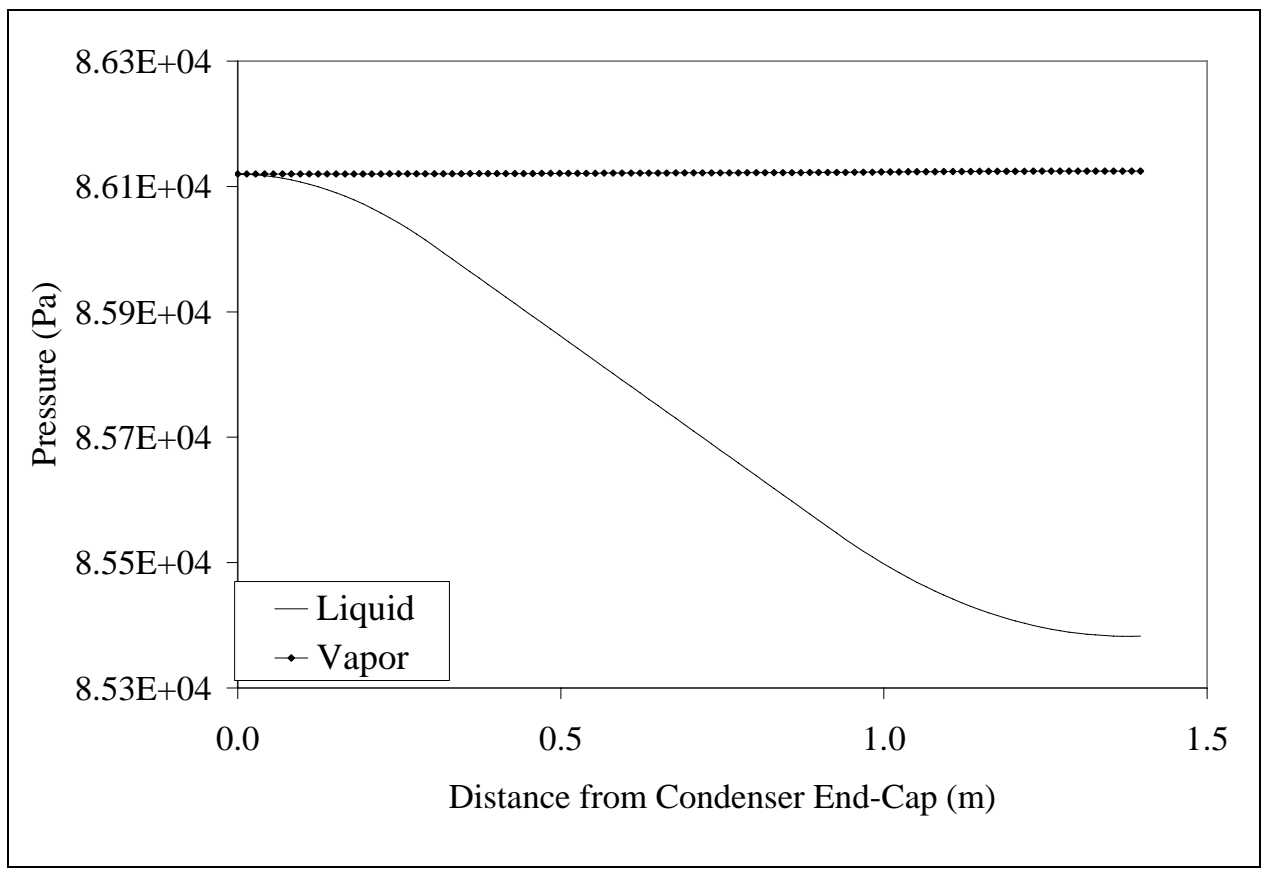

Figure 3.7. Pressure distributions for the bent heat pipe at 0 rpm. 


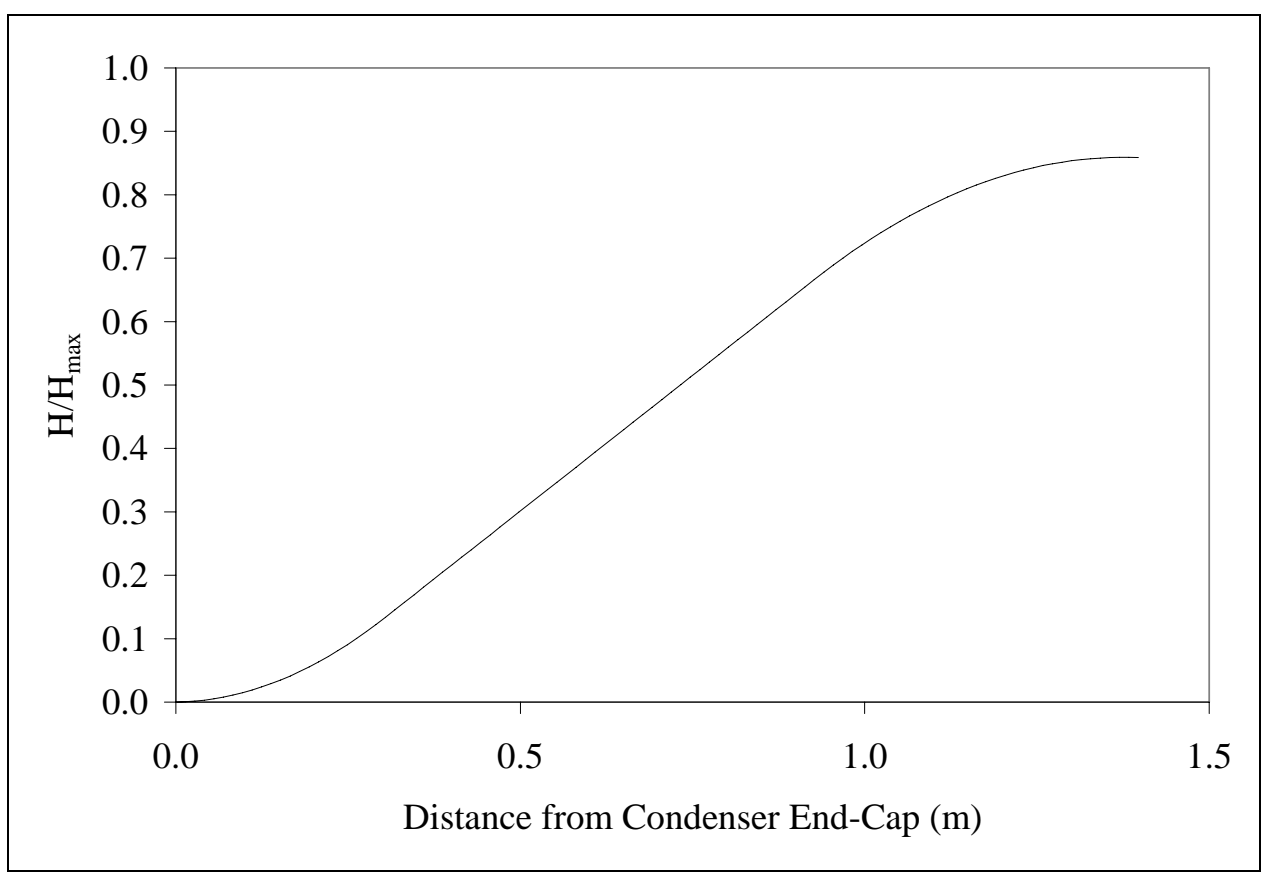

Figure 3.8. Curvature supported by the wick for the bent heat pipe at 0 rpm.

interface in the heat pipe rotating at $45 \mathrm{rpm}$ with a $100 \mathrm{~W}$ heat load are shown. The figures show the effect that the centrifugal force has on the pressure distributions in the liquid and vapor phases. A component of the centrifugal force acts directly in the predominant flow direction of both the liquid and vapor phases in the S-shaped curve. Because the density of the liquid phase is higher than the density of the vapor, the pressure rise in the liquid is higher than the pressure rise in the vapor, and the centrifugal force reduces the net pressure difference that must be supported by the wick. As the rotation speed is increased beyond $45 \mathrm{rpm}$, the pressure difference supported by the wick in the S-shaped curve will decrease to a point where the pressure difference goes to zero (a second wet-point). Further increases in the rotation speed can only be accommodated in the incompressible liquid flow by acceleration. The accelerating liquid flow will cause the liquid layer to recede from the wick and form a variable thickness film along the wall of the heat pipe. 


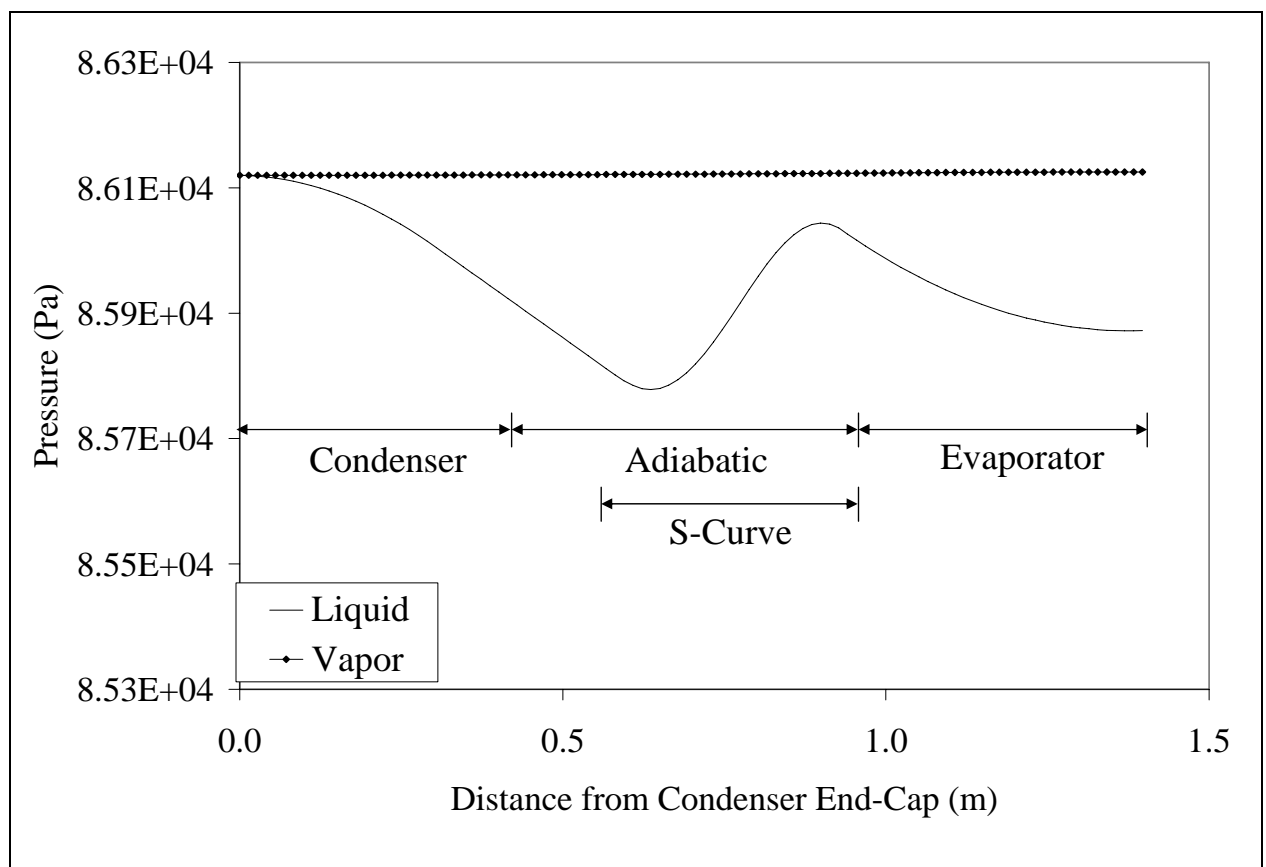

Figure 3.9. Pressure distributions for the bent heat pipe at 45 rpm.

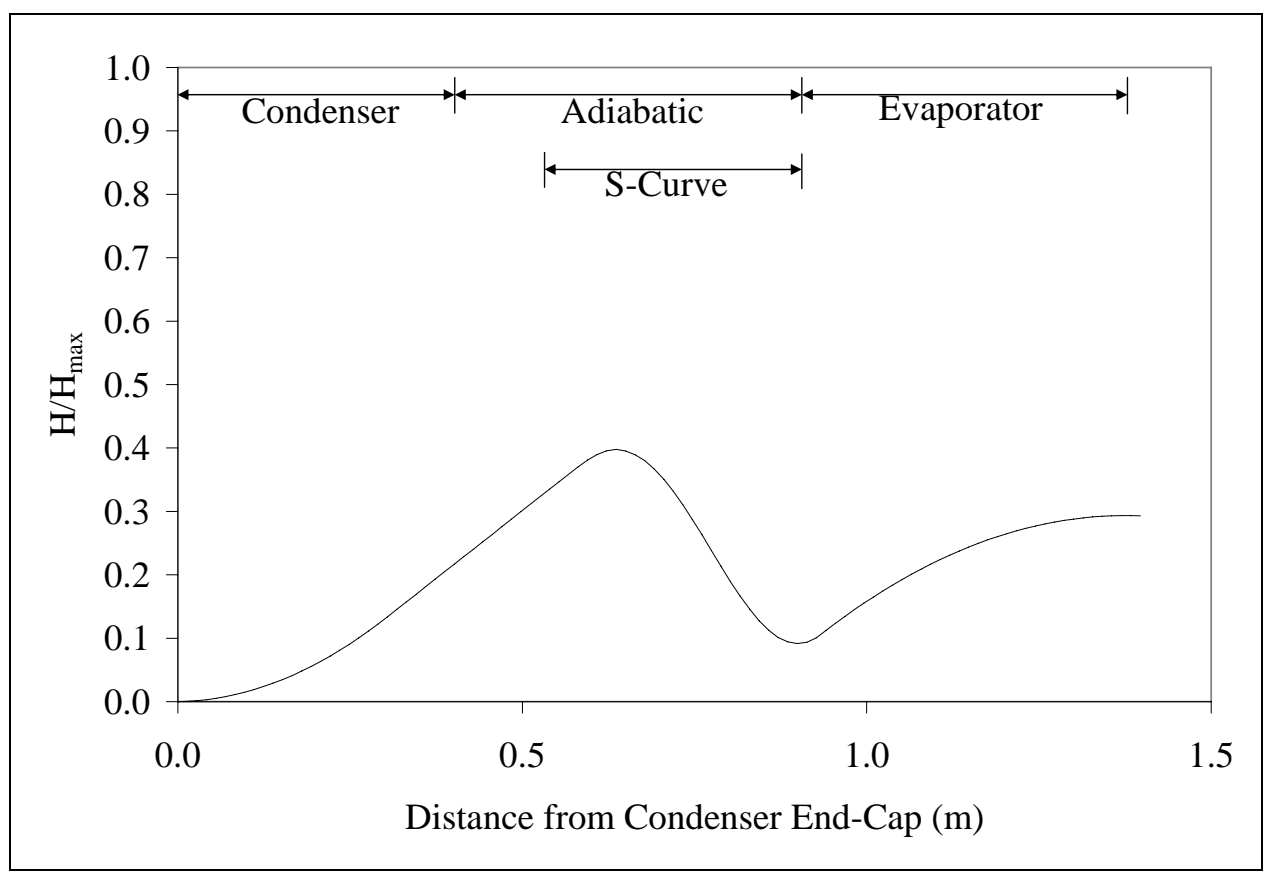

Figure 3.10. Curvature supported by the wick for the bent heat pipe at 45 rpm.

Results of a simulation with the heat pipe rotating at $200 \mathrm{rpm}$ for the $100 \mathrm{~W}$ heat load are shown in Figs. 3.11 through 3.13. Pressure distributions in the liquid and vapor are shown in Fig. 3.11. The results shown indicate the presence of the second wet-point in the adiabatic section, and a variable thickness film flow in the evaporator section with the 


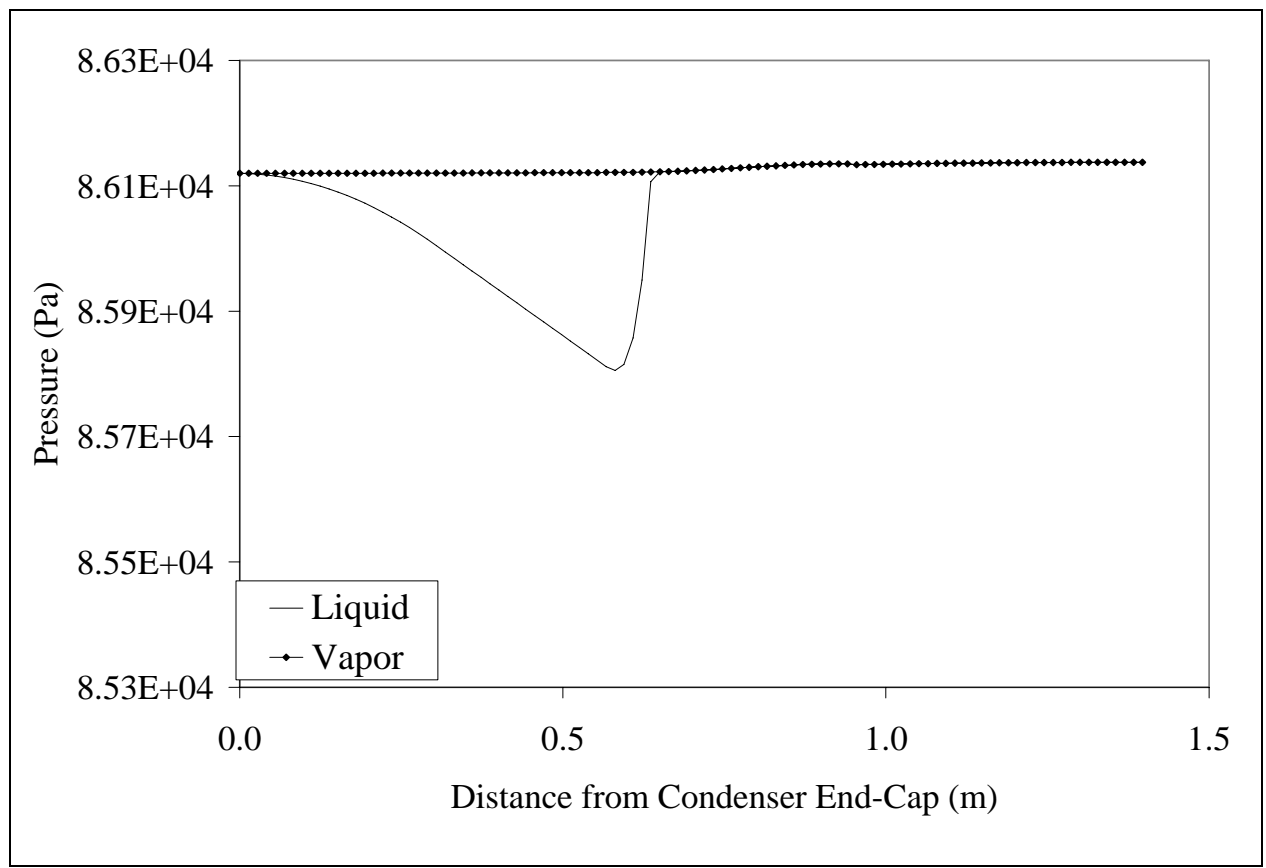

Figure 3.11. Pressure distributions for the bent heat pipe at $200 \mathrm{rpm}$.

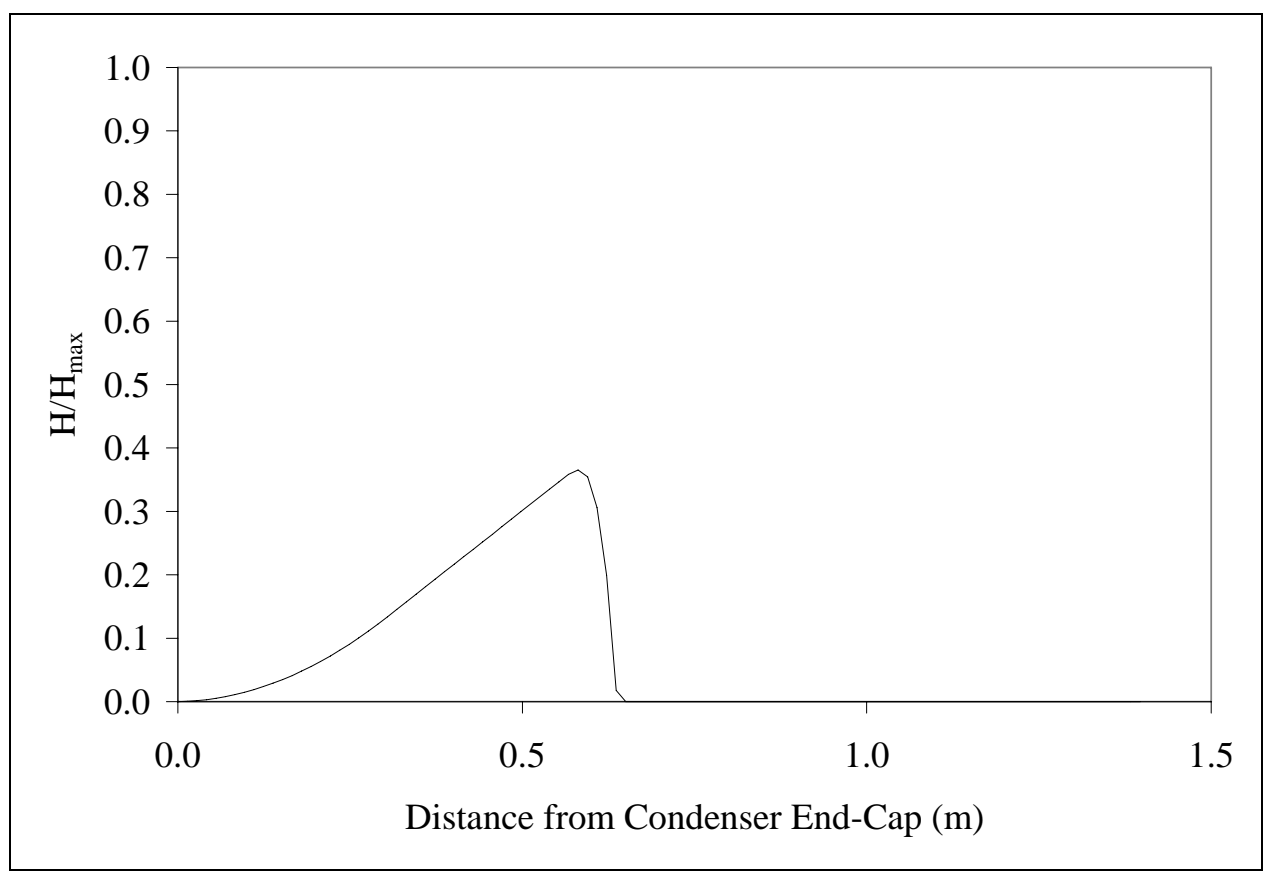

Figure 3.12. Curvature supported by the wick for the bent heat pipe at $200 \mathrm{rpm}$.

pressure of the liquid nearly equal to the pressure of the vapor (as required by the normal stress condition at the interface). In Fig. 3.13, the liquid layer thickness in the heat pipe is shown. At the second wet-point, the liquid forms the variable thickness film in the adiabatic section. It is assumed that once the liquid recedes from the wick, the excess 


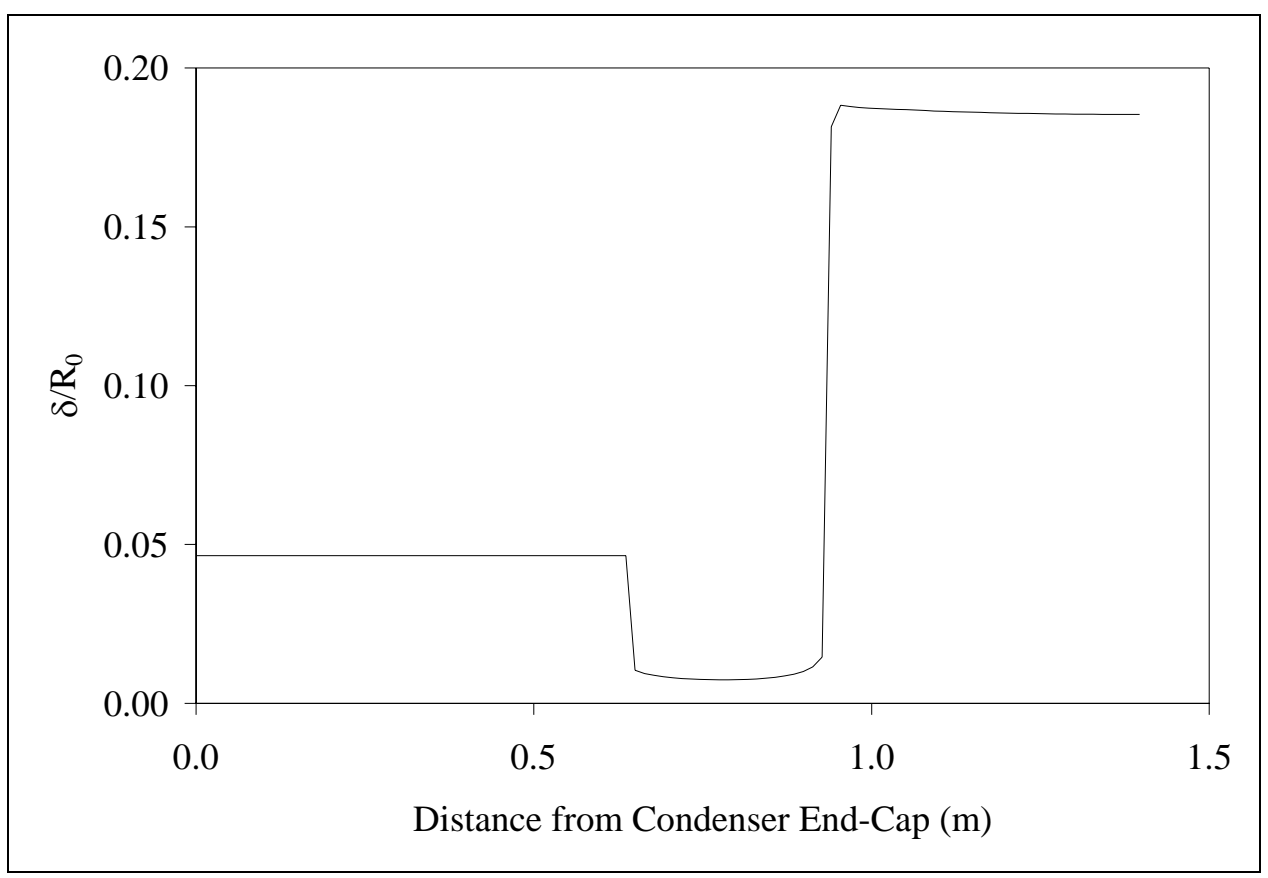

Figure 3.13. Liquid layer thickness for the bent heat pipe at $200 \mathrm{rpm}$.

fluid charge is forced into the off-axis evaporator section by the centrifugal force. The layer thickness at the entrance to the evaporator section is varied in the shooting method to ensure that the liquid volume is equal to the fluid charge. For the results presented here, the fluid charge is set equal to $15.7 \mathrm{~g}$ of methanol.

\subsection{PARAMETRIC STUDIES}

The performance of the heat pipe shown in Fig. 3.6 will be affected by a number of variables. The code developed here is used to investigate the effects that varying the heat load, rotation speed, fluid charge, and heat pipe geometry have on the temperature differences required to transfer heat through the heat pipe. In the parametric studies that follow, the baseline heat pipe has the same dimensions and fluid charge used in Sec. 3.5.

\subsubsection{Condenser Heat Transfer}

As was mentioned in the previous section, when the heat pipe shown in Fig. 3.6 is rotating, the excess liquid charge collects in the evaporator section and forms a variable- 
thickness film along the wall. Only enough liquid remains in the condenser section to fill the annular gap wick, and the wick is used to drive liquid out of the condenser.

Because the wall of the condenser is cooled, the liquid in the annulus is thermally stratified. For low flow velocities (and small film Reynolds and Péclet numbers), heat transfer across the liquid film in the condenser is primarily by pure conduction through the liquid. Consequently, the temperature difference required for heat transfer across the annulus in the condenser section should not be affected by rotation speed. Additionally, the temperature difference should linearly increase with increases in heat transfer rate. These observations are confirmed in Fig. 3.14. The baseline heat pipe is modeled for rotation speeds of 500, 700, and $1000 \mathrm{rpm}$, and with heat loads of 50, 100, and $200 \mathrm{~W}$. The figure shows that the temperature difference across the liquid film is independent of rotation speed and that doubling the heat transfer rate doubles the temperature difference.

Next, the heat pipe is modeled with a heat transfer rate of $200 \mathrm{~W}$ and a rotation speed of 1200 rpm. For the results shown in Fig. 3.15, the annular gap distance is varied from

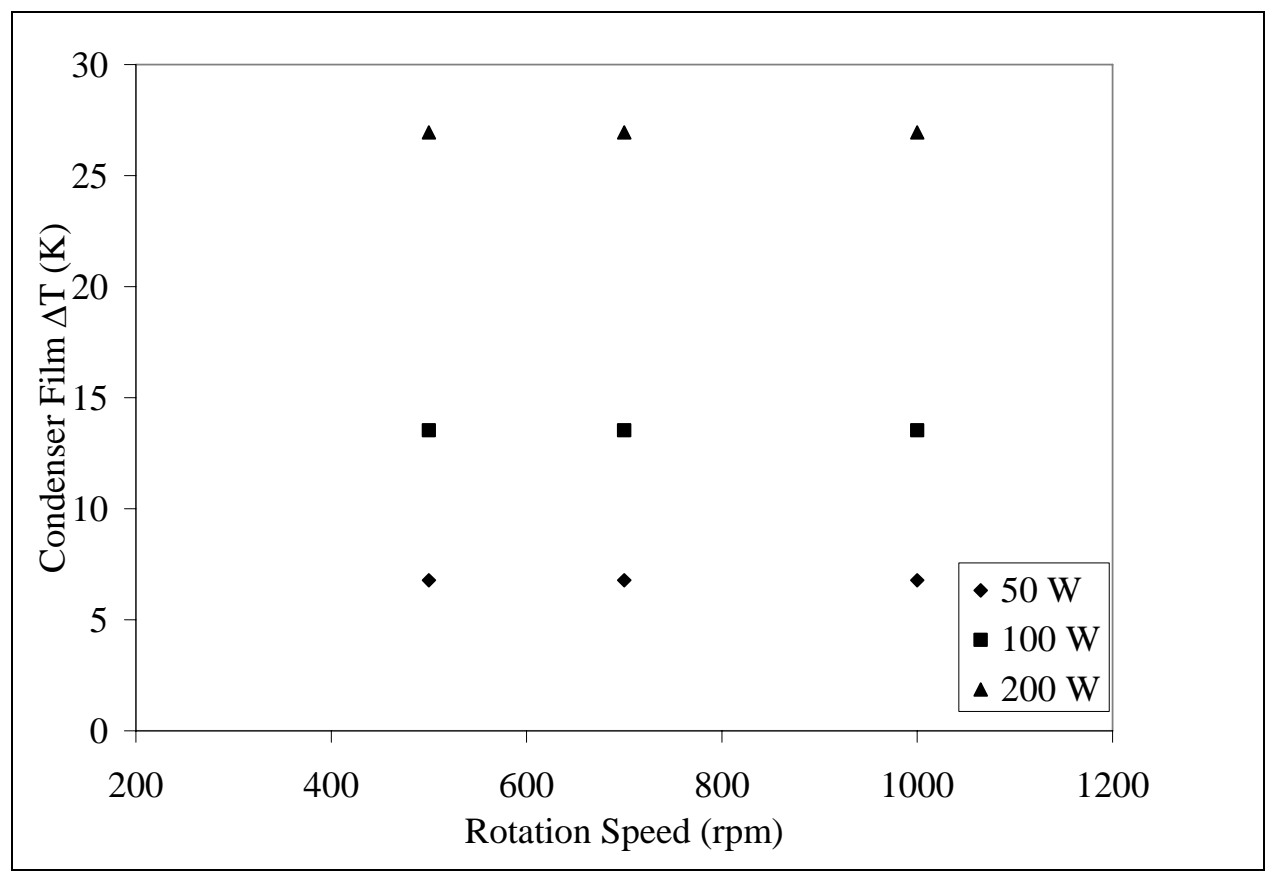

Figure 3.14. Condenser film temperature difference for the baseline heat pipe. 


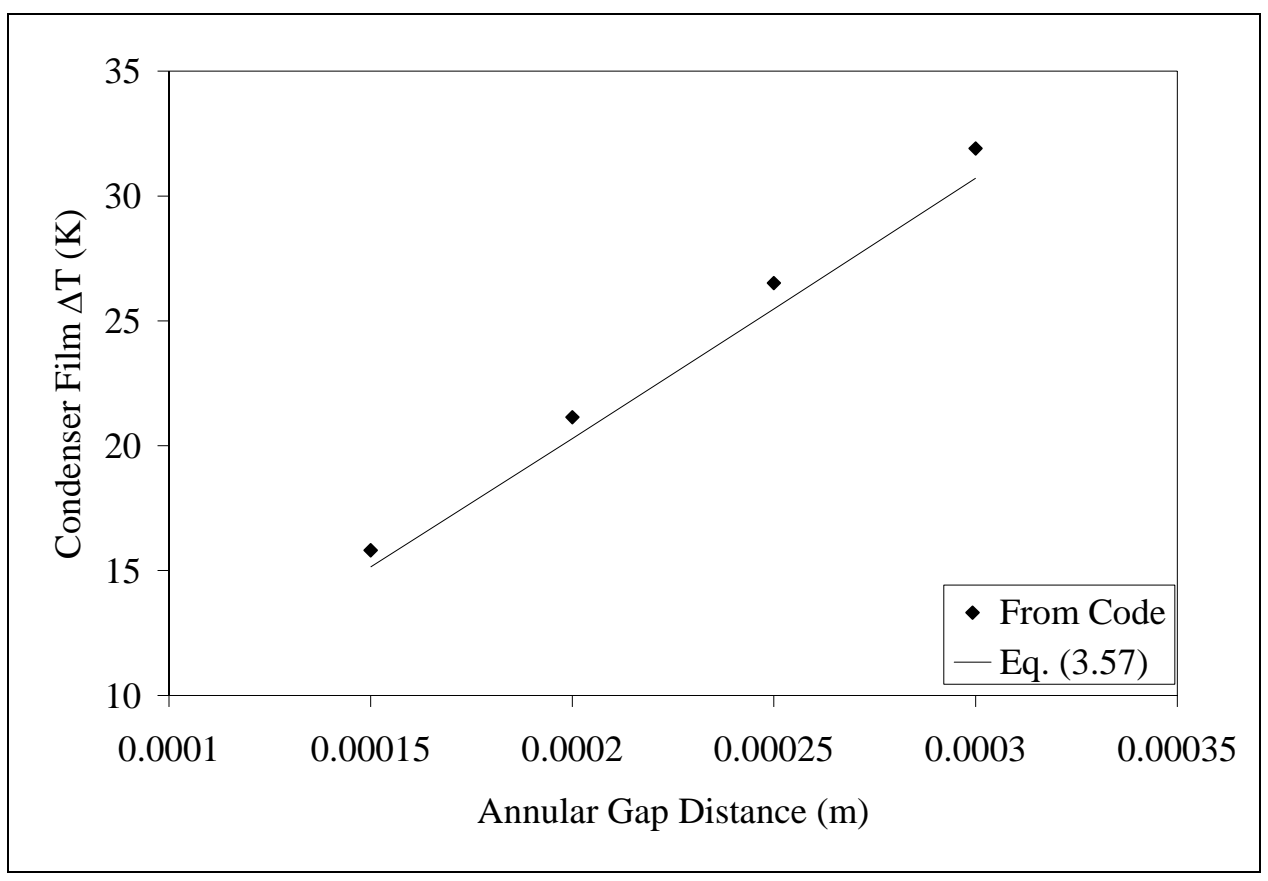

Figure 3.15. Temperature difference across the condenser film for various annular gap distances.

$0.03 \mathrm{~cm}$ to $0.015 \mathrm{~cm}$. Assuming that the heat transfer across the liquid film in the condenser is by pure conduction, the one-dimensional energy equation shows that

$$
\Delta T=-\frac{Q}{2 \pi k L_{c}} \ln (1-\delta / R) .
$$

Results obtained from the finite-difference code for the temperature difference across the liquid film in the condenser are within $5 \%$ of the results calculated with Eq. (3.62). The results indicate that heat transfer across the condenser film is primarily by conduction.

In Fig. 3.16, film Reynolds and Péclet numbers, based on the film thickness and average axial velocity in the liquid film, are shown as a function of axial length for the baseline heat pipe rotating at $1200 \mathrm{rpm}$ with a $200 \mathrm{~W}$ heat load. The film Reynolds numbers shown are much less than the value of $\operatorname{Re}_{\delta}=240$ suggested by Carpenter and Colburn as the point where the flow in an annulus will transition to turbulence [as cited in (Daniels and Al-Jumaily 1975)]. The assumption of laminar flow in the liquid is 
justified. Finally, as was shown in Figs. 3.14 and 3.15, with the modest Péclet numbers generated in the liquid, the effects of forced convection on the heat transfer across the liquid film are negligible.

Having shown that the liquid in the condenser fills the annulus and that heat transfer across the liquid annulus is governed by conduction through the liquid, the remainder of the parametric study will focus on heat transfer and fluid flow in the adiabatic and evaporator sections for the rotating heat pipe. With the heat pipe rotating, the liquid forms a variable thickness film in the adiabatic and evaporator sections, and heat transfer in the evaporator section is augmented by natural convection across the liquid film.

\subsubsection{Fixed Geometry and Fluid Charge}

The effects of heat load and rotation speed on the average temperature difference across the liquid film in the evaporator section are shown in Fig. 3.17 for the baseline heat pipe. The figure shows that, as with the axially rotating heat pipe, the temperature

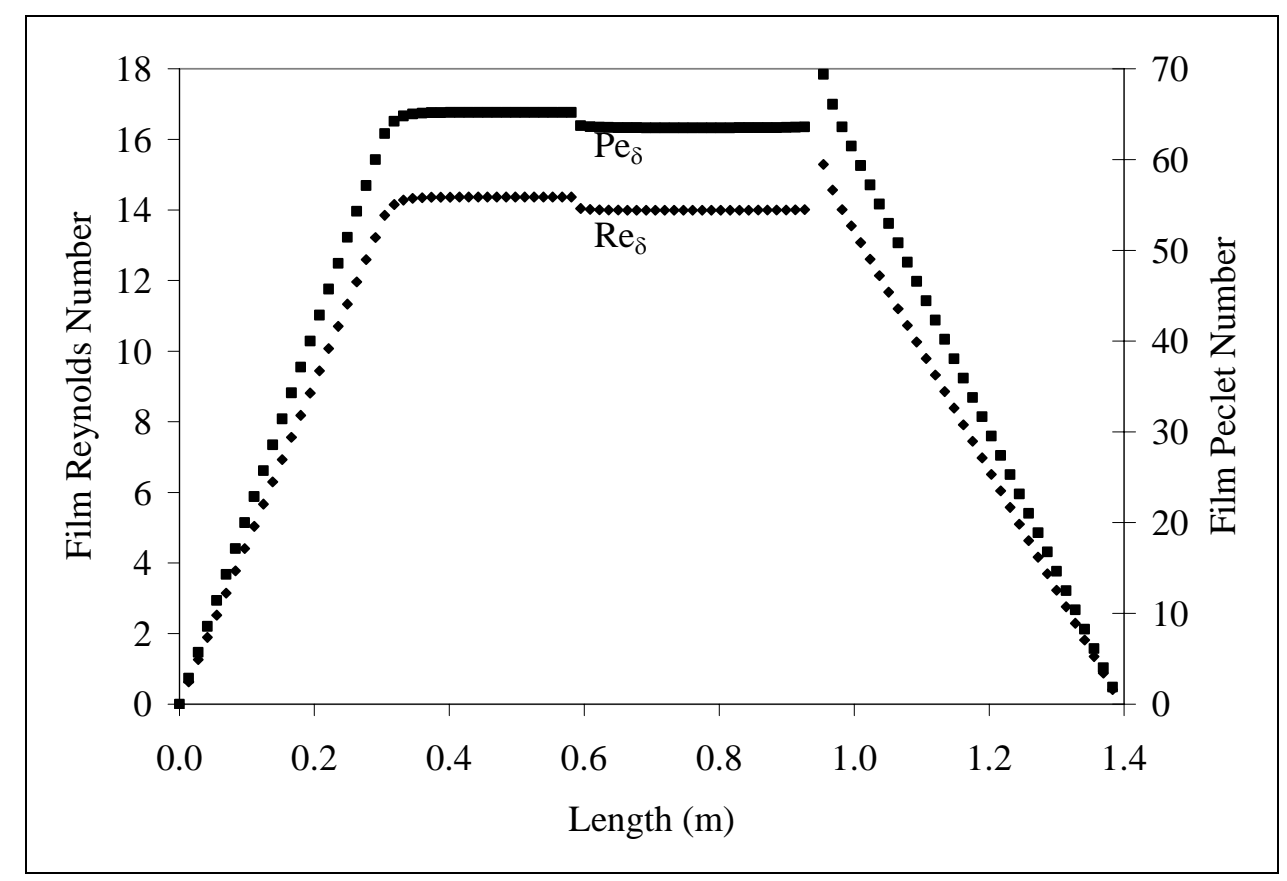

Figure 3.16. Reynolds and Péclet numbers in the liquid film for the baseline rotating heat pipe. 
difference decreases with increasing rotation speed and decreasing heat transfer rate (see Fig. 3.3). Increasing the rotation speed at a fixed heat transfer rate results in an increased Rayleigh number for the buoyancy driven convection in the evaporating liquid film. Increasing the Rayleigh number increases the heat transfer coefficient, resulting in lower temperature differences at higher rotation speeds.

\subsubsection{The Effect of Fluid Charge}

In Fig. 3.18 the fill mass (or fluid charge) is varied from 7 to $16 \mathrm{~g}$ of methanol for the baseline heat pipe rotating at $1200 \mathrm{rpm}$ with a heat load of $200 \mathrm{~W}$. Increasing the fluid charge in the heat pipe will increase the thickness of the liquid film in the evaporator section.

With a small fluid charge, $<8.6 \mathrm{~g}$, the Rayleigh number in the evaporator film is below the critical value of $R a_{c}=1600$ for an evaporating liquid film predicted by Jankowski et al. (Jankowski et al. 2006a). Below this critical value, heat transfer across

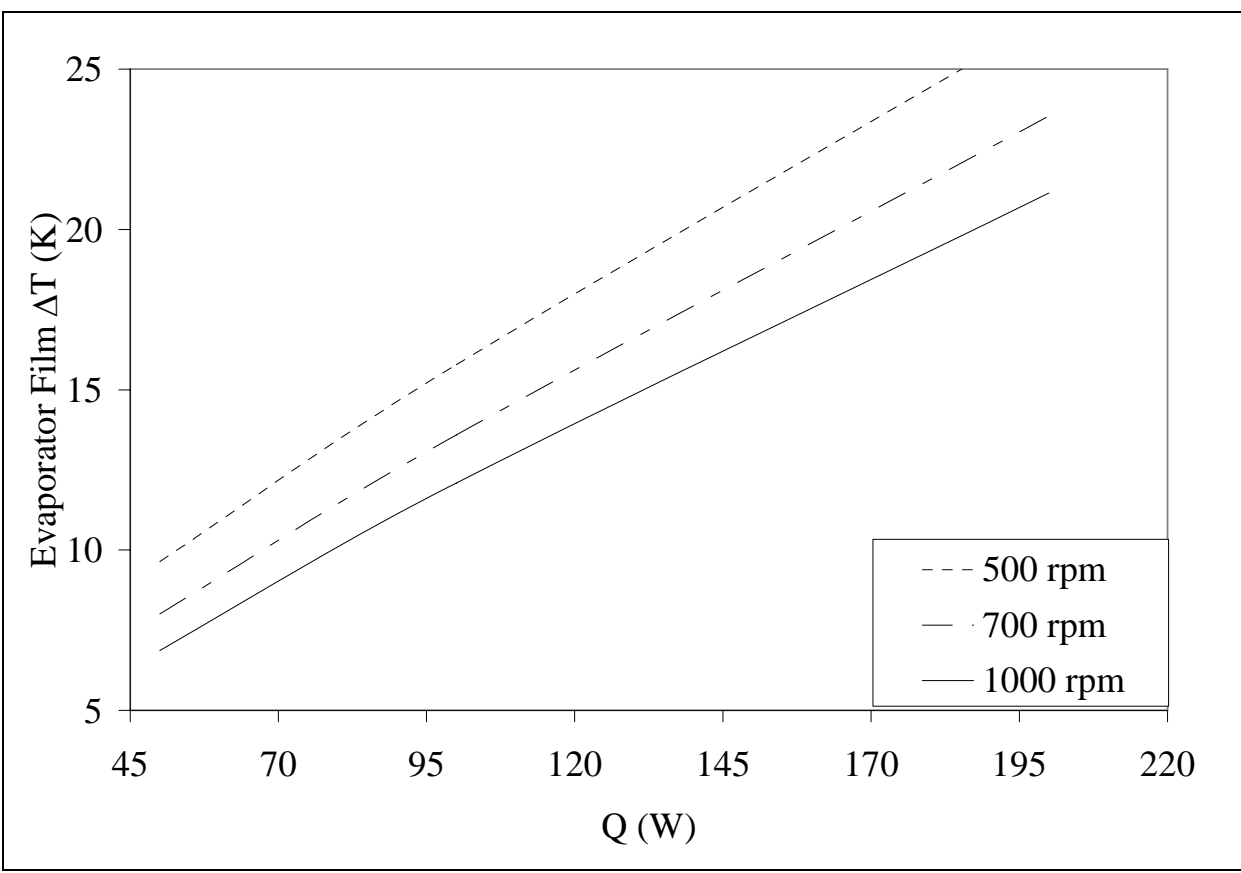

Figure 3.17. Temperature difference across the evaporator film for various heat loads and rotation speeds. 


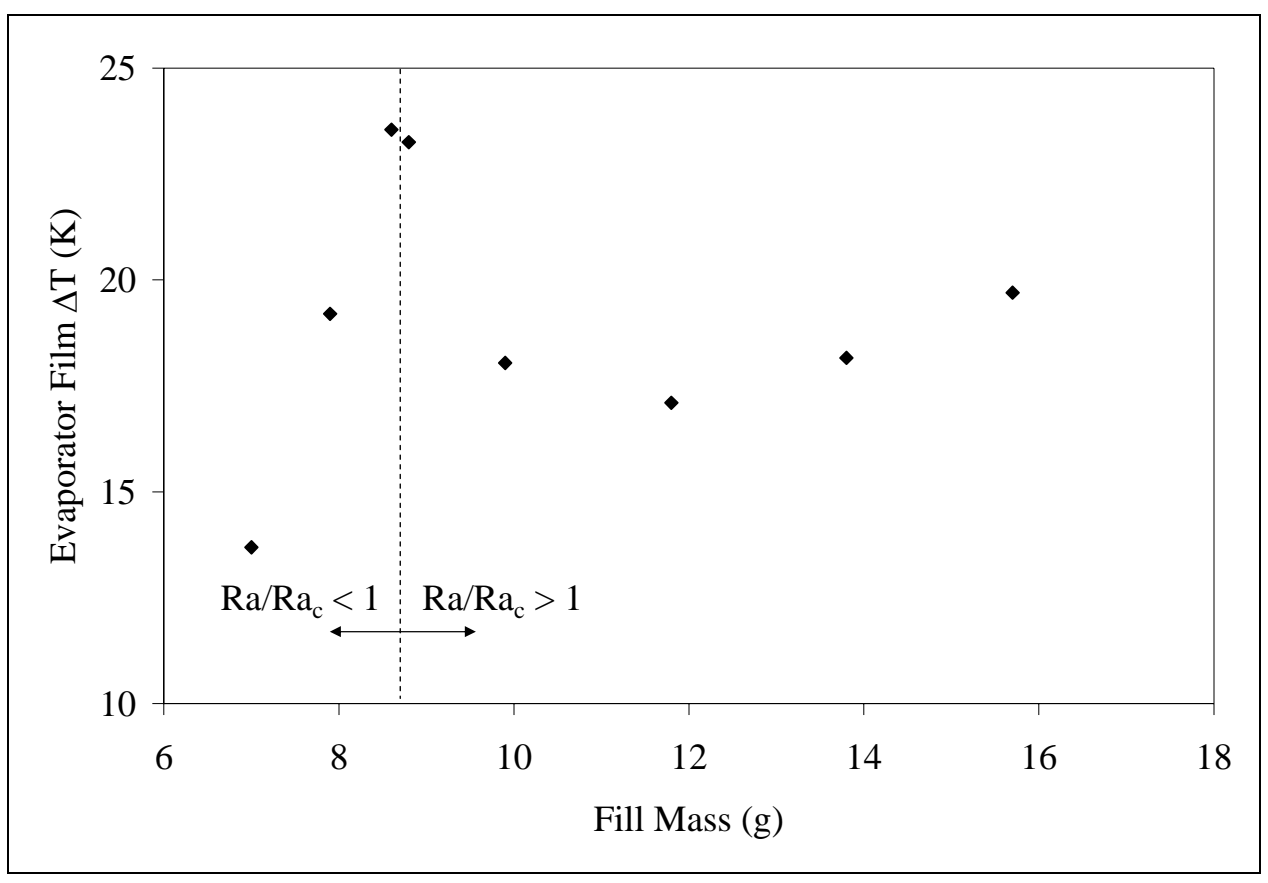

Figure 3.18. Evaporator film temperature difference as the fill mass is varied.

the liquid film is by conduction, and increases in the fluid charge result in increased temperature differences.

As the fluid charge is increased, and the evaporator film thickness increases, natural convection is present in the liquid film. After reaching the critical Rayleigh number, the temperature difference decreases with increasing fluid charge, reaches a minimum, then increases.

A scaling argument can be used to understand this behavior. For a fixed heat transfer rate, the temperature difference is related to the Nusselt number and liquid layer thickness by $\Delta T \sim \delta / N u$. The Nusselt number may be expressed as $N u \sim R a^{n}$, so that $N u \sim \delta^{3 n}$ and $\Delta T \sim \delta^{1-3 n}$. This relationship shows that the exponent $n$ at a given point on the $\mathrm{Nu}$ vs. $R a$ curve will determine whether the temperature difference increases or decreases with layer thickness. For $n>1 / 3$, the temperature difference will decrease with increasing layer thickness, while for $n<1 / 3$ the opposite will occur. Close to the critical 
Rayleigh number, where the slope of the $N u$ vs. $R a$ curve is highest (see Fig. B.6), the exponent $n$ is large, resulting in decreasing temperature difference with increasing fluid charge just after the onset of convection. Further increases in fluid charge and Rayleigh number decrease the exponent $n$ below $1 / 3$.

\subsubsection{INCREASING THE LENGTH OF THE EVAPORATOR}

In Fig. 3.19, the length of the evaporator is increased from the baseline value of 45.7 $\mathrm{cm}$ to $183 \mathrm{~cm}$ for the heat pipe rotating at $1200 \mathrm{rpm}$ with a $200 \mathrm{~W}$ heat load. When increasing the length of the evaporator, the fluid charge is also increased so that the liquid layer thickness in the evaporator remains constant. In the absence of natural convection with a constant layer thickness, the temperature difference across the liquid film would decrease linearly as the length of the evaporator is increased. The non-linear behavior observed in Fig. 3.19 can, once again, be attributed to natural convection in the liquid

film. For a constant layer thickness and heat transfer rate, $\Delta T \sim 1 /\left(L_{e} N u\right)$ and

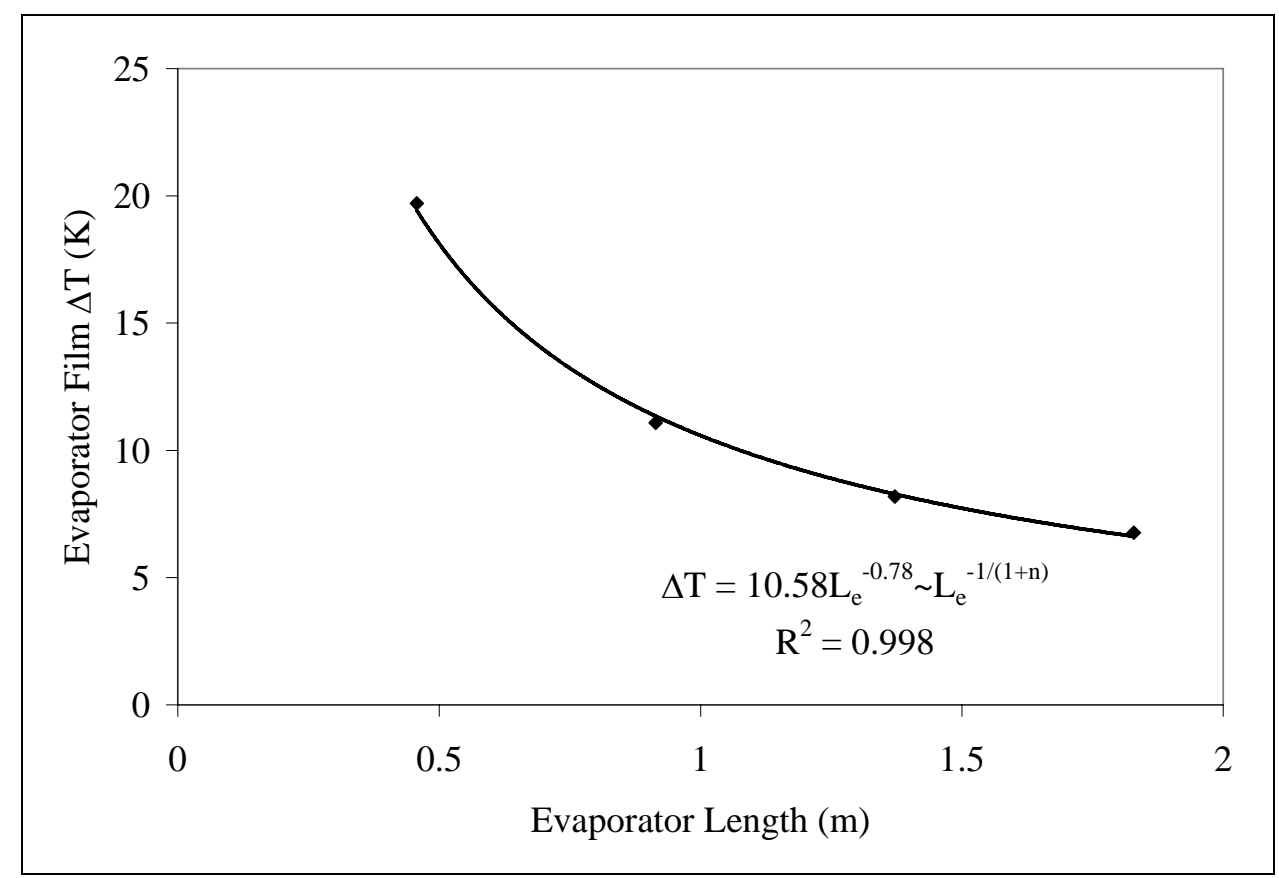

Figure 3.19. The effect of evaporator length on temperature difference across the evaporator film for a constant liquid layer thickness. 
$N u \sim \Delta T^{n}$, so that $\Delta T \sim L_{e}^{-1 /(1+n)}$. From the curve fit in Fig. 3.19, $n=0.28$, which is a reasonable value for laminar natural convection (Bejan 1995).

\subsubsection{InCREASING THE Distance FROM THE Axis of Rotation}

The distance between the axis of rotation and the off-axis evaporator section, $d$, is varied from $12.1 \mathrm{~cm}$ to $96.5 \mathrm{~cm}$ in Fig. 3.20 for the heat pipe rotating at $1200 \mathrm{rpm}$ with a $200 \mathrm{~W}$ heat load. Increasing the distance from the axis of rotation increases the centrifugal force acting on the vapor. As suggested by models of radially rotating heat pipes, the pressure of the vapor at the off-axis entrance to the adiabatic section must be large enough to overcome the centrifugal force as the vapor flows to the on-axis condenser (Ling and Cao 2000; Ling et al. 2001). The hydrostatic pressure rise across the S-shaped adiabatic section is given by

$$
\Delta p_{v}=\frac{1}{2} \rho_{v} \omega^{2} d^{2}
$$

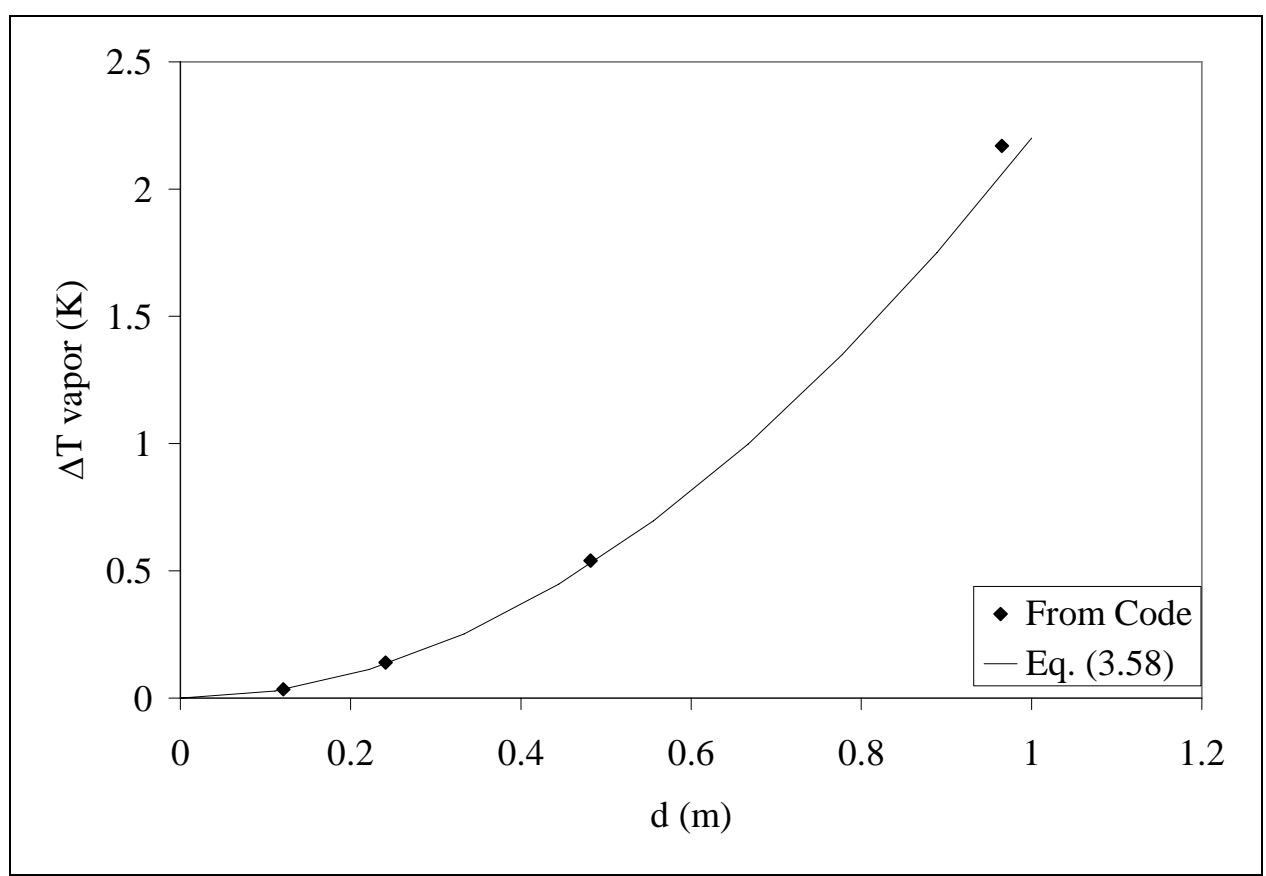

Figure 3.20. Temperature difference over the adiabatic section as the distance from the off-axis evaporator to the on-axis condenser is increased. 
For saturated vapor, the pressure difference predicted by Eq. (3.63) can be used to calculate a temperature difference across the adiabatic section. For the curve shown in Fig. 3.20, saturated methanol properties have been determined using Engineering Equation Solver (EES) software (F-Chart). Fig. 3.20 shows that the finite-difference code is properly calculating the pressure rise in the vapor due to the centrifugal force. The finite-difference analysis is predicting a slightly higher (1\%) pressure rise due to friction acting on the vapor, which is not accounted for in Eq. (3.63).

\subsection{CONCLUDING REMARKS}

The verification and validation studies indicate that the heat pipe code developed in this chapter is able to reproduce results from existing models of both rotating and stationary heat pipes. Additionally, the parametric studies have shown that the model is predicting the expected behavior for the proposed heat pipe. The analysis code will be used in Chapter 5 to develop performance maps for the heat pipe with the S-shaped curve and results of the analysis will be compared to experimental data. 


\section{Heat Pipe Test apparatus}

The numerical model in the previous chapter assumed that the flow of liquid was symmetric about the longitudinal axis of the heat pipe. Although the axisymmetric model does incorporate many aspects of the flow, three-dimensional behavior is expected in the actual heat pipe. At high rotation rates, the liquid in the off-axis revolving evaporator section will pool onto one side of the heat pipe. An experimental apparatus for testing the proposed rotating heat pipe has been designed and built (Jankowski et al. 2006b). The goal of the experimental study is to ensure that the two-dimensional model is able to capture the key parameters affecting the performance of the rotating heat pipe.

The various components of the experimental apparatus will be described in this chapter. A method for fabrication of the heat pipe with the annular gap wick structure and a procedure for cleaning and filling the heat pipe with the working fluid will be outlined. Finally, the rotating test rig will then be described, along with safety analyses that were used in sizing many of the components of the experimental setup.

\subsection{FABRiCATION of THE HEAT Pipe}

The heat pipes used throughout the experiments were fabricated from a 0.5” (1.27 cm) OD, 0.035” $(0.089 \mathrm{~cm})$ wall thickness copper tube. The wick was made from two layers of 200x200 mesh stainless steel screen. To fabricate the wick, the screen is cut into $1 ”(2.54 \mathrm{~cm})$ wide strips and the strips are wound around a $10 \mathrm{~mm}$ brass mandrel on a 30 degree bias. The bias angle is chosen so that each wrap of screen slightly overlaps the previous wrap. At the overlap region, the stainless steel screen is spot-welded to itself. A photograph of a partially completed wick, showing the bias wrapped screen, is in Fig. 4.1. After completing the first layer of bias wrapped screen, a second layer is 
wrapped over the first. The use of the $10 \mathrm{~mm}$ brass mandrel and a two-layer bias wrapped screen gives a nominal annulus thickness of 0.01 inches $(0.25 \mathrm{~mm})$.

Once the wick was fabricated, it was removed from the brass mandrel, and a test to determine the effective pore radius (or maximum curvature) was performed. To determine the pore radius, both ends of the wick are plugged, and the wick is immersed in a methanol bath. Immersing the wick in the methanol bath causes methanol to be held in pores of the wick by surface tension, and air to be trapped in the interior vapor space of the wick. The plug at one end of the wick has a fill tube protruding from it, which allows gas to be transferred from outside the methanol bath to the interior vapor space of the wick. During the pore test, the fill tube is connected to a pressure gauge and a nitrogen gas bottle. A metering valve is used to slowly increase the pressure in the vapor space of the wick by bleeding in nitrogen gas. The pressure is increased until vapor bubbles escaping through the surface of the wick are observed. The pressure at which vapor bubbles break through the wick can be used, along with the normal stress condition (which, in the hydrostatic case, reduces to the Laplace-Young equation), to determine an

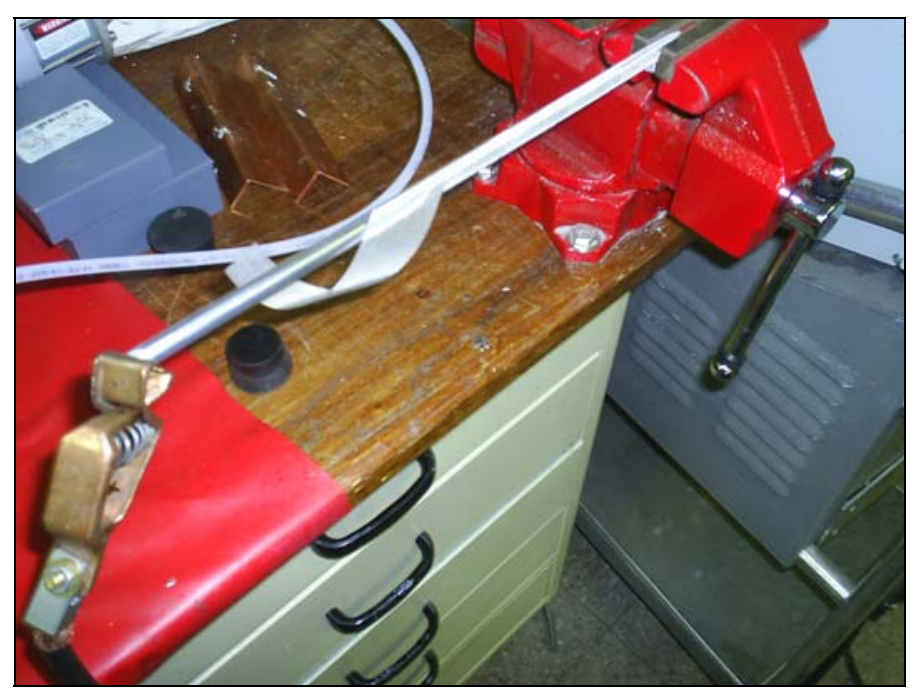

Figure 4.1. The bias wrapped wick. 
effective pore radius of the porous wick (since the surface tension of methanol is known). For the $200 \times 200$ mesh screen wick, the pore radius was measured as $r_{\text {eff }}=44 \mu \mathrm{m}$. This value compares well with the $r_{\text {eff }}=42 \mu \mathrm{m}$ expected for the screen (Unique).

After the wick has been pressure tested the wick is inserted into the copper tube. A $10 \mathrm{~mm}$ diameter $1 \mathrm{~cm}$ long brass plug is then soldered into the evaporator end of the wick. This plug in the evaporator end seals the vapor space in the evaporator, allowing the capillary pressure difference to be supported at the evaporator end during stationary operation. The plug has a 1/8” hole drilled through its center. A 1/8” copper fill tube is soldered into the plug. Copper plumbing caps are then soldered onto the evaporator and condenser ends of the heat pipe. The cap at the end of the evaporator also has a 1/8” hole drilled into it so that the fill tube can protrude out. The heat pipe fabrication is completed by attaching a fill valve to the end of the fill tube.

With the ends of the heat pipe sealed by the soldered copper caps, the heat pipe is evacuated and helium leak checked. A funnel is attached to the fill valve, and the liquid methanol working fluid is added to the funnel. The heat pipe is charged by opening the fill valve and letting a fixed volume of methanol into the evacuated heat pipe. This methanol charge is emptied from the heat pipe, and the heat pipe is purged with nitrogen gas and re-evacuated several times. This filling, emptying, and pumping and purging cycle is repeated many times to clean the interior of the heat pipe before the final fluid charge is added.

With the heat pipe fabricated and filled, the two bends were put into the heat pipe to produce the shape shown in Fig. 3.6. Two tube benders have been fabricated for the purpose of making the S-shaped curve in the heat pipe. 


\subsection{Rotating Test Apparatus}

The general arrangement of the rotating test apparatus is shown in Fig. 4.2. The motor drive system, condenser bath, heat pipes, and the supports for the rotating heat pipes are mounted on a frame made from 2" x 2" hollow steel channel with a 0.125 ” wall thickness. The rotating center shaft, on which two rotating heat pipes and the condenser bath are mounted, is a solid three inch diameter hardened steel shaft.

The center shaft is supported by three bearings. At the two ends of the shaft, which have been turned down to a diameter of 1", self-adjusting double-row ball bearings are used. The bearing near the center of the shaft is a 5" diameter single row ball bearing. An aluminum sleeve, with an outside diameter of 5", fits over the 3" diameter center shaft. Four set screws are used to hold the position of the aluminum sleeve relative to the rotating center shaft, and the outer diameter of the sleeve is pressed into the inner race of the large bearing. Two 5/8” diameter holes are drilled into the aluminum sleeve allowing the heat pipes to pass through the sleeve.

A support structure, made from eight separate pieces of $1 / 2$ " thick G-10 which are

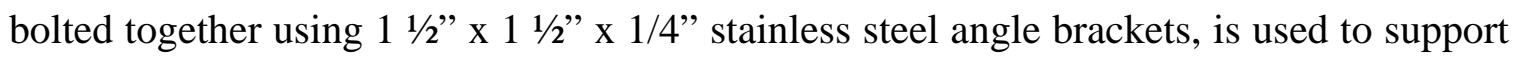
the revolving evaporator section of the heat pipes. The position of the G-10 support structure, which is shown in Fig. 4.3, is fixed relative to the shaft using eight set screws. A 1/16" thick stainless steel shield, also shown in Fig. 4.3, is then attached to the outer diameter of the G-10 support structure. The purpose of the shield is to reduce the drag force on the G-10 structure as it rotates through the air.

The condenser ends of the heat pipes are cooled in the water jacket near the drive motor. The heat pipes and center shaft pass through an aluminum sleeve similar to the 


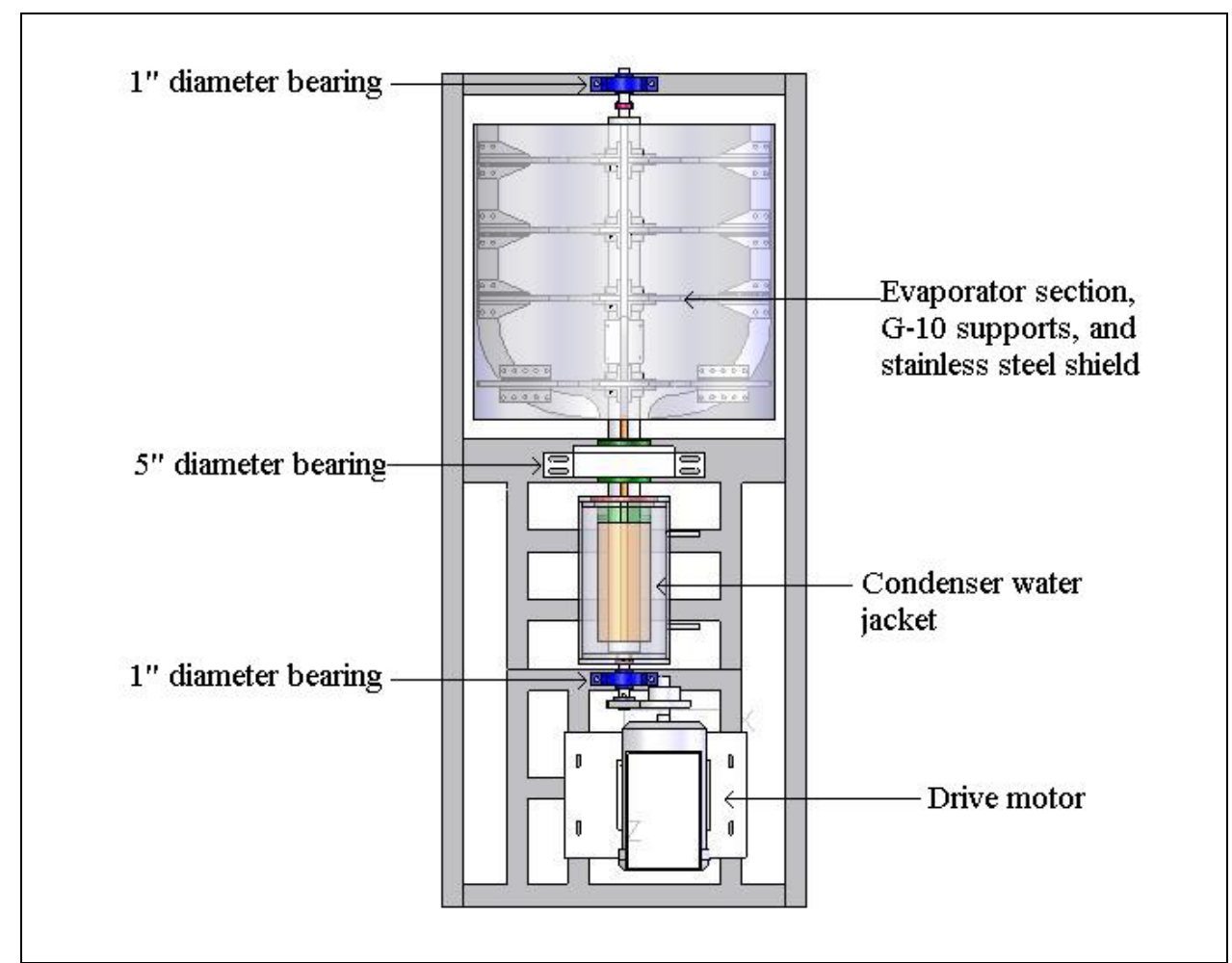

(a)

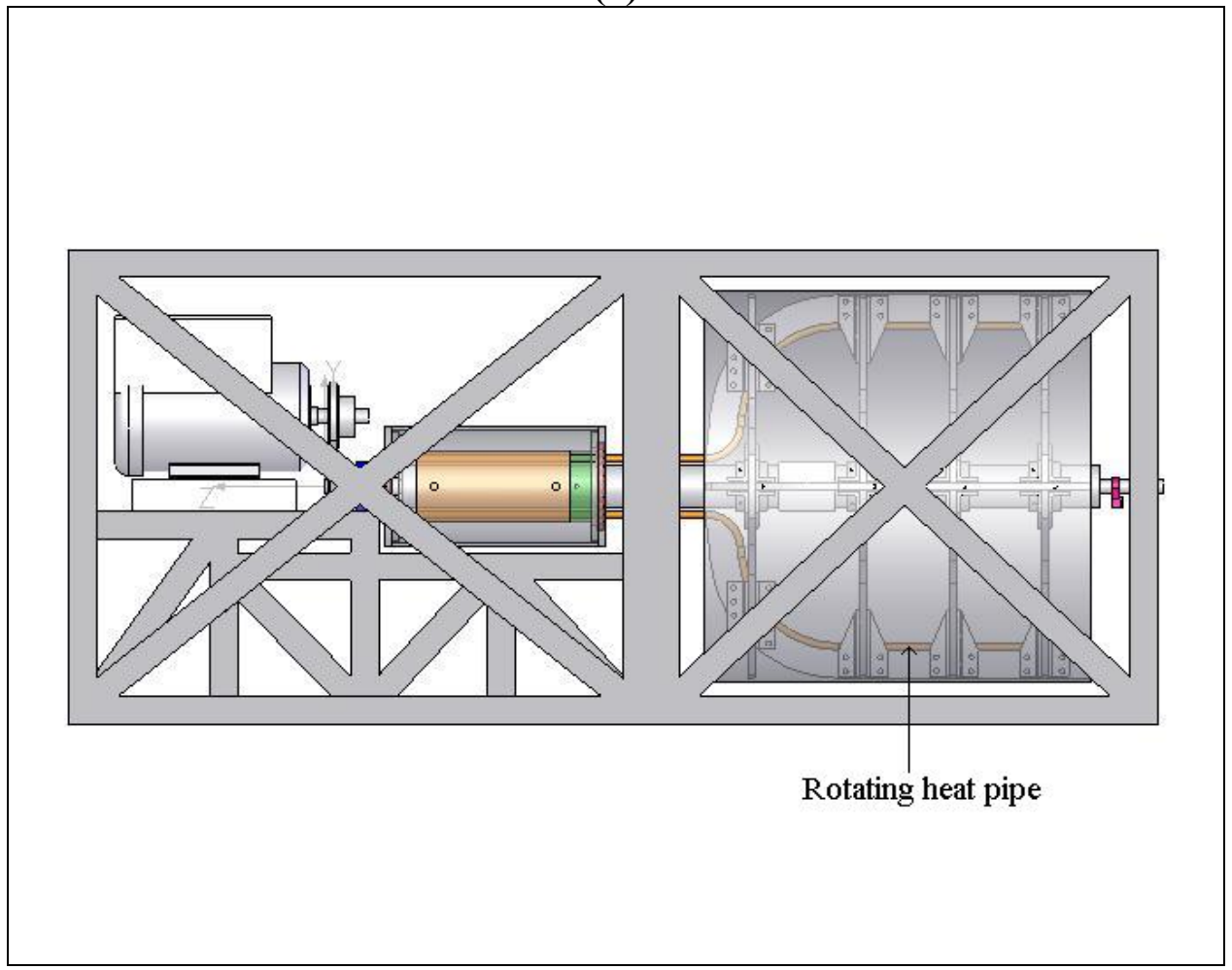

(b)

Figure 4.2. The rotating test apparatus. (a) Top and (b) side views are given. 


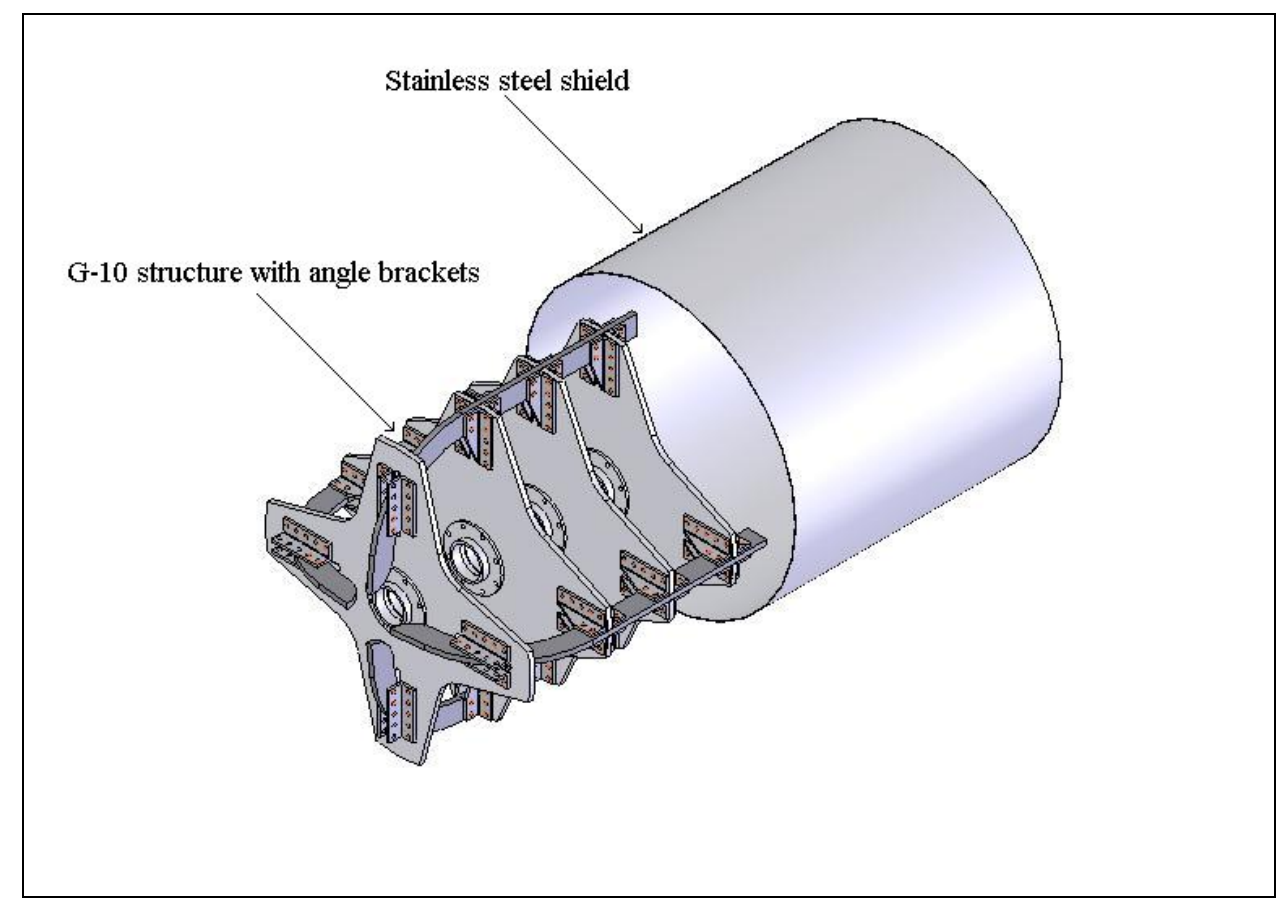

Figure 4.3. G-10 support structure and stainless steel shield.

sleeve used in the large bearing. Between the outer diameter of the rotating sleeve and the stationary water jacket, a 5" diameter friction seal is used. The heat pipes are held in place relative to the rotating shaft using solid copper sleeves of similar design to those used for the large bearing.

The motor used to drive the rotating apparatus is a $5 \mathrm{hp}$ variable speed alternating current motor (Baldor). The motor has a speed range from 0 to $3450 \mathrm{rpm}$. The sheaves used on the motor and center shaft have a ratio of 1:2.25 allowing the shaft to be rotated at a maximum speed of $1500 \mathrm{rpm}$.

The water jacket used to cool the condenser section is filled with water with a small amount of rust inhibitor. A recirculator/chiller with a control temperature range from -15 to 85 degrees Celsius is used to circulate coolant through the water jacket.

The evaporator ends of the heat pipes are heated using 1 inch wide Kapton tape heaters. A total of 18 inches in length of the evaporator is heated. The maximum power 
density of the tape heaters is $10 \mathrm{~W} / \mathrm{in}^{2}$, allowing for a maximum heat load of $180 \mathrm{~W}$ to be supplied to each heat pipe. Power is supplied to the heaters through a slip ring assembly mounted to the 1 " diameter portion of the center shaft at the evaporator end of the rotating apparatus.

Temperatures along the length of the heat pipes are measured using type $\mathrm{K}$ thermocouples that are recorded using two eight channel remote data loggers made by Omega Engineering Inc. (Omega). The data loggers can be run remotely, so that thermocouple signals do not need to be passed through a slip ring assembly. The data obtained on the data loggers, however, must be downloaded after rotation of the apparatus is stopped.

Calibration tests were performed with the data loggers and thermocouples to quantify the uncertainty in the temperature measurements. The thermocouples were immersed in an ice-water bath and in boiling water. Data from the calibration tests are shown in Fig. 4.4. Data were taken every ten seconds from all eight thermocouples for twenty minutes. This gives a sample size of 960 readings. Both the ice-water and boiling water tests show normal frequency distributions, with the boiling water test having the largest standard deviation. A band of $\pm 0.5 K$ corresponds to a 99 percent ( \pm 3 standard deviations) confidence interval for the boiling water test. This estimate of the uncertainty is used throughout the experimental study.

Safety shields are attached to the support frame. Steel plates with a thickness of 5/16” are used on the two sides of the support frame, 5/8” aluminum plates are used on the top, and 1/2" thick LEXAN plates are used on the ends. The top plates slide beneath two steel rails running the length of the support frame. This allows the top plates to be 


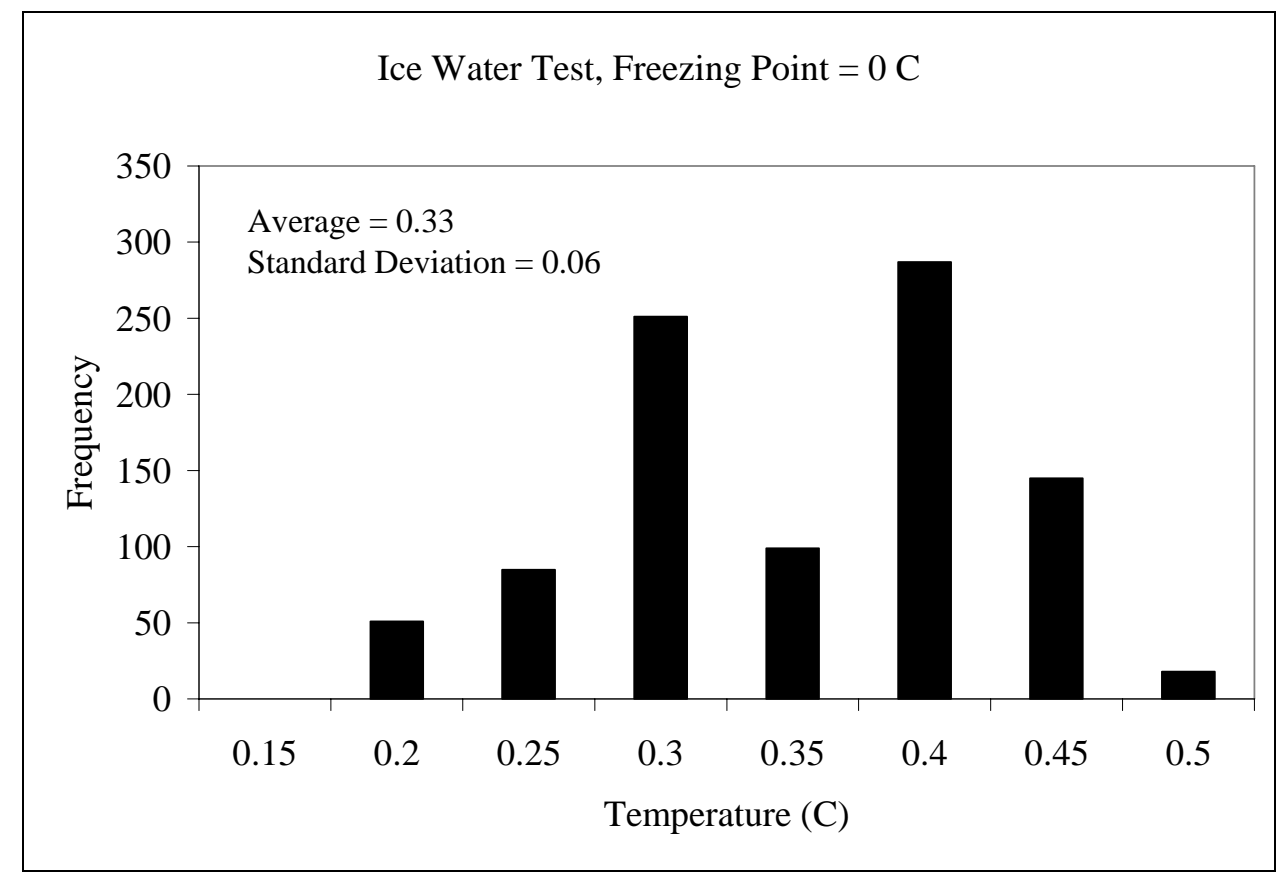

(a)

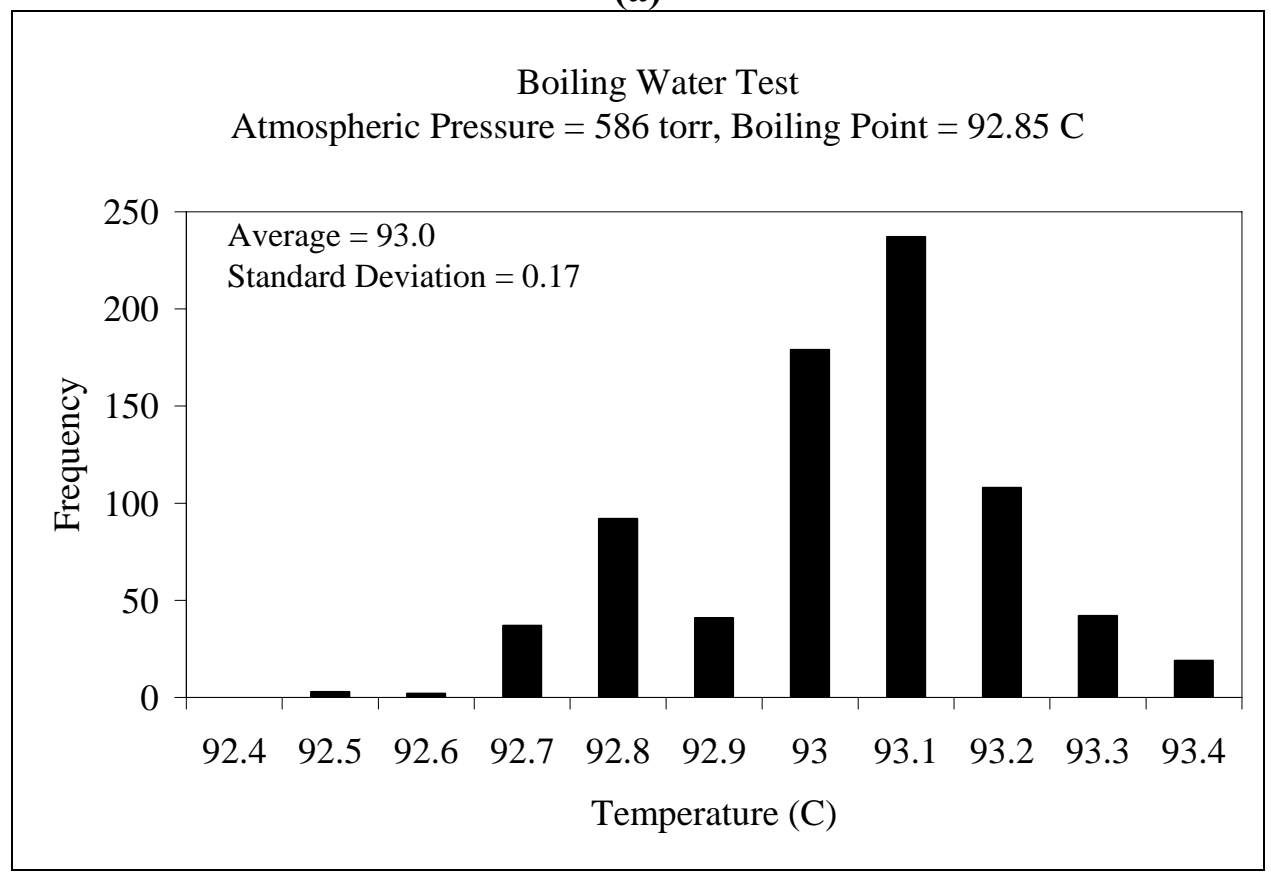

(b)

Figure 4.4. Data from calibration tests of the thermocouple measurements in (a) ice-water and (b) boiling water.

taken off of the apparatus for data collection, maintenance of the apparatus, and to make necessary adjustments. The shields are in place to prevent the possibility of projectiles from becoming a hazard to personnel in the area. 
Some pictures of the apparatus at various stages of completion are shown in Fig. 4.5.

\subsection{SUPPORTING ANALYSES}

A structural analysis of the G-10 supports and a safety analysis of the shielding were performed. The apparatus was originally designed to rotate to a maximum speed of 5600

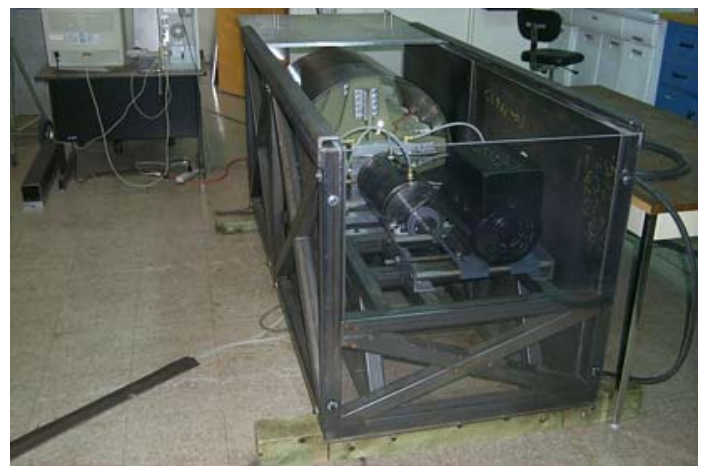

(a)

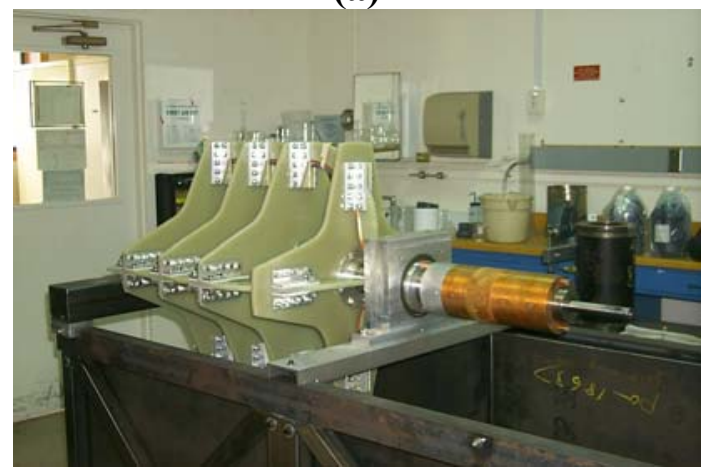

(c)

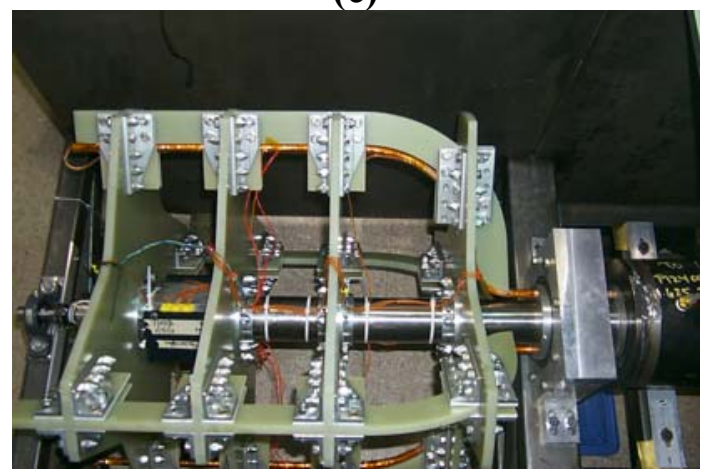

(e)

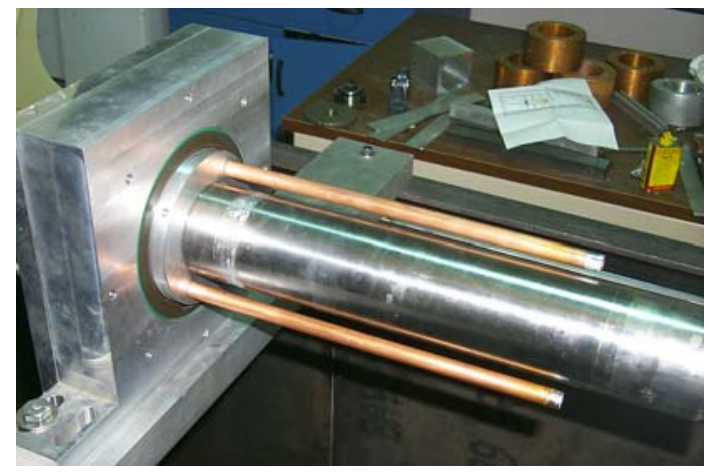

(b)

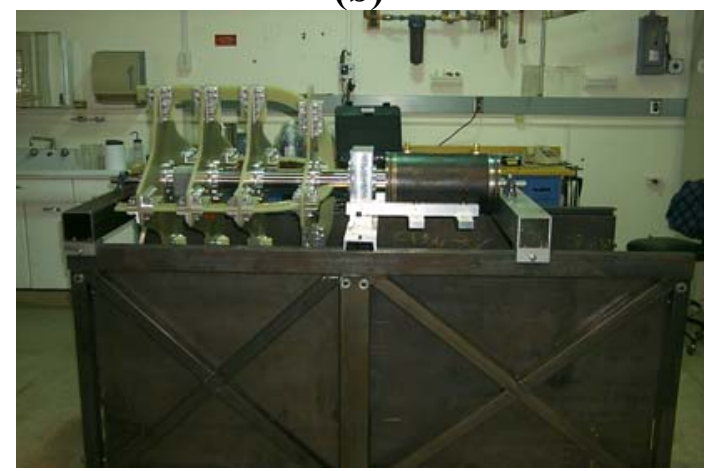

(d)

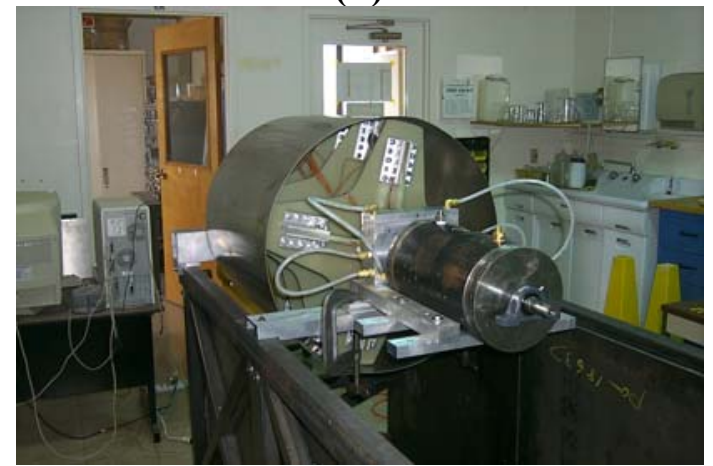

(f)

Figure 4.5. The test apparatus during assembly. In (a) a view of the completed assembly in the frame is shown. Figures (b), (c), and (d) show the condenser end assembly. A close-up of the large bearing with the condenser sections extending out is in (b), the condensers with the copper cover blocks are shown in (c), and in (d) the

water jacket has been mounted at the condenser end. Figure (e) shows the evaporator sections and G-10 supports and (f) shows the assembly with the stainless steel shield. 
rpm. After assembly, however, it was found that the motor was undersized for the rotating load. Both the structural and shielding analyses assume a maximum speed of $5600 \mathrm{rpm}$.

The thickness of the steel and LEXAN shields were determined using the Thor equations database (Greenspon 1976; Prenger 2003). The projectile analysis assumed that a 1" diameter 2" long particle that is located 9" from the axis of rotation becomes detached from the rotating apparatus while the apparatus is rotating at $5600 \mathrm{rpm}$. This would simulate one of the bolts on the G-10 structure coming loose. The shield thickness was determined so that in the event of a projectile being generated, this particle would be confined inside of the shielding.

A structural analysis of the G-10 support structure was performed using the COSMOS finite element analysis software (Hill 2003). The analysis was performed to aid in designing the geometry of the G-10 pieces and the brackets used to join the G-10 pieces together. The maximum stress generated in the G-10 supports was monitored for the structure rotating at $5600 \mathrm{rpm}$. The design was determined so that the maximum stress was below the yield strength of G-10. 


\section{Results}

Results of the experimental investigation are compared to predictions from the numerical model in this chapter. The experiments were carried out in three steps. After the heat pipe was assembled and before bending to make the S-shaped curve, performance tests aimed at determining the capillary limit of the straight heat pipe were completed. Following these performance limit tests, the two bends making up the Sshaped curve were incorporated into the heat pipe, and the performance tests were repeated. These tests allow for a quantification of the effect that the bends have on the stationary heat pipe performance and are used to show that the wick structure was not damaged during bending. Finally, the rotating test apparatus was assembled with the heat pipes and data was obtained with the heat pipes operating at rotation speeds up to 1200 rpm.

\subsection{STATIONARY BENCH-TOP TESTS}

During fabrication of the heat pipe, bench-top performance tests were performed to determine the heat transfer limitation of the heat pipe. For this long $(140 \mathrm{~cm})$, small diameter $(1.3 \mathrm{~cm})$ heat pipe with the annular gap wick, the capacity of the heat pipe is limited by the capillary limit. A picture of the bench-top test stand with the straight heat pipe is shown in Fig. 5.1. To test the capillary limit, the heat pipe was artificially loaded against gravity with the evaporator at a higher elevation than the condenser. This artificial loading decreased the amount of power that had to be put through the heat pipe to reach the capillary limit. In the bench-top tests, the condenser end of the heat pipe was cooled by a copper chill-block clamped to the condenser end of the heat pipe, with water circulating through the block from a recirculating water bath. With the condenser 


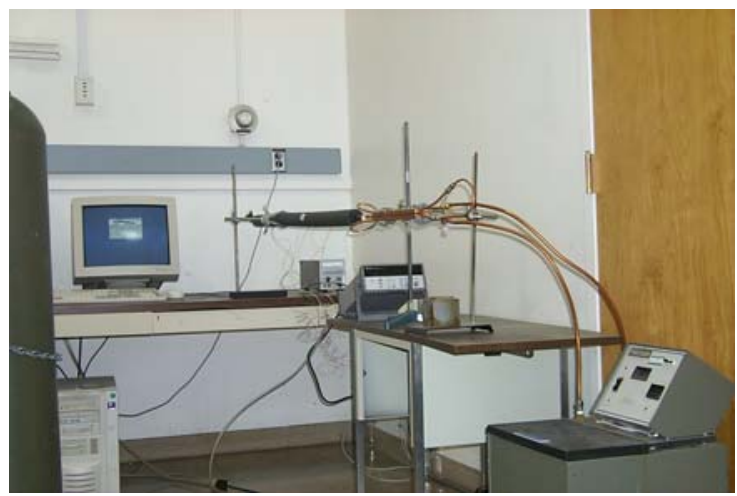

(a)

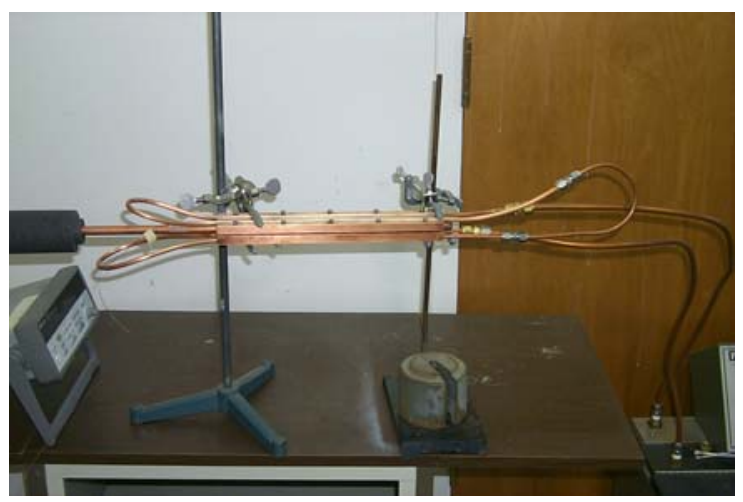

(b)

Figure 5.1. The bench-top test stand with the straight heat pipe. The overall set-up is shown in (a), and the copper chill-block on the condenser is shown in (b)

temperature set by the water bath, the power input to the tape heater on the evaporator end of the heat pipe was incrementally increased until a dry-out was observed. Dry-out of the evaporator, caused by the capillary limit, was observed in the data as a sharp increase in the evaporator temperature soon after the power to the heat pipe was increased. As shown in the example data file of Fig. 5.2, dry-out occurs when the temperature at the end of the evaporator (TC 1) increases rapidly after the power input is increased to $16.2 \mathrm{~W}$, indicating that the wick dries out between 14.5 and $16.2 \mathrm{~W}$.

Dry-out data for the heat pipe, before and after bending are shown in Fig. 5.3 with a 21 g pentane charge. The heat loads presented in the figure have been corrected for heat loss through the $1.27 \mathrm{~cm}$ thick foam insulation on the evaporator and adiabatic sections. At the highest temperature data point $(50 \mathrm{C}$ ), heat loss through the insulation is $7 \mathrm{~W}$. The data indicate satisfactory agreement between the tests and model predictions, with a reduction in performance caused by placing the bends in the heat pipe. It was found that adding an effective length of 20 outer tube radii to the length of the adiabatic section allowed the model results to simulate the performance of the bent heat pipe. This effective length is of the same order of magnitude as empirical correlations for the effect 


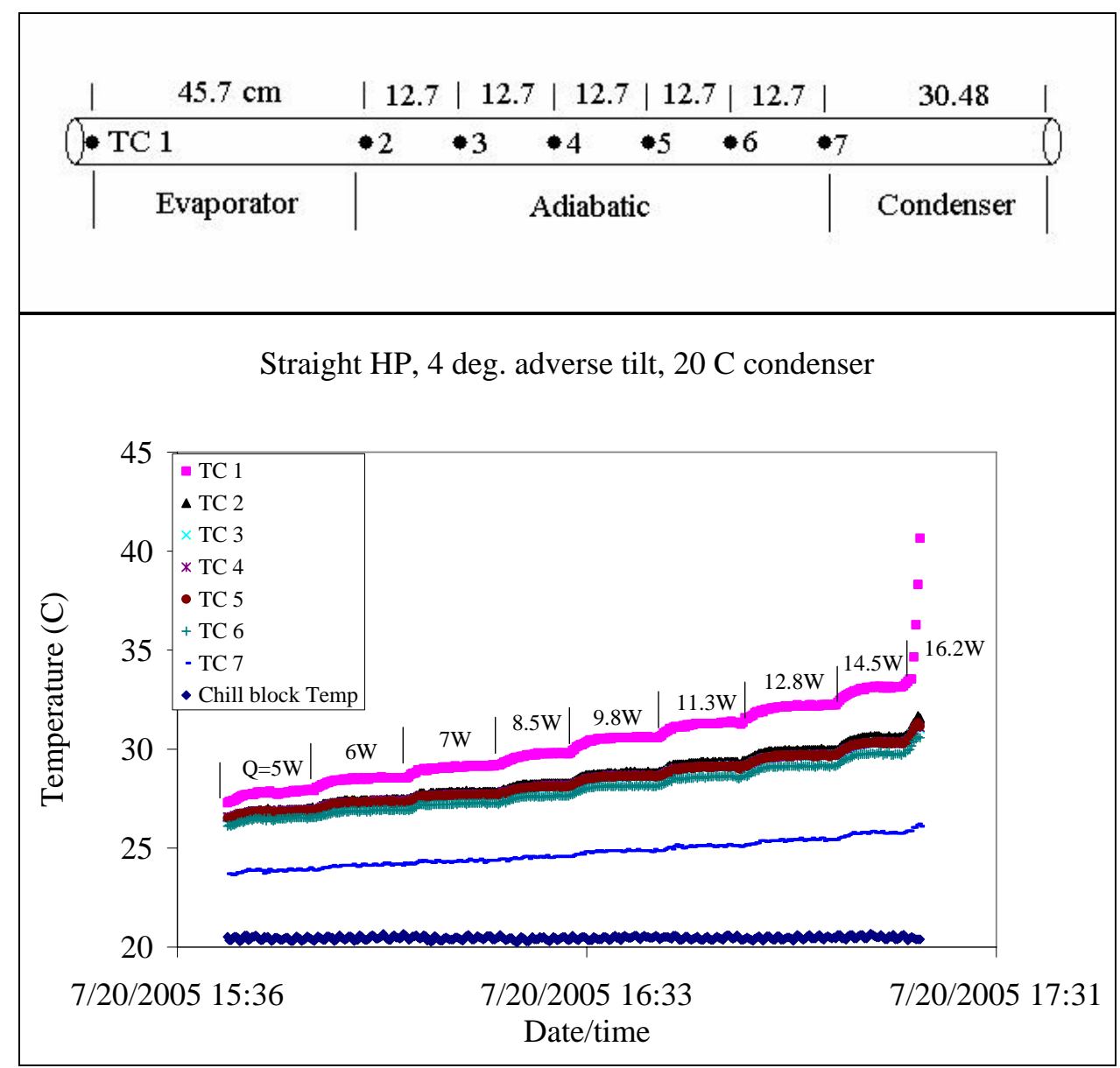

Figure 5.2. Example data file for the stationary test with the straight heat pipe with the condenser set to $20 \mathrm{C}$.

of large radius 90 degree bends in tubing (Eaton 2003). The stationary bench-top tests indicate that the wick was not damaged during the fabrication and bending processes.

\subsection{Tests in the Rotating Apparatus}

\subsubsection{Reported UnCERTAinties}

Two major sources of uncertainty in reporting the test results for the rotating heat pipe in the test apparatus have been identified. Temperature values along the length of the heat pipe and temperature differences between two locations on the heat pipe are reported. As was mentioned in the previous chapter, all individual thermocouple readings are assumed to have an uncertainty of $\pm 0.5 \mathrm{~K}$. Additionally, heat transfer rates 


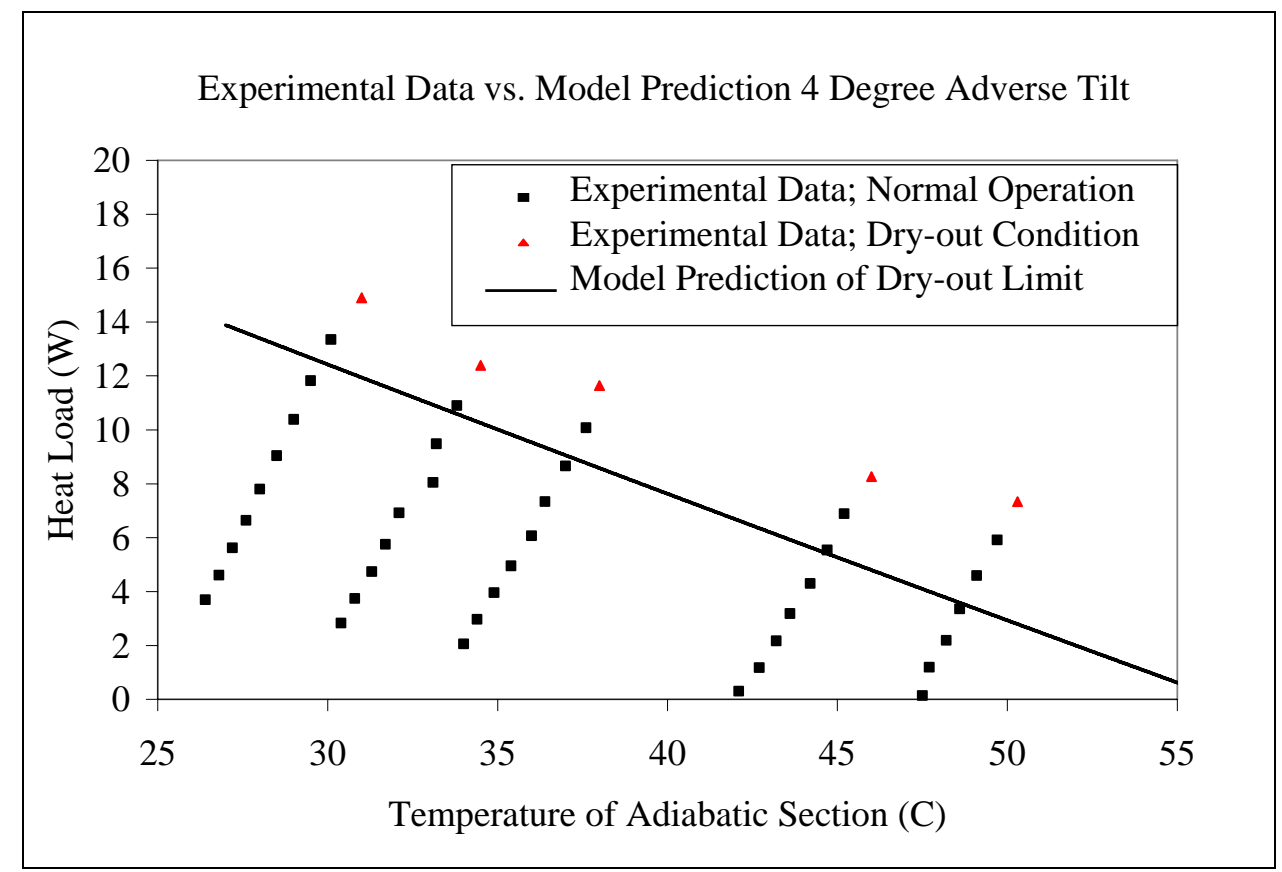

(a)

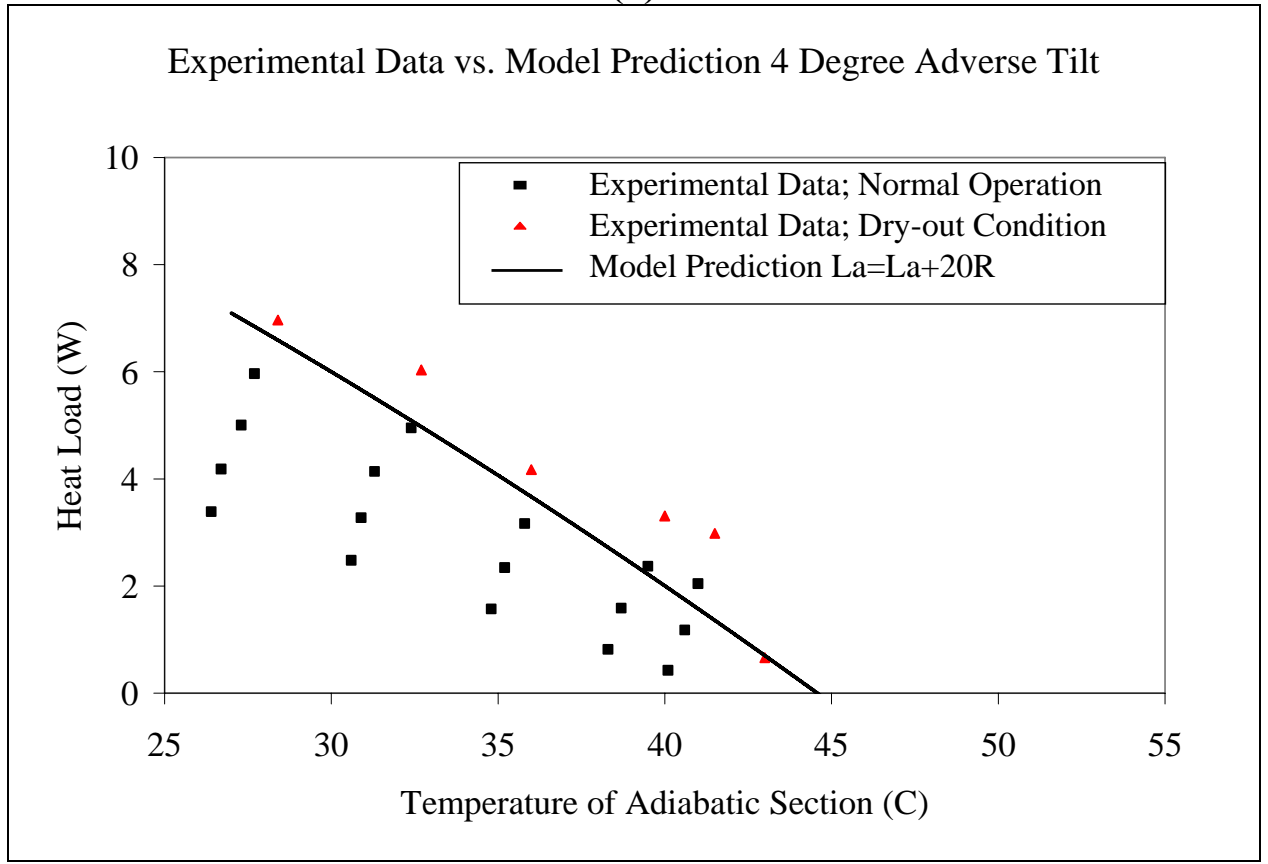

(b)

Figure 5.3. Bench-top test results (a) before bending and (b) after bending.

are reported. The maximum value of the heat transfer rate into the evaporator section is given by the power dissipated in the resistance heater. The heater resistance and voltage drop across the heater are measured with a multi-meter, allowing for a calculation of the maximum heat transfer rate to within $1 \%$. The major source of uncertainty in the heat 
transfer rate into the heat pipe is due to heat loss through the insulation and G-10 supports. A conservative (high) estimate of the conduction loss has been made by assuming that the outer surfaces of the insulation and G-10 in contact with the evaporator and adiabatic sections are at room temperature, and that the surface temperature of the heat pipe is equal to the temperature of the evaporator. Figure 5.4 shows the calculated heat loss as a function of heat pipe surface temperature. These calculated values are used as an estimate of the uncertainty in reporting the heat transfer rate.

Uncertainties for results calculated from the measured values are determined with standard formulas for uncertainty propagation (Figliola and Beasley 1995). Sample calculations are given in Appendix C.

\subsubsection{Stationary TESTS}

After completing the bench-top tests, the heat pipes were assembled into the rotating test apparatus. To assemble the apparatus, the heat pipes had to be partially disassembled (the condenser end-cap had to be taken off). Stationary (non-rotating) tests were repeated with the heat pipes in the rotating test apparatus to ensure that the heat pipe would perform as expected. Results of the stationary tests in the rotating apparatus are shown in Fig. 5.5. The dry-out data, obtained for the heat pipe filled with $15.7 \mathrm{~g}$ of methanol, are compared to model results. Again, the data indicate that the wick structure was not damaged during assembly of the rotating test apparatus.

\subsubsection{Low SpeEd Dry-Out Tests}

Low speed tests $(\omega \leq 100 \mathrm{rpm})$ were performed to determine how the capillary limit is affected by the centrifugal force. For the heat pipe with the off-axis evaporator section, 


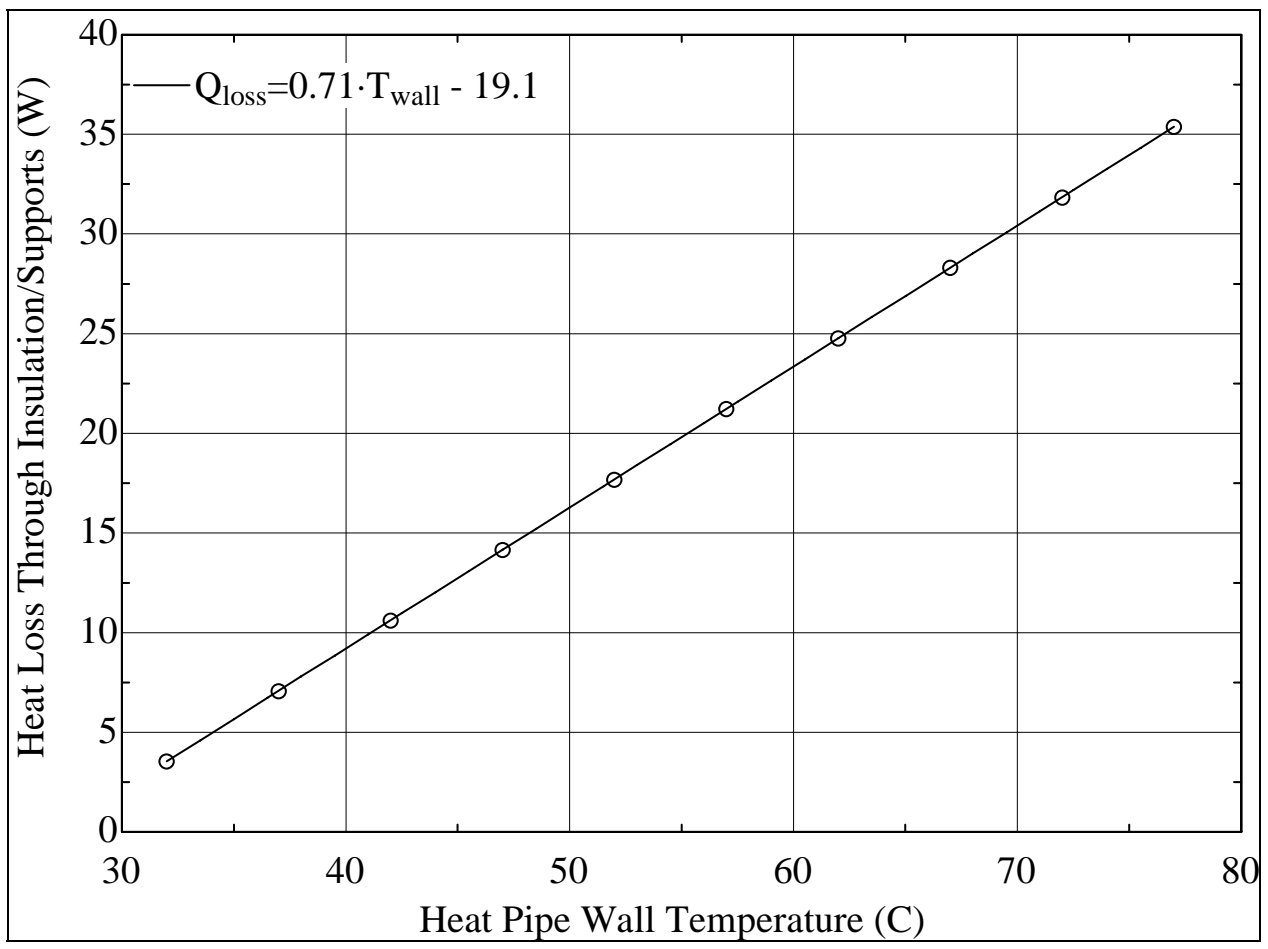

Figure 5.4. Estimated heat loss through the G-10 supports and insulation for the heat pipe installed in the rotating apparatus.

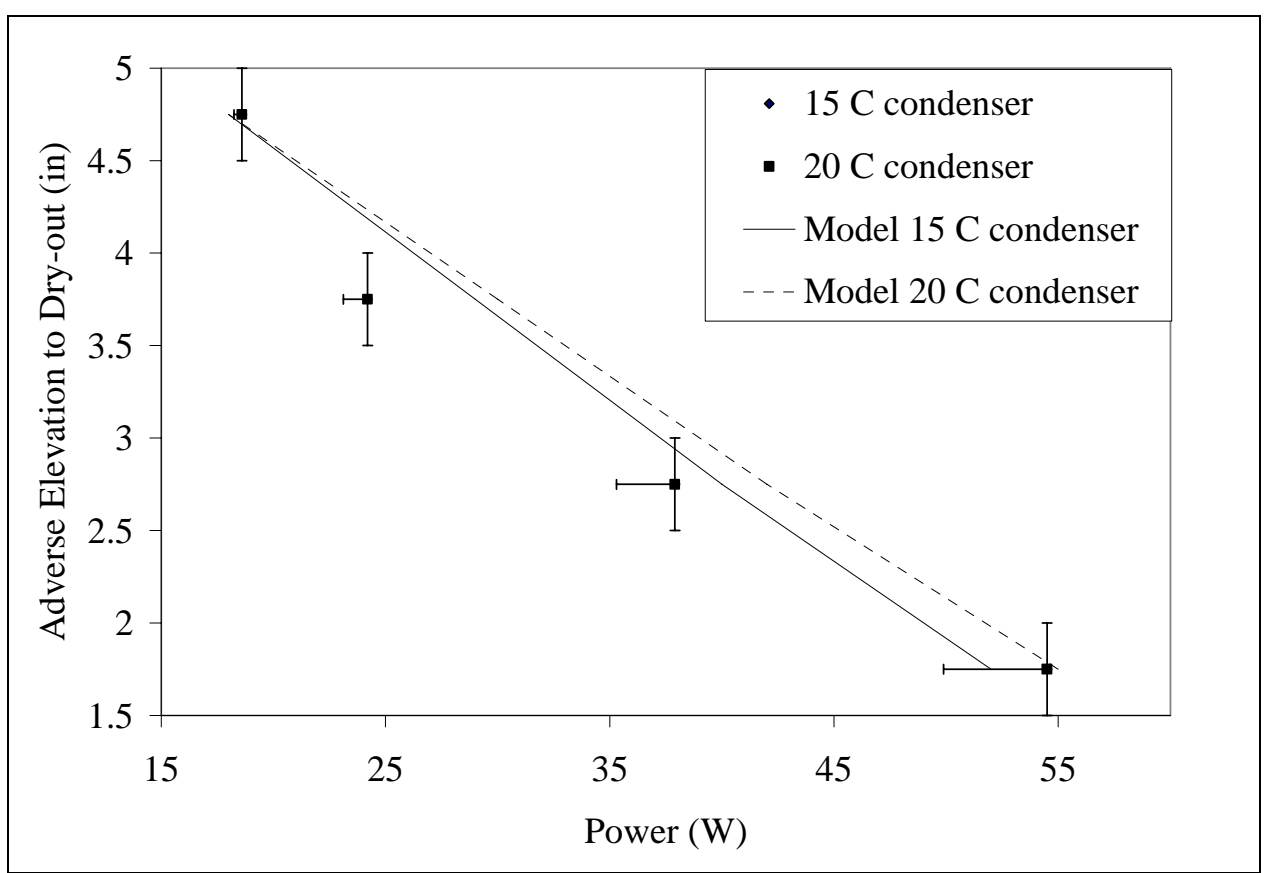

Figure 5.5. Stationary tests with the heat pipes installed in the rotating test apparatus.

the centrifugal force is helping with liquid return to the evaporator. The capillary limit, therefore, should increase with increasing rotation speed. Results of the low-speed test 
with a condenser set point of $25 \mathrm{C}$ and the heat pipe filled with $15.7 \mathrm{~g}$ of methanol are shown in Fig. 5.6. The data point for the 0 rpm case is taken with the heat pipe operating in $1 \mathrm{~g}$ gravity assist with the evaporator $24.1 \mathrm{~cm}$ below the condenser. The centrifugal acceleration, $\omega^{2} d_{\max }$ where $d_{\max }=24.1 \mathrm{~cm}$ is the distance from the axis of rotation to the off-axis evaporator section, increases as the rotation speed is increased. For $\omega^{2} d_{\max } / g<2$, the data indicate the capillary limit for the rotating heat pipe is less than the capillary limit for the non-rotating heat pipe with $1 g$ gravity assist. Recognizing that gravity is superimposed on the rotating heat pipe allows for an explanation of this behavior. At the top of the rotation cycle (with the evaporator above the condenser) and with $\omega^{2} d_{\max } / g<2$, the net acceleration assisting liquid return is less than $1 g$, resulting in a capillary limit lower than the $1 g$ gravity assist case. With the current experimental set-up, the capillary limit could not be found after the rotation speed was increased

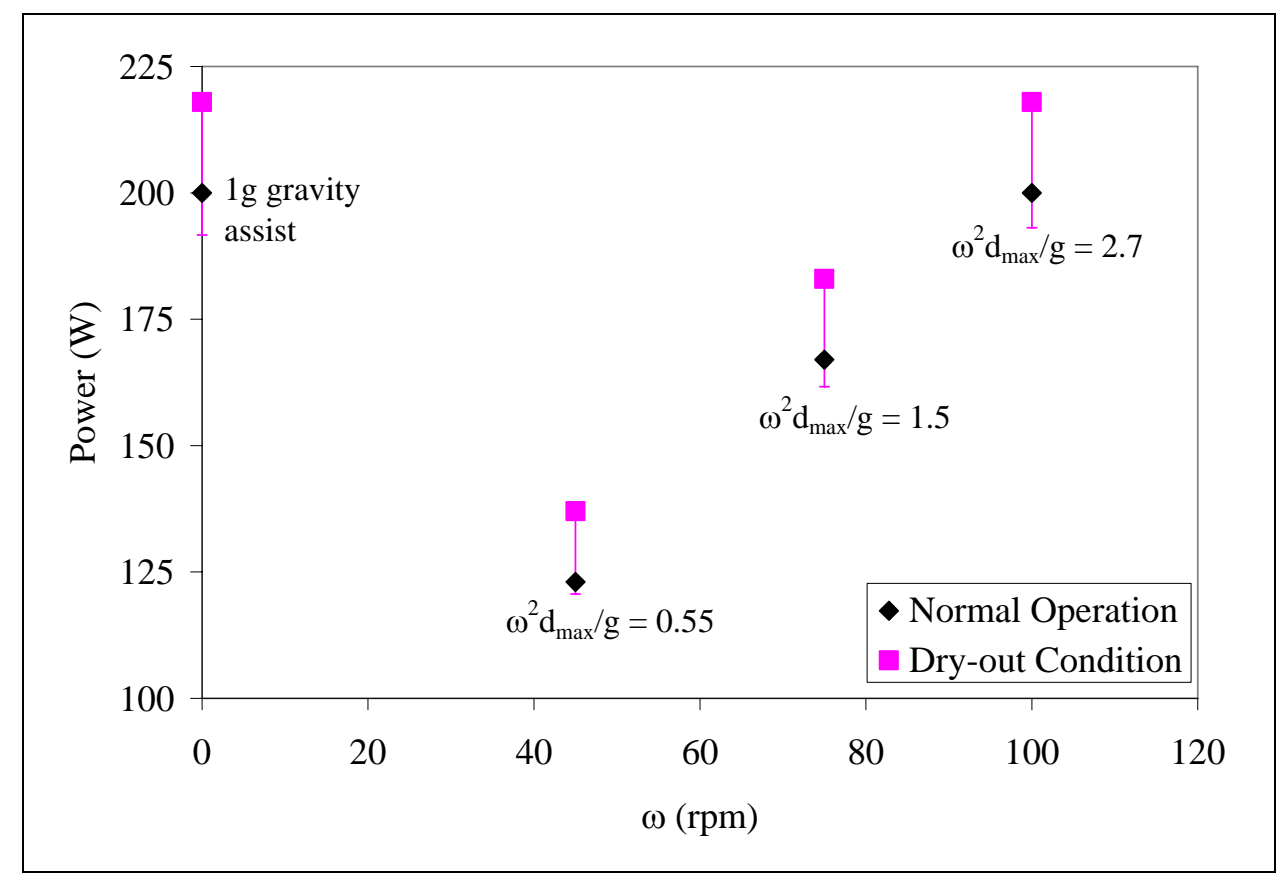

Figure 5.6. Low-speed dry-out tests. 
beyond $100 \mathrm{rpm}$ due to the limitation on the maximum power that could be supplied to the heat pipe.

\subsubsection{High Speed Test Data}

Although performance limits could not be determined at high rotational speeds, temperatures along the length of the heat pipe were measured while varying the rotational speed and heat load. For the heat pipe with a $15.7 \mathrm{~g}$ methanol charge, the rotational speed was varied from 210 to 1170 rpm, and the heat load was varied from 38 to $198 \mathrm{~W}$. An example data file is shown in Fig. 5.7. The figure shows that as the rotational speed is increased from 210 to $550 \mathrm{rpm}$, the temperatures in the adiabatic and evaporator sections are decreasing. This temperature decrease indicates that the excess liquid, which in a non-rotating horizontal heat pipe is swept to the condenser section by the counterflowing vapor, is being redistributed into the evaporator by the centrifugal force leading to a tighter coupling of the condenser section to the heat sink.

The measured temperature difference across the liquid film in the evaporator section (TC3 - TC4) for the heat pipe rotating up to $1170 \mathrm{rpm}$ is shown in Fig. 5.8. The figure shows that the temperature difference across the liquid film in the evaporator increases with rotation speed at low $(<420 \mathrm{rpm})$ speed, then decreases as the rotation speed is increased. The data from these tests with increasing heater power are collapsed to a single curve, shown in Fig. 5.9, by evaluating the thermal conductance ( $G=Q / \Delta T$ ) across the liquid film. As the temperatures differences in Fig. 5.8 would suggest, at low rotation speed, the conductance decreases with increasing rotation speed. The decrease in thermal conductance across the liquid film in the evaporator is caused by the redistribution of liquid in the heat pipe due to the centrifugal force. As the excess liquid 


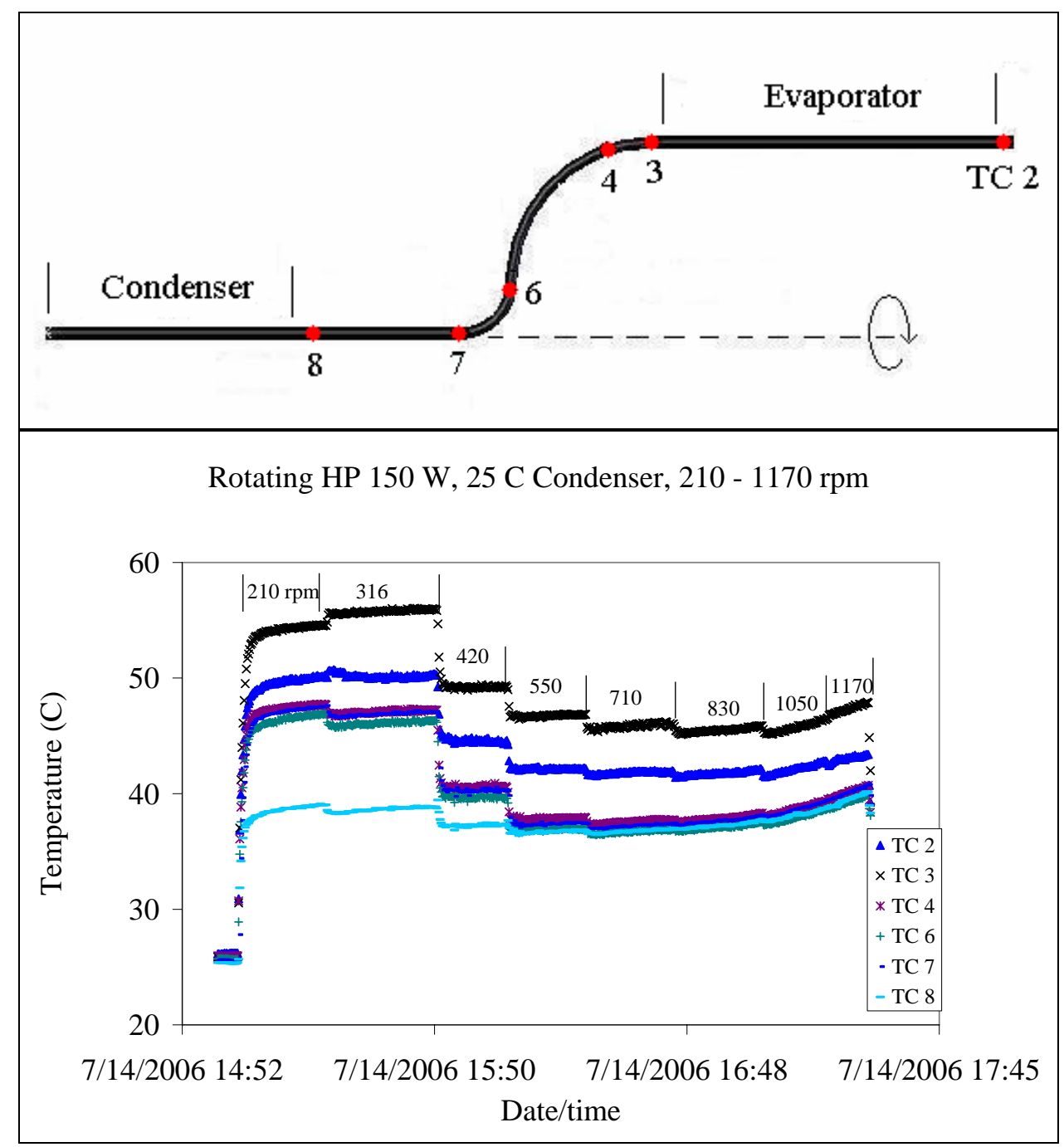

Figure 5.7. Example data file for a rotating test with a $150 \mathrm{~W}$ heat load and a condenser temperature of $25 \mathrm{C}$.

charge drains from the condenser into the evaporator, the liquid film in the evaporator gets thicker, decreasing the conductance. Once the rotation speed (and centrifugal force) is large enough to force all of the excess liquid into the evaporator ( $>420 \mathrm{rpm}$ ), further increases in rotation speed result in an increased thermal conductance across the liquid film. The increase in thermal conductance is likely due to an increase in the Nusselt number in the liquid film as the centrifugal force and Rayleigh number are increased.

From the conductance values shown in Fig. 5.9, the scaling between Nusselt number and Rayleigh number for the liquid film in the evaporator can be approximated. 


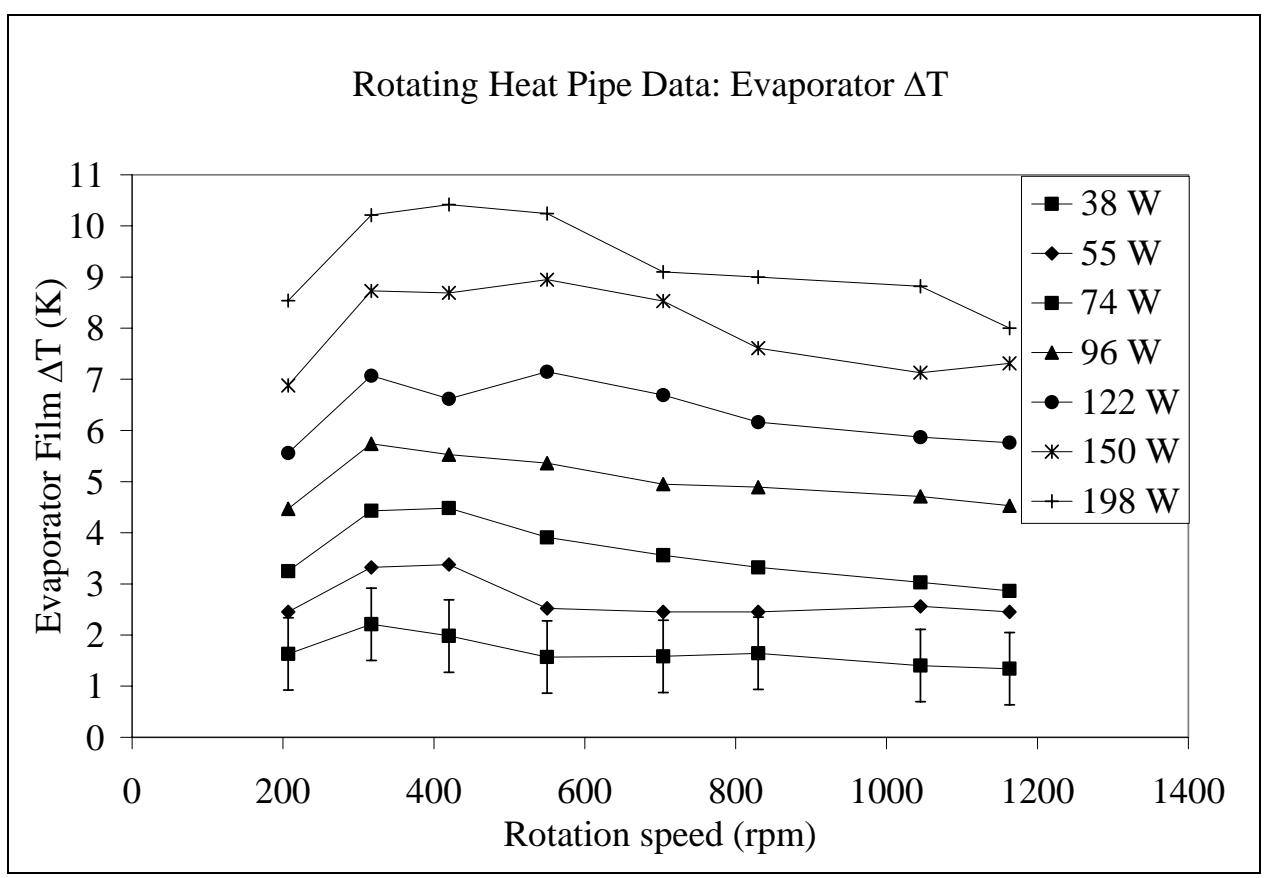

Figure 5.8. Measured temperature difference across the evaporator liquid film for the rotating tests. All uncertainties are the same as the $38 \mathrm{~W}$ case.

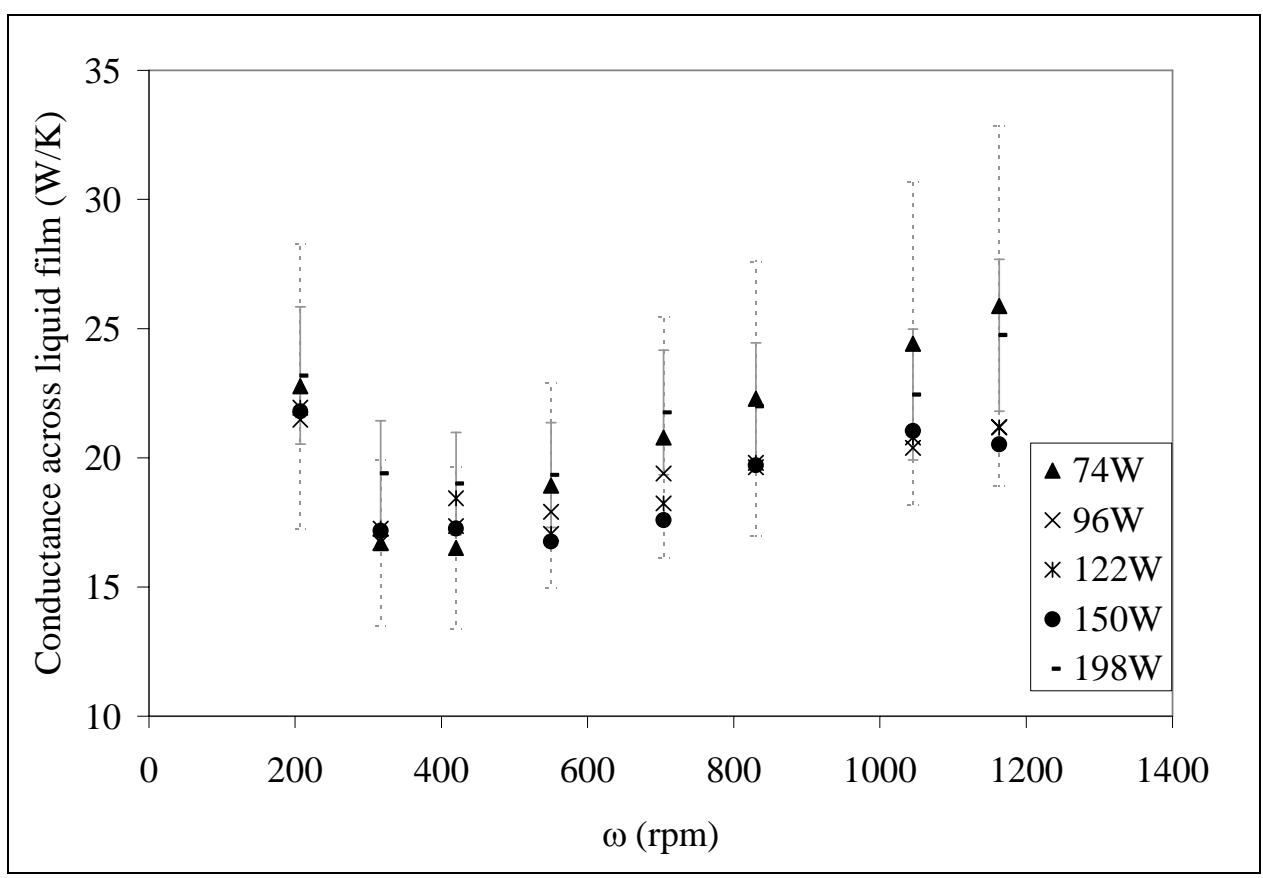

Figure 5.9. Conductance across the liquid film in the evaporator section of the rotating heat pipe. The $74 \mathrm{~W}$ case gave the largest (dashed) error bar and the 198 W case gave the smallest (solid) error bar when calculating the conductance.

Assuming that the fluid properties are constant, the Rayleigh number increases as $\omega^{2} \Delta T$.

Taking $\left(\omega^{2} \Delta T\right)_{\min }$ and $G_{\min }$ as the values from the $420 \mathrm{rpm}$ data points, the ratios 
$G / G_{\min }$ and $\left(\omega^{2} \Delta T\right) /\left(\omega^{2} \Delta T\right)_{\min }$ are representative of $N u / N u_{\min }$ and $R a / R a_{\min }$, respectively, for the data points with a rotation speed greater than $420 \mathrm{rpm}$. This relationship is shown in Fig. 5.10. The figure indicates that the exponent in power law relationship is 0.2. A curve fit of the correlation of Jankowski et al. given in Eq. (3.43) for $4,000 \leq R a \leq 10,000$ gives an exponent of 0.23 . This observation would suggest that heat transfer across the liquid film is influenced by buoyancy driven convection.

\subsection{Comparisons to the Rotating Heat Pipe Model}

The rotating heat pipe data and model predictions are compared in Figs. 5.11 and 5.12. The error bars on the numerical solutions are the GCI for the $102 \times 22$ grid used to calculate the data points in the figures. For this grid, the GCI was calculated as $6 \%$. Results from the numerical model using the Nusselt number correlation of Jankowski et al. [Eq. (3.43)] are shown in Fig. 5.11, while in Fig. 5.12 the correlation of Körner is used. Both simulations qualitatively capture the behavior of the heat pipe. In particular,

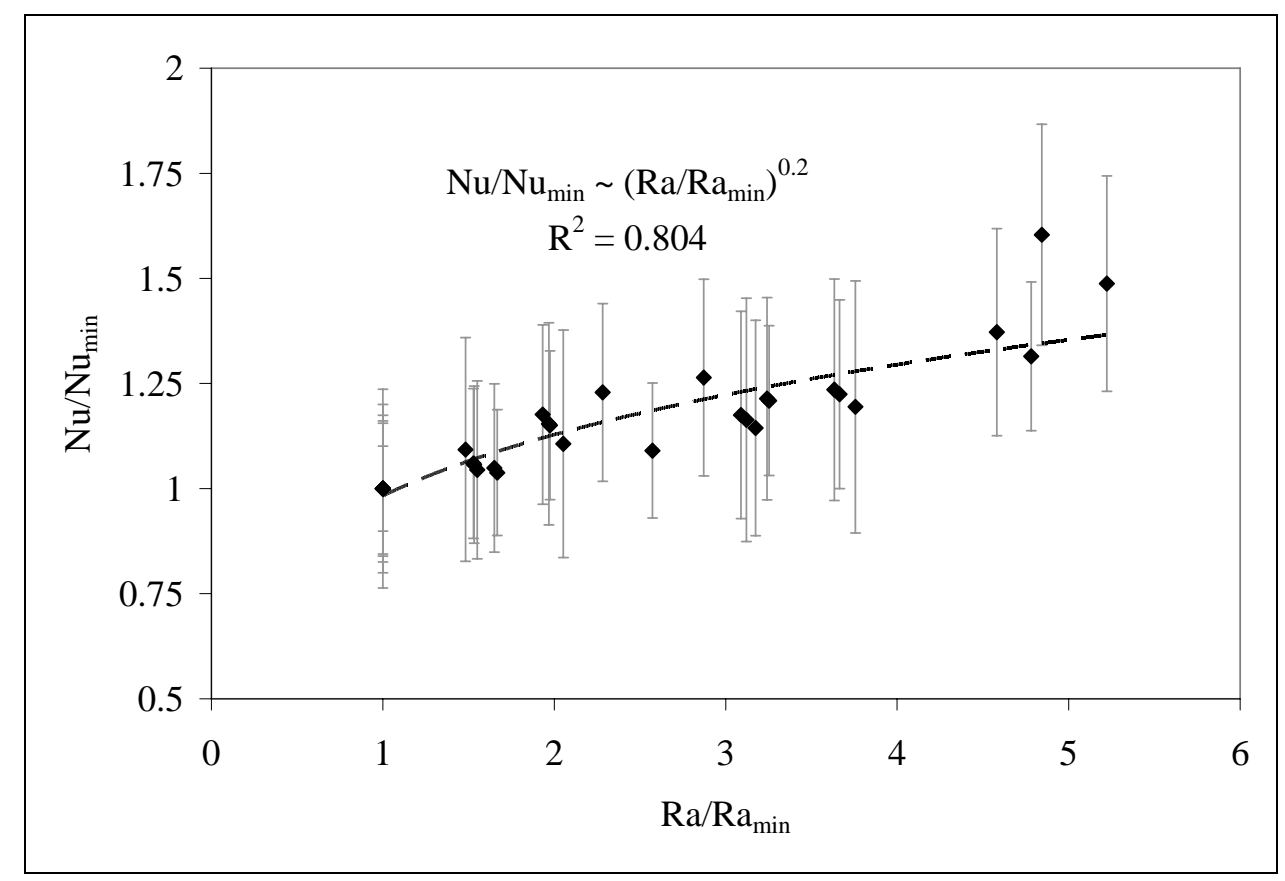

Figure 5.10. Nusselt number for heat transfer across the evaporator film in the rotating heat pipe. 


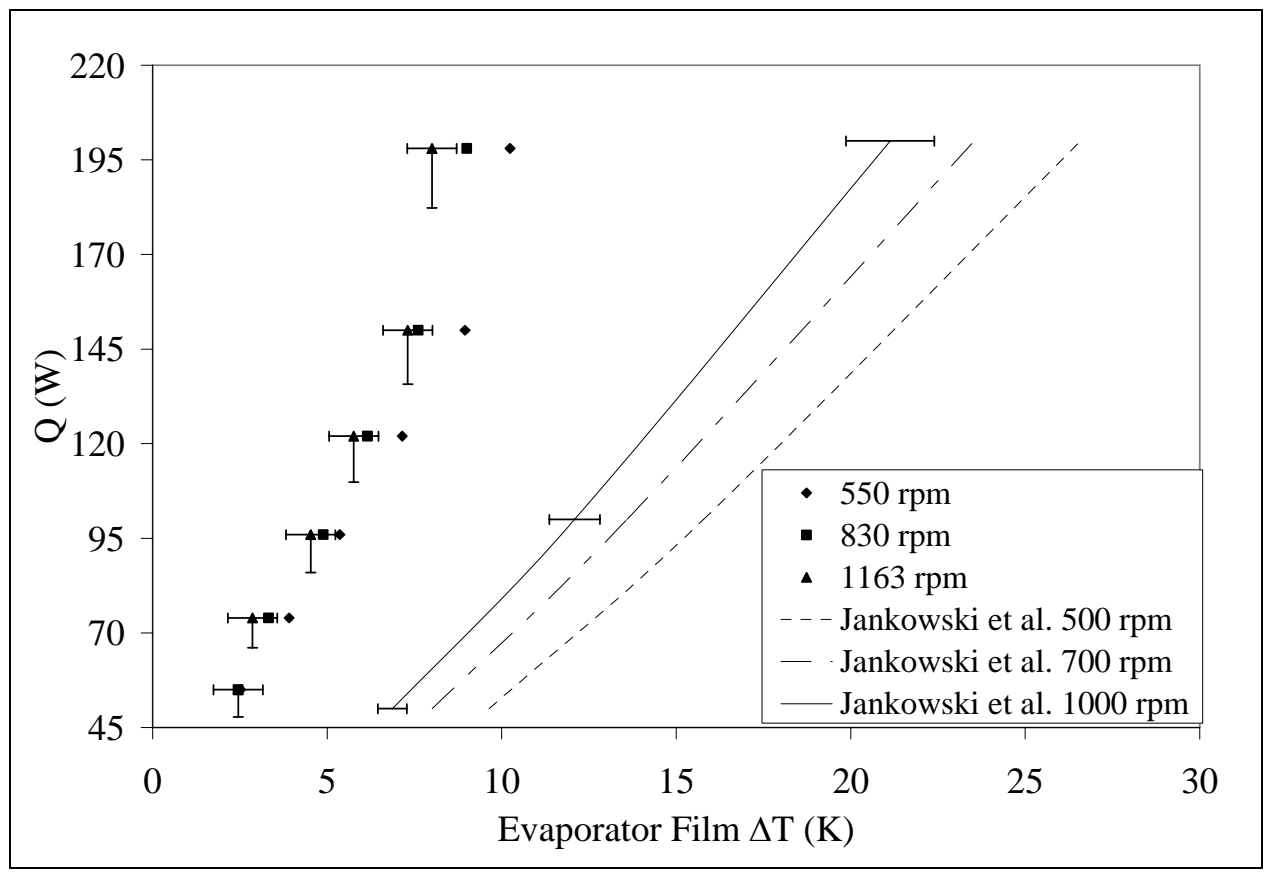

Figure 5.11. Comparing experimental results to the model using the convection correlation of Jankowski et al.

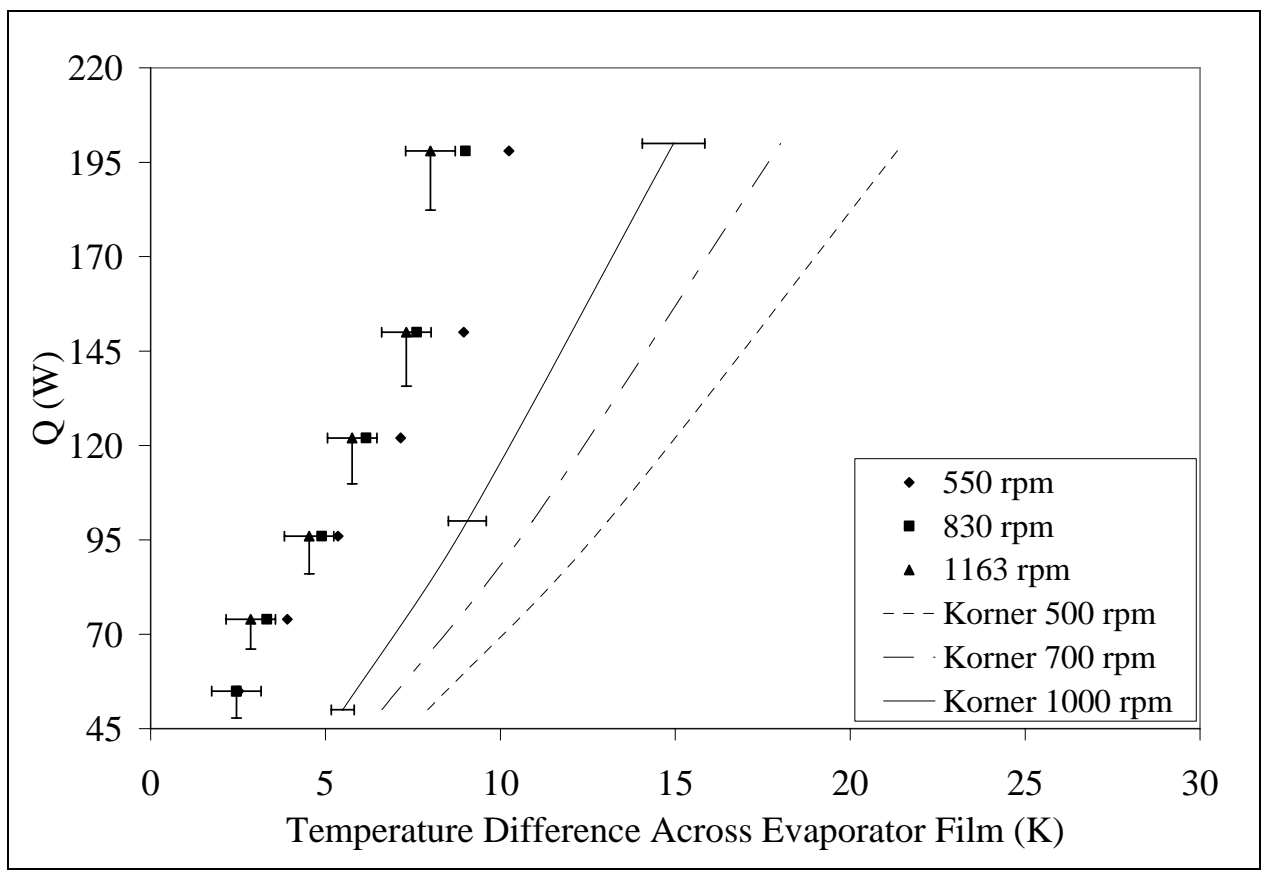

Figure 5.12. Comparing experimental results to the model using the convection correlation of Körner.

both model and experimental results suggest that increases in rotation speed and decreases in heat transfer rate reduce the temperature difference across the liquid film in the evaporator section. Additionally, the slopes of the curves generated by the model are 
close to those observed in the experiments, suggesting that the model is properly predicting the transition from wicked to non-wicked operating regimes and the associated redistribution of the working fluid. The model results, however, are overly conservative (that is, predicting higher temperature differences across the film for a given heat transfer rate). Using the correlation of Jankowski et al., the model over-predicts the temperature difference by as much as $160 \%$, whereas with the correlation of Körner, the model overpredicts the temperature difference by as much as $110 \%$.

The comparisons shown in Figs. 5.11 and 5.12 indicate that the model is predicting lower heat transfer coefficients across the liquid film in the evaporator than are observed in the experiments. In the development of the numerical model, the liquid flow was assumed laminar and two-dimensional (symmetry about the longitudinal axis). Additionally, the effects of mixed convection, due to the liquid flow from the condenser to the evaporator, on the heat transfer coefficient in the evaporator were ignored. These simplifying assumptions may contribute to the low estimates of the heat transfer coefficient in the evaporator section produced by the model.

\subsubsection{Mixed Convection and Turbulence in the Liquid}

When calculating the heat transfer coefficient in the evaporator, the heat pipe model ignores the presence of mixed convection in the liquid film by using a Nusselt number correlation for pure natural convection. A considerable amount of work has been done to assess the effect of buoyancy on forced convection between horizontal parallel plates (Osborne and Incropera 1985a; Osborne and Incropera 1985b) and in horizontal tubes (Hieber 1982; Nagendra 1973). Nusselt numbers for these mixed convection cases are 
calculated from the well-known formula for mixed convection in general (Churchill 1977)

$$
N u^{n}=N u_{F}^{n}+N u_{N}^{n},
$$

where subscripts $F$ and $N$ represent Nusselt numbers for pure forced or natural convection, respectively, and $n=3$ is suggested to provide a best fit to the experimental data (Hieber 1982; Osborne and Incropera 1985b). The relative importance of buoyancy to forced convection is assessed with the ratio $\mathrm{Gr} / \mathrm{Re}^{2}$. With the small film Reynolds numbers developed in the heat pipes studied here (see Fig. 3.16), $G r / \operatorname{Re}^{2}>>1$ in the evaporator, and natural convection in the liquid film will dominate. In this situation, the forced convection component in Eq. (5.1) can be ignored.

Although the heat transfer coefficient in the evaporator can be calculated from a natural convection correlation, the transition to thermal turbulence in the heated liquid film may affect the dynamics of the liquid flow. As discussed in Section 3.6.1 and illustrated in Fig. 3.16, typical Reynolds numbers in the liquid film are well below the transition value to induce hydrodynamic turbulence. However, as the Rayleigh number in the liquid film increases, the flow can transition due to thermal turbulence (Nagendra 1973). When calculating the liquid film thickness in the evaporator, the heat pipe model assumes a laminar liquid flow. If thermal effects are significant enough to develop a turbulent flow in the evaporator, the velocity profile and liquid layer thickness will differ from the values calculated by the laminar flow model.

\subsubsection{Three-Dimensional Pool Flow}

The model assumes that the liquid flow is two-dimensional, with the liquid forming a constant thickness annulus around the circumference of the heat pipe. In the experiment, 
however, the centrifugal force is large enough to overcome the circumferential pumping force of the porous wick (Semena et al. 1991). Therefore, the liquid will be forced to pool into a crescent shaped liquid layer on the outer wall, and only a portion of the circumference of the evaporator section will be covered with liquid. This threedimensional behavior has not been captured in the numerical model and may explain the discrepancies observed when comparing predictions to the experiments.

When the liquid is forced to pool on the surface of the evaporator, the evaporator section is behaving as a revolving heat pipe. Only a limited number of studies devoted to the heat transfer in revolving heat pipes have been carried out (Curtila and Chataing 1984; Gi and Maezawa 1990; Niekawa et al. 1981; Pokorny et al. 1984). All of these studies had an experimental component, but only Curtila and Chataing attempted to characterize the performance of their revolving heat pipe with a heat pipe model based on a thermal resistance network (Curtila and Chataing 1984). In the model by Curtila and Chataing, the thermal resistance in the evaporator section of the revolving heat pipe is crudely estimated by assuming that half of the evaporator surface is covered by liquid with a boiling heat transfer coefficient of $2000 \mathrm{~W} / \mathrm{m}^{2} \mathrm{~K}$. The heat transfer coefficient over the entire evaporator surface area is then estimated as $1000 \mathrm{~W} / \mathrm{m}^{2} \mathrm{~K}$.

In Fig. 5.13, the measured heat transfer coefficient across the evaporator film from the heat pipe tested here is compared to the measured heat transfer coefficient reported by Gi and Maezawa (Gi and Maezawa 1990). The calculated heat transfer coefficient of Curtila and Chataing and the heat transfer coefficient calculated with the present model which assumes that the liquid is evenly distributed around the circumference are also shown on the figure. The figure clearly shows that the present model is underestimating 


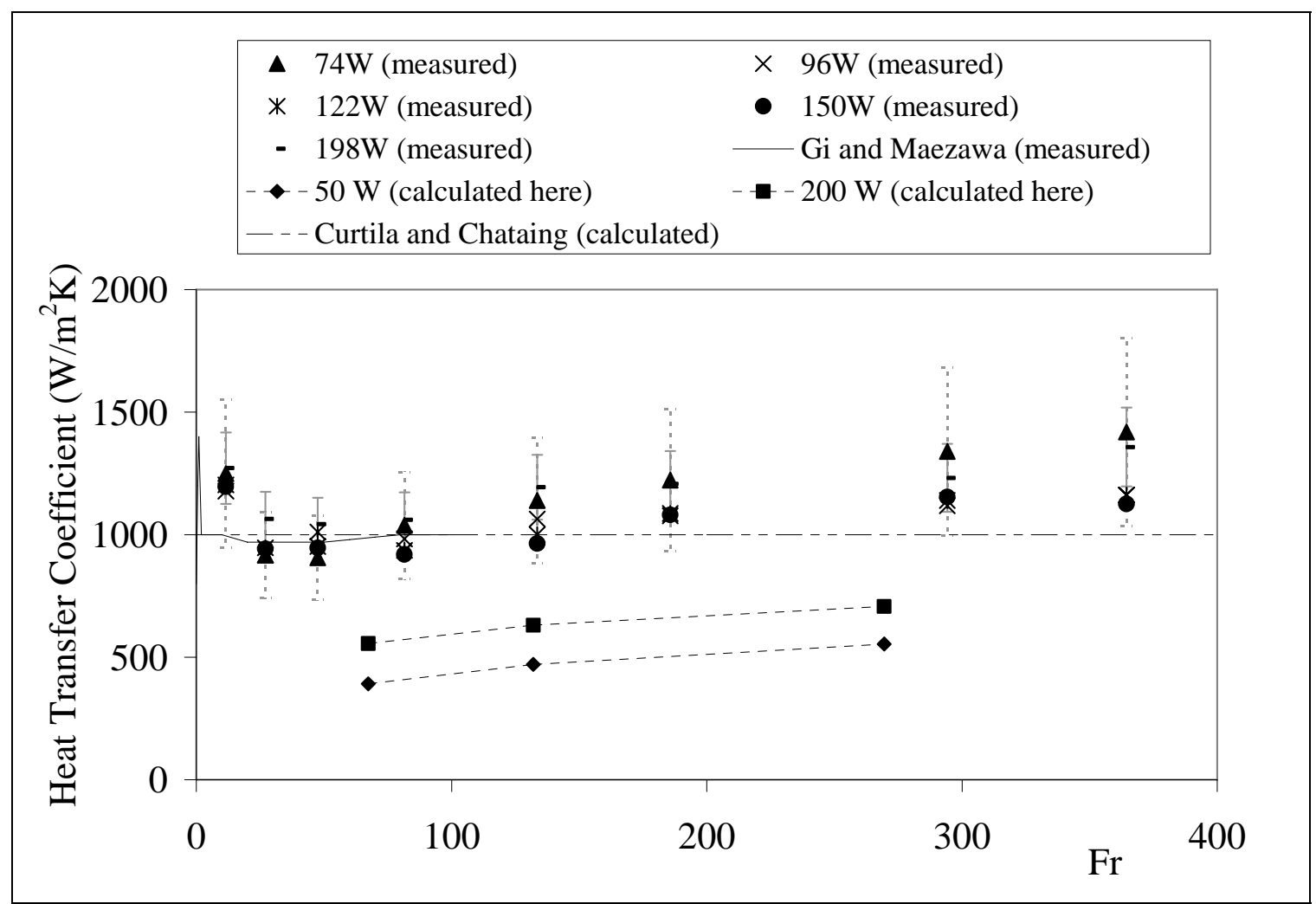

Figure 5.13. Comparing heat transfer coefficients in the evaporator. The dashed error bars are for the $74 \mathrm{~W}$ data and the $198 \mathrm{~W}$ error bars are solid.

the heat transfer coefficient in evaporator section, resulting in the high estimates of the temperature difference across the evaporator film shown in Figs. 5.11 and 5.12. Although the simple correlation provided by Curtila and Chataing does agree with both the experimental data collected here and the data of Gi and Maezawa, it does not account for the variations in heat transfer coefficient with rotation speed, fluid charge, working fluid properties, and heat pipe geometry.

Observations from experiments with rotating (on-axis) heat pipes at low-speed also indicate that the annular flow assumption used here would underestimate the heat transfer coefficient when the liquid forms a pool in the evaporator section (Jian et al. 1987; Jian et al. 1990; Krivosheev et al. 1979; Reddy et al. 1987; Reddy et al. 1990). As discussed in Sec. 2.3.1, when the liquid in the rotating heat pipe transitions from pool flow to annular 
flow, the heat transfer coefficients in the condenser and evaporator decrease by typically an order of magnitude. The numerical model developed here assumes that, regardless of the rotation speed, the liquid in the evaporator forms a continuous annulus around the circumference of the heat pipe. Although heat transfer coefficients in the pool flow regime have been measured, no correlations or theories are available to use in a design calculation.

The heat transfer coefficient in the evaporator section may also be enhanced (over the values observed in smooth-walled tubes) by the presence of the wick. Experimental investigations have found that shallow grooves on the inside wall of revolving heat pipes tend to spread the liquid over a larger area and increase the heat transfer coefficient (Castle et al. 2001; Klasing et al. 1999; Niekawa et al. 1981). The screen wick pressed against the wall by the centrifugal force may have the same effect as a grooved wall.

It is clear from the comparisons of the numerical and experimental results that more work is needed to characterize and quantify the heat transfer in revolving heat pipes and rotating heat pipes operating in the pool flow regime. The limited data available, however, does seem to confirm the presumption that the differences between the calculated and observed performance for the heat pipe studied here are in large part due to the three-dimensional nature of the flow in the revolving evaporator section. 


\section{Concluding Remarks}

\subsection{SUMMARY}

A unique heat pipe for off-axis cooling of rotating machinery has been studied both numerically and experimentally. The heat pipe is built so that the condenser section is an on-axis rotating section, the evaporator section is off-axis and revolving, and the adiabatic section contains an S-shaped curve which joins the condenser and evaporator. This heat pipe geometry could find use in cooling rotating devices with off-axis heat sources (in contact with the evaporator) and where coupling to a heat sink (at the condenser section) is most easily and efficiently achieved on-axis. The heat pipe studied here also contains an annular gap screen wick structure allowing for operation when the heat pipe is not rotating.

A numerical model of the rotating heat pipe has been developed. The model is based on a two-dimensional (symmetry about the longitudinal axis) finite-difference model of the liquid flow coupled to a one-dimensional model of the vapor flow. The model can predict the performance of rotating heat pipes without a wick structure as well as heat pipes with an annular gap wick.

The model developed here represents an extension to previous analyses of the liquid flow in wickless rotating heat pipes which are based on a Nusselt-type film flow analysis. In the Nusselt-type film flow analysis, simplified forms of the governing equations are applied to the liquid to determine the liquid layer thickness along the wall of the heat pipe. Rather than solving simplified versions of the governing equations, the present heat pipe model solves the full discretized Navier-Stokes and energy equations, subject to the associated free-surface boundary conditions, in the liquid layer. In the analysis, the liquid 
and vapor flows are treated separately, and a coordinate transformation and mapping procedure is used to determine the varying location of the liquid-vapor interface in nonwicked operation. The coordinate transformation and the method used for application of the free-surface boundary conditions have been extensively used and tested in previous investigations of free-surface flows (Faghri et al. 1984; Pacheco and Peck 2000; Sim et al. 2004).

Treating the liquid and vapor flow domains separately and coupling them through the boundary conditions at the interface has distinct advantages over a single-domain approach suggested previously for the analysis of rotating heat pipes (Baker et al. 1999). In particular, in the code developed here, a simplified model of the vapor flow can be used when the overall performance of the heat pipe is not affected by the dynamics of the vapor flow. Although temperature and pressure variations in the vapor phase of most rotating heat pipes have a negligible impact on the overall thermal performance (Song et al. 2003), the model developed here for the flow in the liquid could be coupled to more detailed models of the vapor flow if the need were to arise.

For the results presented throughout this work, discretization errors have been assessed and minimized using the GCI method (Roache 1998). The code has been validated by modeling a stationary (non-rotating) heat pipe and comparing the results to another extensively benchmarked heat pipe design code (Woloshun et al. 1988). Additionally, rotating wickless heat pipes were modeled and the results were compared to previous analyses (Daniels and Al-Jumaily 1975; Song et al. 2003).

The code developed here was used to model the heat pipe with the S-shaped curve. The model shows that in non-rotating operation and at low-speed, the liquid fills the 
annular gap wick, and the capillary pressure generated is used for liquid return to the evaporator. As the rotation speed is increased a critical rotation speed is reached, after which, the centrifugal forces acting on the working fluid cause the liquid to recede from the wick and form a variable thickness film along the wall of the adiabatic and evaporator sections. This transition from operating as a wicked heat pipe to a heat pipe which does not rely on the wick is behavior that could not be explained by the existing Nusselt-type analyses and has not been previously reported. Predicting the performance of this unique heat pipe requires an analysis that includes the liquid acceleration terms and the presence of the wick.

Using the analysis code developed here, parametric studies were performed for the heat pipe with the S-shaped curve. These studies assessed the impacts of varying the heat load, rotation speed, geometry, and fluid charge on the heat pipe performance. The parametric studies showed that the liquid is confined to the annular gap wick structure in the condenser section and that the thermal resistance in the condenser is dominated by conduction across the liquid layer. Additionally, the saturated vapor experiences a pressure (and temperature) rise in the S-shaped curve that is determined by the hydrostatic pressure required to overcome the centrifugal forces. Finally, the parametric studies showed that the heat transfer in the off-axis evaporator section is influenced by natural convection in the heated liquid layer.

A literature survey (see Appendix B) showed that the empirical correlations suggested for natural convection and boiling in evaporating liquid layers are not applicable to most rotating heat pipes. Steady laminar natural convection in an 
evaporating liquid layer was modeled numerically and an appropriate Nusselt number correlation was developed for typical heat pipe operating conditions.

Experiments were performed to characterize the heat transfer performance of the rotating heat pipe incorporating the S-shaped curve. A method of fabricating the long curved heat pipe has been developed. Stationary performance tests were conducted to verify that the wick structure was not damaged during fabrication or bending.

A rotating apparatus for testing heat pipes incorporating the S-shaped curve has been designed and built. Rotating tests were performed with the heat pipe at speeds up to 1200 rpm and carrying heat loads up to $200 \mathrm{~W}$. The test data indicate a redistribution of the liquid in the heat pipe at low $(<500 \mathrm{rpm})$ speed, and that excess liquid is forced into the evaporator section. Heat transfer across the liquid film in the evaporator is shown to be influenced by buoyancy driven convection. The test data indicate that the temperature difference required to transfer heat decreases as the rotation speed is increased and as the heat transfer rate is decreased. All of these observations are qualitatively captured by the numerical model.

\subsection{SUgGeSTIONS FOR FUTURE WORK}

The numerical model developed here does include many aspects of the flow in the rotating heat pipe and suggests a unique type of behavior (the transition from the use of the wick to the wickless operation) for the heat pipe with the S-shaped curve. When comparing the results of the numerical model to the experiments, however, the numerical model is predicting temperature differences across the evaporator film that are larger than those observed in the experiments. The two-dimensional assumption, particularly in the 
evaporator section where the liquid will collect on the outermost region of the tube wall, does not accurately capture the heat transfer process in the evaporator.

Previous experimental studies of low-speed rotating heat pipes and revolving heat pipes suggest that heat transfer coefficients in the evaporator sections of heat pipes with a large liquid pool are considerably higher than in heat pipes with a uniformly distributed liquid annulus. Although there is experimental evidence to suggest that there are significant differences between heat transfer in the pool flow and annular flow regimes, no correlations are available to predict heat transfer coefficients in heat pipes with a liquid pool. A modeling study, dedicated to the heat transfer in a revolving heat pipe evaporator, may be a worthwhile first step toward understanding this process.

The numerical model developed here could be extended to model the vapor flow in more detail in rotating wickless heat pipes, and couple this detailed vapor flow model to the liquid flow model developed here. Although finite difference vapor flow models have been developed for axially rotating heat pipes (Faghri et al. 1993), these models have either been coupled to the liquid confined to a homogeneous wick (Ismail and Miranda 1997; Machado and Miranda 2003) or to a simplified Nusselt-type analysis in the liquid layer (Harley and Faghri 1995). By including all of the terms in the freesurface boundary conditions for non-wicked operation, the liquid flow model developed here, coupled to a finite-difference model of the vapor flow, may provide a deeper understanding of the interaction between the liquid and vapor flows in axially rotating wickless heat pipes. 


\section{LIST OF APPENDICES}

Appendix A: SIMPLER

Appendix B: Heat Transfer in an Evaporating Liquid Layer

Appendix C: Experimental Uncertainty Analysis 


\section{APPENDIX A: SIMPLER}

This section summarizes the sequence of steps in the numerical marching procedure used in the heat pipe design code described in Chapter 3. The solution of the governing equations follows the Semi-implicit Method for Pressure Linked Equations-Revised (SIMPLER) applied to parabolic flows. Details of SIMPLE and its revised version (SIMPLER), applied to the fully-elliptic Navier-Stokes equations are described in the book by Patankar (Patankar 1980). The application of SIMPLE to parabolic flows is described by Patankar and Spalding (Patankar and Spalding 1972). The terminology used here follows that of the original references.

The continuity, momentum, and energy equations, given in Eqs.(3.27) through (3.32), are integrated over the control volumes shown on the four point finite-difference stencil in Fig. A.1. As was mentioned in Chapter 3, $\Pi, \Lambda, u_{\theta}$, and $u_{x}$ are stored at the grid nodes $(i, j)$, and $u_{\xi}$ is stored on the face of the control volume surrounding each node $(i, j+1 / 2)$. During the integrations, second-order centered differences are used to approximate the diffusion terms in the cross-stream ( $\xi$ ) direction, the QUICK method

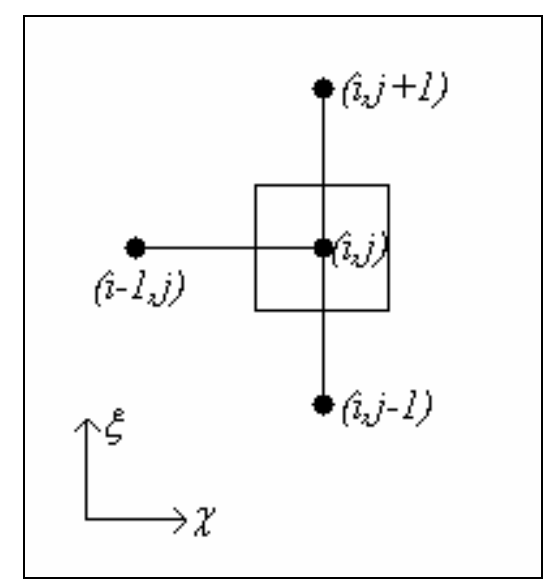

Figure A.1. The finite-difference stencil used for integrating the equations. 
(Leonard 1979) is used for the convective terms in the $\xi$-direction, and first-order backward-differences approximate the convective terms in the $\chi$-direction.

The algebraic equations resulting from the discretization are cast into a nominally linear form at a given axial location $(i)$ :

$$
\begin{array}{lc}
\text { continuity: } & C_{N} u_{\xi(i, j+1 / 2)}-C_{S} u_{\xi(i, j-1 / 2)}+B_{c}=0 \\
\xi \text {-mom.: } A_{p}^{u_{\xi}} u_{\xi(i, j+1 / 2)}=A_{S}^{u_{\xi}} u_{\xi(i, j-1 / 2)}+A_{N}^{u_{\xi}} u_{\xi(i, j+3 / 2)}+A_{\Pi}^{u_{\xi}}\left(\Pi_{(i, j)}-\Pi_{(i, j+1)}\right)+B^{u_{\xi}} \\
\theta \text {-mom.: } \\
\quad A_{p}^{u_{\theta}} u_{\theta(i, j)}=A_{S}^{u_{\theta}} u_{\theta(i, j-1)}+A_{N}^{u_{\theta}} u_{\theta(i, j+1)}+B^{u_{\theta}} . \\
\text { x-mom.: } & A_{p}^{u_{x}} u_{x(i, j)}=A_{S}^{u_{x}} u_{x(i, j-1)}+A_{N}^{u_{x}} u_{x(i, j+1)}+A_{\Pi}^{u_{x}} \frac{\partial \bar{\Pi}}{\partial \chi}+B^{u_{x}} \\
\text { energy: } & A_{p}^{\Lambda} \Lambda_{(i, j)}=A_{S}^{\Lambda} \Lambda_{(i, j-1)}+A_{N}^{\Lambda} \Lambda_{(i, j+1)}+B^{\Lambda} .
\end{array}
$$

The terms $B^{\phi}$ contain the source terms from the momentum and energy equations. Additionally, the convective terms in the $\chi$-direction, which are discretized using backward-differences, are absorbed into the $B_{c}$ and $B^{\phi}$ terms. These convective terms allow the values of the dependent variables (velocity and temperature) from the previous axial location $(i-1)$ to be incorporated into the discrete equations at the current axial location ( $i)$.

The coefficients $A_{p}, A_{S}$, and $A_{N}$ result from a discretization of both the diffusion and convection terms in the momentum and energy equations. Because the convective terms are non-linear, the velocity appears in these coefficients. Absorbing the non-linear terms into the coefficients requires the linear equations to be solved iteratively at each axial location.

The steps used in the solution of Eqs. (A.1) through (A.5) are: 
1. Starting at $\chi=0$, guess the values of velocity and temperature at $\chi=\Delta \chi$.

2. Calculate the $A, B$, and $C$ coefficients in Eqs. (A.1) through (A.5).

3. Calculate the pseudo-velocities $\left(\hat{u}_{\xi}\right.$ and $\hat{u}_{x}$ ) and the pseudo-mass source $\left(\hat{m}_{n}\right)$.

4. Solve for the first approximation to the pressure $\left(\Pi^{*}\right)$.

5. Use the global mass balance to obtain a first approximation to the averaged pressure gradient $\left(\partial \bar{\Pi}^{*} / \partial \chi\right)$.

6. Solve the momentum equations for the first approximations to the velocity components $\left(u_{\xi}^{*}, u_{\theta}\right.$, and $\left.u_{x}^{*}\right)$ using the pressure and pressure gradient from steps 4 and 5 .

7. Calculate the mass source $\left(m_{n}\right)$ and solve for the pressure correction $\left(\Pi^{\prime}\right)$.

8. Use the global mass balance to calculate the pressure gradient correction $\left(\partial \bar{\Pi}^{\prime} / \partial \chi\right)$

9. Using the pressure and pressure gradient corrections, correct the velocities to give $u_{\xi}$ and $u_{x}$.

10. Solve the energy equation for the temperature $\Lambda$.

11. Iterate steps 2-10 until convergence is achieved.

12. March to the next $\chi$ location and repeat steps 1-11.

The details in each step of the calculation procedure are:

Step 3: The pseudo-velocities are defined as

$$
\hat{u}_{\xi(i, j+1 / 2)}=\frac{A_{S}^{u_{\xi}}}{A_{p}^{u_{\xi}}} u_{\xi(i, j-1 / 2)}+\frac{A_{N}^{u_{\xi}}}{A_{p}^{u_{\xi}}} u_{\xi(i, j+3 / 2)}+\frac{B^{u_{\xi}}}{A_{p}^{u_{\xi}}},
$$




$$
\hat{u}_{x(i, j)}=\frac{A_{S}^{u_{x}}}{A_{p}^{u_{x}}} u_{x(i, j-1)}+\frac{A_{N}^{u_{x}}}{A_{p}^{u_{x}}} u_{x(i, j+1)}+\frac{B^{u_{x}}}{A_{p}^{u_{x}}} .
$$

From the continuity equation, the pseudo-mass source is then written as

$$
\hat{m}_{n}=C_{N} \hat{u}_{\xi(i, j+1 / 2)}-C_{S} \hat{u}_{\xi(i, j-1 / 2)}+B_{c} .
$$

Step 4: Equation (A.6) allows the velocity in the $\xi$-direction to be written as

$$
u_{\xi(i, j+1 / 2)}=\hat{u}_{\xi(i, j+1 / 2)}+\frac{A_{\Pi}^{u_{\xi}}}{A_{p}^{u_{\xi}}}\left(\Pi_{(i, j)}^{*}-\Pi_{(i, j+1)}^{*}\right) .
$$

Substituting Eq. (A.9) into the discrete form of the continuity equation and using Eq. (A.8) shows that

$$
\left[C_{N}\left(\frac{A_{1}^{u_{\xi}}}{A_{p}^{u_{\xi}}}\right)_{n}+C_{S}\left(\frac{A_{1}^{u_{\xi}}}{A_{p}^{u_{\xi}}}\right)_{s}\right] \Pi_{(i, j)}^{*}=C_{N}\left(\frac{A_{1}^{u_{\xi}}}{A_{p}^{u_{\xi}}}\right)_{n} \Pi_{(i, j+1)}^{*}+C_{S}\left(\frac{A_{1}^{u_{\xi}}}{A_{p}^{u_{\xi}}}\right)_{s} \Pi_{(i, j-1)}^{*}-\hat{m}_{n},(
$$

where the terms $\left(A_{\Pi \Pi}^{u_{\xi}} / A_{p}^{u_{\xi}}\right)_{n}$ and $\left(A_{\Pi}^{u_{\xi}} / A_{p}^{u_{\xi}}\right)_{s}$ are the coefficients $\left(A_{\Pi \Pi}^{u_{\xi}} / A_{p}^{u_{\xi}}\right)$ evaluated for control volumes centered around nodes $(i, j+1 / 2)$ and $(i, j-1 / 2)$, respectively. Equation (A.10) is solved for the pressure approximation.

Step 5: The definition of the pseudo-velocity in the $\chi$-direction allows the velocity to be written as

$$
u_{x(i, j)}=\hat{u}_{x(i, j)}+\frac{A_{\Pi}^{u_{x}}}{A_{p}^{u_{x}}} \frac{\partial \bar{\Pi}^{*}}{\partial \chi} .
$$

The expression for the mass flow rate in Eq. (3.59) is approximated as

$$
\frac{\bar{m}}{2 \pi \bar{\delta}}=\sum_{j}\left(\bar{R}_{i}+\xi_{j} \bar{\delta}_{i}-\bar{\delta}_{i}\right) u_{x(i, j)} \Delta \xi
$$

Substituting Eq. (A.11) into Eq. (A.12), and solving for the pressure gradient approximation gives 


$$
\frac{\partial \bar{\Pi}^{*}}{\partial \chi}=\frac{\frac{\bar{m}_{i}}{\left(2 \pi \bar{\delta}_{i}\right)}-\sum_{j}\left(\bar{R}_{i}+\xi_{j} \bar{\delta}_{i}-\bar{\delta}_{i}\right) \hat{u}_{x(i, j)} \Delta \xi}{\sum_{j}\left(\bar{R}_{i}+\xi_{j} \bar{\delta}_{i}-\bar{\delta}_{i}\right)\left(A_{\Pi}^{u_{x}} / A_{p}^{u_{x}}\right) \Delta \xi} .
$$

Steps 6 and 7: In Step 6, the first approximations for the velocities are obtained by solving

$$
\begin{gathered}
A_{p}^{u_{\xi}} u_{\xi(i, j+1 / 2)}^{*}=A_{S}^{u_{\xi}} u_{\xi(i, j-1 / 2)}^{*}+A_{N}^{u_{\xi}} u_{\xi(i, j+3 / 2)}^{*}+A_{\Pi}^{u_{\xi}}\left(\Pi_{(i, j)}^{*}-\Pi_{(i, j+1)}^{*}\right)+B^{u_{\xi}} \\
A_{p}^{u_{\theta}} u_{\theta(i, j)}=A_{S}^{u_{\theta}} u_{\theta(i, j-1)}+A_{N}^{u_{\theta}} u_{\theta(i, j+1)}+B^{u_{\theta}} . \\
A_{p}^{u_{x}} u_{x(i, j)}^{*}=A_{S}^{u_{x}} u_{x(i, j-1)}^{*}+A_{N}^{u_{x}} u_{x(i, j+1)}^{*}+A_{\Pi}^{u_{x}} \frac{\partial \bar{\Pi}^{*}}{\partial \chi}+B^{u_{x}}
\end{gathered}
$$

The velocity, pressure, and pressure gradient corrections are defined so that

$$
u_{\xi}=u_{\xi}^{*}+u_{\xi}^{\prime}, u_{x}=u_{x}^{*}+u_{x}^{\prime}, \Pi=\Pi^{*}+\Pi^{\prime} \text {, and } \frac{\partial \bar{\Pi}}{\partial \chi}=\frac{\partial \bar{\Pi}^{*}}{\partial \chi}+\frac{\partial \bar{\Pi}^{\prime}}{\partial \chi} .
$$

Subtracting Eqs. (A.14) and (A.16) from Eqs.(A.2) and (A.4) and using Eq. (A.17) gives

$$
\begin{gathered}
A_{p}^{u_{\xi}} u_{\xi(i, j+1 / 2)}^{\prime}=\left[A_{s}^{u_{\xi}} u_{\xi(i, j-1 / 2)}^{\prime}+A_{N}^{u_{\xi}} u_{\xi(i, j+3 / 2)}^{\prime}\right]+A_{\Pi}^{u_{\xi}}\left(\Pi_{(i, j)}^{\prime}-\Pi_{(i, j+1)}^{\prime}\right) \\
A_{p}^{u_{x}} u_{x(i, j)}^{\prime}=\left[A_{S}^{u_{x}} u_{x(i, j-1)}^{\prime}+A_{N}^{u_{x}} u_{x(i, j+1)}^{\prime}\right]+A_{\Pi}^{u_{x}} \frac{\partial \bar{\Pi}^{\prime}}{\partial \chi}
\end{gathered}
$$

Ignoring the terms in brackets gives the velocity corrections

$$
\begin{gathered}
u_{\xi(i, j+1 / 2)}^{\prime}=\frac{A_{\Pi !}^{u_{\xi}}}{A_{p}^{u_{\xi}}}\left(\Pi_{(i, j)}^{\prime}-\Pi_{(i, j+1)}^{\prime}\right) \\
u_{\chi(i, j)}^{\prime}=\frac{A_{1}^{u_{x}}}{A_{p}^{u_{x}}} \frac{\partial \bar{\Pi}^{\prime}}{\partial \chi} .
\end{gathered}
$$

Substituting Eq. (A.20) into the continuity equation and using Eq. (A.17) gives the equation that is solved for the pressure correction 


$$
\left[C_{N}\left(\frac{A_{\Pi}^{u_{\xi}}}{A_{p}^{u_{\xi}}}\right)_{n}+C_{S}\left(\frac{A_{\Pi}^{u_{\xi}}}{A_{p}^{u_{\xi}}}\right)_{s}\right] \Pi_{(i, j)}^{\prime}=C_{N}\left(\frac{A_{\Pi}^{u_{\xi}}}{A_{p}^{u_{\xi}}}\right)_{n} \Pi_{(i, j+1)}^{\prime}+C_{S}\left(\frac{A_{\Pi}^{u_{\xi}}}{A_{p}^{u_{\xi}}}\right)_{s} \Pi_{(i, j-1)}^{\prime}-m_{n},(\mathrm{~A} .22)
$$

with the mass source

$$
m_{n}=C_{N} u_{\xi(i, j+1 / 2)}^{*}-C_{S} u_{\xi(i, j-1 / 2)}^{*}+B_{c}
$$

Step 8: Using Eqs. (A.21) and (A.17), along with the global mass balance, the pressure gradient correction is expressed as

$$
\frac{\partial \bar{\Pi}^{\prime}}{\partial \chi}=\frac{\frac{\bar{m}_{i}}{\left(2 \pi \bar{\delta}_{i}\right)}-\sum_{j}\left(\bar{R}_{i}+\xi_{j} \bar{\delta}_{i}-\bar{\delta}_{i}\right) u_{x(i, j)}^{*} \Delta \xi}{\sum_{j}\left(\bar{R}_{i}+\xi_{j} \bar{\delta}_{i}-\bar{\delta}_{i}\right)\left(A_{\Pi}^{u_{x}} / A_{p}^{u_{x}}\right) \Delta \xi} .
$$

Step 9: The velocity corrections are given by Eqs. (A.17), (A.20), and (A.21). 


\section{Appendix B: Heat Trangfer In An Evaporating Liquid Layer}

Natural convection in an evaporating liquid film heated from below is modeled here to determine parameters affecting the magnitude of the Nusselt number in steady laminar convection. In the numerical heat pipe model developed in Chapter 3, the diffusion term in the energy equation is multiplied by a Nusselt number to account for natural convection in the rotating liquid film lining the wall of the evaporator section. Previous rotating heat pipe studies have shown that characterization of the Nusselt number in the evaporator section plays an important role in determining the overall thermal performance of the heat pipe (Marto 1984; Song et al. 2004; Takahashi et al. 1995b; Vasiliev and Khrolenok 1976).

Marto proposed using empirical correlations for natural convection in thin liquid films for determining the Nusselt number in the liquid film of a rotating heat pipe evaporator when natural convection is present (Marto 1984). The correlations of Marto and Gray $N u=0.14 R a^{1 / 3}$, Körner $N u=0.133 R a^{0.375}$, and Vasiliev and Khrolenok $N u=0.75 R a^{1 / 4}$ were suggested, where in each case the liquid film thickness, $\delta$, is used as a characteristic length for the Nusselt and Rayleigh numbers (Körner 1970; Marto and Gray 1971; Vasiliev and Khrolenok 1976). Although all three of these correlations have been suggested for heat pipes, they produce a wide variation in Nusselt number. For example, at a Rayleigh number of 6000, the correlation of Marto and Gray gives $N u=2.5$, the correlation of Körner gives $N u=3.5$, and the correlation of Vasiliev and Khrolenok produces $\mathrm{Nu}=6.6$.

After reviewing these references, one finds that the correlation by Marto and Gray is a standard formula for natural convection above a heated plate facing upward into an 
infinite medium [see (Cengel 1998) for example]. The correlation does follow the trend of their experimental data for water in a rotating cylindrical boiler, however, there is a large amount of scatter in the data and the reported experimental uncertainties are significant. The correlation of Vasiliev and Khrolenok is developed for a rotating heat pipe with a sharp step in the wall at the intersection of the adiabatic and evaporator sections. The applicability of this correlation to cylindrical and smoothly tapered heat pipes has been questioned in the past (Song et al. 2004). Finally, Körner's experimental study of heat transfer in thin films lining the wall of a rotating boiler was performed for natural convection in the turbulent regime $\left(10^{7}<R a<10^{10}\right)$. Because the liquid films developed in rotating heat pipes are very thin and there is a desire for operation with small temperature differences across the film, Rayleigh numbers developed in rotating heat pipes are typically considerably lower than this range.

Because of the wide range of results and the questions about the applicability of the above correlations to rotating heat pipes, heat transfer in an evaporating liquid film is modeled numerically, and an appropriate correlation is developed for low $\left(R a<10^{4}\right)$ Rayleigh numbers. The film is modeled in two-dimensional Cartesian coordinates. The predictions obtained from the planar geometry studied here are a first approximation to the axially rotating heat pipe application.

Predictions of the heat transfer coefficient in sealed rotating annuli with a heated outer wall and a cooled (rigid) inner wall indicate that, for a given Rayleigh number, the Nusselt numbers in the rotating annuli are lower than Nusselt numbers in (planar) Rayleigh-Bénard convection. Numerical and experimental studies indicate that the Coriolis force in the rotating system is responsible for inhibiting heat transfer in the 
rotating annulus. King and Wilson (King and Wilson 2005) indicate that the Coriolis force is closely related to the rotational Reynolds number, $\operatorname{Re}_{\phi}=\rho \Omega r_{m} d_{a} / \mu$, where $r_{m}$ is the mean radius of the annulus, and $d_{a}$ is the distance between the inner and outer rotating surfaces. Their numerical simulations of convection in rotating annuli showed that in the low rotational Reynolds number limit, Nusselt number predictions for the rotating annuli approach values for the planar Rayleigh-Bénard problem. Therefore, the results of the simulations of free-surface evaporation in Cartesian coordinates represent an upper bound on the heat transfer coefficient in the evaporator sections of cylindrical axially rotating heat pipes.

\section{Geometries Studied}

A layer of liquid shown in Fig. B.1 of length $L$ and height $\delta$ is studied here. The liquid is bounded by three solid walls. On the bottom, the plate is isothermal with temperature $T_{w}$ and the side surfaces are assumed adiabatic. Three different conditions for the top surface are modeled: (1) a rigid isothermal plate at $T_{s}$, (2) a free-surface without evaporation, or (3) a free-surface with evaporation. The fixed upper plate case will be used to compare the results from the numerical simulations to correlations for Rayleigh-Bénard convection. In the free-surface case, the vapor above the free-surface is modeled as isothermal saturated vapor at $T_{s}$.

\section{Mathematical Model and Numerical Analysis}

Governing Equations and Boundary Conditions

The governing equations are written for steady laminar two-dimensional flow and heat transfer. Furthermore, the fluid is modeled as Newtonian, Boussinesq- 


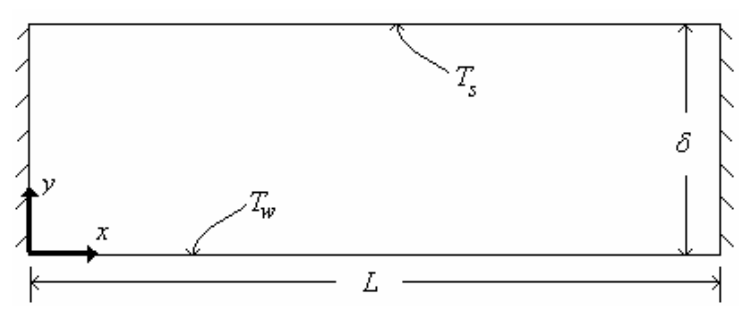

(a)

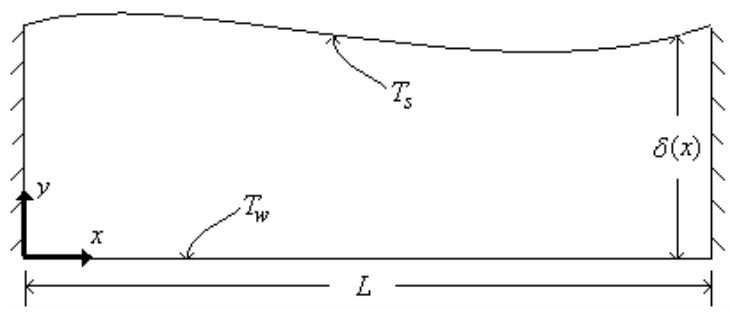

(b)

Figure B.1. Geometries studied. The fixed upper plate case (Rayleigh-Bénard) is shown in (a), while (b) is an illustration of the free-surface case.

incompressible, with constant viscosity, thermal conductivity, and specific heat. For the problem depicted in Fig. B.1, in Cartesian coordinates, the equations are

$$
\begin{aligned}
& \text { continuity: } \\
& \begin{array}{l}
\frac{\partial \mathrm{v}_{x}}{\partial x}+\frac{\partial \mathrm{v}_{y}}{\partial y}=0 \\
\text { momentum and energy: } \frac{\partial\left(\mathrm{v}_{x} \phi\right)}{\partial x}+\frac{\partial\left(\mathrm{v}_{y} \phi\right)}{\partial y}=\Gamma\left(\frac{\partial^{2} \phi}{\partial x^{2}}+\frac{\partial^{2} \phi}{\partial y^{2}}\right)+S^{\phi}+\nabla P^{\phi},
\end{array}
\end{aligned}
$$

where for

$$
\begin{aligned}
& \text { x-momentum: } \quad \phi=\mathrm{v}_{x}, S^{\phi}=0, \nabla P^{\phi}=-\frac{1}{\rho} \frac{\partial P}{\partial x} \text {, and } \Gamma=\frac{\mu}{\rho} \\
& \text { y-momentum: } \phi=\mathrm{v}_{y}, S^{\phi}=g \beta\left(T_{w}-T_{s}\right), \nabla P^{\phi}=-\frac{1}{\rho} \frac{\partial P}{\partial y} \text {, and } \Gamma=\frac{\mu}{\rho} \\
& \text { energy: } \quad \phi=T, S^{\phi}=0, \nabla P^{\phi}=0 \text {, and } \Gamma=\frac{k}{\rho C_{p}} .
\end{aligned}
$$

For a fixed top plate (the Rayleigh-Bénard problem) velocities $\left(\mathrm{v}_{x}\right.$ and $\left.\mathrm{v}_{y}\right)$ are set to zero on all four boundaries, the temperature of the top and bottom plates are specified as, $T(x, y=\delta)=T_{s} \quad$ and $\quad T(x, y=0)=T_{w}, \quad$ and the side-walls are insulated, $\partial T /\left.\partial x\right|_{x=0}=\partial T /\left.\partial x\right|_{x=L}=0$. For a free-surface, the boundary conditions for the bottom and side boundaries are the same as those listed above, however, the boundary conditions at the liquid-vapor interface $(y=\delta$ ) are written as 


$$
T=T_{s}
$$

with evaporation: $\rho h_{f g}(\mathbf{v} \cdot \mathbf{n})=-k(\nabla T \cdot \mathbf{n})$, no evaporation: $\mathbf{v} \cdot \mathbf{n}=0$

$$
\begin{gathered}
-2 H \sigma\left(n_{x}^{2}+n_{y}^{2}\right)=\rho\left(1-\rho / \rho_{v}\right)(\mathbf{v} \bullet \mathbf{n})^{2}\left(n_{x}^{2}+n_{y}^{2}\right)+\tau_{x x} n_{x}^{2}+2 \tau_{x y} n_{x} n_{y}+\tau_{y y} n_{y}^{2}+P \\
\tau_{x x} n_{x} n_{y}+\tau_{x y}\left(n_{y}^{2}-n_{x}^{2}\right)-\tau_{y y} n_{y} n_{x}=0,
\end{gathered}
$$

where Eq. (B.6) is a temperature specification at the interface, Eq. (B.7) is the kinematic condition at the interface (derived from an energy balance at the interface for the evaporation case), Eq. (B.8) is the normal stress jump condition, and Eq. (B.9) is the tangential stress balance. The unit normal vector is given by $\mathbf{n}=n_{x} \mathbf{e}_{x}+n_{y} \mathbf{e}_{y}=\left(-\delta^{\prime} \mathbf{e}_{x}+\mathbf{e}_{y}\right) / \sqrt{1+\delta^{\prime 2}}$, with $\delta^{\prime}=\mathrm{d} \delta / \mathrm{d} x$, the mean curvature of the interface is $H=\frac{1}{2} \delta^{\prime \prime}\left[1+\delta^{\prime 2}\right]^{-3 / 2}$ with $\delta^{\prime \prime}=\mathrm{d}^{2} \delta / \mathrm{d} x^{2}$, and the stress components are expressed as $\tau_{x x}=-2 \mu\left(\partial v_{x} / \partial x\right), \tau_{y y}=-2 \mu\left(\partial \mathrm{v}_{y} / \partial y\right)$, and $\tau_{x y}=-\mu\left(\partial \mathrm{v}_{x} / \partial y+\partial \mathrm{v}_{y} / \partial x\right)$. The boundary conditions at the free-surface are written after assuming that the vapor above the interface is stress free with $P_{v}=0$.

\section{Coordinate Transformations and Dimensionless Parameters}

The problem is mapped into a rectangular computational space by defining the independent variables as

$$
\eta=y /(\bar{\delta} L) \text { and } z=x / L \text {, where } \bar{\delta}=\delta / L \text { and } \bar{\delta}=\bar{\delta}(z) .
$$

With this transformation, the bottom and top boundaries are located at $\eta=0$ and $\eta=1$, respectively, regardless of the liquid layer thickness $\bar{\delta}$. The transformation does, however, introduce $\bar{\delta}$ and its derivatives with respect to $\mathrm{z}$ into the governing equations and boundary conditions. 
Velocity, temperature, volume expansion coefficient, and acceleration are made dimensionless by

$$
\mathbf{u}=\mathbf{v} / \mathrm{v}_{c h}, \Lambda=\left(T-T_{s}\right) /\left(T_{w}-T_{s}\right), \bar{\beta}=\beta\left(T_{w}-T_{s}\right) \text {, and } \bar{g}=g L / \mathrm{v}_{c h}^{2} \text {, }
$$

where the characteristic velocity is $\mathrm{v}_{c h}=\mu /(\rho L)$. Using these expressions, the governing equations become

continuity: $\quad \frac{\partial u_{x}}{\partial z}+\frac{1}{\bar{\delta}} \frac{\partial u_{y}}{\partial \eta}-\frac{\bar{\delta}^{\prime} \eta}{\bar{\delta}} \frac{\partial u_{x}}{\partial \eta}=0$

momentum and energy: $\frac{\partial}{\partial z}\left(u_{x} \bar{\phi}-\bar{\Gamma} \frac{\partial \bar{\phi}}{\partial z}\right)+\frac{1}{\bar{\delta}} \frac{\partial}{\partial \eta}\left(u_{y} \bar{\phi}-\frac{\bar{\Gamma}}{\bar{\delta}} \frac{\partial \bar{\phi}}{\partial \eta}\right)-\frac{\bar{\delta}^{\prime} \eta}{\bar{\delta}} \frac{\partial\left(u_{x} \bar{\phi}\right)}{\partial \eta}$

$$
=\bar{S}^{\bar{\phi}}+\nabla \Pi^{\bar{\phi}}+C^{\bar{\phi}}
$$

where for

$$
\begin{aligned}
& \text { x-momentum: } \bar{\phi}=u_{x}, \bar{S}^{\bar{\phi}}=0, \nabla \Pi^{\bar{\phi}}=-\frac{\partial \Pi}{\partial x}-\frac{\bar{\delta}^{\prime} \eta}{\bar{\delta}} \frac{\partial \Pi}{\partial \eta} \text {, and } \bar{\Gamma}=\frac{1}{\operatorname{Re}} \\
& \text { y-momentum: } \bar{\phi}=u_{y}, \bar{S}^{\bar{\phi}}=\bar{g} \bar{\beta} \theta, \nabla \Pi^{\bar{\phi}}=-\frac{1}{\bar{\delta}} \frac{\partial \Pi}{\partial \eta} \text {, and } \bar{\Gamma}=\frac{1}{\operatorname{Re}} \\
& \text { energy: } \bar{\phi}=\Lambda, \bar{S}^{\bar{\phi}}=0, \nabla \Pi^{\bar{\phi}}=0 \text {, and } \bar{\Gamma}=\frac{1}{P e} \text {. }
\end{aligned}
$$

The following dimensionless parameters appear in Eqs. (B.12)-(B.16): the dimensionless modified pressure $\Pi=P /\left(\rho \mathrm{v}_{c h}^{2}\right)$, the Reynolds number $\operatorname{Re}=\rho \mathrm{v}_{c h} L / \mu$, and the Péclet number $P e=\rho C_{p} \mathrm{v}_{c h} L / k$. The coordinate transformation used in Eq. (B.10) results in pseudo-diffusion terms (terms stemming from the diffusion terms which multiply derivatives of $\bar{\delta}$ with respect to $z$ ) appearing as source terms in the momentum and energy equations. The pseudo-diffusion terms are given by 


$$
C^{\bar{\phi}}=\bar{\Gamma}\left(\frac{2 \eta \bar{\delta}^{\prime 2}}{\bar{\delta}^{2}} \frac{\partial \bar{\phi}}{\partial \eta}-\frac{2 \eta \bar{\delta}^{\prime}}{\bar{\delta}} \frac{\partial^{2} \bar{\phi}}{\partial \eta \partial z}-\frac{\eta \bar{\delta}^{\prime \prime}}{\bar{\delta}} \frac{\partial \bar{\phi}}{\partial \eta}+\frac{\eta^{2} \bar{\delta}^{\prime 2}}{\bar{\delta}^{2}} \frac{\partial^{2} \bar{\phi}}{\partial \eta^{2}}\right)
$$

where $\bar{\delta}^{\prime \prime}=\mathrm{d}^{2} \bar{\delta} / \mathrm{d} z^{2}$.

To simplify integration of the governing equations, $x$ and $\eta$-direction velocities $\left(u_{x}\right.$ and $u_{\eta}$, respectively) are retained, and the momentum equations are expressed in the $x$ and $\eta$-directions (Faghri et al. 1984). The coordinate transformation in Eq. (B.10) shows that

$$
u_{y}=u_{\eta}+\bar{\delta}^{\prime} \eta u_{x}
$$

and $\quad[\eta-$ momentum $]=[y-$ momentum $]-\bar{\delta}^{\prime} \eta[x-$ momentum $]$.

Finally, with Eqs. (B.18) and (B.19) the governing equations become

continuity: $\quad \frac{1}{\bar{\delta}} \frac{\partial}{\partial z}\left(\bar{\delta} u_{x}\right)+\frac{1}{\bar{\delta}} \frac{\partial u_{\eta}}{\partial \eta}=0$

momentum and energy: $\frac{1}{\bar{\delta}} \frac{\partial}{\partial z}\left(\bar{\delta} u_{x} \bar{\phi}-\bar{\Gamma} \bar{\delta} \frac{\partial \bar{\phi}}{\partial z}\right)+\frac{1}{\bar{\delta}} \frac{\partial}{\partial \eta}\left(u_{\eta} \bar{\phi}-\frac{\bar{\Gamma}}{\bar{\delta}} \frac{\partial \bar{\phi}}{\partial \eta}\right)$

$$
=\bar{S}^{\bar{\phi}}+\nabla \Pi^{\bar{\phi}}+C_{1}^{\bar{\phi}}+C_{2}^{\bar{\phi}},
$$

where for

$$
\begin{aligned}
& \text { x-momentum: } \bar{\phi}=u_{x}, \bar{S}^{\bar{\phi}}=0, \nabla \Pi^{\bar{\phi}}=-\frac{\partial \Pi}{\partial x}-\frac{\overline{\delta^{\prime}} \eta}{\bar{\delta}} \frac{\partial \Pi}{\partial \eta} \text {, and } \bar{\Gamma}=\frac{1}{\mathrm{Re}} \\
& \eta \text {-momentum: } \bar{\phi}=u_{\eta}, \bar{S}^{\bar{\phi}}=\bar{g} \bar{\beta} \Lambda, \nabla \Pi^{\bar{\phi}}=-\frac{\left(1+\eta^{2}{\overline{\delta^{\prime}}}^{2}\right)}{\bar{\delta}} \frac{\partial \Pi}{\partial \eta}+\eta \bar{\delta}^{\prime} \frac{\partial \Pi}{\partial z}, \\
& \text { and } \bar{\Gamma}=\frac{1}{\mathrm{Re}} \\
& \text { energy: } \quad \bar{\phi}=\Lambda, \bar{S}^{\bar{\phi}}=0, \nabla \Pi^{\bar{\phi}}=0 \text {, and } \bar{\Gamma}=\frac{1}{P e} .
\end{aligned}
$$


The pseudo-diffusion terms are given by

$$
C_{1}^{\bar{\phi}}=\bar{\Gamma}\left(\frac{2 \eta \bar{\delta}^{\prime 2}}{\bar{\delta}^{2}} \frac{\partial \bar{\phi}}{\partial \eta}-\frac{2 \eta \bar{\delta}^{\prime}}{\bar{\delta}} \frac{\partial^{2} \bar{\phi}}{\partial \eta \partial z}-\frac{\eta \bar{\delta}^{\prime \prime}}{\bar{\delta}} \frac{\partial \bar{\phi}}{\partial \eta}+\frac{\eta^{2}{\overline{\delta^{\prime}}}^{2}}{\bar{\delta}^{2}} \frac{\partial^{2} \bar{\phi}}{\partial \eta^{2}}+\frac{\overline{\delta^{\prime}}}{\bar{\delta}} \frac{\partial \bar{\phi}}{\partial z}\right)
$$

and for the x-momentum and energy equations $C_{2}^{u_{x}}=C_{2}^{\theta}=0$. For the $\eta$-momentum equation

$$
C_{2}^{u_{\eta}}=\frac{1}{\operatorname{Re}}\left(\frac{2 \bar{\delta}^{\prime}}{\bar{\delta}^{2}} \frac{\partial u_{\eta}}{\partial \eta}+2 \eta \overline{\delta^{\prime \prime}} \frac{\partial u_{x}}{\partial z}+\eta \bar{\delta}^{\prime \prime \prime} u_{x}-\frac{\eta \bar{\delta}^{\prime} \bar{\delta}^{\prime \prime}}{\bar{\delta}} u_{x}+\frac{2 \eta \bar{\delta}^{\prime 2} \bar{\delta}^{\prime \prime}}{\bar{\delta}^{2}} u_{x}\right)
$$

where $\bar{\delta}^{\prime \prime \prime}=\mathrm{d}^{3} \bar{\delta} / \mathrm{d} z^{3}$.

The dimensionless boundary conditions, for the Rayleigh-Bénard problem are: $u_{x}=u_{\eta}=0$ on all four surfaces, and for the energy equation $\Lambda(z, \eta=0)=1$, $\Lambda(z, \eta=1)=0, \partial \Lambda /\left.\partial z\right|_{z=0}=\partial \Lambda /\left.\partial z\right|_{z=1}=0$. For the free-surface case, the bottom and side conditions are the same as those listed above. The free-surface $(\eta=1)$ conditions become

$$
\Lambda=0
$$

with evaporation: $u_{\eta}=-\frac{J a}{\operatorname{Pr}}\left(\frac{\left(1+\bar{\delta}^{2}\right)}{\bar{\delta}} \frac{\partial \Lambda}{\partial \eta}-\bar{\delta}^{\prime} \frac{\partial \Lambda}{\partial z}\right)$, no evaporation: $u_{\eta}=0$

$$
\begin{gathered}
\frac{\bar{\delta}^{\prime \prime}}{C a}\left(1+{\overline{\delta^{\prime}}}^{2}\right)^{-3 / 2}=\left(1-\rho / \rho_{v}\right) u_{\eta}^{2}+\frac{2 \bar{\delta}^{\prime}}{\operatorname{Re}\left(1+\bar{\delta}^{\prime 2}\right)}\left(\frac{\partial u_{\eta}}{\partial z}+\bar{\delta}^{\prime \prime} u_{x}\right)-\frac{2}{\bar{\delta} \operatorname{Re}} \frac{\partial u_{\eta}}{\partial \eta}+\Pi \\
\frac{\partial u_{x}}{\partial \eta}=\frac{\overline{\delta \delta^{\prime}}}{\left(1+\delta^{\prime 2}\right)} \frac{\partial u_{x}}{\partial z}-\frac{\delta^{\prime}}{\left(1+\delta^{\prime 2}\right)} \frac{\partial u_{\eta}}{\partial \eta}-\frac{\bar{\delta}\left(1-\bar{\delta}^{\prime 2}\right)}{\left(1+\bar{\delta}^{\prime 2}\right)^{2}}\left(\frac{\partial u_{\eta}}{\partial z}+\bar{\delta}^{\prime \prime} u_{x}\right)
\end{gathered}
$$

with the Capillary, Jakob, and Prandtl numbers defined, respectively, as $C a=\rho \mathrm{v}_{c h}^{2} L / \sigma$, $J a=C_{p}\left(T_{w}-T_{s}\right) / h_{f g}$, and $\operatorname{Pr}=\mu C_{p} / k$ 


\section{Numerical Solution}

Equations (B.20)-(B.24) subject to the dimensionless boundary conditions are solved numerically. The equations are discretized on a staggered grid using a control volume method. Throughout the simulations, uniform grids in both the $z$ and $\eta$-directions are used. False boundary nodes located at distances $\Delta z / 2$ and $\Delta \eta / 2$ beyond the horizontal and vertical boundaries of the solution domain are used to specify the values of the boundary conditions. Throughout the discretization, pressure and temperature are stored at the main grid nodes, while velocities are stored at the faces of the control volumes surrounding each main grid node. In the momentum and energy equations, the pseudodiffusion terms are treated as source terms, second-order centered-differences are used to approximate the diffusion terms, and the QUICK method (Leonard 1979) is used for the convective terms. Derivatives appearing in the pseudo-diffusion terms and the boundary conditions are discretized using second-order finite-differences. The discrete equations are solved using the SIMPLER method (Patankar 1980). For cases where the upper boundary is free to move, the temperature specification [Eq. (B.27)] is applied as a boundary condition to the energy equation, the kinematic condition [Eq. (B.28)] is applied to the $\eta$-momentum equation, the discrete form of the shear stress balance [Eq. (B.30)] is applied to the $x$-momentum equation, and the normal stress jump condition [Eq. (B.29)] is used to determine the liquid layer thickness.

The layer thickness is determined by iteration. A constant layer thickness is assumed and governing equations are solved without regard for the normal stress condition. If the normal stress condition is not satisfied by the solution, Eq. (B.29) is used to update the layer thickness and the process is repeated until the normal stress condition is satisfied. 
The second-order ordinary differential equation in Eq. (B.29) is solved at each iteration using a fourth-order Runge-Kutta method with shooting to handle the boundary conditions $\bar{\delta}(z=0)=\bar{\delta}(z=1)=\bar{\delta}_{0}$, where $\bar{\delta}_{0}$ is the initial guess for the (uniform) layer thickness. When the governing equations are solved by the SIMPLER method, the pressure is solved for to within an additive constant (only pressure gradients are required for a solution). When updating the interface location, this additive constant appearing in the pressure term of Eq. (B.29) is determined so that the volume of the fluid in the solution domain after the surface update is equal to the initial guessed volume.

Convergence Criteria, Observed Convergence Rates, And Error ESTIMATES

In the SIMPLER method, iterative convergence of the continuity, momentum, and energy equations is assumed after the residuals of each equation have been reduced three orders of magnitude from the residuals calculated with the initial guessed solution. The conduction solution (zero velocity and linear temperature profile) is used as an initial guess. Similarly, in free-surface problems, the layer thickness is updated until the residual of the normal stress condition is reduced three orders of magnitude below the residual calculated for the initial (assumed flat) surface profile.

Grid convergence rates and error estimates have been calculated for the code simulating both the Rayleigh-Bénard problem and the free-surface evaporation problem for water in a 4 x 1 [(length) x (height)] box. Throughout the grid convergence studies, the layer thickness is specified as $0.5 \mathrm{~mm}$, the temperature difference across the layer is assumed to be $12 \mathrm{~K}$, and the layer is experiencing acceleration at $45 \mathrm{~g}\left(441 \mathrm{~m} / \mathrm{s}^{2}\right)$. For 
saturated liquid water at $373 \mathrm{~K}$, these parameters correspond to a Rayleigh number of $R a=10^{4}$.

Simulations of Rayleigh-Bénard and free-surface convection with evaporation were performed on grids with $[z] \times[\eta]$ resolutions of $96 \times 24,128 \times 32$, and $160 \times 40$. An additional grid of 200 x 50 was also used for the free-surface evaporation case. The observed order of the method was then calculated along with error bounds. Error bounds were calculated with the Grid Convergence Index (GCI) (Roache 1998), using the average Nusselt number along the bottom plate as the parameter of interest. In terms of dimensionless variables, the average Nusselt number is calculated from

$$
N u=-\int_{0}^{1}\left(\partial \Lambda /\left.\partial \eta\right|_{\eta=0}\right) \mathrm{d} z
$$

Results of the grid convergence study are shown in Table B.1. The results indicate that for the Rayleigh-Bénard problem the calculated apparent order of the method is second order. Since a second-order discretization has been used throughout the code development, the expected apparent order should be near two. For the finest grid used (160 x 40) less than one percent error can be expected for the rigid upper plate case. For the free-surface problem the apparent order is smaller than two. Chen and Floryan (Chen and Floryan 1994) also observed an apparent order less than the formal discretization order when solving free-surface problems using analytical mapping techniques. The 200 x 50 grid was needed to reduce the errors to an acceptable level (3.4\%).

VALIDATION

The capability of the code to accurately simulate natural convection was assessed by comparing the results produced by the code to a benchmark numerical solution for natural convection in an air-filled, square, side-heated cavity (de Vahl Davis 1983). In 
Table B.1. Grid convergence studies for (a) the Rayleigh-Bénard problem and (b) the free-surface problem with evaporation. Both are carried out in a $4 \times 1$ box.

\begin{tabular}{|c|c|c|c|c|c|c|c|c|}
\hline \multicolumn{4}{|c|}{$\begin{array}{l}\text { (a) Rayleigh-Benard Convection } \\
\text { in } 4 \times 1 \text { box for } R a=10^{4}\end{array}$} & \multicolumn{5}{|c|}{$\begin{array}{l}\text { (b) Free Surface Convection with } \\
\text { Evaporation in } 4 \times 1 \text { box for } \mathrm{Ra}=10^{4}\end{array}$} \\
\hline & \multicolumn{3}{|c|}{ grid size } & & \multicolumn{3}{|c|}{ grid size } & \\
\hline & $96 \times 24$ & $128 \times 32$ & $160 \times 40$ & & $96 \times 24$ & $128 \times 32$ & $160 \times 40$ & $200 \times 50$ \\
\hline $\mathrm{Nu}$ & 2.490 & 2.506 & 2.513 & $\mathrm{Nu}$ & 2.937 & 3.004 & 3.041 & 3.069 \\
\hline \multicolumn{4}{|c|}{$\begin{array}{l}(\mathbf{9 6} \times 24)->(\mathbf{1 6 0} \times 40) \text { apparent } \text { order }=\mathbf{2 . 2} \\
(\mathbf{1 6 0} \times \mathbf{4 0}) \mathbf{G C I}=\mathbf{0 . 5 3} \% \\
(128 \times 32) \mathrm{GCI}=1.2 \%\end{array}$} & \multicolumn{5}{|c|}{$\begin{array}{l}(96 \times 24)->(160 \times 40) \text { apparent order }=1.3 \\
(\mathbf{1 2 8 \times 3 2})->(\mathbf{2 0 0} \times \mathbf{5 0}) \text { apparent order }=\mathbf{1 . 3} \\
(\mathbf{2 0 0} \times \mathbf{5 0}) \mathbf{G C I}=\mathbf{3 . 4 \%} \\
(160 \times 40) \text { GCI }=4.5 \% \\
(128 \times 32) \text { GCI }=6.0 \%\end{array}$} \\
\hline
\end{tabular}

bold values indicate the grid selected for calculations

this benchmark problem, the Rayleigh number is based on the length (or height) of the square cavity, and the temperature difference between hot and cold side-walls. The horizontal walls are assumed insulated. The code described above was used to solve this problem on a $101 \times 101$ grid for Rayleigh numbers of $10^{3}, 10^{4}$, and $10^{5}$. Results of the comparison between the present solution and the benchmark are shown in Table B.2. In order from top to bottom, the table displays: the average Nusselt number on the hot plate, the maximum Nusselt number on the hot plate followed by the $\eta$-location where that maximum occurs, the minimum Nusselt number on the hot plate followed by location, maximum horizontal velocity at the vertical mid-plane $(z=1 / 2)$ with $\eta$-location, and maximum vertical velocity at the horizontal mid-plane $(\eta=1 / 2)$ with $z$-location. For a direct comparison with the benchmark, velocities in the table are presented after a multiplication by the Prandtl number. The thermal diffusivity is used to normalize the velocity in the benchmark solution of de Vahl Davis (de Vahl Davis 1983), whereas here the momentum diffusivity is used [see Eq. (B.11)]. The results in the table show agreement within $2 \%$ between the benchmark and the present solution for the cases and variables considered. 
Table B.2. Comparison between a benchmark solution for natural convection in a square side-heated cavity and the present numerical solution.

\begin{tabular}{|c|c|c|c|c|c|c|c|c|c|}
\hline & \multicolumn{9}{|c|}{$\mathrm{Ra}$} \\
\hline & \multicolumn{3}{|c|}{$10^{3}$} & \multicolumn{3}{|c|}{$10^{4}$} & \multicolumn{3}{|c|}{$10^{5}$} \\
\hline & Benchmark & Present & \% error & Benchmark & Present & \% error & Benchmark & Present & \% error \\
\hline $\mathrm{Nu}$ & 1.117 & 1.116 & 0.05 & 2.238 & 2.253 & 0.68 & 4.509 & 4.529 & 0.45 \\
\hline $\mathrm{Nu}_{\max }$ & 1.505 & 1.517 & 0.81 & 3.528 & 3.552 & 0.68 & 7.717 & 7.765 & 0.617 \\
\hline$\eta$-location & 0.092 & 0.066 & & 0.143 & 0.136 & & 0.081 & 0.066 & \\
\hline $\mathrm{Nu}_{\min }$ & 0.692 & 0.678 & 2.03 & 0.586 & 0.588 & 0.29 & 0.729 & 0.739 & 1.34 \\
\hline$\eta$-location & 1 & 1 & & 1 & 1 & & 1 & 1 & \\
\hline$(\mathrm{Pr})^{*} \mathrm{u}_{\mathrm{x}, \max }$ & 3.649 & 3.701 & 1.43 & 16.178 & 16.15 & 0.17 & 34.73 & 34.17 & 1.62 \\
\hline$\eta$-location & 0.813 & 0.813 & & 0.823 & 0.823 & & 0.855 & 0.854 & \\
\hline$(\operatorname{Pr})^{*} \mathrm{u}_{\eta, \max }$ & 3.697 & 3.744 & 1.27 & 19.617 & 19.75 & 0.67 & 68.59 & 68.92 & 0.49 \\
\hline z-location & 0.178 & 0.177 & & 0.119 & 0.116 & & 0.066 & 0.066 & \\
\hline
\end{tabular}

Simulations of a square side-heated cavity with free-surface (without evaporation) boundary conditions at the top surface were also compared to a reference case from the open literature (Cuvelier and Driessen 1986). In the reference calculation, sliding contact points are assumed at the side-walls with $\bar{\delta}^{\prime}(z=0)=\bar{\delta}^{\prime}(z=1)=0$, rather than the fixed contact points used here. The sliding contact point boundary conditions were introduced into the code to allow for a direct comparison with the benchmark. In particular, the calculated layer thickness at the side-walls is compared to the predictions from the benchmark solution. With a Grashof number ( $G r=R a / P r$ ) of 20,000 and an Ohnesorge number $(O h=\mu / \sqrt{\rho \sigma L}$ ) of 0.01 , the layer thickness predicted by the code (with a $51 \mathrm{x}$ 51 grid) are $\bar{\delta}(z=0)=1.007$ and $\bar{\delta}(z=1)=0.993$. The benchmark predictions (Cuvelier and Driessen 1986) are $\bar{\delta}(z=0)=1.009$ and $\bar{\delta}(z=1)=0.992$. These comparisons suggest that the code is predicting the free-surface location properly.

\section{RESULTS}

\section{Streamlines, Isotherms, and Surface Deformation}

Streamlines and isotherms for the fixed upper plate and free-surface evaporation cases are shown in Fig. B.2 for $R a=10^{4}$. The streamline plots show the development of 
roll cells in both the fixed upper plate and free-surface evaporation cases. From the temperature contour plots, notice that for the free-surface evaporation case the hot and cold fluids penetrate farther into the cavity than for the Rayleigh-Bénard case, indicating enhanced mixing and higher heat transfer coefficients. The shape of the free-surface for the evaporation case is shown in Fig. B.3. The scale in the figure shows that the liquidvapor interface is essentially flat under the conditions simulated. This small surface

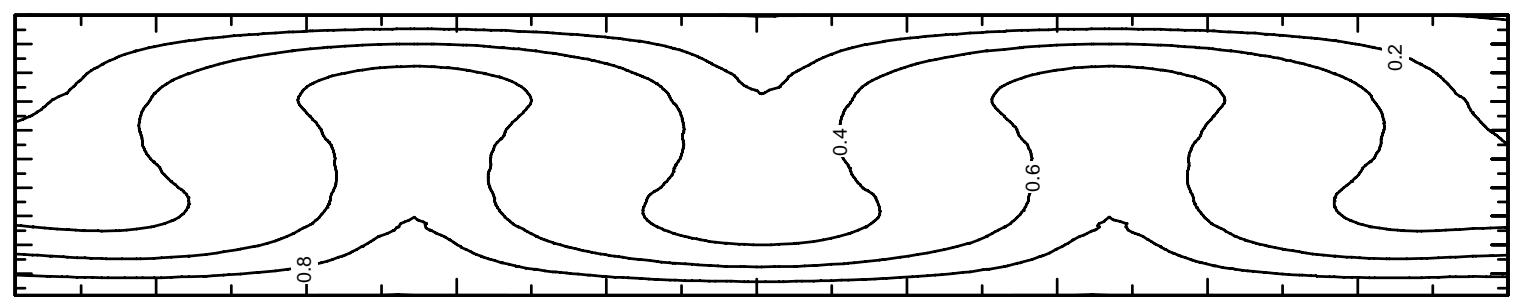

(a)

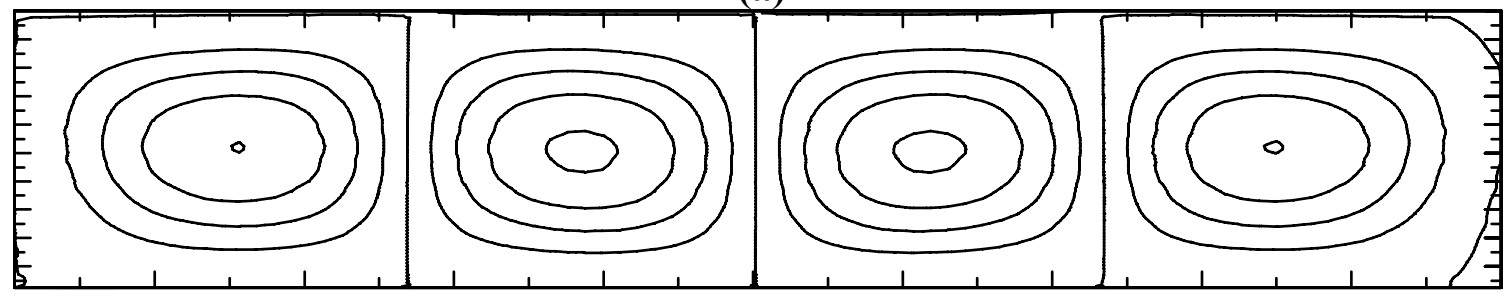

(b)

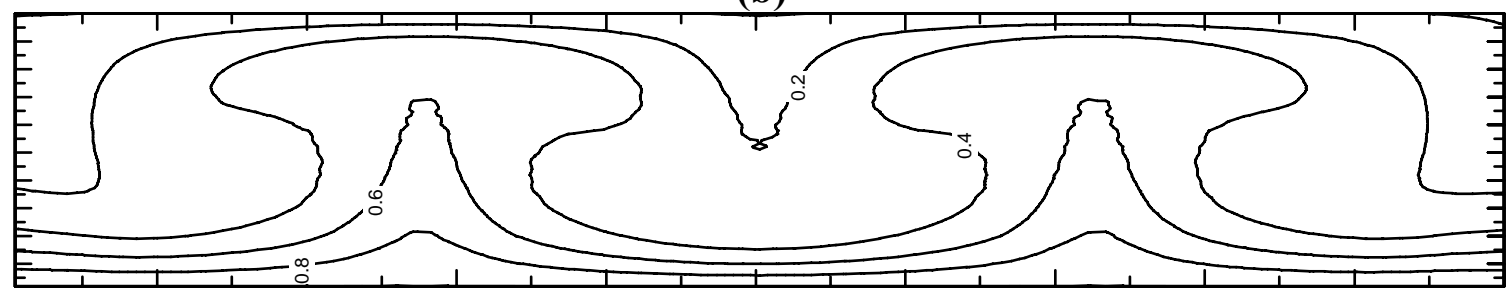

(c)
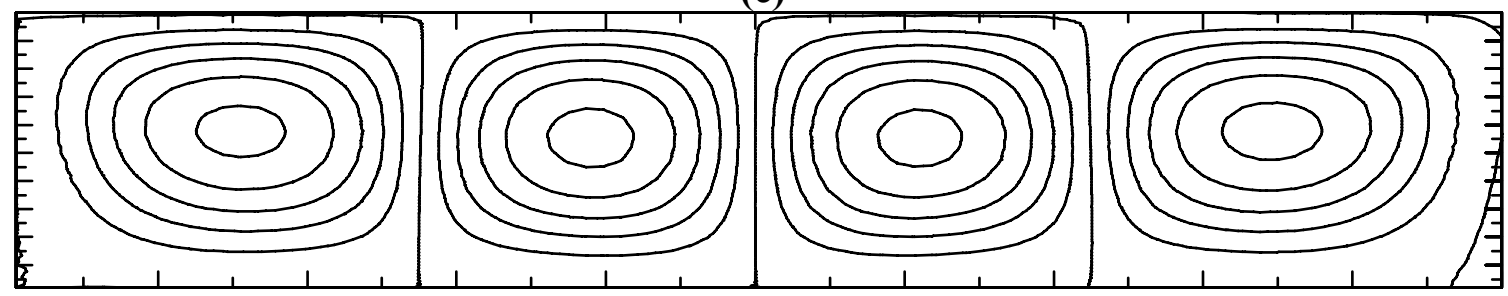

(d)

Figure B.2. Streamlines and isotherms for natural convection in a $4 \times 1$ system for the fixed upper plate case [isotherms in (a) and streamlines in (b)] and for the case with free-surface evaporation [isotherms in (c) and streamlines in (d)]. Both cases simulate convection in water with $R a=10^{4}$. 
deflection is also expected in the rotating heat pipe application. Cuvelier and Driessen (Cuvelier and Driessen 1986) show that the surface deflection is determined by the ratio of buoyancy forces to surface tension forces (the Bond number). High buoyancy forces (high accelerations) tend to flatten the interface.

\section{Nusselt Numbers}

Simulations were run with a $4 \times 1$ aspect ratio enclosure to determine the Nusselt number using saturated water and methanol working fluids for the fixed upper plate, freesurface, and free-surface with evaporation cases. In each case, the heat transfer is simulated for the Rayleigh number ranging from $10^{3}$ to $10^{4}$. Results of the simulations are shown in Fig. B.4. A liquid layer with a thickness of $0.5 \mathrm{~mm}$ and a temperature difference of $12 \mathrm{~K}$ is modeled, which are typical of the values encountered in heat pipe applications. For water the acceleration ranges from $4.5 \mathrm{~g}$ to $45 \mathrm{~g}$ and for methanol the acceleration ranges from $3.8 \mathrm{~g}$ to $38 \mathrm{~g}$.

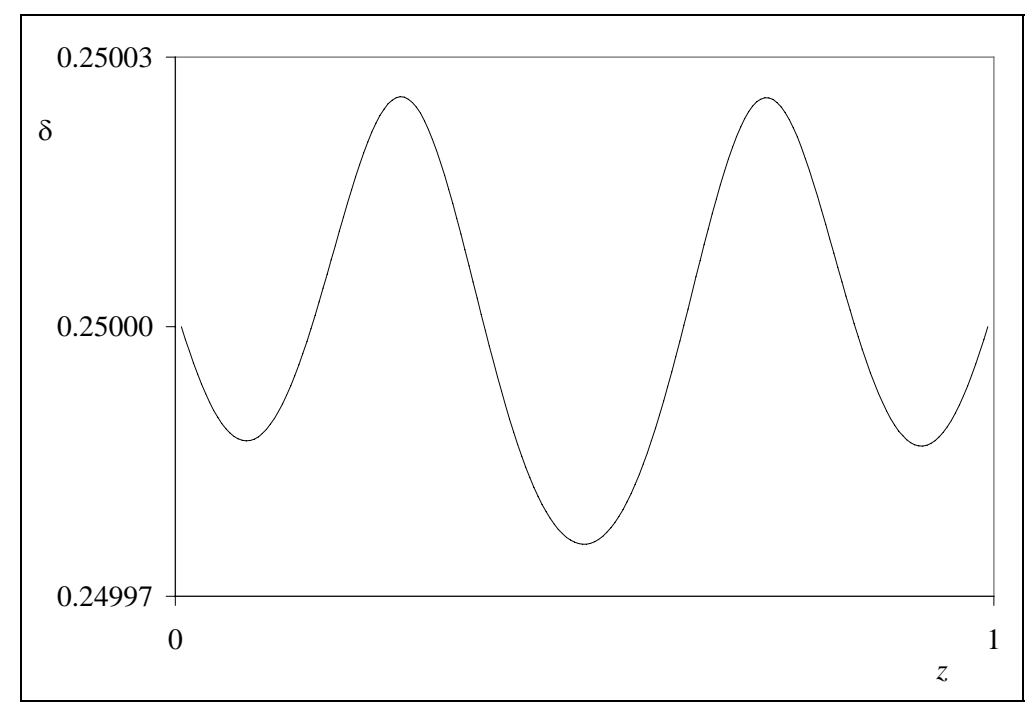

Figure B.3. Surface profile for the free-surface evaporation case. Results are for convection in water with $R a=10^{4}$. 


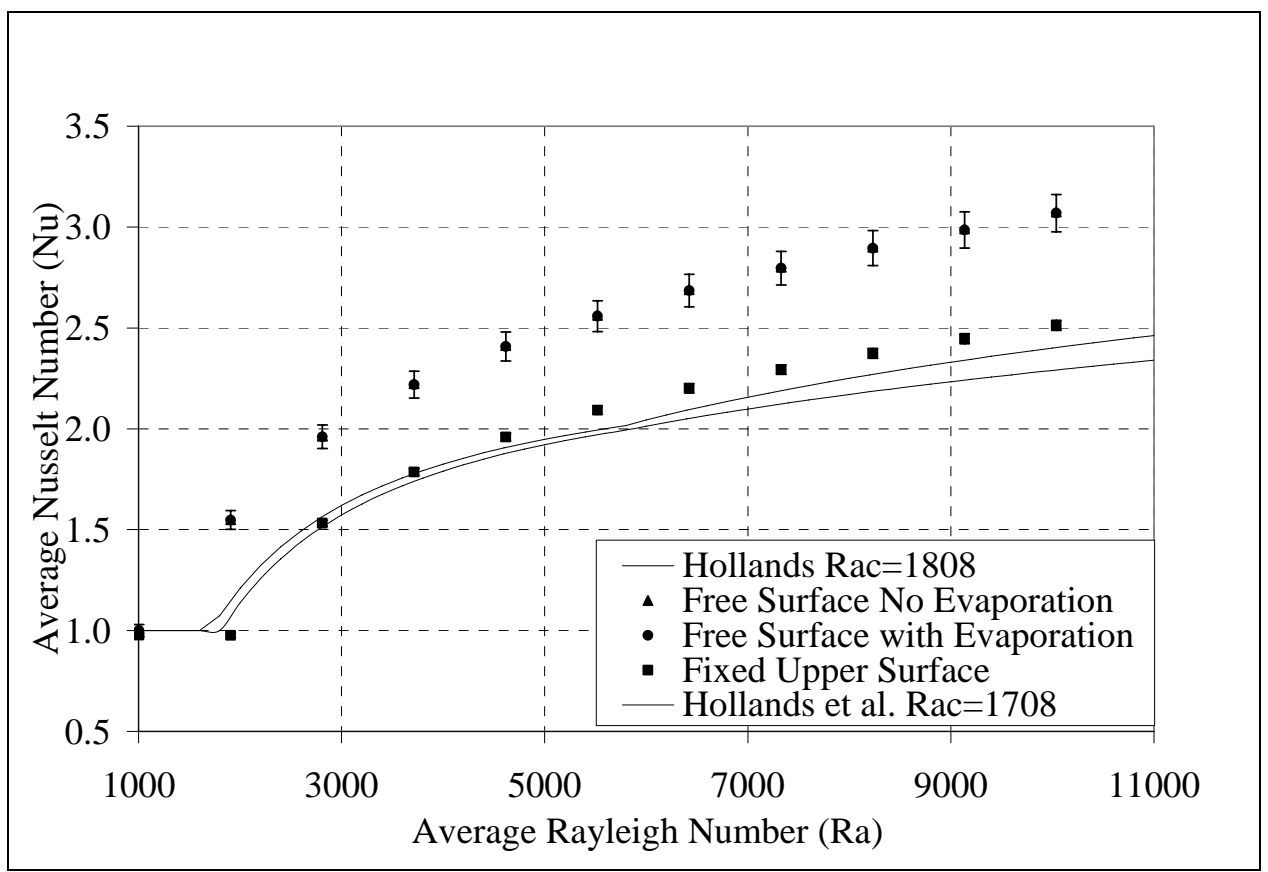

(a)

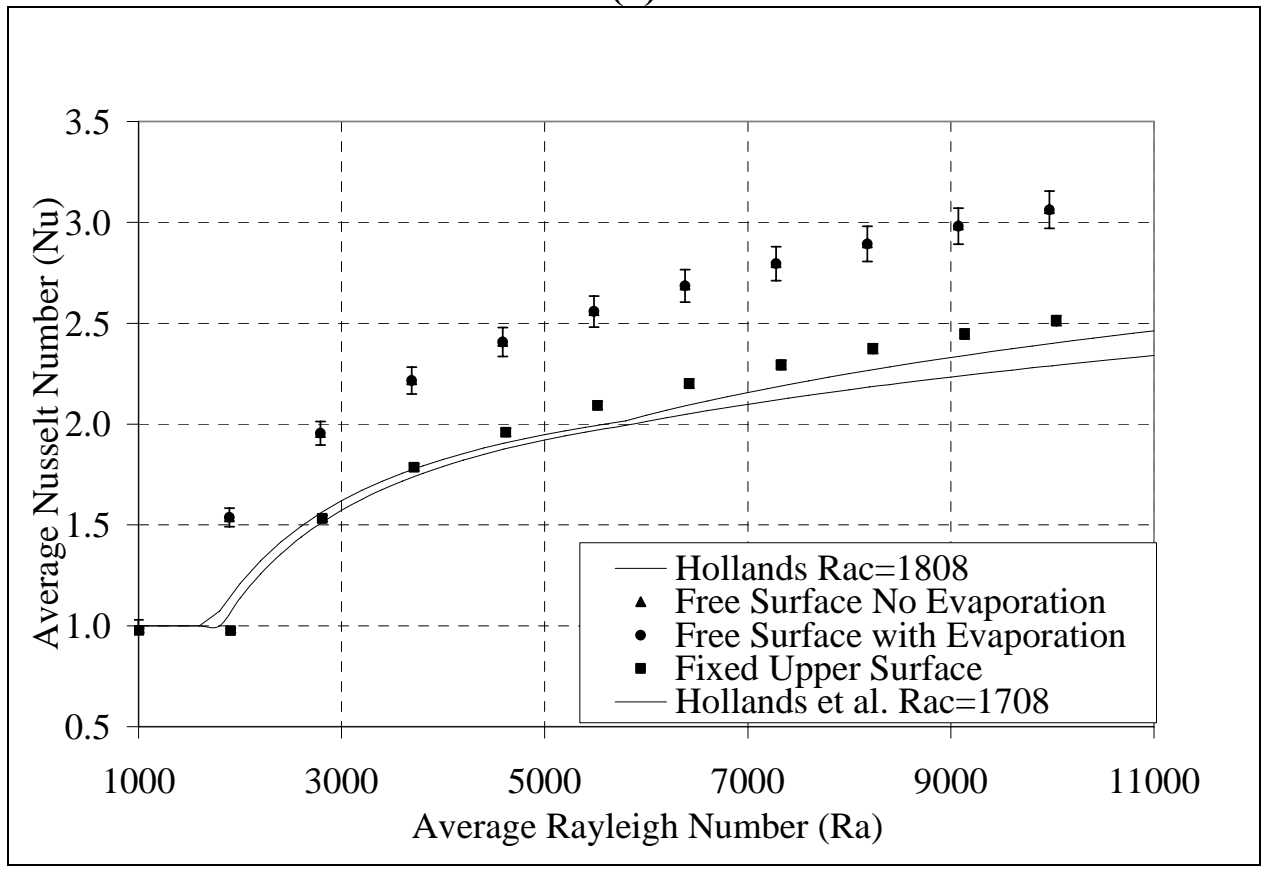

(b)

Figure B.4. Comparing convection in a $4 \times 1$ box for the fixed upper surface, freesurface, and free-surface with evaporation cases. In (a) the working fluid is water, and in (b) the fluid is methanol.

For the fixed upper plate case, agreement is observed between the present calculations and the correlations of Hollands et al. (Hollands et al. 1975) and Hollands (Hollands 1983). The correlation of Hollands et al. (Hollands et al. 1975) is developed 
for convection in a fluid layer of infinite aspect ratio, whereas the correlation of Hollands (Hollands 1983) is for the $4 \times 1$ enclosure modeled here with a critical Rayleigh number of $R a_{c}=1808$ taken from Catton (Catton 1972). The critical Rayleigh number is the value at which the transition from pure conduction $(N u=1)$ to convection $(N u>1)$ occurs. The agreement between the correlating equations shows that the sidewalls of the $4 \times 1$ enclosure only slightly affect the heat transfer compared to the infinite aspect ratio case.

The Nusselt numbers predicted for the free-surface cases are higher than those for the fixed upper plate case, with a critical Rayleigh number (determined with some numerical experiments) near 1600. This critical value is slightly larger than the value of 1101 predicted from a stability analysis with free-surface conditions for the infinite aspect ratio case (Chandrasekhar 1961). Furthermore, the surface evaporation has a negligible influence on the Nusselt number compared to the free-surface case without evaporation.

The surface evaporation will affect the flow field and heat transfer through the specification of the free-surface boundary condition in Eq. (B.28). The normal component of the velocity at the interface is directly proportional to the Jakob number. For high Jakob numbers, differences between the Nusselt number predictions for situations with and without evaporation would be expected. The results shown in Fig. B.4 are for relatively low Jakob numbers, $\mathrm{Ja}<0.03$.

In Fig. B.5, results for both water and methanol at higher Jakob numbers, $J a=0.2$, are shown. The Jakob number is increased by increasing the temperature difference across the liquid layers to $100 \mathrm{~K}$ and reducing the acceleration rates to $0.54 \mathrm{~g}-5.4 \mathrm{~g}$ for water and $0.46 g-4.6 g$ for methanol. Although these parameters represent unphysical 


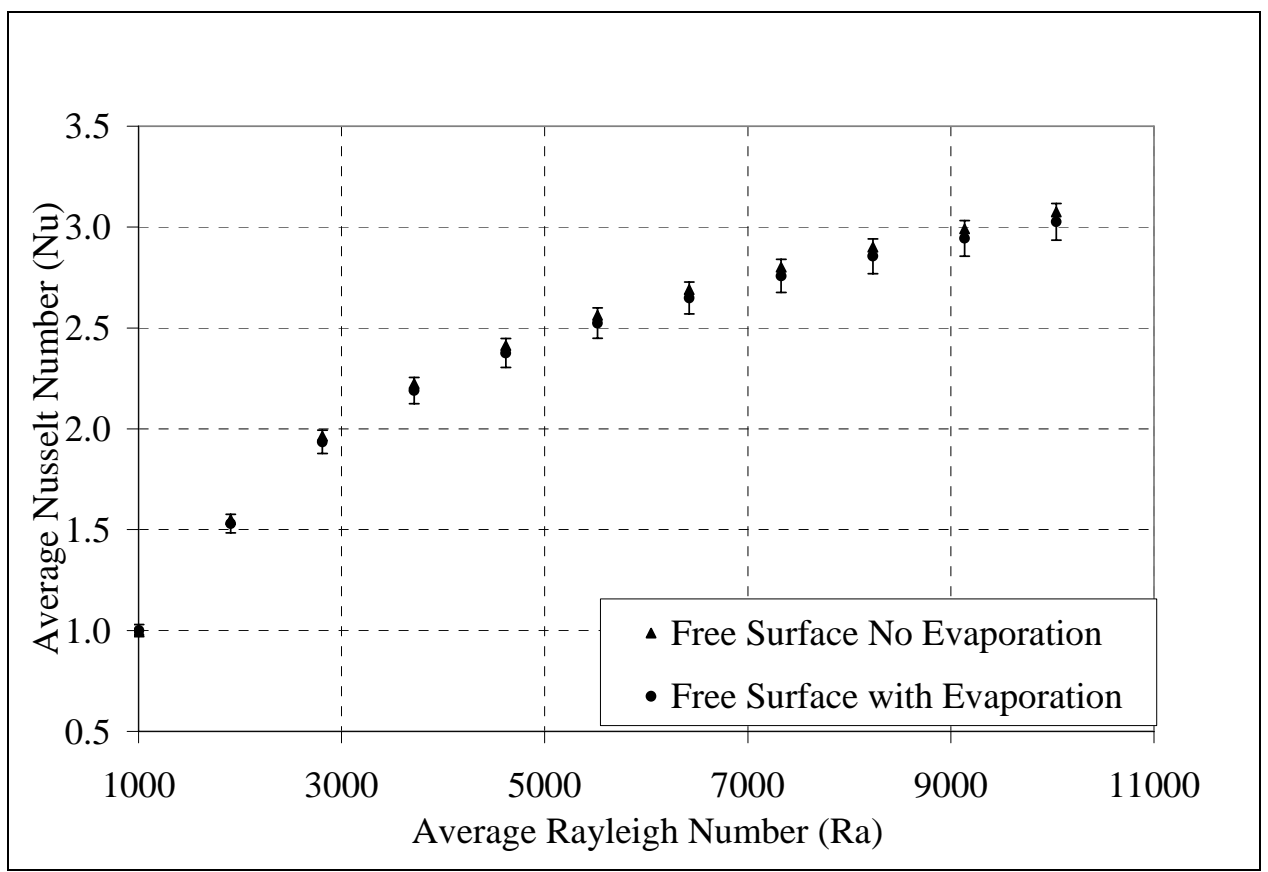

(a)

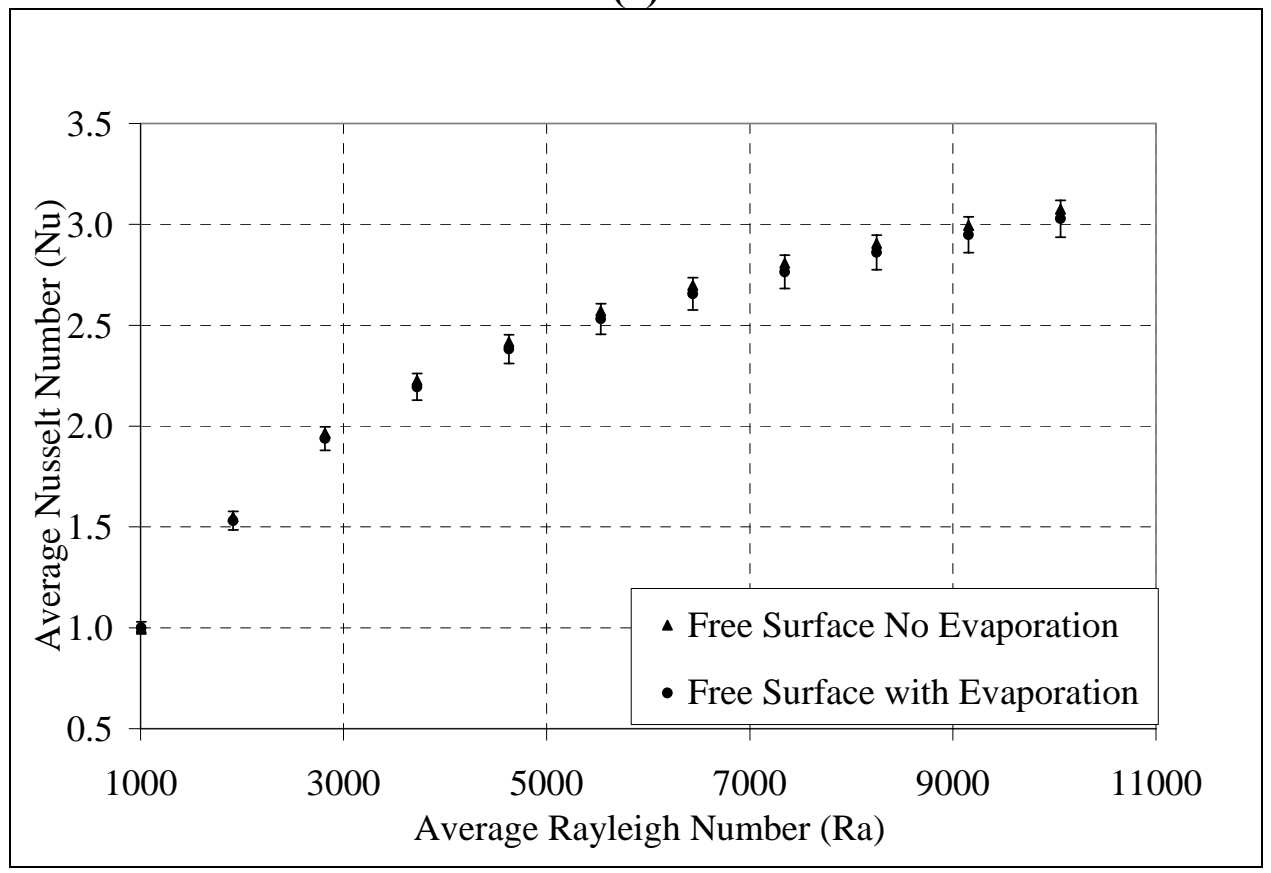

(b)

Figure B.5. Comparing the free-surface case with evaporation to the free-surface case without evaporation with a Jakob number of 0.2 in (a) water and (b) methanol.

cases (it is likely that boiling would be induced for these large temperature differences) the sensitivity of the solutions to Jakob number can be assessed. The figure shows only a 
slight $(<2 \%)$ reduction in Nusselt number for the cases with surface evaporation compared to those without.

In Fig. B.6, the results of all simulations for water and methanol with free-surface evaporation are presented on a single plot. A curve fit, similar to the expression used by Hollands et al. (Hollands et al. 1975) for the Rayleigh-Bénard problem, is applied to the free-surface evaporation data. The correlation

$$
N u=1+2.2(1-1600 / R a)^{*}+\left[(R a / 5830)^{1 / 3}-1\right]^{*},
$$

is a good fit to the numerical data for water and methanol working fluids (the largest error is $16 \%$ at the $R a=1900$ data point; otherwise the correlation fits to within the $3.4 \%$ GCI). The notion [ ] indicates that if a negative quantity is calculated in the bracketed term, the bracketed term is taken as zero. This correlation ignores the influences of surface evaporation (which scales with the Jakob number) and of surface deformation. Both of these effects have been shown to be negligible in the heat pipe application.

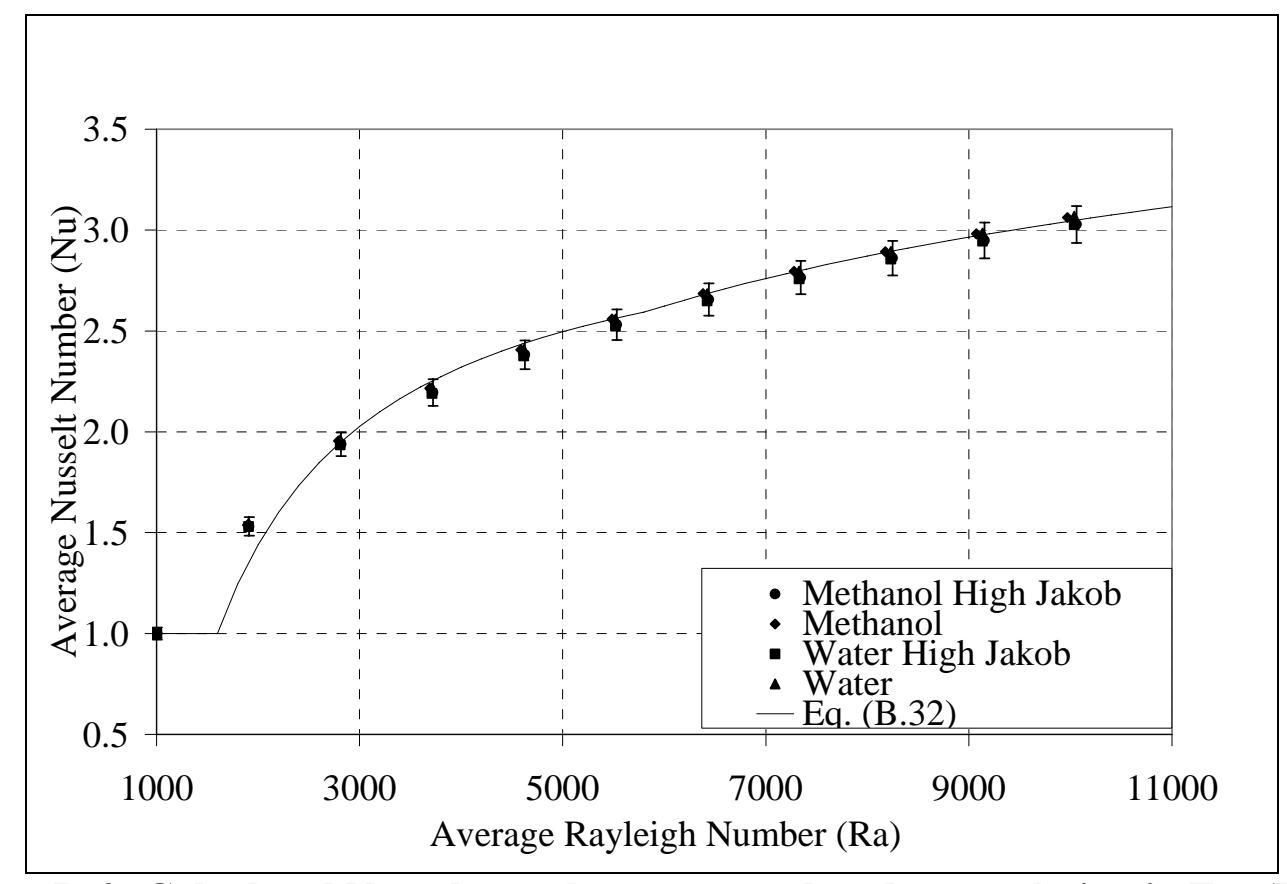

Figure B.6. Calculated Nusselt number compared to the correlation in Eq. (B.32). 


\section{APPENDIX C: Experimental UnCERTAinty Analysis}

The uncertainties in measuring the heat input to the heat pipe, $\varepsilon(Q)$ (see Fig. 5.4), and temperatures, $\varepsilon(T)( \pm 0.5 K)$, will propagate to calculated results, $\Psi$. Assuming that $\Psi=\Psi(T, Q)$, the best representation for the uncertainty $\varepsilon(\Psi)$ is obtained by the Kline-McClintock second power law (Figliola and Beasley 1995)

$$
\varepsilon(\psi)=\sqrt{\left[\frac{\partial \psi}{\partial T} \varepsilon(T)\right]^{2}+\left[\frac{\partial \psi}{\partial Q} \varepsilon(Q)\right]^{2}} .
$$

In Chapter 5, temperature differences $\Delta T=T_{1}-T_{2}$, thermal conductance $G=Q / \Delta T$, ratio of Nusselt numbers $N u / N u_{\min }=G / G_{\min }$, and heat transfer coefficients $h_{e}=G / A_{e}$ are presented. Applying Eq. (C.1) gives

$$
\varepsilon(\Delta T)=\sqrt{\left[\varepsilon\left(T_{1}\right)\right]^{2}+\left[-\varepsilon\left(T_{2}\right)\right]^{2}}=\sqrt{2} \varepsilon(T) .
$$

Similarly, errors in the other calculated variables can be obtained as

$$
\begin{gathered}
\varepsilon(G)=\sqrt{\left[\frac{\varepsilon(Q)}{\Delta T}\right]^{2}+\left[-\frac{G \varepsilon(\Delta T)}{\Delta T}\right]^{2}} \\
\varepsilon\left(\frac{N u}{N u_{\text {min }}}\right)=\sqrt{\left[\frac{\varepsilon(G)}{G_{\min }}\right]^{2}+\left[-\frac{G \varepsilon\left(G_{\min }\right)}{G_{\text {min }}^{2}}\right]^{2}} \\
\varepsilon\left(h_{e}\right)=\varepsilon(G) / A_{e} .
\end{gathered}
$$




\section{REFERENCES}

Bailey, R. L., and Thronton, G. K. (1978). "Oxford Rotating Helium Rig." ICEC 7, London, 386-393.

Baker, J., Oliver, T., Lin, L., Ponnappan, R., and Leland, J. E. (2001). "Correlations of Critical Froude Number for Annular-Rimming Flow in Rotating Heat Pipes." Journal of Fluids Engineering, 123(4), 909-913.

Baker, J., Ponnappan, R., and Leland, J. E. (1999). "A Computational Model of Transport Within a Rotating Heat Pipe." 37th AIAA Aerospace Sciences Meeting and Exhibit, Reno, NV.

Baldor Electric Co., PO Box 2400, Fort Smith AR, 72901-8394, Model \# JMSM3613T-2.

Bejan, A. (1995). "Convection Heat Transfer." John Wiley \& Sons, New York.

Bird, R. B., Stewart, W. E., and Lightfoot, E. N. (2002). Transport Phenomena, John Wiley \& Sons, New York.

Busse, C. A. (1967). "Pressure Drop in the Vapor Phase of Long Heat Pipes." Thermionic Conversion Specialist Conference, Palo Alto, California.

Cao, Y., and Chang, W. S. (1997). "Analyses of Heat Transfer Limitations of Radially Rotating Heat Pipes for Turbomachinery Applications." 32nd AIAA Thermophysics Conference, AIAA-97-2542.

Cao, Y., and Faghri, A. (1991). "Transient Multidimensional Analysis of Nonconventional Heat Pipes with Uniform and Nonuniform Heat Distributions." Journal of Heat Transfer, 113, 995-1002.

Cao, Y., and Faghri, A. (1993). "Conjugate Modeling of High-Temperature Nosecap and Wing Leading Edge Heat Pipes." Journal of Heat Transfer, 115, 819-822.

Castle, R. M., Thomas, S., and Yerkes, K. L. (2000). "The Effect of Working Fluid Inventory on the Performance of Revolving Helically-Grooved Heat Pipes." 34th National Heat Transfer Conference, Pittsburgh, PA.

Castle, R. M., Thomas, S. K., and Yerkes, K. L. (2001). "The Effect of Working Fluid Inventory on the Performance of Revolving Helically Grooved Heat Pipes." Journal of Heat Transfer, 123, 120-129.

Catton, I. (1972). "The Effect of Insulating Vertical Walls on the Onset of Motion in a Fluid Heated From Below." International Journal of Heat and Mass Transfer, 15(4), 665-672. 
Cengel, Y. A. (1998). Heat Transfer: A Practical Approach, McGraw-Hill, Boston.

Chandrasekhar, S. (1961). Hydrodynamic and Hydromagnetic Instability, Oxford University Press.

Chen, C., and Floryan, J. M. (1994). "Numerical Simulation of Nonisothermal Capillary Interfaces." Journal of Computational Physics, 111, 183-193.

Churchill, S. W. (1977). "A Comprehensive Correlating Equation for Laminar, Assisting, Forced and Free Convection." AIChE Journal, 23(1), 10-16.

Cotter, T. P. (1965). "Theory of Heat Pipes." LA-3246-MS, Los Alamos National Laboratory, Los Alamos, New Mexico.

Curtila, R., and Chataing, T. (1984). "Experimental Study of a Revolving Heat Pipe." 5th International Heat Pipe Conference, Tsukuba, Japan.

Cuvelier, C., and Driessen, J. M. (1986). "Thermocapillary Free Boundaries in Crystal Growth." Journal of Fluid Mechanics, 169(1), 1-26.

Daniels, T. C., and Al-Jumaily, F. K. (1975). "Investigations of the Factors Affecting the Performance of a Rotating Heat Pipe." International Journal of Heat and Mass Transfer, 18, 961-973.

de Vahl Davis, G. (1983). "Natural Convection of Air in a Square Cavity: A Bench Mark Numerical Solution." International Journal for Numerical Methods in Fluids, 3(3), 249-264.

Eaton. (2003). "Hose Assembly Master Catalog." W-HYOV-MC002-E, Eaton Weatherhead.

F-Chart Software, EES v.6.728, www.fchart.com.

Faghri, A. (1995). Heat Pipe Science and Technology, Taylor \& Francis, Washington, DC.

Faghri, A., Buchko, M., and Cao, Y. (1991a). "A Study of High-Temperature Heat Pipes with Multiple Heat Sources and Sinks: Part I-Experimental Methodology and Frozen Startup Profiles." Journal of Heat Transfer, 113, 1003-1009.

Faghri, A., Buchko, M., and Cao, Y. (1991b). "A Study of High-Temperature Heat Pipes with Multiple Heat Sources and Sinks: Part II-Analysis of Continuum Transient and Steady-State Experimental Data with Numerical Predictions." Journal of Heat Transfer, 113, 1010-1016. 
Faghri, A., Gogineni, S., and Thomas, S. (1993). "Vapor Flow Analysis of an Axially Rotating Heat Pipe." International Journal of Heat and Mass Transfer, 36(9), 22932303.

Faghri, M., Sparrow, E. M., and Prata, A. T. (1984). "Finite-Difference Solutions of Convection-Diffusion Problems in Irregular Domains, Using a Nonorthogonal Coordinate Transformation." Numerical Heat Transfer, 7(2), 183-209.

Figliola, R. S., and Beasley, D. E. (1995). Theory and Design for Mechanical Measurements, John Wiley \& Sons Inc., New York.

Fox, R. W., and McDonald, A. T. (1992). Introduction to Fluid Mechanics, John Wiley and Sons, New York.

Gi, K., and Maezawa, S. (1990). "Heat Transfer Characteristics of a Parallel Rotating Heat Pipe." 7th International Heat Pipe Conference, Minsk, USSR.

Giessler, F., Sattler, P. K., and Thoren, F. (1987). "Heat Pipe Cooling of Electrical Machines." 6th International Heat Pipe Conference, Grenoble, France.

Gray, V. (1969). "The Rotating Heat Pipe-A Wickless Hollow Shaft for Transferring High Heat Fluxes." ASME/AIChE Heat Transfer Conference, 69-HT-19, Minneapolis, 1-5.

Greenspon, J. E. (1976). "An Approximate Nondimensional Representation of the Thor Equations." 178, US Army Material Systems Analysis Activity, Aberdeen Proving Ground, MD.

Grover, G. M., Cotter, T. P., and Erickson, G. F. (1964). "Structures of Very High Thermal Conductance." Journal of Applied Physics, 35(6), 1990-1991.

Harley, C., and Faghri, A. (1995). "Two-Dimensional Rotating Heat Pipe Analysis." Journal of Heat Transfer, 117, 202-208.

Hieber, C. A. (1982). "Laminar Mixed Convection in an Isothermal Horizontal Tube: Correlation of Heat Transfer Data." International Journal of Heat and Mass Transfer, 25(11), 1737-1746.

Hill, D. D. (2003). G-10 Structural Analysis, E-mail to T. Jankowski.

Hollands, K. G. T. (1983). "Multi-Prandtl Number Correlation Equations for Natural Convection in Layers and Enclosures." International Journal of Heat and Mass Transfer, 27(3), 466-468. 
Hollands, K. G. T., Raithby, G. D., and Konicek, L. (1975). "Correlation Equations for Free Convection Heat Transfer in Horizontal Layers of Air and Water." International Journal of Heat and Mass Transfer, 18(7-8), 879-884.

Ismail, K. A., and Miranda, R. F. (1997). "Two-Dimensional Axisymmetrical Model for a Rotating Porous Wicked Heat Pipe." Applied Thermal Engineering, 17(2), 135-155.

Issacci, F., Catton, I., and Ghoniem, N. M. (1991). "Vapor Dynamics of Heat Pipe StartUp." Journal of Heat Transfer, 113, 985-994.

Jang, J. H., Faghri, A., and Chang, W. S. (1991). "Analysis of the One-Dimensional Transient Compressible Vapor Flow in Heat Pipes." International Journal of Heat and Mass Transfer, 34(8), 2029-2037.

Jankowski, T. A., Razani, A., Prenger, F. C., and Schmierer, E. N. (2006a). "Natural Convection in the Evaporator Sections of Rotating Heat Pipes." International Journal of Heat and Mass Transfer, submitted.

Jankowski, T. A., Razani, A., Schmierer, E. N., Stewart, J. A., Hill, D. D., and Prenger, F. C. (2006b). "Apparatus for Testing Rotating Heat Pipes." 9th AIAA/ASME Joint Thermophysics and Heat Transfer Conference, San Francisco, CA.

Jankowski, T. A., Waynert, J. A., Prenger, F. C., and Razani, A. (2004). "Steady State Modeling of a Rotating Heat Pipe with a Composite Wick Structure." ASME Heat Transfer/Fluids Engineering Summer Conference, HT-FED2004-56083, Charlotte NC.

Jen, T. C., Gutierrez, G., Eapen, S., Barber, G., Zhao, H., Szuba, P. S., Labataille, J., and Manjunathaiah, J. (2002). "Investigation of Heat Pipe Cooling in Drilling Applications. Part I: Preliminary Numerical Analysis and Verification." International Journal of Machine Tools and Manufacture, 42(5), 643-652.

Jian, C., Chuanjing, T., and Zhongyan, Z. (1987). "Condenser Heat Transfer in Inclined Rotating Heat Pipe." 6th International Heat Pipe Conference, Grenoble, France, 535538.

Jian, C., Lou, D. Y. S., and Chuanjing, T. (1990). "Investigation of the Evaporation Heat Transfer in the Rotating Heat Pipes." 7th International Heat Pipe Conference, Minsk, USSR, 349-356.

Judd, R. L., Aftab, K., and Elbestawi, M. A. (1994). "An Investigation of the Use of Heat Pipes for Machine Tool Spindle Bearing Cooling." International Journal of Machine Tools and Manufacture, 34(7), 1031-1043. 
Katsuta, M., Kigami, N., Nagata, K., Sotani, J., and Koizumi, T. (1984). "Performance and Characteristics of a Rotating Heat Pipe." 5th International Heat Pipe Conference, Tsukuba, Japan, 106-112.

Khalkhali, H., Faghri, A., and Zuo, Z. J. (1999). "Entropy Generation in a Heat Pipe System." Applied Thermal Engineering, 19, 1027-1043.

Khmelev, Y. A., and Shevel, E. V. (1991). "Theoretical Analysis of Condensation in a Rotating Heat Pipe at Low Angular Velocity of Rotation." Journal of Engineering Physics and Thermophysics, 60(1), 14-18.

King, M. P., and Wilson, M. (2005). "Numerical Simulations of Convective Heat Transfer in Rayleigh-Benard Convection and a Rotating Annulus." Numerical Heat Transfer, Part A, 48(6), 529-545.

Klasing, K. S., Thomas, S. K., and Yerkes, K. L. (1999). "Prediction of the Operating Limits of Revolving Helically Grooved Heat Pipes." Journal of Heat Transfer, 121(1), 213-217.

Körner, W. (1970). "Effect of High Acceleration on Heat Transfer During Boiling." Chemie Ingenieur Technik, 42(6), 409.

Krivosheev, B. N., Kukharskii, M. P., and Portnov, V. D. (1979). "Heat Transfer in the Evaporator Section of a Rotating Heat Pipe at Low Rotational Speeds." Journal of Engineering Physics and Thermophysics, 37(1), 773-778.

Langston, L., and Faghri, A. (1995). "Heat Pipe Turbine Blade Cooling." Advanced Turbine Systems Annual Program Review, Morgantown, West Virginia.

Lee, J. S., and Kim, C. J. (2001). "Heat Transfer and Internal Flow Characteristics of a Coil-Inserted Rotating Heat Pipe." International Journal of Heat and Mass Transfer, 44, 3543-3551.

Leonard, B. P. (1979). "A Stable and Accurate Convective Modelling Procedure Based on Quadratic Upstream Interpolation." Computer Methods in Applied Mechanics and Engineering, 19(1), 59-98.

Li, H. M., Liu, C. Y., and Domodaran, M. (1993). "Analytical Study of the Flow and Heat Transfer in a Rotating Heat Pipe." Heat Recovery Systems and CHP, 13(2), 115122.

Lin, L., and Faghri, A. (1999). "Heat Transfer in Micro Region of a Rotating Miniature Heat Pipe." International Journal of Heat and Mass Transfer, 42, 1363-1369. 
Lin, L., and Groll, M. (1996). "Critical Conditions for Collapse of Annular Flow in a Rotating Heat Pipe with a Cylindrical Wall." Heat Transfer Engineering, 17(3), 2934.

Ling, J., and Cao, Y. (2000). "Closed-Form Analytical Solutions for Radially Rotating Miniature High-Temperature Heat Pipes Including Non-Condensable Gas Effects." International Journal of Heat and Mass Transfer, 43, 3661-3671.

Ling, J., Cao, Y., and Lopez, A. P. (2001). "Experimental Investigations of Radially Rotating Miniature High-Temperature Heat Pipes." Journal of Heat Transfer, 123, 113-119.

Machado, H. A., and Miranda, R. F. (2003). "Operation Limits for Rotating Cylindrical Heat Pipes." Numerical Heat Transfer, 44, 299-313.

Maezawa, S., Takuma, M., and Tsuchida, A. (1984). "Application of Disk-Shaped Rotating Heat Pipe to Brake Cooling." Heat and Mass Transfer in Rotating Machinery, Hemisphere, Washington D. C., 659-668.

Marto, P. J. (1976). "Performance Characteristics of Rotating, Wickless Heat Pipes." 2nd International Heat Pipe Conference, Bologna, Italy, 281-291.

Marto, P. J. (1984). "Rotating Heat Pipes." Heat and Mass Transfer in Rotating Machinery, Hemisphere, Washington D. C., 609-632.

Marto, P. J., and Gray, V. H. (1971). "Effects of High Accelerations and Heat Fluxes on Nucleate Boiling of Water in an Axisymmetric Rotating Boiler." NASA TN D-6307, NASA Lewis Research Center, Cleveland, $\mathrm{OH}$.

Nagendra, H. R. (1973). "Interaction of Free and Force Convection in Horizontal Tubes in the Transition Regime." Journal of Fluid Mechanics, 57(2), 269-288.

Nakayama, W., Ohtsuka, Y., Itoh, H., and Yoshikawa, T. (1984). "Optimum Charge of Working Fluids in Horizontal Rotating Heat Pipes." Heat and Mass Transfer in Rotating Machinery, Hemisphere, Washington D. C., 633-644.

Nerowski, G., Frauenhofer, J., Ries, G., Nick, W., and Neumuller, H. W. (2004). "Advances and Prospects of HTS Rotating Machine Development at Siemens." IEEE Power Engineering Society General Meeting, 1 and 2, 2052-2055.

Niekawa, J., Matsumoto, K., Koizumi, T., Hasegawa, K., Kaneko, H., and Mizoguchi, Y. (1981). "Performance of Revolving Heat Pipes and Application to a Rotary Heat Exchanger." 4th International Heat Pipe Conference, London, UK.

Omega Engineering Inc., One Omega Drive, PO Box 4047, Stamford CT, 06907-0047. 
Osborne, D. G., and Incropera, F. P. (1985a). "Experimental Study of Mixed Convection Heat Transfer for Transitional and Turbulent Flow Between Horizontal, Parallel Plates." International Journal of Heat and Mass Transfer, 28(7), 1337-1344.

Osborne, D. G., and Incropera, F. P. (1985b). "Laminar, Mixed Convection Heat Transfer for Flow Between Horizontal Parallel Plates with Asymmetric Heating." International Journal of Heat and Mass Transfer, 28(1), 207-217.

Oslejsek, O., and Polasek, F. (1976). "Cooling of Electrical Machines by Heat Pipes." 2nd International Heat Pipe Conference, Bologna, Italy, 503-514.

Pacheco, J. R., and Peck, R. E. (2000). "Nonstaggered Boundary-Fitted Coordinate Method for Free Surface Flows." Numerical Heat Transfer, Part B, 37, 267-291.

Patankar, S. V. (1980). Numerical Heat Transfer and Fluid Flow, Hemisphere Publishing, Washington D. C.

Patankar, S. V., and Spalding, D. B. (1972). "A Calculation Procedure for Heat, Mass and Momentum Transfer in Three-Dimensional Parabolic Flows." International Journal of Heat and Mass Transfer, 15, 1787-1806.

Peterson, G. P., and Wu, D. (1993). "A Review of Rotating and Revolving Heat Pipes." Heat and Technology, 11(1-2), 191-218.

Pokorny, B., Polasek, F., Schneller, J., and Stulc, P. (1984). "Heat Transfer in Co-Axial and Parallel Rotating Heat Pipes." 5th International Heat Pipe Conference, Tsukuba, Japan.

Ponnappan, R., and He, Q. (1998). "Test Results of Water and Methanol High Speed Rotating Heat Pipes." Journal of Thermophysics and Heat Transfer, 12(3), 391-297.

Ponnappan, R., and Leland, J. E. (1998). "Rotating Heat Pipe for High Speed Motor/Generator Cooling." Aerospace Power Systems Conference, Williamsburg, Virginia.

Prantap, V. S., and Spalding, D. B. (1976). "Fluid Flow and Heat Transfer in Threedimensional Duct Flows." International Journal of Heat and Mass Transfer, 19, 1183-1188.

Prenger, F. C. (1983). "Performance Limits of Gravity-Assisted Heat Pipes." 5th International Heat Pipe Conference, Tsukuba, Japan.

Prenger, F. C. (2003). "Rotating Heat Pipe Assembly Penetration Hazard Analysis." ESAAET:04-09, Los Alamos National Laboratory, Los Alamos NM. 
Reddy, R. S., Venkateswarlu, P., and Sastri, V. M. K. (1987). "Experimental Heat Transfer Characteristics of a Rotating Heat Pipe with an Air-Cooled Condenser." 6th International Heat Pipe Conference, Grenoble, France.

Reddy, R. S., Venkateswarlu, P., and Sastri, V. M. K. (1990). "Effect of Inclination on the Performance of Rotating Heat Pipe." 7th International Heat Pipe Conference, Minsk, USSR.

Richter, R., and Gottschlich, J. M. (1994). "Thermodynamic Aspects of Heat Pipe Operation." Journal of Thermophysics and Heat Transfer, 8(2), 334-340.

Roache, P. J. (1998). Verification and Validation in Computational Science and Engineering, Hermosa, Albuquerque, NM.

Roache, P. J. (2003). "Conservatism of the Grid Convergence Index in Finite Volume Computations on Steady-State Fluid Flow and Heat Transfer." Journal of Fluids Engineering, 125(4), 731-732.

Salinas, D., and Marto, P. J. (1991). "Analysis of an Internally Finned Rotating Heat Pipe." Numerical Heat Transfer, 19, 255-275.

Semena, M. G., Khmelev, Y. A., and Shevel, E. V. (1991). "Distribution of Heat Transfer Agent in the Capillary Structure of Rotating Heat Pipes with a Displaced Axis of Rotation." Journal of Engineering Physics, 60(5), 642-645.

Shimizu, A., and Yamazaki, S. (1987). "Helical Guide-Type Rotating Heat Pipes." 6th International Heat Pipe Conference, Grenoble, France.

Sim, B. C., Kim, W. S., and Zebib, A. (2004). "Axisymmetric Thermocapillary Convection in Open Cylindrical Annuli with Deforming Interfaces." International Journal of Heat and Mass Transfer, 47, 5365-5373.

Slattery, J. C. (1999). Advanced Transport Phenomena, Cambridge University Press, New York.

Song, F., Ewing, D., and Ching, C. Y. (2003). "Fluid Flow and Heat Transfer Model for High-Speed Rotating Heat Pipes." International Journal of Heat and Mass Transfer, 46, 4393-4401.

Song, F., Ewing, D., and Ching, C. Y. (2004). "Experimental Investigation on the Heat Transfer Characteristics of Axial Rotating Heat Pipes." International Journal of Heat and Mass Transfer, 47, 4721-4731.

Streby, M. A., Ponnappan, R., Leland, J. E., and Beam, J. E. (1996). "Design and Testing of a High Speed Rotating Heat Pipe." IECEC 96, Washington, D. C., 1453-1458. 
Takahashi, Y., Umeda, H., Sumomogi, T., Hashimoto, R., and Mizuta, K. (1995a). "Flow Pattern of Working Fluid and Heat Transfer Performance in Annular Type Rotating Heat Pipes with Horizontal Axis I: Flow Pattern and the Heat Transport Rate at the Evaporation Section." Heat Transfer-Japanese Research, 24(5), 409-424.

Takahashi, Y., Umeda, H., Sumomogi, T., Hashimoto, R., and Mizuta, K. (1995b). "Heat Transfer Rate of Annular-Type Horizontal Rotating Heat Pipes II. Experiments and Theoretical Analysis for the Heat Transport Rate." Heat Transfer-Japanese Research, 24(8), 672-688.

Thoren, F. (1984). "Heat Pipe Cooled Induction Motors." 5th International Heat Pipe Conference, Tsukuba, Japan.

Tien, C. L., and Chung, K. S. (1979). "Entrainment Limits in Heat Pipes." AIAA Journal, 17(6), 643-646.

Tournier, J. M., and El-Genk, M. S. (1994). "A Heat Pipe Transient Analysis Model." International Journal of Heat and Mass Transfer, 37(5), 753-762.

Tournier, J. M., and El-Genk, M. S. (1996). "A Vapor Flow Model for Analysis of Liquid-Metal Heat Pipe Startup from a Frozen State." International Journal of Heat and Mass Transfer, 39(18), 3767-3780.

Tournier, J. M., and El-Genk, M. S. (2003). "Startup of a Horizontal LithiumMolybdenum Heat Pipe from a Frozen State." International Journal of Heat and Mass Transfer, 46, 671-685.

Unique Wire Weaving Co., Inc., 762 Ramsey Ave., Hillside, N.J. 07205, SS Type 316 Plain Weave.

Urbahn, J. A., Ackermann, R. A., Huang, X., Laskaris, E. T., Sivasubramaniam, K., and Steinbach, A. (2004). "The Thermal Performance of a 1.5 MVA HTS Generator." Advances in Cryogenic Engineering: Transactions of the Cryogenic Engineering Conference, 49, 849-858.

Vasiliev, L. L., and Khrolenok, V. V. (1976). "Centrifugal Coaxial Heat Pipes." 2nd International Heat Pipe Conference, Bologna, Italy, 293-302.

Waowaew, N., Terdtoon, P., Maezawa, S., Kamonpet, P., and Klongpanich, W. (2003). "Correlation to Predict Heat Transfer Characteristics of a Radially Rotating Heat Pipe at Vertical Position." Applied Thermal Engineering, 23, 1019-1032.

Woloshun, K. A., Merrigan, M. A., and Best, E. D. (1988). "HTPIPE: A Steady-State Heat Pipe Analysis Program." LA-11324-M, Los Alamos National Laboratory, Los Alamos, New Mexico. 
Zuo, Z. J., and Faghri, A. (1997). "A Network Thermodynamic Analysis of the Heat Pipe." International Journal of Heat and Mass Transfer, 41(11), 1473-1484. 
This report has been reproduced directly from the best available copy. It is available electronically on the Web (http://www.doe.gov/bridge).

Copies are available for sale to U.S. Department of Energy employees and contractors from:

Office of Scientific and Technical Information

P.O. Box 62

Oak Ridge, TN 37831

(865) 576-8401

Copies are available for sale to the public from: National Technical Information Service

U.S. Department of Commerce

5285 Port Royal Road

Springfield, VA 22161

(800) 553-6847 
- Los Alamos NATIONAL LABORATORY

EST.1943 